
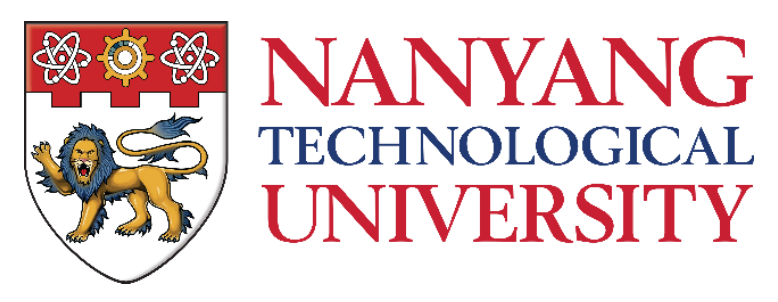

Silicon Heterojunction Solar Cell Based on Organic and Transition Metal Oxide Selective Carrier Contact Layer

\author{
Ari Bimo Prakoso \\ G1203383A
}

\title{
SCHOOL OF ELECTRICAL AND ELECTRONIC ENGINEERING
}

A thesis submitted to the Nanyang Technological University in partial fullfillment of the requirement for the degree of Doctor of Philosophy 


\section{Acknowledgements}

I would like to take this opportunity to express my deepest appreciation and gratitude to my supervisor Assoc. Prof. Rusli, for giving me an opportunity to pursue a higher degree and an interesting research project. He has provided continuous support and valuable guidance throughout my research period even when he has a tight schedule. His scientific and ethical standards inspire me to develop myself to be a better researcher. In the hard time, he would always encourage me to persevere and to overcome difficulties.

In addition, I would like to thank Institute of Materials Research and Engineering (IMRE), Singapore for the opportunity of research student attachment program. I would like to express my gratitude to Dr. Jiang Changyun, my co-supervisor from IMRE, for his guidance and knowledge in organic semiconductors topic. I am also grateful to Dr. Ke Lin for her help with the hybrid device characterization.

Furthermore, I would also like to thanks the support provided by our Clean Room and Characterization Laboratory technicians, specifically Mr. M. Shamsul, Mr. Mak Foo Wah, Ms. Seet Lye Ping, Ms. Ngo Ling Ling, Ms. Yang Xiaohong and Mr. M. Fauzi. I would also want to thank our group post-doc Dr. Wang Jianxiong, and my group mates Dr. Hong Lei, Dr. Alienor Togonal, Dr. Wang Hao, Mr. Li Zeyu and Mr. Lu Chenjin for their kind supports and discussions in the course of my research project. 
Last but not least, I would like to give my special appreciation to my family and all my friends, from whom I received a lot of love, patience, understanding, and encouragement through my studies. 


\section{Abstract}

Si heterojunction solar cells based on organic and transition metal oxide selective carrier contact (SCC) layer are developed to bypass the high temperature diffusion process required for the fabrication of $\mathrm{p}$-n junction in conventional Si solar cell. In this work, we design, simulate, fabricate and characterize Si heterojunction solar cells based on organic material, poly(3,4-ethylenedioxythiophene) : polystyrenesulphonate (PEDOT:PSS), and transition metal oxide, molybdenum oxide $\left(\mathrm{MoO}_{\mathrm{x}}\right)$.

To have an in-depth understanding of the n-Si/PEDOT:PSS hybrid solar cell, we characterize the heterojunction by reverse recovery transient (RRT) and DC I-V techniques to ascertain its nature. Solar cells are fabricated by spin coating PEDOT:PSS solution on top of $\mathrm{n}-\mathrm{Si}$ with various doping concentrations $\left(N_{\mathrm{D}}\right)$ from $10^{14}-10^{17} \mathrm{~cm}^{-3}$. The results of RRT measurement contradicts the Schottky junction model that is commonly assumed for such heterojunction. Instead the nSi/PEDOT:PSS junction act consistently with the $n-p^{+}$junction model. The forward bias current injection level is found to influence $\tau_{p}$, attributed to the trap saturation effect at $n-S i$ and PEDOT:PSS interface. The interpretation of $n-p^{+}$junction model is also consistent with DC-IV measurement. Photovoltaic measurement of the nSi/PEDOT:PSS hybrid solar cell has been conducted to understand the effect of $N_{D}$ on solar cell performance. 
Having established the nature of the n-Si/PEDOT:PSS junction, we simulate the performance of the hybrid solar cell with different device architectures, using a device simulation software. Four device architectures are studied, which include the conventional front junction (FJ) cell, back junction (BJ) cell, interdigitated back contact (IBC) cell and FJ structure with ARC compensated layer (FJ-ARC) cell. We found that BJ cell in which the PEDOT:PSS layer is located on the back surface of an $\mathrm{n}$-Si substrate can yield PCE of $18.8 \%$, which is higher compared to those of conventional FJ cell with PCE of $15.4 \%$. This is attributed to the elimination of parasitic absorption in PEDOT:PSS in BJ cell. Our simulation also showed that the the parasitic absorption issue affecting the FJ cell architecture could be resolved without resorting to a more demanding BJ structure. We propose to adopt the simpler FJ structure, but reduce the thickness of PEDOT:PSS to $\sim 30 \mathrm{~nm}$, and introduce a layer of silicon nitride anti-reflection coating layer with the thickness of $\sim 40 \mathrm{~nm}$. We obtained a PCE of $18.4 \%$ for the proposed cell structure, which is almost similar to those achieved for the BJ cell. We also simulated the IBC cell in which both the hole and electron collecting contacts are located on the back side of Si. The use of IBC cell structure yields the highest PCE of $20.4 \%$ but require more complicated patterning.

In this work, the use of transition metal oxide, namely molybdenum oxide $\left(\mathrm{MoO}_{\mathrm{x}}\right)$, as the SCC material on the heterojunction solar cells is investigated experimentally. Planar $\mathrm{n}-\mathrm{Si} / \mathrm{MoO}_{x}$ solar cell is fabricated by e-beam evaporation of $\mathrm{MoO}_{x}$ source onto an n-Si substrate to form an electron blocking layer. We found that the PCE is 
almost independent of Si substrate doping $\left(N_{\mathrm{D}}\right)$ at 10.1 to $10.2 \%$ for $N_{\mathrm{D}}=10^{14}$ $10^{16} / \mathrm{cm}^{3}$, which then drops to $6.9 \%$ at higher $N_{D}=10^{17} / \mathrm{cm}^{3}$. It is found that using an evaporated $5 \mathrm{~nm}$ thin ITO layer on top of $\mathrm{MoO}_{x}$ as a barrier layer, a solar cell with PEDOT:PSS electrode can achieve $V_{\text {oc }}=0.592 \mathrm{~V}, \mathrm{FF}=67.3 \%$ and $\mathrm{PCE}=$ 11.3\%. Subsequently, we studied $\mathrm{n}-\mathrm{Si} / \mathrm{MoO}_{\mathrm{x}}$ solar cell with sputtered ITO electrode with or without a barrier layer. We found that the highest PCE $=11.6 \%$ is observed for the solar cell without the barrier layer, yet the $V_{o c}$ is relatively low at $0.554 \mathrm{~V}$. This is in contrast to a solar cell with a barrier layer which yields high $V_{o c}=0.591 \mathrm{~V}$ but has lower PCE $=10.7 \%$.

To develop a solar cell that offers a huge active surface area yet occupies a small footprint, PV-Tower solar cell concept is proposed and studied. The height of PVTower can be increased to boost the power output while its multiple surfaces allow effective collection of diffuse light. We investigated the performance of PV-Tower with an $n^{+}-p-p^{+}$structure using device simulation. Then we calculated the ohmic loss associated with the electrodes on the sidewall of the PV-Tower solar cell, and it is found to scale as a function of the cubic height of the tower. Finally, we also demonstrated with device simulation that PV-Tower cell can also adopt $\mathrm{Si}$ heterojunction solar cell structure with promising result. Therefore, the results suggest that the novel PV-Tower solar cell proposed has a potential to deliver high power output over a small footprint, and is ideal for energy harnessing over a limited physical area, such as in loT applications. 


\section{Table of Contents}

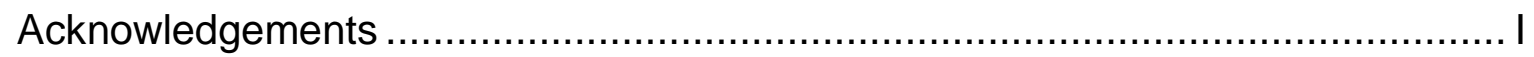

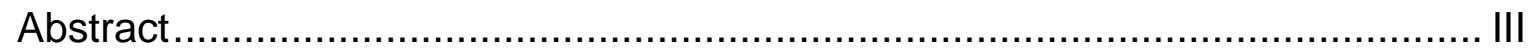

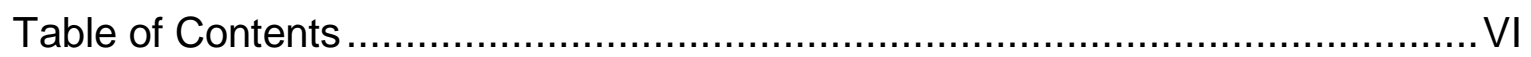

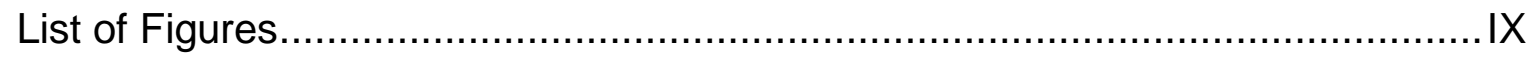

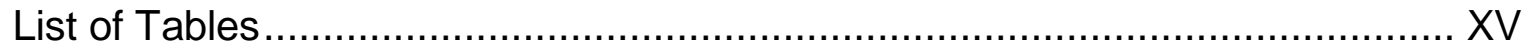

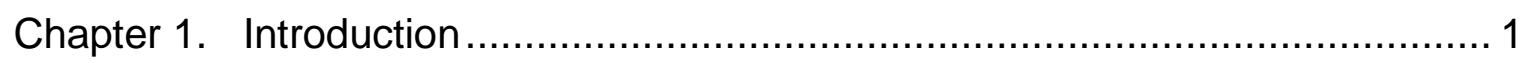

1.1 Background and motivation ...................................................... 1

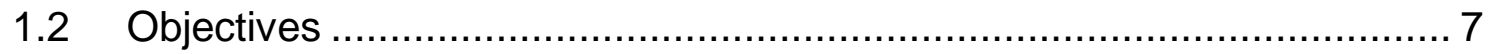

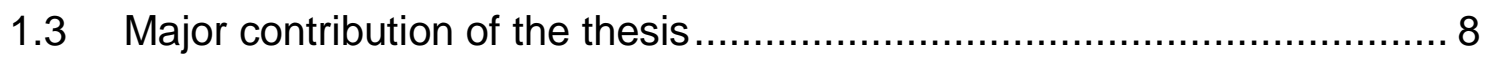

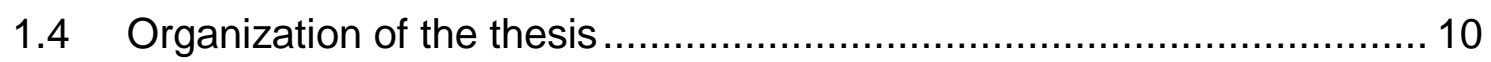

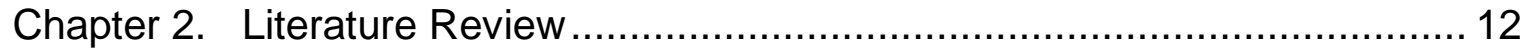

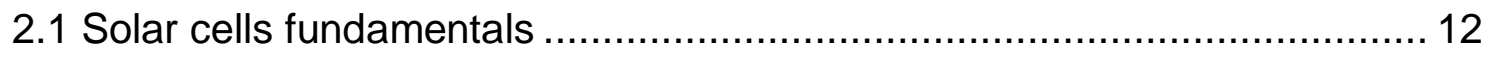

2.1.1 Introduction of solar cell operation ................................................. 12

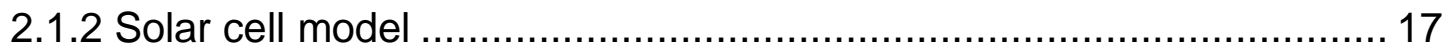

2.2 Silicon, organic and metal oxide based solar cells ................................ 21

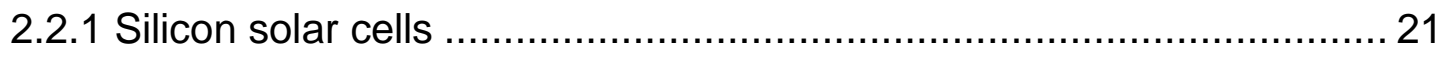

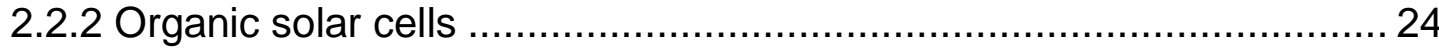

2.2.3 Metal oxide Solar Cells ............................................................ 25

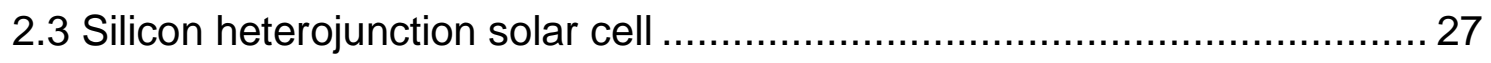

2.3.1 Silicon / organic heterojunction solar cell ...................................... 29

2.3.2 Silicon / transition metal oxide heterojunction solar cell ...................... 38

2.4 Solar cell for small footprint application .............................................. 41

Chapter 3. Fabrication and characterization of planar n-Si/PEDOT:PSS hybrid

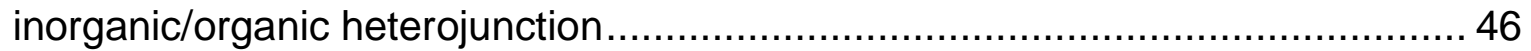

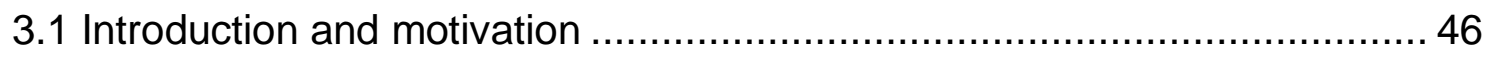

3.2 Fabrication and characterization of planar n-Si/PEDOT:PSS hybrid

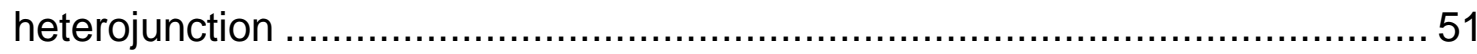

3.2.1 Fabrication of planar n-Si/PEDOT:PSS hybrid ................................. 51

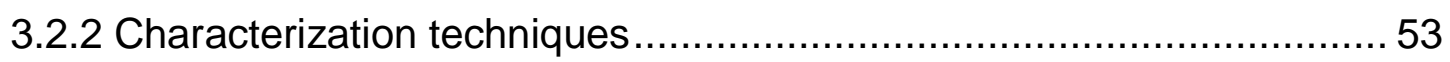

3.3 Study of planar n-Si/PEDOT:PSS hybrid junction behavior ......................57 
3.3.1 Reverse recovery transient characteristic ...................................... 57

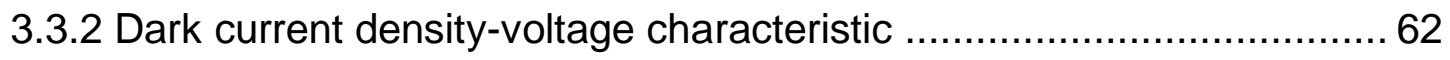

3.3.3 Illuminated current density-voltage characteristic .............................. 68

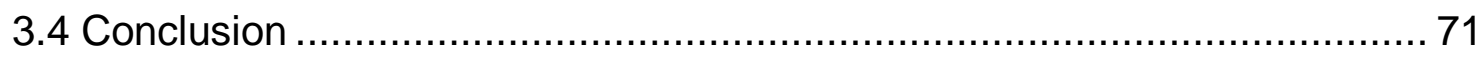

Chapter 4. Simulation and optimization of n-Si/PEDOT:PSS hybrid solar cell with

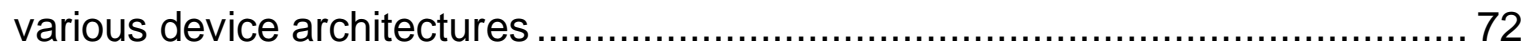

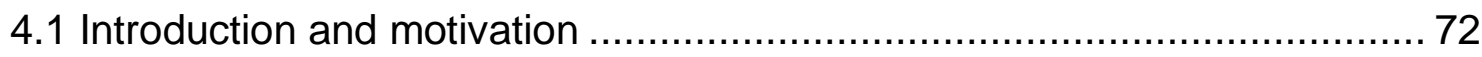

4.2 Simulation of n-Si/PEDOT:PSS hybrid solar cell ..................................... 75

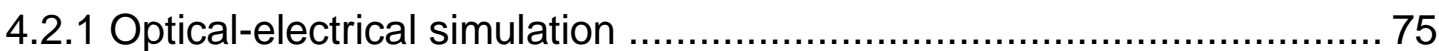

4.2.2 PEDOT:PSS and n-Si electronic properties .................................. 76

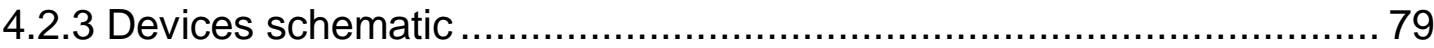

4.3 Conventional and ARC compensated front junction hybrid solar cell ......... 86

4.3.1 Conventional front junction hybrid solar cell ..................................... 86

4.3.2 Front junction hybrid solar cell with ARC compensated layer .............. 90

4.4 Back junction and interdigitated back contact hybrid solar cell ................. 93

4.4.1 Back junction hybrid solar cell ........................................................... 93

4.4.2 Interdigitated back contact hybrid solar cell .................................... 97

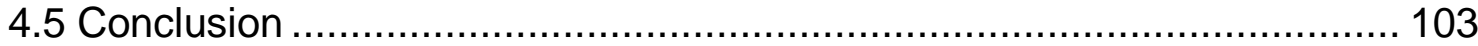

Chapter 5. Fabrication and optimization of Si/molybdenum oxide heterojunction solar cell 105

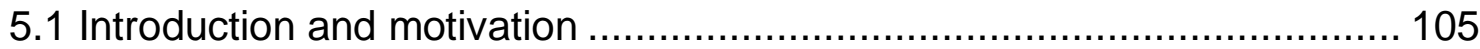

$5.2 \mathrm{Si} /$ molybdenum oxide heterojunction solar cell .................................... 109

5.3 Fabrication and optimization of Si/MoOx heterojunction solar cell .......... 113

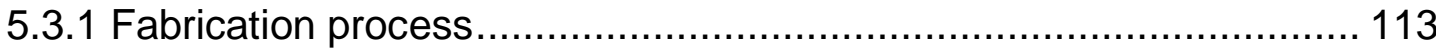

5.3.2 Si/MoOx solar cell with various Si substrate doping ........................ 115

5.3.3 Si/MoOx solar cell with PEDOT:PSS electrode .............................. 118

5.3.4 Si/MoOx solar cell with ITO electrode .......................................... 123

5.3.5 Influence of interfacial Si native oxide on Si/MoOx solar cell ............. 129

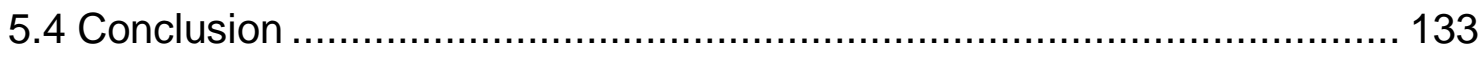

Chapter 6. Simulation of PV-Tower solar cell for high power over small footprint application 134 


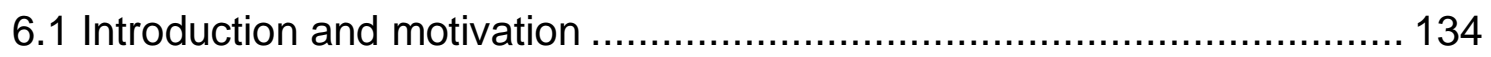

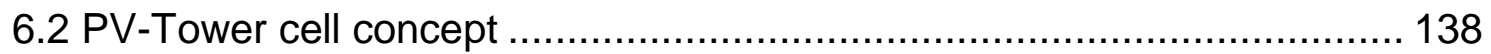

6.2.1. Merit of PV-Tower compared to conventional solar cell................... 138

6.2.2. Industrially Viable Fabrication Process for PV-Tower ....................... 143

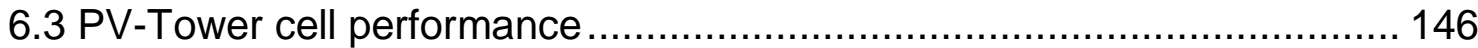

6.3.1 Active surface area per footprint ............................................... 146

6.3.2 Si-based PV-Tower cell response with light incident from different

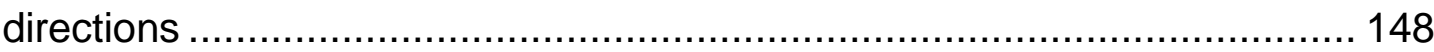

6.3.3. Effect of series resistance on the scaling of PV-Tower solar cell ...... 154

6.4 PV-Tower with Si Heterojunction Solar Cell......................................... 159

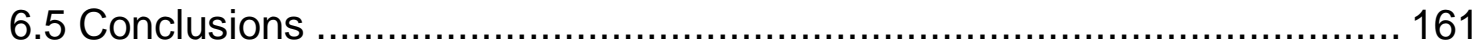

Chapter 7. Conclusion and Future Work .................................................. 163

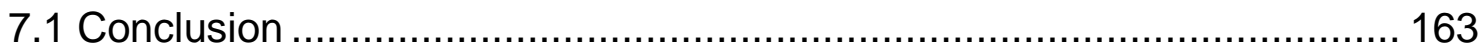

7.2 Recommendations for future research ……....................................... 169

7.2.1 Fabrication of Si/PEDOT:PSS hybrid solar cell with FJ-ARC structure 169

7.2.2 Fabrication of Si/PEDOT:PSS hybrid solar cell with IBC structure .... 169 7.2.3 Study of $\mathrm{n}$-Si/molybdenum oxide solar cell junction behaviour .......... 170 7.2.4 Si/transition metal oxide solar cell with other materials and deposition

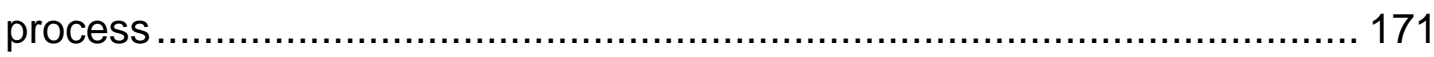

7.2.5 Si/molybdenum oxide solar cell with antireflection nanostructure ...... 171

7.2.6 Fabrication and application of PV-Tower cell ................................. 172

7.2.7 Investigation of ambient temperature effect on photovoltaic parameters 172

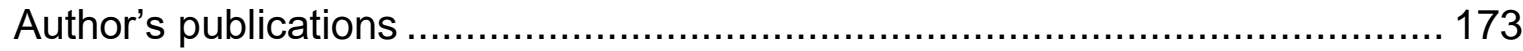

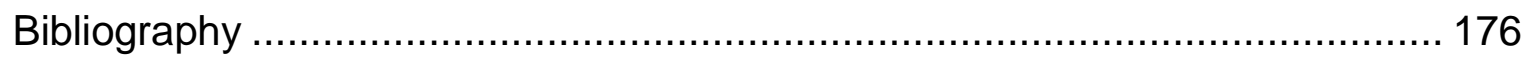




\section{List of Figures}

Figure 1.1: World total primary energy supply from 1971 to 2014 in a unit of Mtoe/year [1]. 1 Mtoe (million tonnes of oil equivalent) $\approx 4 \times 10^{7}$ GJ.................... 1

Figure 1.2 : Total global power capacity of solar PV from 2005 to 2015 [10]. ...... 4

Figure 2.1 : Cross-section of a typical solar cell [68] ..................................... 13

Figure 2.2 : Solar cell classification based on efficiency and cost [69].............. 14

Figure 2.3 : Development of best research-cell efficiencies [73] ...................... 15

Figure 2.4 : Solar cell equivalent circuit with parasitic series and shunt resistance [68] 17

Figure 2.5 : Solar cell $I-V$ and $P-V$ characteristic curves [68]. 18

Figure 2.6 : Schematic of standard solar cell performance measurement setup with standard illumination and temperature [68]. 20

Figure 2.7 : Crystalline silicon solar cells with (a) high 25\% efficiency PERL design [79] and (b) screen-printable design for commercial fabrication [80] ...... 21 Figure 2.8 : Amorphous silicon with a p-i-n structure [80]. 23

Figure 2.9 : Organic solar cells with (a) bilayer heterojunction structure and (b)

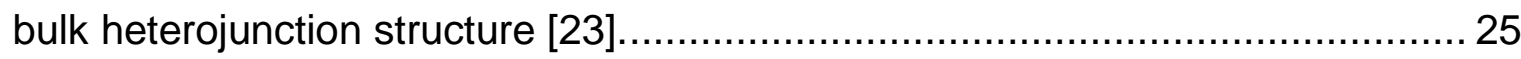

Figure 2.10 : All metal oxide based solar cell with (a) bilayer structure and

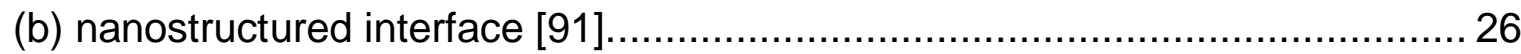

Figure 2.11: Crystalline silicon heterojunction solar cell [100]........................ 29

Figure 2.12 : Planar silicon / organic hybrid heterojunction solar cell [103] ........ 30 Figure 2.13 : Nanostructure interface silicon / organic hybrid solar cell [34]. The scale bar is $100 \mathrm{~nm}$.

Figure 2.14 : Silicon / organic hybrid solar cell with silver nanowire meshes top electrode [109]. 33

Figure 2.15 : Flexible ultra-thin $\mathrm{SOH}$ solar cell with front surface nanowire and back surface plasmonic Ag nanoparticles embedded in aluminum oxide [111]... 33 Figure 2.16 : Silicon / PEDOT:PSS hybrid solar cell with graphene quantum dots (GQDs) as photon down-converter [112]. 35

Figure 2.17 : Silicon / PEDOT:PSS hybrid solar cell with back junction structure [27]. 37 
Figure 2.18 : Silicon / transition metal oxide heterojunction solar cell with planar heterojunction interface [54].

Figure 2.19 : Silicon / transition metal oxide heterojunction solar cell with textured heterojunction interface [97]. 39

Figure $2.20: \mathrm{TiO}_{2}$ as hole blocking TMO layer for n-type Si [95] (a) band structure, (b) device cross section and (c) operational principle. 40

Figure 2.21 : (a) Cross section of Sliver ${ }^{\circledR}$ solar cell structure in which Lambertian reflector is used to increase the active surface area and (b) optical image of Sliver ${ }^{\circledR}$ solar cell [123].

Figure 2.22 : SEM image of 3D solar cell structure [124, 125]. The individual 3D solar cell structure has $40 \mu \mathrm{m} \times 40 \mu \mathrm{m}$ base and $100 \mu \mathrm{m}$ height. 43

Figure 2.23 : Three different stacks of multiple large area conventional PV modules in vertical structure [128]. 45

Figure 3.1: Chemical structure of PEDOT:PSS [135]. Symbol "•" and "+" represent unpaired electron and positive charge.

Figure 3.2 : (a) Scheme of n-Si/PEDOT:PSS hybrid junction structure and (b) optical image of n-Si/PEDOT:PSS hybrid solar cell.

Figure $3.3: \mathrm{n}-\mathrm{Si} / \mathrm{PEDOT}:$ PSS structure based on (a) p-n and

(b) Schottky junction model. 49

Figure 3.4 : Fabrication process of planar n-Si/PEDOT:PSS hybrid junction. ..... 51 Figure 3.5 : Measurement setup for reverse recovery transient characterization of n-Si/PEDOT:PSS hybrid junction. 53

Figure 3.6 : (a) Theoretical minority carrier distribution profiles of p-n junction under forward and reverse bias and (b) time-evolution of minority carrier distribution at $\mathrm{n}$-side after the reverse bias switching [78].

Figure 3.7 : Theoretical response of reverse recovery transient measurement for Schottky and p-n junctions. 55

Figure 3.8 : Determination of settling time $\left(\tau_{s}\right)$ duration. 56

Figure 3.9 : RRT curves for Si/PEDOT:PSS hybrid junctions with different $N_{D}$. The current for each sample is normalized to $I_{F} \approx 18.1-19.0 \mathrm{~mA} / \mathrm{cm}^{2}$ and $I_{R O} / I_{F} \approx 1.1$ 
Figure 3.10: RRT curves for the hybrid junction with $N_{D}=10^{16} / \mathrm{cm}^{3}$ at different forward bias injection levels $\left(I_{F}\right)$. The diode current is normalized to $I_{F}$. The settling times deduced are indicated by the diamond $(\diamond)$ marks.

Figure 3.11 : Lifetime $\tau_{p}$ dependence on injection level $I_{F}$, deduced for the hybrid junction with $N_{D}$ of $10^{16} / \mathrm{cm}^{3}$.

Figure 3.12 : Dark $J-V$ response of planar n-Si/PEDOT:PSS junction at different Si substrate doping concentrations. 63

Figure 3.13 : Equivalent circuit of a two-diode model of a p-n junction [68] 64

Figure 3.14 : Illuminated J-V response of planar n-Si/PEDOT:PSS junction at different Si substrate doping concentration. 68

Figure 4.1 : Band diagram of n-type Si/PEDOT:PSS heterojunction, for Si doping concentration $N_{D}=1 \times 10^{17} / \mathrm{cm}^{3}$. The electron affinity (EA), work function (WF) and ionization energy (IE) of the two materials are indicated. 77

Figure 4.2 : Cross-section of n-Si/PEDOT:PSS hybrid solar cell with conventional front junction (FJ) structure.

Figure 4.3 : Cross-section of n-Si/PEDOT:PSS hybrid solar cell with front junction and ARC compensated layer (FJ-ARC). 82

Figure 4.4 : Cross-section of n-Si/PEDOT:PSS hybrid solar cell with back junction (BJ) structure. 84

Figure 4.5 : Cross-section of n-Si/PEDOT:PSS hybrid solar cell with interdigitated back contact (IBC) structure. 85

Figure 4.6 : Influence of PEDOT:PSS thickness $t_{P}$ on (a) $J_{s c}$ and $V_{o c}$ and (b) FF and PCE of FJ solar cell with $N_{D}=10^{17} \mathrm{~cm}^{-3}$. 86

Figure 4.7 : Influence of $\mathrm{Si}$ doping concentration $\left(N_{D}\right)$ on FJ solar cell performance. 88

Figure 4.8 : Comparison of photovoltaic performance between simulation and experimental device as a function of Si doping concentration $\left(N_{D}\right)$. 89 Figure 4.9 : Influence of $t_{P}$ and $t_{A R C}$ on the (a) short circuit current density $\left(J_{s c}\right)$ and (b) open circuit voltage ( $\left.V_{o c}\right)$ of FJ-ARC hybrid cell with $N_{D}=10^{17} \mathrm{~cm}^{-3}$. 91

Figure 4.10 : Influence of $t_{P}$ and $t_{A R C}$ on the (a) fill factor (FF) and (b) power conversion efficiency (PCE) of FJ-ARC hybrid cell with $N_{D}=10^{17} \mathrm{~cm}^{-3}$. 92 
Figure 4.11 : Influence of $t_{s i}$ and $N_{D}$ on the (a) short circuit current density $\left(J_{s c}\right)$ and $(b)$ open circuit voltage $\left(V_{o c}\right)$ of BJ solar cell.

Figure 4.12 : Influence of $t_{S i}$ and $N_{D}$ on the (a) fill factor (FF) and (b) power conversion efficiency (PCE) of the BJ cell. 95

Figure 4.13 : Influence of PEDOT:PSS thickness $\left(t_{P}\right)$ on the BJ cell performance.

Figure 4.14 : The IBC hybrid solar cell performance (a) as a function of $W_{\text {cathode }}$ for a given $W_{\text {anode }}=5000 \mu \mathrm{m}$ and $W_{\text {gap }}=60 \mu \mathrm{m}$ and (b) as a function of $W_{\text {gap }}$ for a given $W_{\text {anode }}=5000 \mu \mathrm{m}$ and $W_{\text {cathode }}=60 \mu \mathrm{m}$. 98

Figure 4.15 : Influence of $W_{\text {anode }}$ on IBC solar cell performance with

$W_{\text {cathode }}=W_{\text {gap }}=60 \mu \mathrm{m}$

Figure 4.16 : I-V curves of n-Si/PEDOT:PSS hybrid solar cell with front junction (FJ), front junction with ARC compensated layer (FJ-ARC), back junction (BJ) and interdigitated back contact (IBC) structure. 101

Figure 5.1 : Schematics of (a) n-type Si/organic heterojunction solar cell and (b) n-type Si/metal oxide heterojunction solar cell. 106

Figure 5.2 : Energy level alignment of $\mathrm{MoO}_{\rtimes} / \mathrm{CBP}$ interface (left) and the model for hole injection and the transfer mechanism (right) [174]. 111

Figure 5.3 : A model of Si/TMO heterojunction solar cell based on n-type TMO with an electron blocking property [99]. 112

Figure 5.4 : (a) Fabrication process of baseline $\mathrm{n}-\mathrm{Si} / \mathrm{MoO}_{x}$ heterojunction solar cell and (b) optical image of $n-\mathrm{Si} / \mathrm{MoO}_{x}$ solar cell. 114

Figure 5.5 : Illuminated $\mathrm{J}-\mathrm{V}$ curve of $\mathrm{n}-\mathrm{Si} / \mathrm{MoO}_{x} / \mathrm{ITO}$ solar cell with various $\mathrm{n}-\mathrm{Si}$ substrate doping concentrations. 115

Figure 5.6 : Effect of $N_{D}$ on the dark $J-V$ curve of $n-S i / M_{0} O_{x}$ based solar cell... 118 Figure 5.7 : Fabrication process of $\mathrm{Si} / \mathrm{MoO}_{x}$ heterojunction solar cell with PEDOT:PSS as an electrode. (a) Direct contact between $\mathrm{MoO}_{x}$ and PEDOT:PSS and (b) thin evaporated ITO layer incorporated between $\mathrm{MoO}_{x}$ and PEDOT:PSS.

Figure 5.8 : Illuminated $J-V$ curves of $n-S i / M_{2} /$ PEDOT:PSS solar cell and $n$ $\mathrm{Si} / \mathrm{MoO}_{\times} / \mathrm{ITO} / \mathrm{PEDOT}: \mathrm{PSS}$ solar cells with different treatment conditions. 120 
Figure 5.9 : Dark J-V curves of $n-S i / M_{x}{ }_{x} /$ PEDOT:PSS solar cell and n$\mathrm{Si} / \mathrm{MoO}_{x} / \mathrm{ITO} / \mathrm{PEDOT}: \mathrm{PSS}$ solar cells with different treatment conditions. 123

Figure : $5.10 \mathrm{n}-\mathrm{Si} / \mathrm{MoO}_{x} / \mathrm{ITO}$ solar cell structures (a) without barrier layer and (b) with evaporated thin ITO $(5 \mathrm{~nm})$ as a barrier layer. The structures shown are not to scale, and the $\mathrm{MoO}_{x}$ and ITO layer thicknesses have been exaggerated. .... 125 Figure : 5.11 (a) Illuminated J-V curves and (b) dark J-V curves of $n-S i / M_{0} \times / I T O$ solar cells fabricated with or without thin ITO barrier layer 127

Figure 5.12 : n-Si/MoO ${ }_{x} / \mathrm{ITO}$ solar cell (a) without a native oxide and (b) with a thin layer of Si native oxide.

Figure 5.13 : (a) Illuminated and (b) dark $J-V$ curves of $n-S i / M O_{x} / I T O$ solar cell with and without native oxide at the $\mathrm{n}-\mathrm{Si} / \mathrm{MoO}_{\mathrm{x}}$ interface. 131

Figure 6.1 : Schematic of (a) conventional planar solar cell and (b) PV-Tower cell.

Figure 6.2 : Distinction between (a) light trapping concept based on arrays structure and (b) direct increase of active surface area by PV-Tower. 142 Figure 6.3 : (a) conventional Si wafer solar cell with patterned electrodes on both surfaces and cut along the blue dotted lines to form PV-Towers, (b) Individual vertically aligned PV-Tower solar cell and (c) Multiple PV-Tower solar cells connected in series on a rigid insulating substrate (not to scale). 143

Figure 6.4 : Area improvement factor (IF area) for PV-Tower solar cells with a square cross-section and with various tower heights. 146

Figure 6.5 : Study of PV-Tower cell response to light with different incident directions.

Figure 6.6 : Top view of PV-Tower with $200 \mu \mathrm{m} \times 200 \mu \mathrm{m}$ cross section and definition of the angular direction.

Figure 6.7 : Photogeneration profiles for the light with different incident angles. 151 Figure 6.8 : Power output of a PV-Tower with $2.5 \mathrm{~cm}$ height. 152 Figure 6.9 : Current flow through the metal grids on the side walls of PV-Tower.

Figure 6.10 : Influence of PV-Tower height on its power output, with a tower cross section of $200 \mu \mathrm{m} \times 200 \mu \mathrm{m}$. 157 
Figure 6.11: The area and power improvement factors as a function of tower height for a PV-Tower with $200 \mu \mathrm{m} \times 200 \mu \mathrm{m}$ square cross section. ................ 159 Figure 6.12 : PV-Tower with n-Si/PEDOT:PSS heterojunction solar cell. ......... 160 Figure 6.13 : Power output of a PV-Tower with $2.5 \mathrm{~cm}$ height that adopts $\mathrm{Si}$

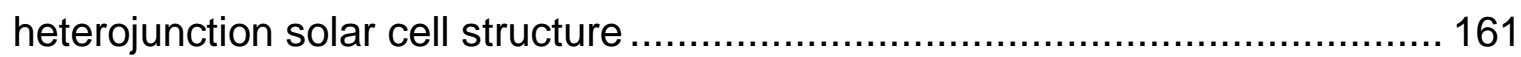




\section{List of Tables}

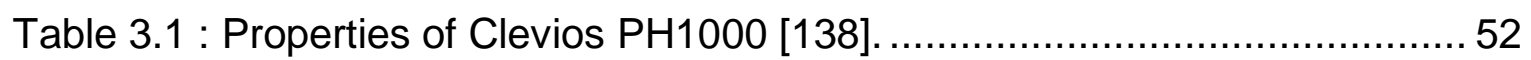

Table 3.2 : Settling time $\tau_{s}$ and extracted carrier lifetimes $\tau_{p}$ for the hybrid

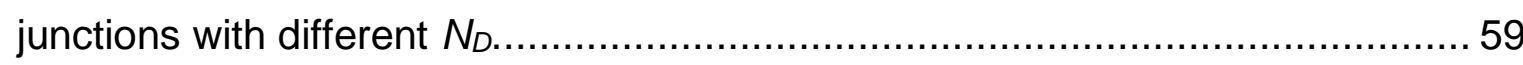

Table 3.3 : Two-diode model parameters used to fit dark $J$ - $V$ characteristic for the

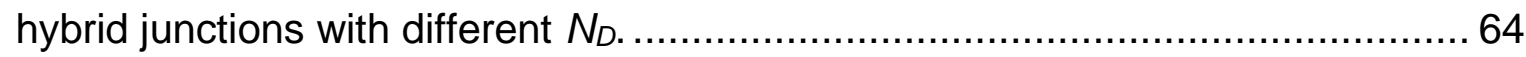

Table 3.4 : PV Parameters of n-Si/PEDOT:PSS hybrid solar cell at different Si substrate doping concentrations. "Best" sample refer to the sample with the highest PCE for a particular $N_{D}$. The average value is calculated from four

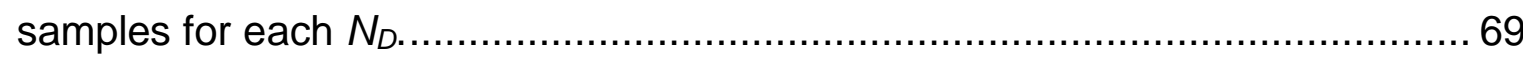

Table 4.1 : The electronic properties of PEDOT:PSS ................................. 78

Table 4.2 : Comparison of hybrid Si/PEDOT:PSS solar cell PV parameters for planar FJ cell, FJ-ARC cell, BJ cell and IBC cell. All cells are based on a planar structure with $\mathrm{ND}_{\mathrm{D}}=10^{17} \mathrm{~cm}^{-3}$. 102

Table 5.1 : n-Si/MoOx/ITO solar cell device as a function of n-Si donor concentration.

Table 5.2 : Photovoltaic parameters of $n-S i / M_{\star} /$ PEDOT:PSS solar cell and $n$ $\mathrm{Si} / \mathrm{MoO}_{x} / \mathrm{ITO} / \mathrm{PEDOT}: \mathrm{PSS}$ solar cells with different treatment conditions.

Table 5.3 : Photovoltaic parameters for $n-S i / M O_{x} / I T O$ solar cell with or without thin ITO barrier layer. 128

Table 5.4 : Photovoltaic parameters of $n-S i / M_{0 O} \times / T O$ solar cell with and without native oxide at the $\mathrm{n}-\mathrm{Si} / \mathrm{MoO}$ interface. 


\section{Chapter 1. Introduction}

\subsection{Background and motivation}

World 1 total primary energy supply (TPES) from 1971 to 2014 by fuel (Mtoe)

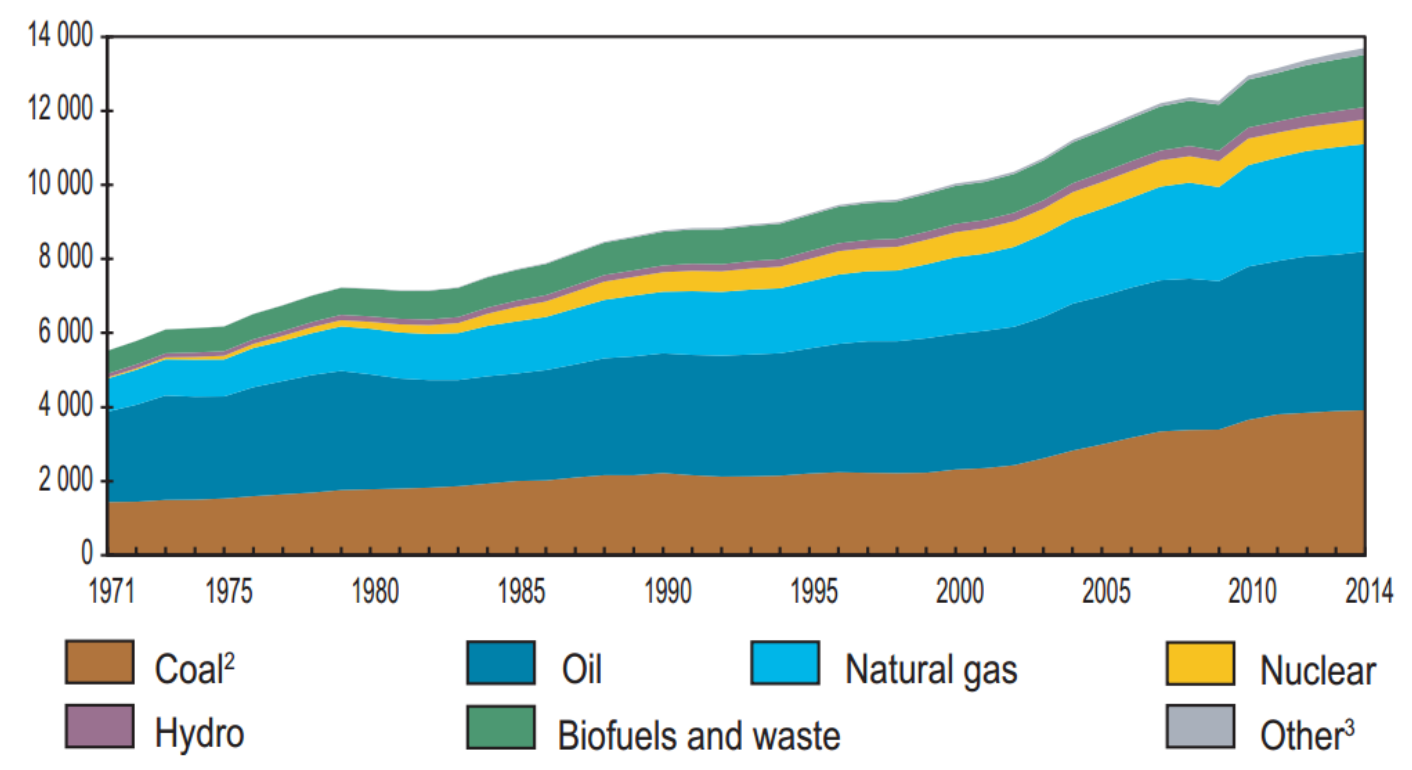

Figure 1.1 : World total primary energy supply from 1971 to 2014 in a unit of Mtoe/year [1]. 1 Mtoe (million tonnes of oil equivalent) $\approx 4 \times 10^{7} \mathrm{GJ}$.

The global energy demand increases as the world population grows, and communities' standard of living develops. Figure 1.1 shows the total primary energy supply, where it can be seen that the annual energy consumption has increased from $6 \times 10^{3}$ to $13.7 \times 10^{3}$ Mtoe/year from 1971 to 2014 [1]. Most of the current world energy consumption is fulfilled by fossil fuel based energy sources such as coal, oil and natural gas, which are non-renewable. These fossil fuels energy sources are going to be scarce or even depleted within the next 30-100 years [2], resulting in a severe energy crisis. In the long term, fossil fuels scarcity will also make fossil fuel 
energy more expensive due to high energy demand and low energy supply, subsequently, impede economic and social development [3]. Therefore, development of renewable energy harvesting technologies as a replacement for fossil fuels combustion will be essential to sustain continuous economic and social growth. In addition, fossil fuels combustion also releases a large amount of greenhouse gases (GHGs) such as carbon dioxide $\left(\mathrm{CO}_{2}\right)$ to the earth atmosphere, that will result in climate change [4]. It is estimated that $70 \%$ of human-caused GHGs are emitted by the energy sector, primarily $\mathrm{CO}_{2}$ from fossil fuels combustion [5]. The climate change will cause detrimental effects such as global average temperature increases, increased frequency of extreme weather events, ocean acidification, polar ice melting and sea level rises, which will be detrimental to human and the environment $[3,6]$. In order to address the energy crisis and climate change issues, it is critical to develop alternative energy sources that are both renewable, cost effective and safe for the environment.

Among renewable energy sources, solar energy is attractive since it is clean, environmentally safe, abundant, free and widely available. While it currently contributes only a small amount to the total energy supply [1], solar energy has good potential to meet future energy demand due to the high amount of solar energy radiation available in comparison to the expected future total energy consumption [7]. A realistic and practical amount of land is available to fulfill total energy demand by utilizing solar energy with currently available technology [8, 9]. 120,000 TW of solar power is available on the Earth from the sun [9]. Based on 
$10 \%$ efficiency solar module, solar PV installation with an area of 1 million $\mathrm{km}^{2}$ is required to supply electricity to the whole world [9], which is equivalent to about $0.7 \%$ of Earth land surface area. On the other hand, other renewable energy sources such as geothermal, hydroelectric, wind, and biomass have small utilizable energy potential, which is estimated to be much less than expected future energy consumption [9]. Therefore, developing solar energy is an effective way to provide alternative renewable energy supply and mitigate climate change.

Active solar energy technologies consist of mainly solar thermal [8] and solar photovoltaic (PV) [8]. Among them, solar PV is attractive since it can directly convert solar energy into electricity. Figure 1.2 shows the total global power capacity of solar PV from 2005 to 2015 [10]. The global solar PV capacity grew at a significant rate from $5.1 \mathrm{GW}$ in 2005 to $227 \mathrm{GW}$ in 2015 . In comparison to other renewable energy sources, solar PV also exhibits the highest annual growth rate of $28 \%$ in power capacity, higher than wind energy of $17 \%$ and solar thermal of 9.7\% [10]. International Energy Agency (IEA) predicts that solar PV will account for $11 \%$ of total global electrical energy supply in 2050 [7]. In summary, solar energy is expected to be a very important and promising renewable energy source in the future. 


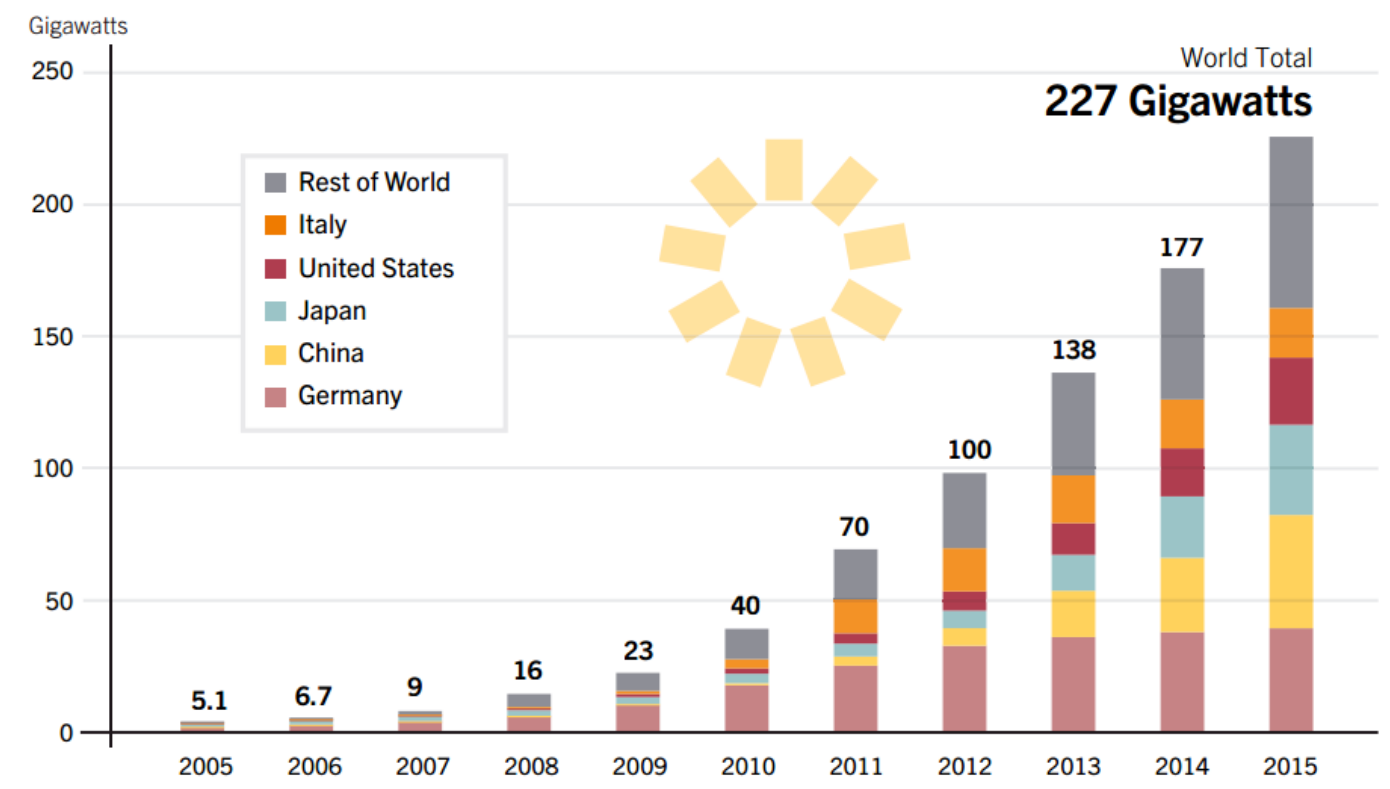

Figure 1.2 : Total global power capacity of solar PV from 2005 to 2015 [10].

However, despite its advantages, solar generated electricity is significantly more expensive compared to other energy sources, which limits its contribution toward world energy share at the moment. While the solar energy itself is free, the costs required to fabricate solar PV cell is quite high due to expensive materials and processes required. Multijunction solar cell based on GaAs and InP materials can convert $38.8 \%$ of light energy into electricity over a small area [11] but requires very expensive material and process. Physical availability of In based material also prevents its widespread use. Currently, the commercial PV market is based on the widely available material $\mathrm{Si}$, which is processed with conventional diffused single p-n homojunction technology. Such single junction Si based solar cell can reach high power conversion efficiency (PCE) of $25 \%[12,13]$ which is already close to the theoretical limit of 30\% [14] for single junction Si cell. The Si solar cell has high efficiency due to high quality monocrystalline Si material used, which has high 
carrier mobility and long minority carrier lifetime. In addition, the fabrication of conventional $\mathrm{Si}$ solar cell leverages on the mature technologies that have been developed and refined for the Si complementary metal-oxide-semiconductor (CMOS) technology. However, high temperature and high vacuum processes conditions are required in the fabrication of the high efficiency Si solar cell [15]. For that reason, intensive research is needed to render solar PV more cost effective to serve as a practical renewable energy source.

As an alternative to inorganic Si solar cell, the organic solar cell has been actively developed through comprehensive researches in the past decades, due to their low material cost, flexibility and solution based processing [16-23]. The latter characteristic allows low temperature processing at high throughput and over a large area. However, the performance of organic solar cell so far is limited by its poor electronic properties such as low mobility and low exciton diffusion length [24, 25]. This prompted the development of the concept of hybrid Si/organic solar cell that combines the advantages of inorganic and organic materials to produce a high performance solar cell with a simple solution based processing [26-33]. The organic materials are used as selective carrier contact (SCC) to form rectifying junction with Si material that acts as the light absorber. While various organic materials have been used to form hybrid Si/organic solar cells, a conjugated polymer poly(3,4ethylenedioxythiophene) : polystyrenesulphonate (PEDOT:PSS) emerges as most successful SCC for $n$-Si based solar cells that can yield high efficiency of $14-17 \%$ $[27,28,34]$. Despite its success, the nature of rectifying junction between $\mathrm{n}-\mathrm{Si}$ and 
PEDOT:PSS is not well understood [35-38]. As a conjugated polymer PEDOT:PSS exhibits both semiconductor and metallic characteristics which result in a contradictory interpretation of n-Si/PEDOT:PSS junction as both $p-n$ and Schottky junctions $[28-30,39,40]$. Ascertaining the nature of n-Si/PEDOT:PSS junction is necessary to accurately model and optimizes hybrid Si/PEDOT:PSS solar cell. Previously, the PCE of conventional front hybrid Si/PEDOT:PSS solar cell has been limited by parasitic absorption in PEDOT:PSS. This has been addressed by employing back junction structure in which PEDOT:PSS is located at the back of the solar cell, away from the incident light, resulting in high PCE of $16-17 \%[27,41]$. Apart from organic materials based SCCs, SCCs based on transition metal oxides (TMO) have recently attracted an interest since they offer higher transparency as compared to organic materials, and wider choices for material deposition, including evaporation [42, 43], atomic layer deposition [44] as well as solution based process [45-51]. Si/TMO solar cell with PCE of $15-18.8 \%$ [52-54] has recently been reported. Overall, Si heterojunction solar cells with organic and transition metal oxide based selective carrier contact layer are very promising and potentially suitable for practical application.

Apart from cost-effectiveness issue, the growing need to provide energy harvesting capability for Internet-of-Things sensor node (IOT-SN) application [55-58] drive the need to develop solar cell concept that can generate high power over small footprint area [55, 59-62]. Current approaches for compact energy autonomous loT-SN rely on energy management [63-66], physical layout design [59] and using non- 
conventional solar cell $[58,60,67]$. Development of solar cell concept that can harvest light energy with a large active surface area over small footprint is very crucial for this emerging important solar application.

\subsection{Objectives}

In this project, Si heterojunction hybrid solar cells based on organic and transition metal oxide selective carrier contacts are studied. The scope of this research work includes the design, simulation, fabrication and characterization of Si/organic hybrid solar cell and Si/transition metal oxide heterojunction solar cell with various device architectures. The objectives of this study are summarized as follows:

(i) Fabricate and characterize planar Si/PEDOT:PSS hybrid junction to study the junction characteristic. Reverse recovery characteristics of hybrid junction are investigated at various $\mathrm{Si}$ substrate doping concentrations and different forward current injection levels. Dark I-V and photovoltaic characteristics are also studied to verify the junction characteristic.

(ii) Simulate Si/PEDOT:PSS hybrid solar cell with four different device architectures using numerical device simulation approach. The four device architectures are conventional front junction, back junction, interdigitated back contact, and front junction with anti-reflection-coating (ARC) compensated layer. We study the effects of $\mathrm{Si}$ substrate 
thickness, doping, ARC layer and contact design for these various cell architectures to optimize their performance.

(iii) Fabricate $\mathrm{Si} /$ molybdenum oxide heterojunction solar cell with various $\mathrm{Si}$ substrate doping concentrations, the use of PEDOT:PSS and ITO electrodes, the presence of water barrier layer, and native oxide passivation condition.

(iv) Simulate PV-Tower cell based on Si solar cell with the high active surface area over a given footprint. The effects of Si substrate cross section and doping concentration on the performance of the PV-Tower cell are investigated at various light incidence angles. The effect of height scaling to ohmic losses is also analyzed.

\subsection{Major contribution of the thesis}

(i) We characterized the junction behavior of $n$-Si/PEDOT:PSS hybrid solar cell fabricated with a simple spin coating process. Using reverse recovery transient (RRT) and DC-IV measurement we found that the hybrid solar cell does not act like a Schottky junction as it is commonly assumed. We found that n-Si/PEDOT:PSS junction behaviour is consistent with $n-p^{+}$junction model. 
(ii) Using $n-p^{+}$junction model, we simulated the performance of $n-S i / P E D O T: P S S$ hybrid solar cell with different device architectures. We studied four device architectures, including conventional front junction cell (FJ), back junction cell (BJ) cell, interdigitated back contact cell (IBC) and front junction cell with ARC compensated layer (FJ-ARC). To the best of our knowledge, there is no such comprehensive study and comparison of n-Si/PEDOT:PSS hybrid solar cells with the various cell architectures. In particular, our proposed FJ-ARC cell which can deliver similar performance as the BJ cell has not been reported and investigated before.

(iii) We also investigated $\mathrm{n}$-Si/transition metal oxide solar cell using molybdenum oxide $\left(\mathrm{MoO}_{\mathrm{x}}\right)$ as selective carrier contact layer at low substrate temperature. The effect of different electrodes (PEDOT:PSS and sputtered ITO), barrier layer, doping concentration and interfacial native oxide are investigated.

(iv) We proposed and developed PV-Tower cell concept which offers a huge active surface area, yet occupies only a small footprint. The height of PV-Tower cell can be substantially increased to enhance the output power while its multiple surface area allows efficient diffuse light collection. We simulated the performance of PVTower using device simulation software. Then we also investigate the effect of ohmic losses at PV-Tower electrodes, which is found to scale as a function of the cubic height of the tower. We also demonstrated that PV-Tower cell could adopt Si heterojunction solar cell structure. 


\subsection{Organization of the thesis}

This thesis consists of seven chapters organized as follows:

Chapter 1: Background, motivation, objectives and major contributions of the project are presented.

Chapter 2: The fundamental principles of solar cell operation are presented. A literature review of conventional Si based solar cells, organic solar cells, hybrid solar cells, Si/transition metal oxide heterojunction solar cells and PV-Tower concepts are presented.

Chapter 3: Planar hybrid solar cells based on $\mathrm{n}-\mathrm{Si}$ and PEDOT:PSS are fabricated and characterized to study the nature of the junction characteristic. Reverse recovery transient characteristics are investigated for the hybrid junction with different Si substrate doping concentrations and forward bias injection levels. The nature of the junction as deduced from the transient characterization is further validated by the study of dark and illuminated I-V characteristic.

Chapter 4: Simulation of the performance of hybrid solar cell based on n-Si and PEDOT:PSS is conducted with different device architectures. Four hybrid solar cell device architectures are studied and compared, which include the conventional front junction (FJ) cell, back junction (BJ) cell, interdigitated back contact (IBC) cell and front junction structure with ARC compensated layer (FJ-ARC) cell. 
Chapter 5: Si/transition metal oxide heterojunction solar cell based on $\mathrm{n}-\mathrm{Si}$ and molybdenum oxide is studied experimentally. The effects of Si substrate doping concentration, use of different electrode such as PEDOT:PSS or ITO, the presence of water barrier layer on top of $\mathrm{MoO}_{x}$ and the influence of native oxide at $n-\mathrm{Si} / \mathrm{MoO}_{x}$ interface is presented.

Chapter 6: The concept of PV-Tower cell and its advantage in terms of large power generated over a given footprint area is presented. Device simulation of PV-Tower cell is also carried out with conventional and $\mathrm{Si}$ heterojunction solar cell structure. Moreover, analysis of ohmic losses with height scaling is also discussed.

Chapter 7: A general summary of the work done and recommendation for future work are presented. 


\section{Chapter 2. Literature Review}

This chapter reviews the literature on solar cells fundamentals, silicon solar cells, organic solar cells, metal oxide solar cells and silicon heterojunction solar cells. The chapter starts by introducing solar cell fundamentals and a general summary of current materials and technologies developed. Subsequently, various categories of solar cells including silicon solar cells, organic solar cells, and metal oxide solar cells are presented in term of material properties and processing requirement. We will then present the ideas that drive the development of Si heterojunction solar cell that leverages on the good electrical properties of Si and the processing advantage of organic and metal oxide materials. Current development of Si heterojunction solar cell with organic and metal oxide based selective carrier contact layer will be presented. Moreover, we will also present the advance of solar cell concept for small footprint application.

\subsection{Solar cells fundamentals}

\subsubsection{Introduction of solar cell operation}

A solar cell or photovoltaic (PV) cell is a device that can convert solar energy directly into electrical energy. The solar cell consists of at least a light absorber, selective carrier contact (SCC) and electrodes that are connected to an external load. Figure 2.1 shows a typical cross section of a solar cell [68], with a p-type base as the light absorber and n-type emitter as the electron accepting SCC, forming a 
p-n junction. When photons with energy higher than the material bandgap are absorbed, electron - hole pairs are generated inside the light absorber. These electron - hole pairs will recombine within a time period of minority carrier lifetime unless they can be separated at the interface with a material that has asymmetrical electronic properties with respect to the light absorber. Such material is called a selective carrier contact (SCC) that allows one type of carrier to pass, while blocks the other carrier with opposite polarity. The n-type emitter, in this case, allows the electron to pass while blocks the hole, creating separated charge carriers which will become majority carriers in the respective layers. The carriers are then driven toward respective positive and negative contacts to flow toward external circuit as photocurrent. When the cell is set at open circuit, it establishes a photovoltage and will deliver power when connected to an external load.

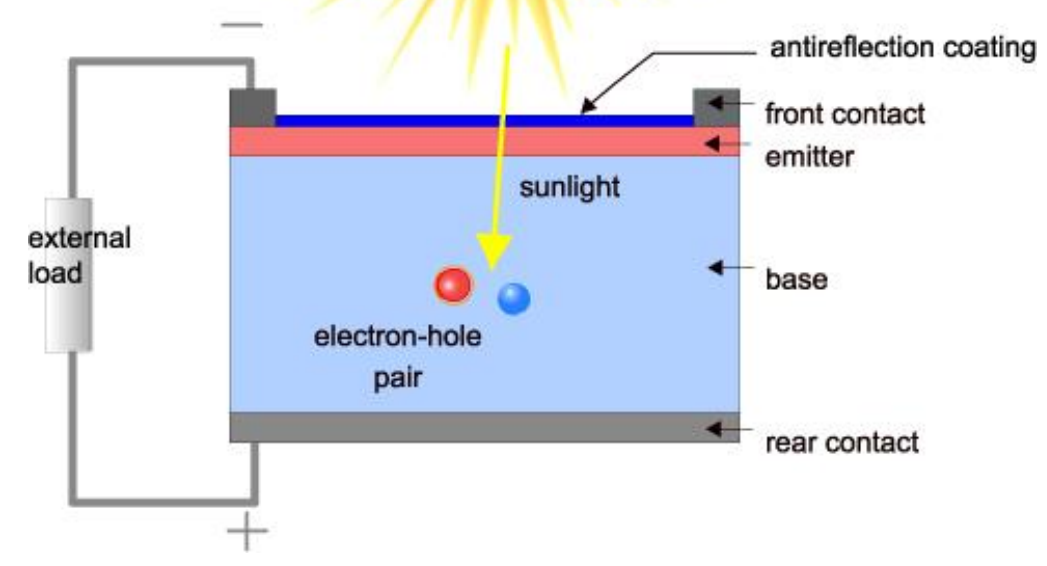

Figure 2.1 : Cross-section of a typical solar cell [68]. 


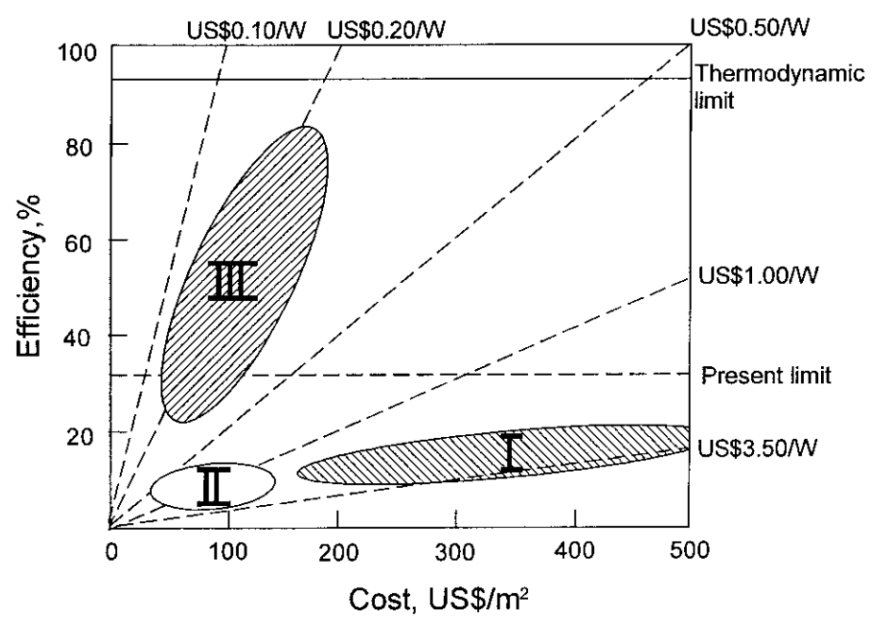

Figure 2.2 : Solar cell classification based on efficiency and cost [69].

Solar cells can be classified into three generations, as shown in in Fig. 2.2, based on its expected efficiency and cost [69]. The horizontal and vertical axis shows cost and efficiency, respectively. The dotted line shows combinations of cost and efficiency which yield constant cost / power output ratio. First generation solar cells are based on crystalline or multicrystalline bulk Si solar cells, which cover $>80 \%$ of commercial PV market at the moment [7]. First generation solar cells have high performance but limited by high material and processing cost. Since they are generally based on bulk $\mathrm{Si}$ and high temperature diffusion process, it is difficult for the cost to be lowered. Second generation solar cells are based on thin film solar cell technology using materials such as Cadmium-Telluride (CdTe), CopperIndium-Gallium-Diselenide (CIGS) or amorphous Si [70]. Second generation solar cells are of lower cost but also suffer from lower efficiency, and account for 10-15\% of current commercial PV market [70]. Third generation solar cells cover various emerging solar cell technologies which may include multijunction, hot carriers, multiple carrier excitations, nanostructure or tandem solar cells that aim to achieve 
single junction efficiency limit but yet maintaining lower manufacturing cost [69-72].

Si heterojunction solar cells with selective carrier contact layer belong to third generation solar cells, in which high efficiency of conventional Si solar cell is expected yet with cost reduction from low temperature process.

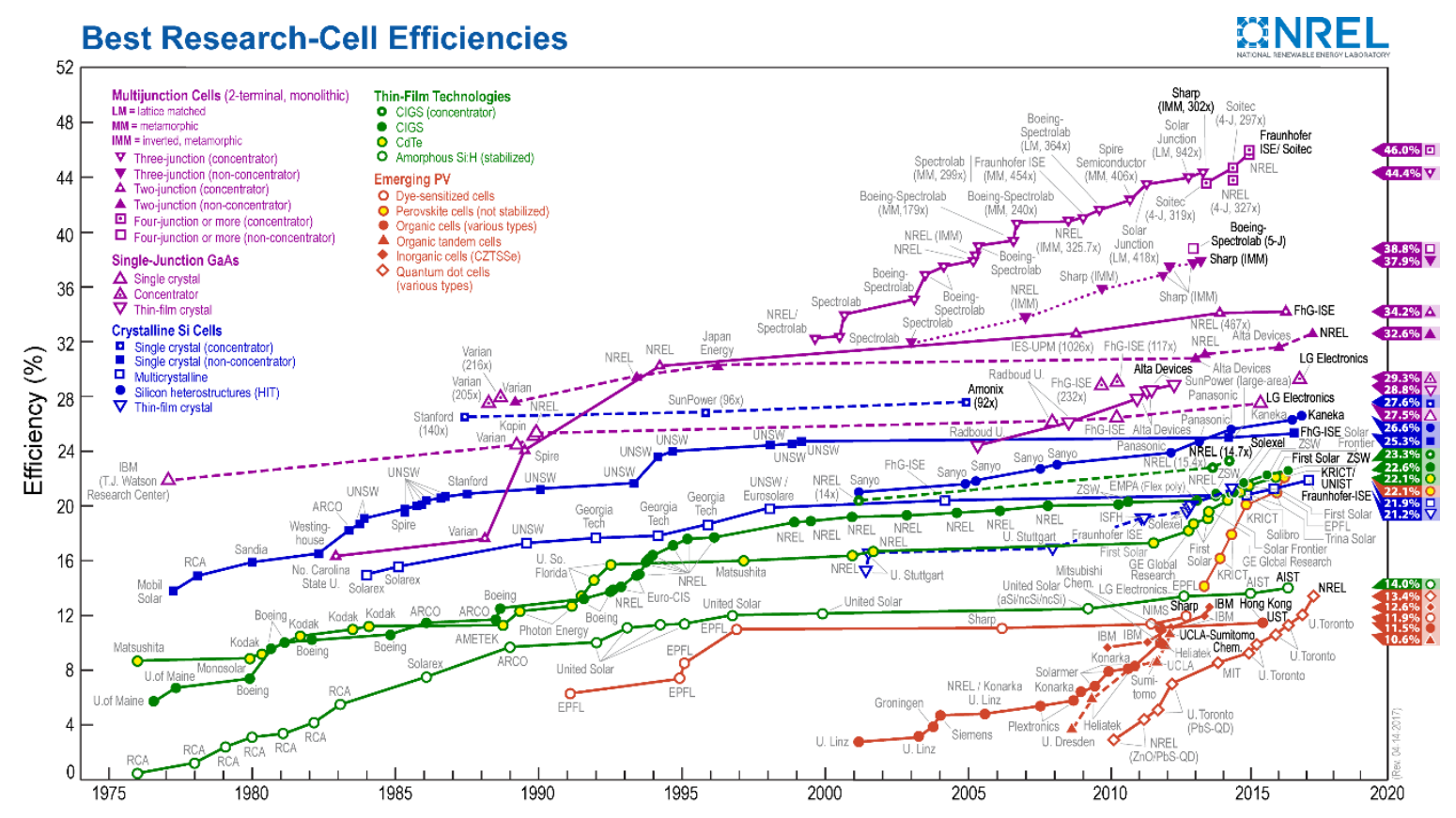

Figure 2.3 : Development of best research-cell efficiencies [73].

Figure 2.3 shows the record efficiencies reported of best research cells across the years with different solar cell materials and technologies. The highest performance is derived from multijunction cells based technology with power conversion efficiency (PCE) of 31.6 - 46.0\% [73]. In comparison, the single junction cells have much lower PCE, with GaAs exhibiting the highest single junction PCE at 29\% [11]. Multijunction and single junction GaAs cells are quite expensive in terms of material and technology, and hence have limited application in mass commercial solar cell 
market. Single crystal Si solar cell has an efficiency of $25.3 \%$ as reported by FhGISE [73], which does not improve much from the 25.0\% achieved since 1999 [13], due to relatively mature technology. The majority of commercial solar cells are based on single crystal Si but using much simpler process than the one adopted in the record efficiency cell. Crystalline Si solar cell with heterojunction intrinsic thin film (HIT) structure has been developed in the last decade and exhibits PCE $>26 \%$ for practical size device $[73,74]$. As for thin film based solar cells, the best efficiency is obtained for CIGS cell with a PCE $=22.6 \%$, which is close to that of CdTe cell of $22.1 \%$, and higher than the PCE of amorphous Si:H thin film solar cell of $14.0 \%$ [73]. Various emerging PV technologies such as dye-sensitized cells, organic cells, CZTSSe cells and perovskite cells have PCE > 10\% [73]. Amongst the emerging PV technologies, perovskite cells are interesting since it has a remarkable PCE $=$ $22.1 \%[73,75]$, although at present it still suffers from performance stability issue $[76,77]$. In summary, crystalline Si based research-cells still exhibit higher efficiency as compared to other cells technology, except for GaAs and multijunction cells. 


\subsubsection{Solar cell model}

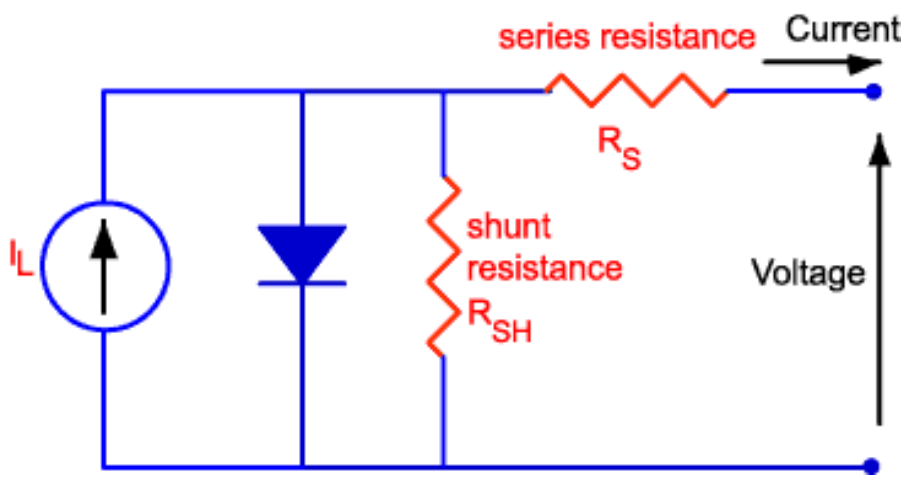

Figure 2.4 : Solar cell equivalent circuit with parasitic series and shunt resistance [68].

Figure 2.4 shows a solar cell equivalent circuit with parasitic series and shunt resistances included [68]. The photocurrent is represented by a constant current source component with value $I_{L}$ generated by light absorption and collected at the rectifying junction. To remove the influence of solar cell area $A$, typically the photocurrent is denoted by current density, $J_{L}=I_{L} / A$ for performance comparison. The dark current component of the solar cell is represented by a diode component that is parallel with the constant current source, with the positive terminal of the diode connected to the output terminal of the constant current source. The diode exhibits dark saturation current $J_{0}$ and an ideality factor $n$. The ideality factor depends on the recombination mechanisms taking place and measures the deviation of the diode behavior from the ideal diode characteristic [78]. A practical solar cell also exhibits series resistance $R_{\mathrm{s}}$ caused by solar cell materials resistivity as well as those of contacts, and shunt resistance $R_{\text {sh }}$ due to any current leakage. To achieve solar cell with high efficiency, it is important that $R_{\mathrm{s}}$ is as small as 
possible, while $R_{\text {sh }}$ is as large as possible. Based on the equivalent circuit, the current-voltage $J-V$ characteristic of a solar cell is similar to that of a diode with an ideality factor $n$, shifted with a photocurrent $J$, and with extra terms arising from shunt leakage and voltage modified due to the series resistance. It is given in Eq 2.1 where $q$ is the elementary charge, $T$ is the temperature in Kelvin and $k$ is the Boltzmann's constant.

$$
J=J_{L}-J_{0}\left(e^{\frac{q\left(V+J A R_{S}\right)}{n k T}}-1\right)-\frac{V+J A R_{S}}{R_{s h}}
$$

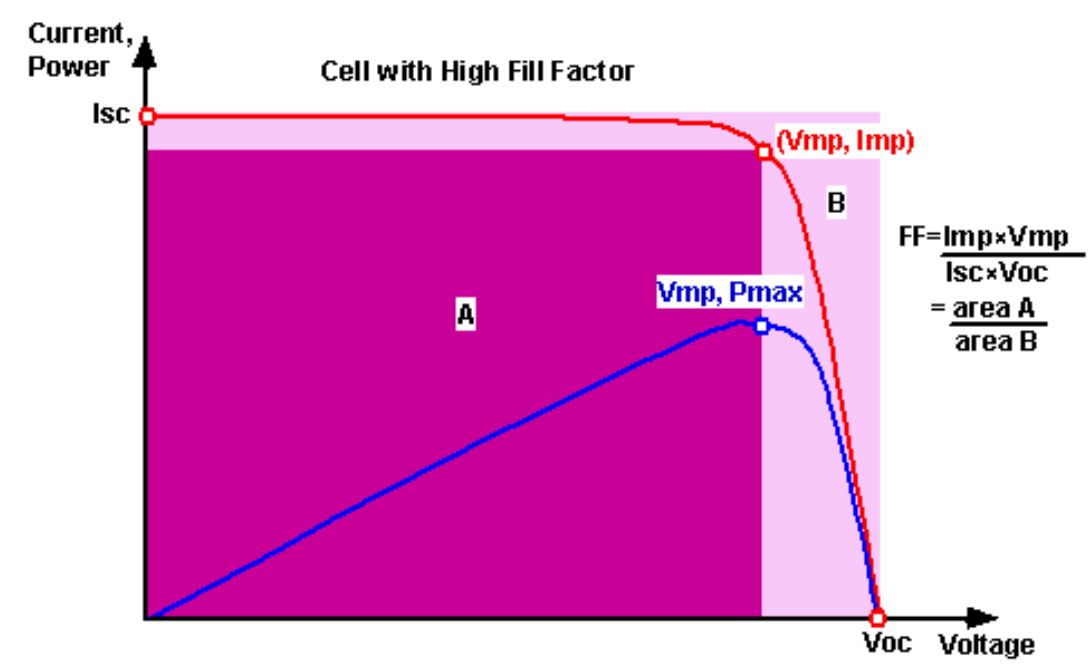

Figure 2.5 : Solar cell $I-V$ and $P-V$ characteristic curves [68].

Figure 2.5 shows typical current-voltage $(I-V)$ and power-voltage $(P-V)$ characteristic curves of a solar cell [68]. The solar cell performance is determined by four parameters, which are the short circuit current density $J_{s c}$, the open circuit voltage $V_{o c}$, fill factor FF and power conversion efficiency PCE. The $J_{s c}$ is the current density at $V=0$, and it represents the maximum photocurrent that can be collected 
from the solar cell under short circuit condition and is heavily dependent on the intensity of the incident illumination. The $J_{s c}$ is an integral of the product of external quantum efficiency $\eta(E)$ and the irradiance $I(E)$ of light with respect to the photon energy $E$ across the entire solar spectrum, as seen in Eq 2.2. The $\eta$ is the ratio of the number of carriers that the solar cell collects to the number of photons of a given energy incident on the solar cell from the outside, and it takes into account optical and electrical losses in the cell. The $V_{o c}$ is the terminal voltage of the solar cell at $l=0$, and it represents the maximum voltage at which the recombination current is equal to the photocurrent, resulting in a zero net current density. Solar cell only produces power when the voltage is somewhere between 0 and $V_{\text {oc. }}$. The $V_{\mathrm{oc}}$ is a function of $J_{\mathrm{sc}}$, dark saturation current $J_{0}$ and the diode ideality factor $n$ as shown in Eq 2.3. The maximum power point voltage $V_{\mathrm{mp}}$ represents the point in the $J-V$ characteristic where the output power is at its maximum $P_{\max }$, as can be seen from the $P-V$ curve. The maximum power point current $J_{m p}$ can be obtained from the $J-V$ curve with $V=V_{m p}$. The FF, defined as the ratio of the maximum power to the product of $V_{o c}$ and $J_{o c}$, and which measures the squareness of the $J$-V curve, is shown in Eq 2.4. Finally, the PCE which is defined as the ratio of the maximum output power $P_{\max }=V_{\mathrm{m}} J_{\mathrm{m}}$ to the input light power $P_{\text {in }}$ is given by Eq 2.5 .

$$
\begin{gathered}
J_{s c}=q \int(I(E) \eta(E)) d E \\
V_{o c}=\frac{n k T}{q} \ln \left(\frac{J_{s c}}{J_{0}}+1\right) \\
F F=\frac{V_{m} J_{m}}{V_{o c} J_{s c}}
\end{gathered}
$$




$$
P C E=\frac{V_{m} J_{m}}{P_{\text {in }}}=\frac{V_{o c} J_{s c} F F}{P_{\text {in }}}
$$

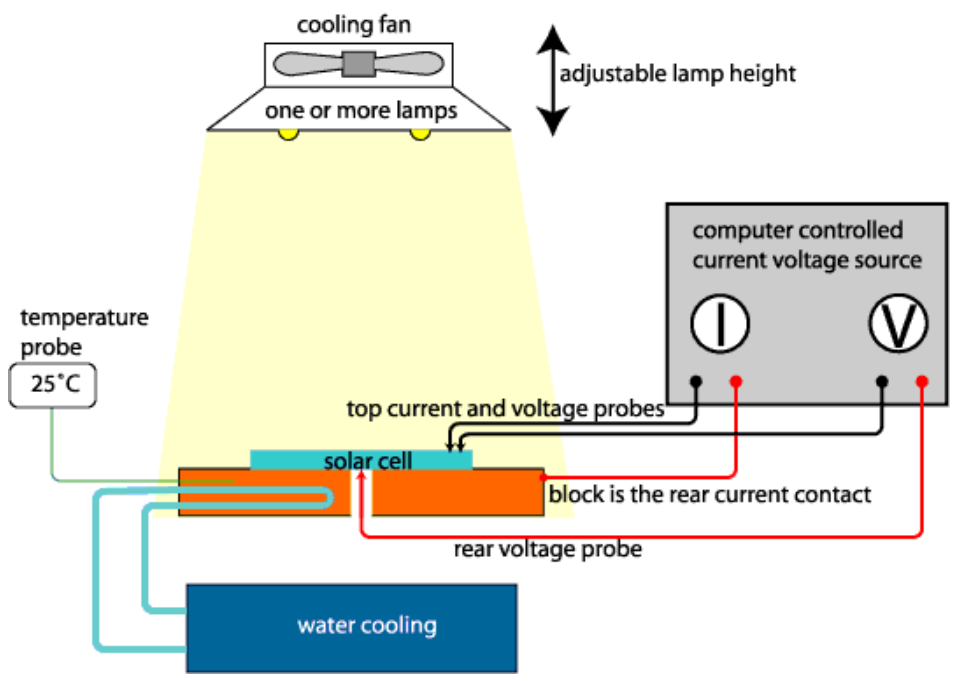

Figure 2.6 : Schematic of standard solar cell performance measurement setup with standard illumination and temperature [68].

Since the solar cell performance is dependent on the measurement conditions such as light intensity-spectrum $l(E)$ and temperature $T$, a standard measurement system is typically used for its characterization. Figure 2.6 shows the schematic of standard solar cell performance measurement [68] or a solar simulator. The input light power $P_{\text {in }}$ is provided by a lamp and optical filter system that delivers an air mass 1.5 (AM1.5) spectrum with a power intensity of $0.1 \mathrm{~W} / \mathrm{cm}^{2}$. The cell is placed on a temperature controlled substrate to maintain its temperature at around $25^{\circ} \mathrm{C}$. The J-V curves are measured by computer controlled I-V source with two or four probes connected to the solar cell sample. 


\subsection{Silicon, organic and metal oxide based solar cells}

\subsubsection{Silicon solar cells}

(a)

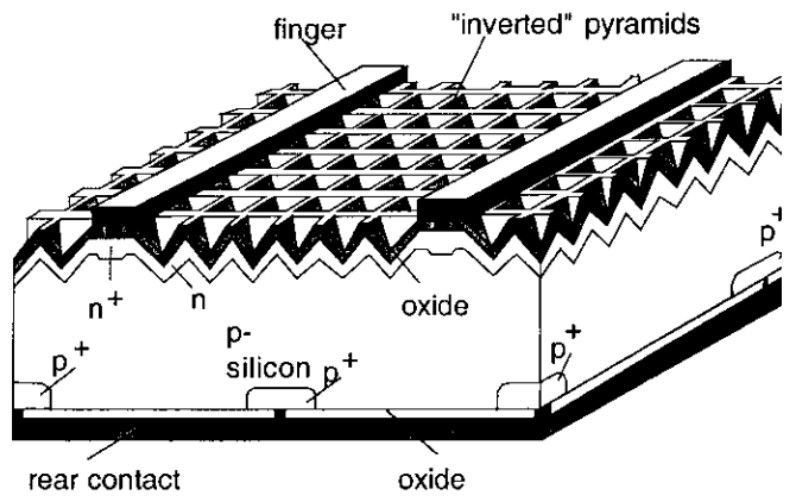

(b)

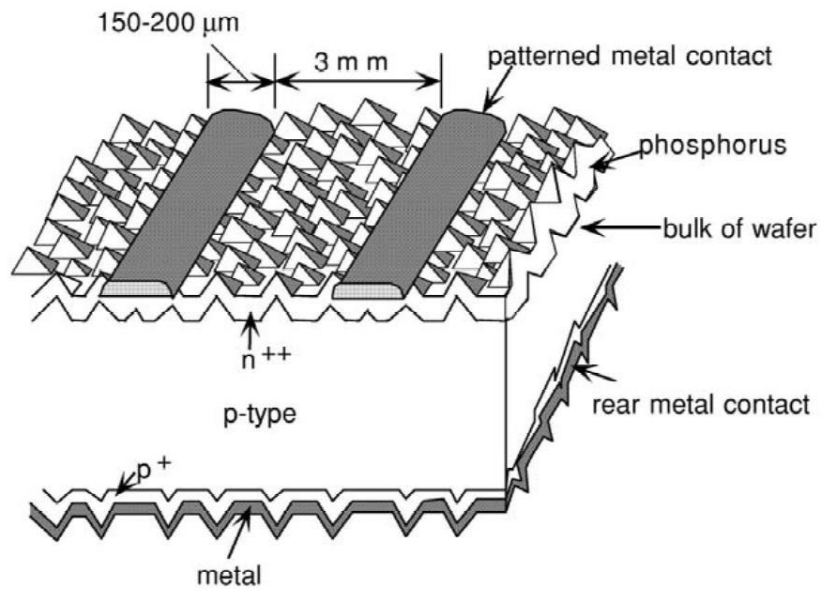

Figure 2.7 : Crystalline silicon solar cells with (a) high 25\% efficiency PERL design [79] and (b) screen-printable design for commercial fabrication [80].

The majority of commercial solar cells are based on crystalline Si since it has good optoelectronic properties, widely available source material, and mature technology. Fig. 2.7(a) shows crystalline silicon solar cell with $25 \%$ efficiency based on 
passivated emitter rear locally contacted (PERL) concept [79]. A base made of $p$ type $\mathrm{Si}$ is used as light absorber while an n-type emitter layer is used in the front to collect electrons. Locally diffused $\mathrm{n}^{+}$layers are formed below the top finger grid to form an ohmic contact between the n-layers and top grid. Most of the rear surface is passivated with a high quality oxide layer, and with a small opening formed for rear contact. Locally diffused $\mathrm{p}^{+}$layers act as a back surface field to collect holes as well as form ohmic contact. Inverted pyramid structure is used in the front to form antireflection structure. While high efficiency can be achieved with this structure, the processes used for making locally diffused and patterned contacts are not simple enough to be adapted to commercial process [79]. The majority of commercial crystalline Si based solar cells is based on screen-printable design [80] as shown in Fig. 2.7(b). An $\mathrm{n}^{++}$layer is diffused across the entire front surface as electron collecting layer, while a $\mathrm{p}^{+}$layer is formed on the entire back surface as a hole collecting layer by metal paste alloying. The formation of diffused $\mathrm{n}^{++}$layer on the front surface requires high temperature processing [15] that increases the cost required to form conventional crystalline Si based solar cell.

As an alternative to crystalline Si solar cell, amorphous silicon solar cell with a p-in structure as shown in Fig. 2.8 offers low temperature processing capability by using a plasma enhanced chemical vapor deposition process (PECVD) [81]. The ilayer of amorphous $\mathrm{Si}$ act as the main light absorber while the p-layer and n-layer of amorphous $\mathrm{Si}$ act as hole and electron collecting contacts, respectively. The $\mathrm{p}$ i-n structure creates a strong drift field at the intrinsic layer that assists charge 
separation. All the layers in the cell can be easily deposited by PECVD process. In terms of optical properties, amorphous $\mathrm{Si}$ is more advantageous compared to $\mathrm{Si}$ since it has a higher bandgap 1.7-1.9 eV that is more suitable for visible light absorption. As it is strongly absorbing, only a thin layer of amorphous $\mathrm{Si}$ is required to capture energy available in the sunlight effectively [82]. Due to its thin film structure, it can also be deposited on a low cost and flexible substrate, offering possibilities for various applications [83]. However, as amorphous Si has low carrier mobility and cannot absorb infrared light, its efficiency is limited. The best amorphous Si solar cell efficiency is around 14\% [73, 84], lower than most of commercial Si solar cell. While it is based on lower temperature deposition, the high quality PECVD process involved has relatively low throughput [85], and hence hinders its commercialization. Due to the above reasons, amorphous Si solar cell is currently not as cost effective as crystalline Si solar cell.

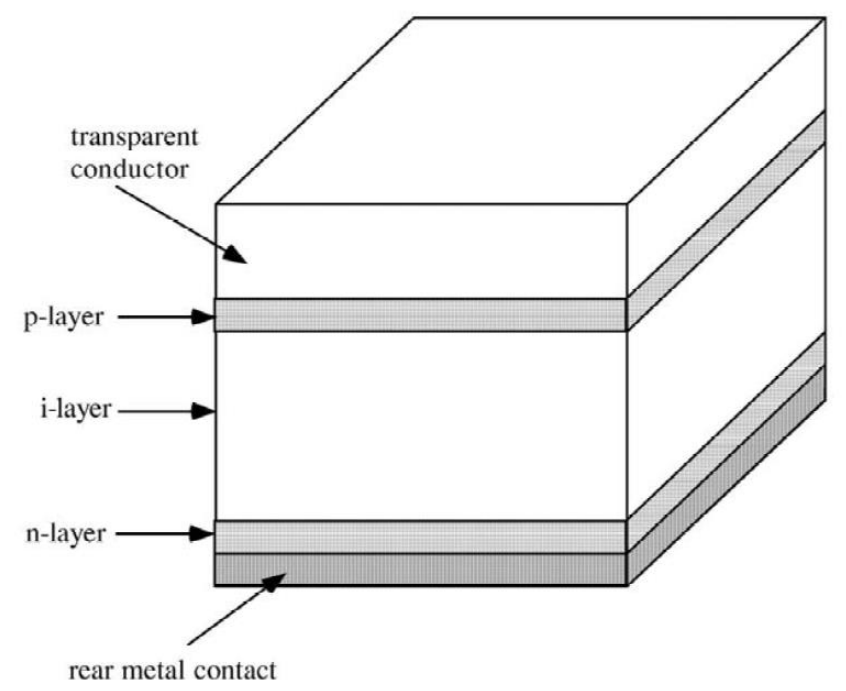

Figure 2.8 : Amorphous silicon with a p-i-n structure [80]. 


\subsubsection{Organic solar cells}

In contrast to crystalline and amorphous Si solar cells, organic solar cells as shown in Fig. 2.9 allow solution based processing that enables low temperature process, with high throughput and over a large area [23]. Since it is built using carbon based materials, it also offers a large selection of materials with a wide range of optical and electrical properties. A typical organic solar cell uses p-type conjugated organic polymer such as poly[2-methoxy-5-(3',7'-dimethyloctyloxy)-1,4-phenylenevinylene] (MDMO-PPV) [23] or poly(3-hexylthiophene-2,5-diyl) (P3HT) [86] as a hole collecting layer, and n-type organic molecule such as phenyl-C61-butyric acid methyl ester (PCBM) $[23,86]$ as an electron collecting layer. The simplest organic solar cell structure comprises a bilayer structure as shown in Fig. 2.9(a), where the p-type polymer layer forms a planar interface with n-type organic molecule layer [23]. Absorption of light in the p-type polymer generates electron-hole pairs, known as excitons, which are bound by strong Coulombic interaction. The excitons will diffuse into the bilayer junction to be separated as free holes in the p-type polymer and free electrons in the n-type organic layer. Due to the short exciton diffusion length $[87,88]$, most of the generated exciton cannot reach the heterointerface for collection and hence will recombine. In order to solve this problem, bulk heterojunction structure as shown in Fig. 2.9(b) [23] is used to shorten the distance required for carrier collection. However, due to the poor electronic transport properties, the best organic solar cell efficiency is still at $11.5 \%$ [89]. Another very important challenge is the performance stability, as most organic solar cells will significantly degrade under prolonged intense sunlight radiation or in the presence 
of ambient air [90]. Due to its low performance and instability, the organic solar cell at present has less commercialization success than Si solar cell.

(a)

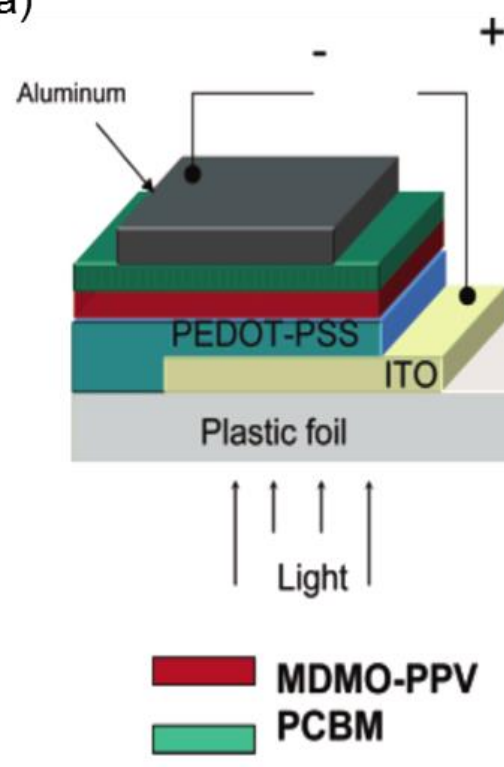

(b)

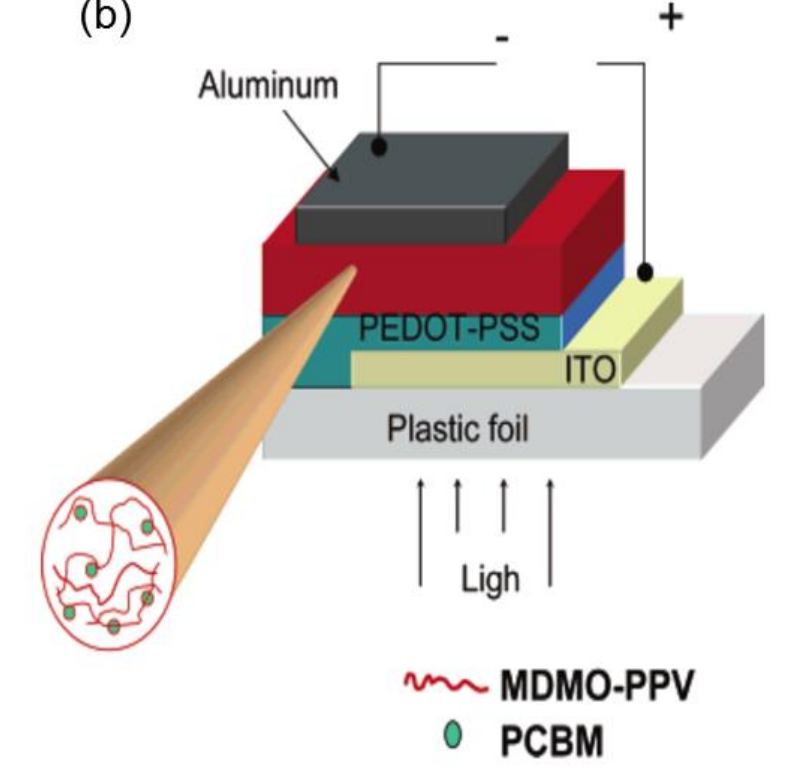

Figure 2.9 : Organic solar cells with (a) bilayer heterojunction structure and (b) bulk heterojunction structure [23].

\subsubsection{Metal oxide Solar Cells}

Recently, a class of solar cell based on all metal oxide materials emerges as an interesting alternative to organic solar cell [91, 92]. Similar to organic material, metal oxide also enables low temperature solution based processing [45, 93]. In fact, metal oxide has been used as selective carrier contact material for organic solar cell assisting in carrier collection [94]. In contrast to organic materials, many of the metal oxide materials are inherently stable in ambient air which is desirable for practical application [91]. Various metal oxides, such as $\mathrm{TiO}_{\times}$[95], $\mathrm{ZnO}_{\times}$[96], 
$\mathrm{MoO}_{\times}[43,52,53,97-99], \mathrm{V}_{2} \mathrm{O}_{\times}[43,99]$ or $\mathrm{WO}_{\times}[43,98,99]$ have large bandgaps $(>3 \mathrm{eV})$ that allow transparent front electron or hole collecting layer. Light absorbing metal oxides such as $\mathrm{Cu}_{2} \mathrm{O}$ and $\mathrm{SnO}$ in combination with transparent selective carrier contact metal oxide may form all metal oxide solar cell [91-93]. However, $\mathrm{Cu}_{2} \mathrm{O}$ also has short carrier diffusion length and hence a bilayer structure as shown in Fig. 2.10(a) is not effective for carrier collection, which necessitates the use of the more complicated nanostructured interface as shown in Fig. 2.10(b) [91]. Moreover, the high bandgap of $\mathrm{Cu}_{2} \mathrm{O}$ and $\mathrm{SnO}, 2.0 \mathrm{eV}$ and $2.7 \mathrm{eV}$ respectively, also leads to relatively low absorption, especially in the visible or IR range. Therefore, although all metal oxide based solar cells offer solution based processing as well as environmental stability, they still suffer from low performance with a PCE of around 4\% [91], attributed to the low diffusion length as well as the lack of absorption.

a)

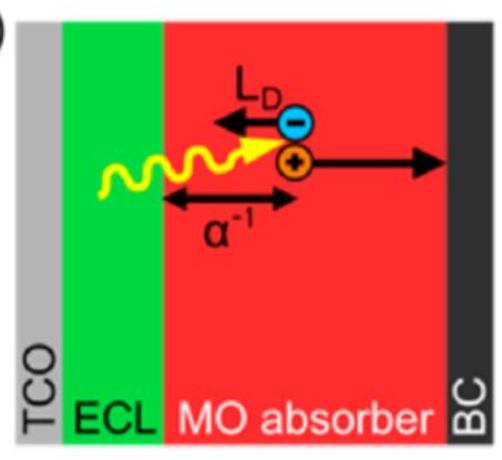

b)

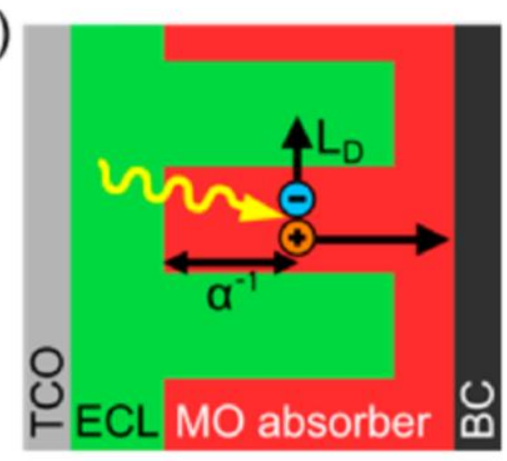

Figure 2.10 : All metal oxide based solar cell with (a) bilayer structure and (b) nanostructured interface [91]. 
In summary, conventional crystalline Si solar cell allows high performance device due to its good optoelectronic properties. However, the cost and energy requirement for its fabrication is high, due to high temperature diffusion process involved. Amorphous Si based solar cell allows low temperature process with PECVD deposition but has low throughput and low performance issues. In contrast, organic solar cell offers solution based processing that enables low temperature, high throughput process over a large area. However, it suffers from low performance due to the poor electrical transport in the organic material, as well as performance instability. On the other hand, metal oxide based solar cell offers solution based processing, performance stability, and high transparency selective carrier contact. However, it has the drawbacks that the metal oxide absorber layer exhibits poor electrical transport properties and a lack of optical absorption.

\subsection{Silicon heterojunction solar cell}

Although amorphous $\mathrm{Si}$, organic and metal oxide solar cells offer low temperature processing, all of them suffer from poor electrical transport properties in its absorber. This prompted the idea of developing Si heterojunction solar cell, where crystalline Si with good electrical transport property is used as the main light absorber, while the selective carrier contacts (SCCs) layer are formed using other materials deposited by low temperature process, thus replacing the high temperature $\mathrm{n}^{+}$or $\mathrm{p}^{+}$layer diffusion process. Essentially, we leverage on the good 
optoelectronic properties of crystalline $\mathrm{Si}$, as well as the low temperature processing advantage of other materials.

One of the most established and mature silicon heterojunction solar cells is based on crystalline $\mathrm{Si}$ absorber, in contact with $\mathrm{p}$-type and n-type amorphous $\mathrm{Si}: \mathrm{H}$ serving as hole and electron collecting SCCs, respectively. Intrinsic amorphous $\mathrm{Si}: \mathrm{H}$ is further deposited in between the crystalline $\mathrm{Si}$ and the doped amorphous Si:H layers to serve as a passivation layer. Such a design is known as the heterojunction with intrinsic thin film (HIT) structure, as illustrated in Fig. 2.11 [100]. HIT cell with interdigitated back contact recently has been reported with a very high power conversion efficiency of $26.6 \%$ [101]. The fabrication of the HIT solar cell is complicated since plasma enhanced chemical vapor deposition (PECVD) process and multilayers deposition requiring toxic gases are involved. Moreover, although the PECVD technique used for amorphous Si:H deposition is a low temperature process, it suffers from low throughput [85] which hinder its commercialization. Therefore, it is desirable to develop Si heterojunction solar cell with high performance yet at low temperature, with high throughput process, and over a large area. This idea motivates the pursue of Si heterojunction solar cell formed with organic and transition metal oxide selective carrier contact layer which requires single step deposition and less amount of toxic gases. 


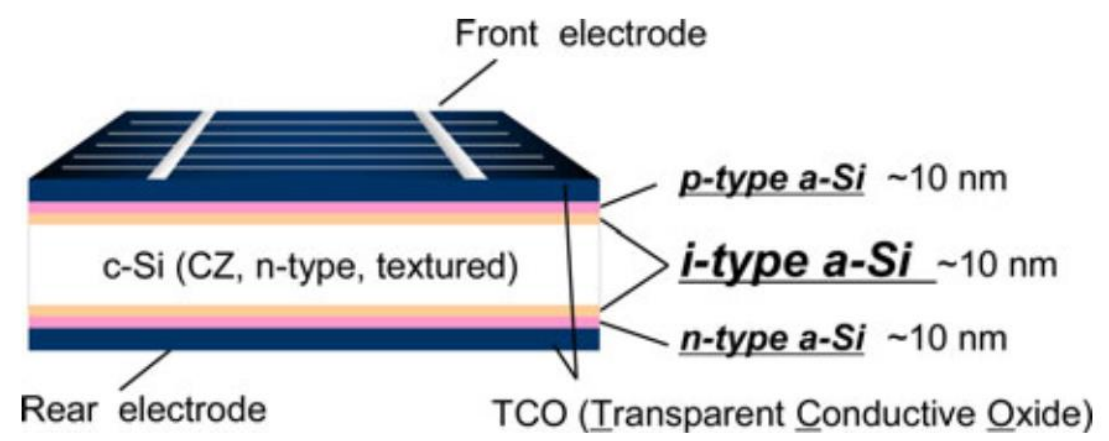

Figure 2.11 : Crystalline silicon heterojunction solar cell [100].

\subsubsection{Silicon / organic heterojunction solar cell}

A silicon / organic hybrid heterojunction solar cell offers high performance advantage of Si solar cell with solution based processing advantage of organic material [26-33]. In literature, the heterojunction solar cell is often referred to as silicon / organic hybrid (SOH) solar cell [31], with hybrid referring to the concurrent use of inorganic and organic materials in the structure. Figure 2.12 shows $\mathrm{SOH}$ solar cell with planar hybrid junction structure [102]. The n-type Si is used as the light absorber while the poly(3,4-ethylenedioxythiophene):polystyrenesulphonate (PEDOT:PSS) layer is used as the hole collector. Since PEDOT:PSS is almost transparent, most of the incident light is absorbed in the n-type $\mathrm{Si}$, generating electron and hole pairs. A significant number of the hole, which is the minority carrier, is diffused to the hybrid Si/PEDOT:PSS junction for collection. The holes in PEDOT:PSS will flow toward $\mathrm{Ag}$ grid, while the electrons in $\mathrm{Si}$ is collected on $\mathrm{Ti} / \mathrm{Pd} / \mathrm{Ag}$ contact. Its efficient collection is due to the relatively long minority carrier diffusion length in crystalline Si. He et al. has achieved a high efficiency of $11.3 \%$ 
[103] for the SOH solar cell based on a simple planar Si/PEDOT:PSS structure. Later, Jäckle et al. developed planar Si/PEDOT:PSS hybrid cell with 13.9\% [28] efficiency by optimizing Si substrate doping and with different PEDOT:PSS deposition process.

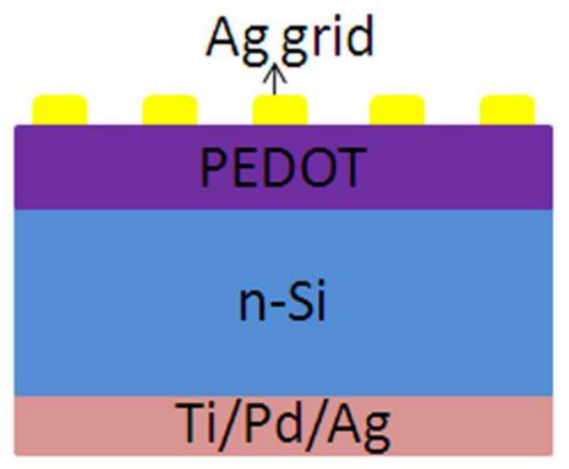

Figure 2.12 : Planar silicon / organic hybrid heterojunction solar cell [103].

Various organic materials have been used as selective carrier contact for $\mathrm{SOH}$ cells, mostly based on p-type organic polymer materials such as poly(3,4ethylenedioxythiophene) : polystyrenesulphonate (PEDOT:PSS) [26-33], Poly(3hexylthiophene-2,5-diyl) (P3HT) [104] and Poly(3-octylthiophene-2,5-diyl) (P3OT) [105] or small organic molecule such as 2,2',7,7'-Tetrakis[N,N-di(4methoxyphenyl)amino]-9,9'-spirobifluorene (Spiro-OMeTAD) [106] and 1,1-bis[(di4-tolylamino)phenyl]cyclohexane (TAPC) [34]. N-type polymers are rarely used since they are not as stable in ambient air as compared to $\mathrm{p}$-type polymers. Todate, most of the high performing $\mathrm{SOH}$ cells in the literature are based on the combination of n-type Si and PEDOT:PSS, due to the high transparency and high conductivity of PEDOT:PSS, and the excellent rectifying junction formed between $\mathrm{n}$-Si and this polymer. Although Si/PEDOT:PSS junction has been used extensively 
to realize high performing $\mathrm{SOH}$ solar cell, the nature of its junction, particularly whether it is a Schottky or p-n junction is still under debate [28-31, 39, 40]. As we shall present in Chapter 3, based on detailed reverse recovery transient measurements, we argue that the n-Si/PEDOT:PSS hybrid junction acts as an n$p^{+}$junction.

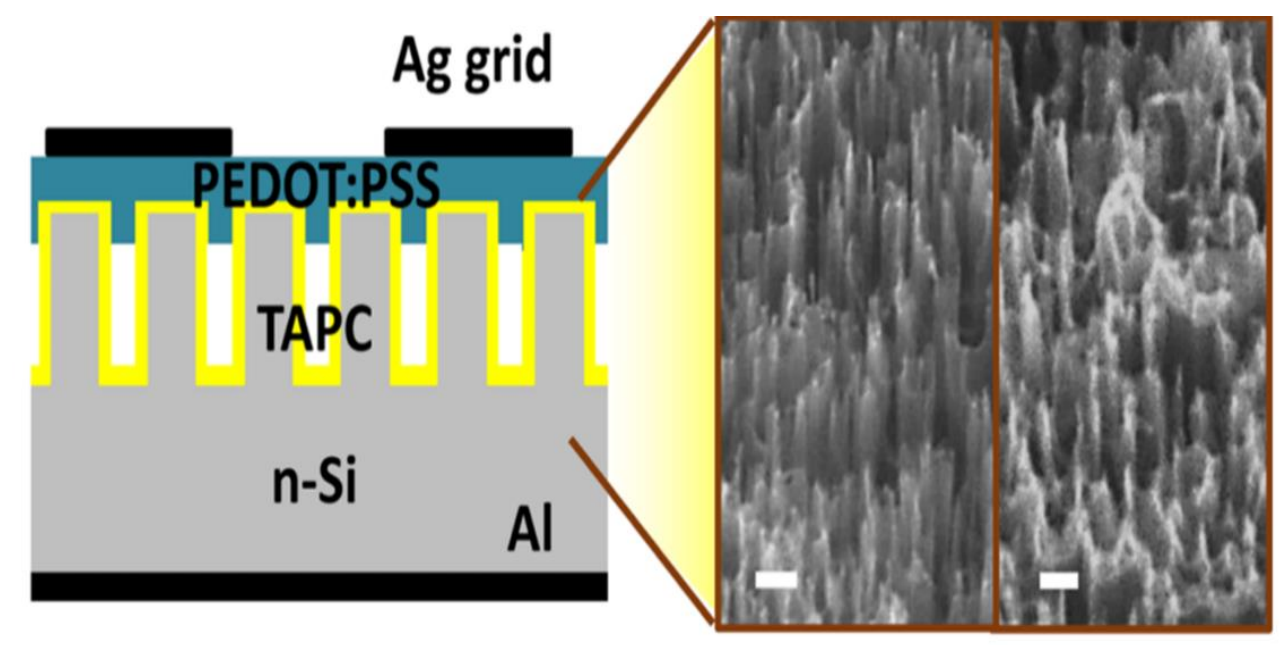

Figure 2.13 : Nanostructure interface silicon / organic hybrid solar cell [34]. The scale bar is $100 \mathrm{~nm}$.

While high efficiency may be achieved with planar $\mathrm{SOH}$ cell structure, it is noted that the cell suffers from a strong reflection of incident light, as PEDOT:PSS does not serve as an excellent antireflection coating in the structure. Nanostructures such as silicon nanowire [26], silicon nanocone [107] or silicon nanohole [108] arrays have therefore been introduced at the Si/PEDOT:PSS interface to reduce light reflectance. Figure 2.13 shows the cross section of a silicon nanowire / PEDOT:PSS hybrid solar cell with TAPC coated nanowire sidewall as developed by Yu et al. [34]. The silicon nanowires are produced from metal assisted chemical 
etching process. The PEDOT:PSS forms a layer on top of the silicon nanowires array while leaving air gaps between the nanowires wall, due to its large polymer molecules that do not penetrate well into the nanowire array. A layer of TAPC, a ptype small organic molecule, is coated on the silicon nanowires wall to provide passivation to the nanowire surfaces uncoated by PEDOT:PSS. The presence of TAPC coating layer also prevents excessive interfacial native oxide growth and provides enhanced performance stability [34].

Besides introducing nanostructures to minimize reflection of incident light, it is also important to reduce photocurrent losses by reducing the coverage of the top front grid electrode that results in shadowing losses. However, simply reducing the top grid coverage will also increase the series resistance losses due to the lateral transport of hole in the PEDOT:PSS layer. To address this issue, Chen et al. introduced silver nanowires (AgNWs) meshes on top of PEDOT:PSS [109] as seen in Fig. 2.14, which will help to receive hole from the PEDOT:PSS layer and transport it to the top grid. Due to a low sheet resistivity of the AgNWs meshes, longer distances between the top grid fingers can hence be tolerated. This has led to less shadowing loss and enhanced the PCE to $10.1 \%$, which is $36.5 \%$ higher as compared to the reference cell without the AgNWs meshes [109]. A high efficiency

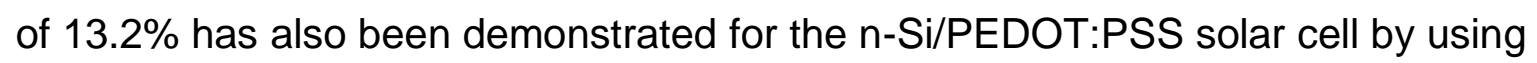
transfer imprinted gold meshes electrode [110]. 


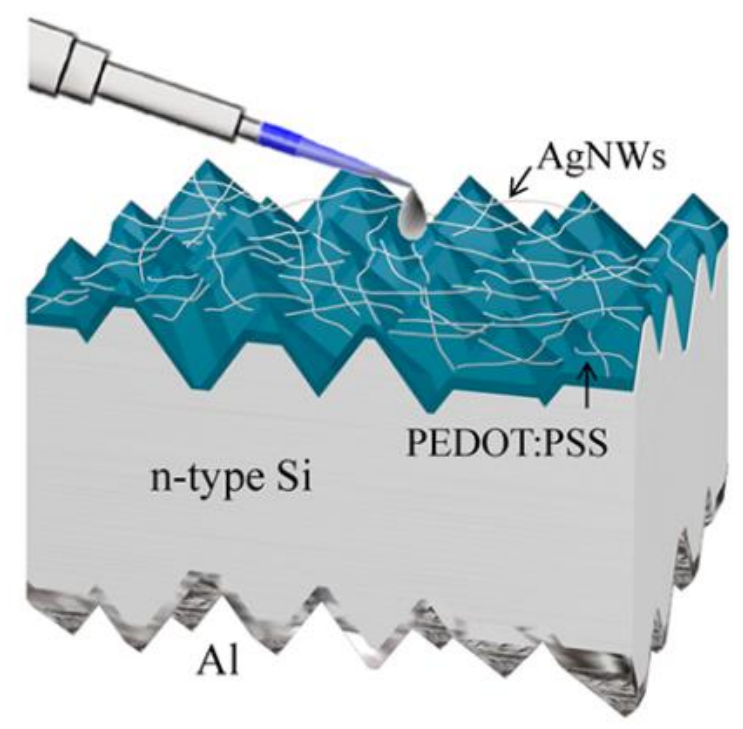

Figure 2.14 : Silicon / organic hybrid solar cell with silver nanowire meshes top electrode [109].

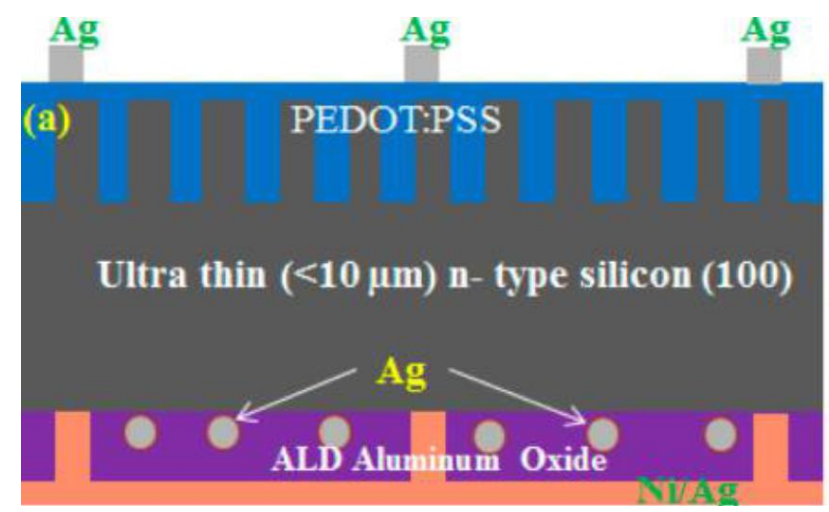

Figure 2.15 : Flexible ultra-thin $\mathrm{SOH}$ solar cell with front surface nanowire and back surface plasmonic Ag nanoparticles embedded in aluminum oxide [111].

To reduce the cost introduced by the use of bulk crystalline Si substrate, development of $\mathrm{SOH}$ cell based on thin film silicon has recently attracted an interests. Such structure offers not only low cost process and material but also 
allows mechanical flexibility that opens up new applications requiring flexible solar cell. However, as thin film $\mathrm{SOH}$ suffers from a lack of absorption depth, it necessitates the incorporation of light trapping features in the cell. Figure 2.15 shows thin film SOH solar cell that applies both front and back surface light trapping structures, as developed by Sharma et al. [111]. Due to the use of the thin substrate, the conventional random micro-pyramid texture is not a suitable choice for the antireflection purpose. Instead, a silicon nanowires array was used on the front surface to minimize light reflection. At the back side, a layer of aluminum oxide provided back surface passivation as well as refractive index difference that enhanced light reflection back into Si. Silver nanoparticles were also embedded inside the aluminum oxide to provide a plasmonic effect that improved light trapping inside the thin Si substrate [111]. However, only marginal improvement of $J_{s c}$ from 14.71 to $14.97 \mathrm{~mA} / \mathrm{cm}^{2}$ can be achieved, even for a thin $5.7 \mu \mathrm{m}$ thin Si substrate. The entire structure exhibited mechanical flexibility and the efficiency was maintained even after bending tests [111]. 


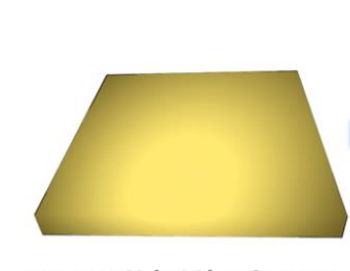

N type Si (100) substrate

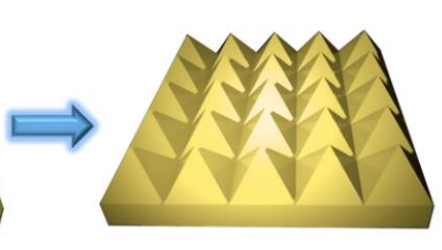

Pyramid Si by wet etching

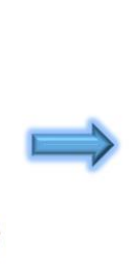

Preparing various concentrations of GQDs in PEDOT:PSS

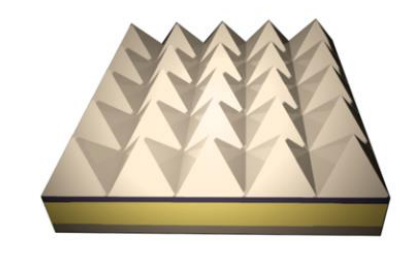

Deposition of the top and back electrodes

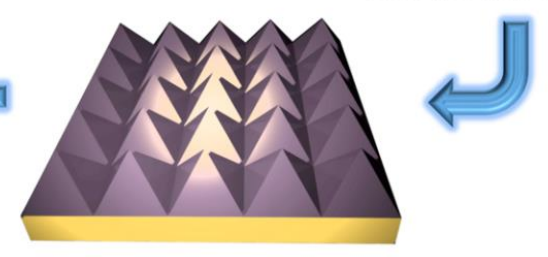

Spin-coating of PEDOT:PSS with GQDs

Figure 2.16 : Silicon / PEDOT:PSS hybrid solar cell with graphene quantum dots (GQDs) as photon down-converter [112].

Figure 2.16 shows Si/PEDOT:PSS hybrid solar cell that employs graphene quantum dots (GQDs) to enhance photocurrent. The GQDs are mixed with PEDOT:PSS solution at various concentrations, then spin coated on top of pyramid textured $\mathrm{Si}$. The GQDs act as a photon down-converter that converts high energy UV-photons absorbed into lower energy photons. Unlike a graphene sheet that has no bandgap, GQDs has a reduced sheet dimension that allows quantum confinement effect, producing size tunable bandgap. When light is absorbed in a GQD, an electron is excited from the ground state into a higher energy level corresponding to the absorbed photon. Since, the energy level is not stable, the electron drop into a lower energy level. Subsequently, the electron will recombine releasing low energy photons that can be absorbed slightly deeper into Si substrate [112]. The shift in the absorption depth allows electron-hole pairs to be generated away from the defective front surface, thus reducing recombination. The GQDs 
were observed to help improve the spectral response of the hybrid cell at short wavelength region as compared to that of the reference cell. In addition to the improved optical response, GQDs also enhanced electrical conduction inside the PEDOT:PSS, leading to reduced series resistance and improved fill factor. This combination of optical and electrical enhancement resulted in the increased $J_{s c}$ and FF of $36.26 \mathrm{~mA} / \mathrm{cm}^{2}$ and $63.87 \%$, as compared to those of the reference cell of $32.11 \mathrm{~mA} / \mathrm{cm}^{2}$ and $62.85 \%$, respectively [112]. Si/PEDOT:PSS hybrid solar cell with GQDs had been reported with $13.22 \%$ conversion efficiency [112].

Although PEDOT:PSS is mostly transparent, a small amount of light absorption still takes place inside the PEDOT:PSS layer. Due to the short exciton diffusion length inside the organic polymer, the carriers generated readily recombine and are not collected at the Si/PEDOT:PSS interface. Hence, light absorption inside PEDOT:PSS is considered as parasitic absorption since it does not contribute toward useful photocurrent. Zielke et al. [27] introduced the back PEDOT:PSS junction structure in which the PEDOT:PSS layer is located on the back surface of $\mathrm{Si}$, away from the incident light as shown in Fig. 2.17 in order to alleviate the parasitic absorption. Such a structure has been shown to result in a higher PCE of $17.4 \%$, as compared to that of front junction cell of around $12.3 \%$ fabricated in the same work [27]. Zhang et al. [41] have also recently reported a remarkable PCE of $16.1 \%$ for Si/PEDOT:PSS back junction cell, which is $33 \%$ higher as compared to that of front junction cell. Despite the promising results obtained for back junction cell, there are some drawbacks with such a device architecture. Due to the 
presence of the front metal electrodes, the front surface passivation is more demanding to ensure minimum carriers recombination. In addition, since the cell junction is situated away from the incident light, it imposes a stricter demand on the quality of Si to ensure high minority carrier lifetime, so that the carriers generated can be effectively collected. In view of the above, front junction cell structure is still attractive from the consideration of process simplicity and lower material cost. In Chapter 4, we will present a cell design based on the front junction structure that can effectively address the parasitic absorption problem, by employing a thin PEDOT:PSS layer in conjunction with an ARC compensated layer on top of it. We obtained a PCE for the proposed cell which is comparable to that of back junction cell. In addition, interdigitated back contact structure will also be proposed to reduce optical losses even further while allowing contact free front surface.

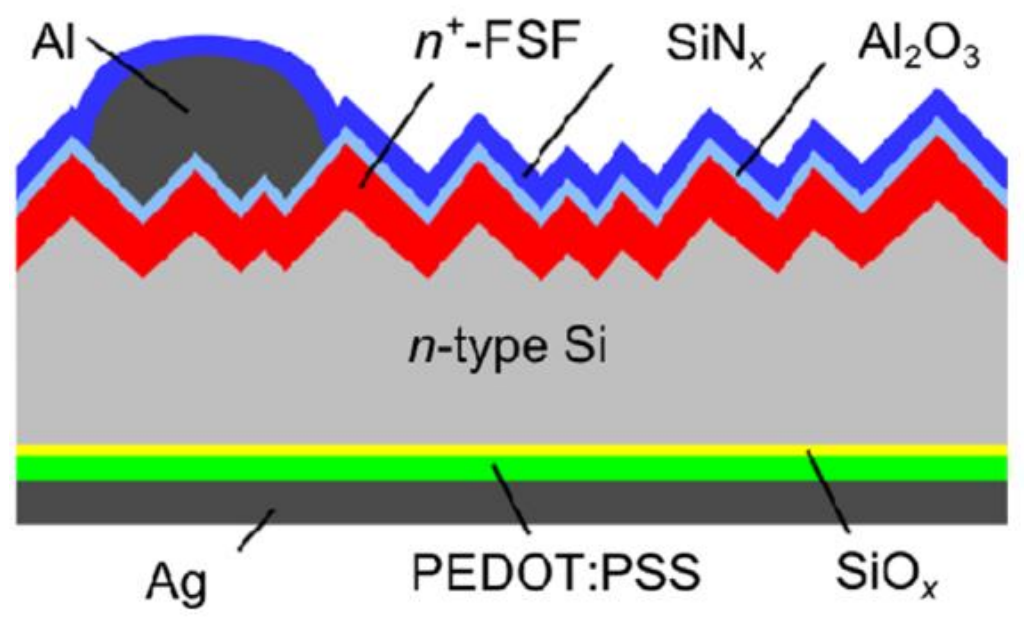

Figure 2.17 : Silicon / PEDOT:PSS hybrid solar cell with back junction structure [27]. 


\subsubsection{Silicon / transition metal oxide heterojunction solar cell}

Si heterojunction solar cell based on amorphous Si [113] or organic SCC [27] materials suffer from parasitic optical absorption losses. The parasitic absorption issue demands the use of sophisticated front surface antireflection feature or adoption of back junction structure. The use of SCC based on transition metal oxide (TMO) enables the adoption of simple front junction structure that does not suffer from parasitic absorption, owing to the high transparency and wide bandgap of the TMO. Figure 2.18 shows a schematic of silicon / transition metal oxide heterojunction solar cell with a planar interface formed between n-type c-Si and molybdenum oxide $\left(\mathrm{MoO}_{\mathrm{x}}\right)$ layer [54]. Such planar structure exhibits high $\mathrm{J}_{\mathrm{sc}}$ of 35 $\mathrm{mA} / \mathrm{cm}^{2}$ and PCE of $16.7 \%$. The MoOx layer acts as an electron blocking layer, while the ITO serves as an antireflection coating material that concurrently improves the lateral conductance. Poly-Si $\left(\mathrm{n}^{+}\right)$layer is deposited on the back surface as an electron collecting layer, and it also serves to provide a back surface field. 


\section{moly - poly}

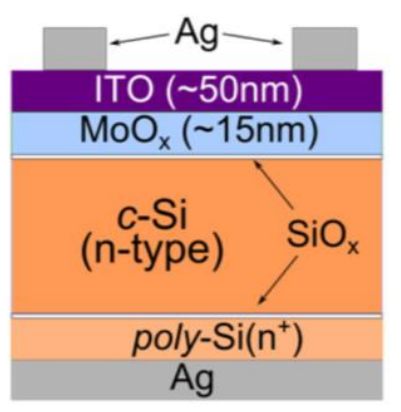

Figure 2.18 : Silicon / transition metal oxide heterojunction solar cell with planar heterojunction interface [54].

a)

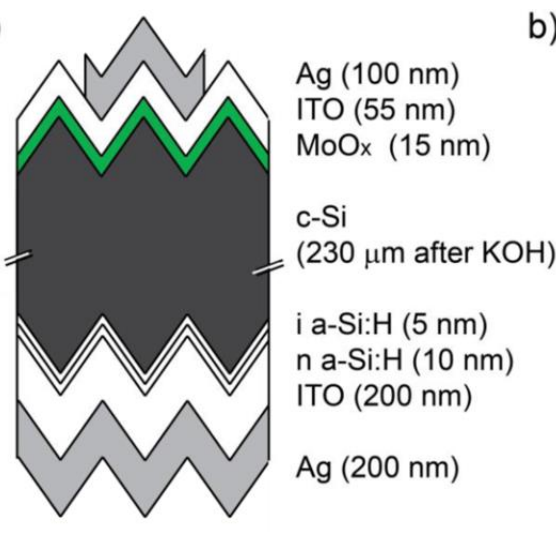

b)

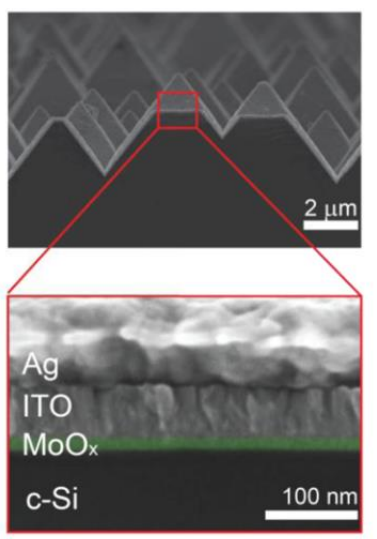

Figure 2.19 : Silicon / transition metal oxide heterojunction solar cell with textured heterojunction interface [97].

Higher $\mathrm{Jsc}_{\mathrm{sc}}$ of $38 \mathrm{~mA} / \mathrm{cm}^{2}$ has been achieved for $\mathrm{Si} / \mathrm{TMO}$ heterojunction solar cell by using textured Si surface as shown in Fig. 2.19 [97]. In this device, the antireflection coating effect of $\mathrm{MoO}_{x} / \mathrm{ITO}$ layer worked in synergy with the pyramid based texturing. As an electron collecting contact, several metal oxide materials such as titanium oxide $\left(\mathrm{TiO}_{2}\right)$, zinc oxide $(\mathrm{ZnO})$, aluminum-doped zinc oxide $(\mathrm{AZO})$ or fluorine-doped tin oxide (FTO) can be used to collect electron. For example, Fig. 
2.20 shows the use of $\mathrm{TiO}_{2}$ as an electron collecting contact at the back side of $n$ Si/PEDOT:PSS hybrid cell [95]. The solar cell device employs both Si/organic and $\mathrm{Si} / \mathrm{TMO}$ heterojunction as the hole and the electron selective contact respectively [95]. In principle, a Si/TMO heterojunction solar cell with both hole and electron selective contact can be made by using appropriate TMO for each layer, although at present, most work only report on single sided Si/TMO heterojunction.

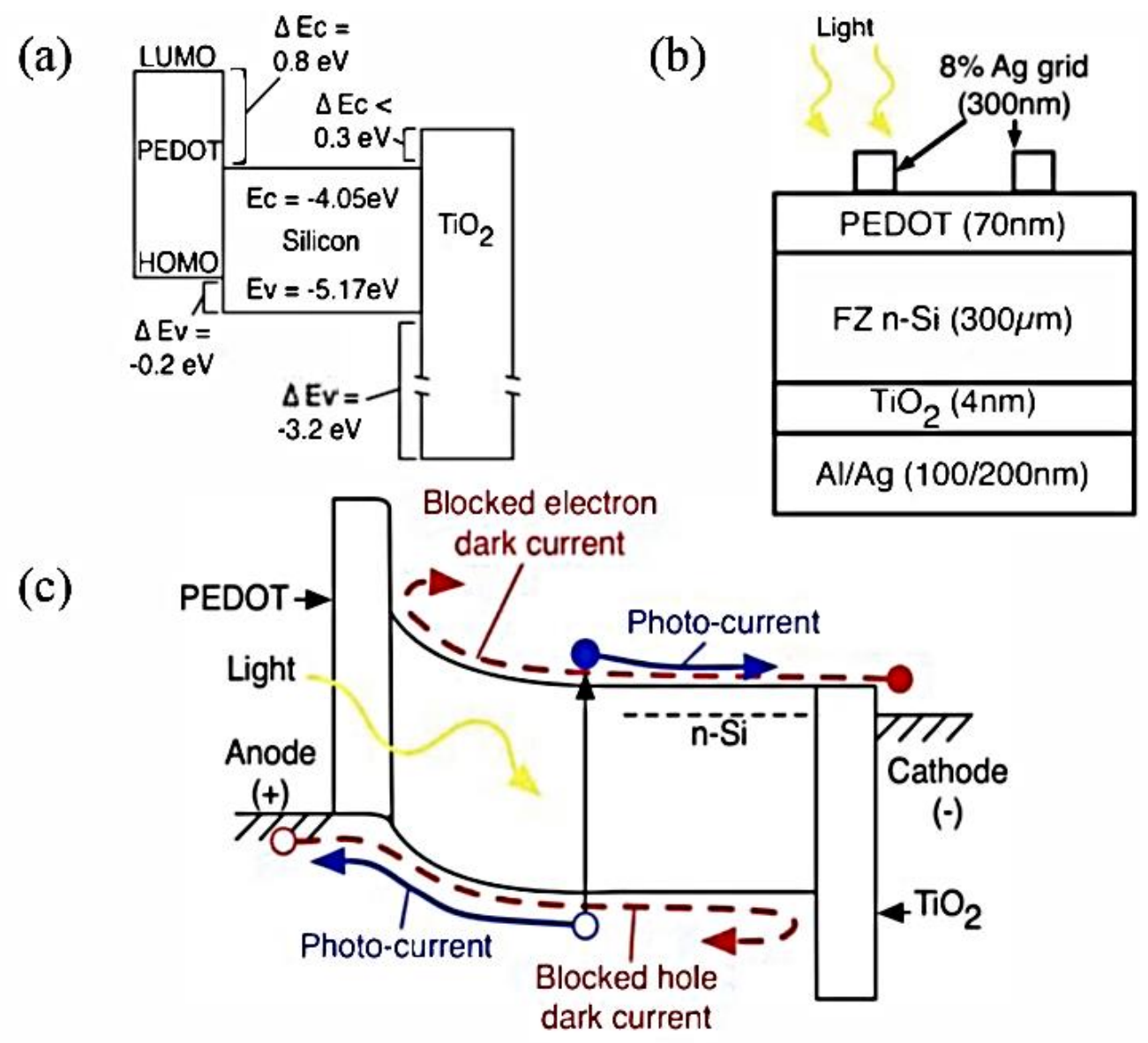

Figure 2.20: $\mathrm{TiO}_{2}$ as hole blocking TMO layer for n-type $\mathrm{Si}$ [95] (a) band structure, (b) device cross section and (c) operational principle. 
Currently, the study of $\mathrm{Si} / \mathrm{TMO}$ solar cells is still relatively new. There is a need to investigate its operation in terms of the band structure and alignment, interface properties and selective carrier extraction of the Si/TMO contacts. The use of TMO with Si light trapping nanostructure may be advantageous since it can develop conformal layer, providing a smoother gradient of refractive index as to Si/polymer structure. The use of silver nanowire meshes or alternative metal oxides such as AZO or FTO may help to reduce the cost by replacing the use of ITO. The use of doping on low conductivity TMO may be necessary to reduce its series resistance. The use of solution based processing of TMO on top of Si surface is also an interesting area of study to enable low temperature and high throughput fabrication process over a large area. In Chapter 5, we will present our work on planar Si/molybdenum oxide heterojunction solar cell, focusing on the optimization of the fabrication process of the cell using e-beam evaporation.

\subsection{Solar cell for small footprint application}

The growing interests on energy autonomous Internet of Things Smart Nodes (IoTSN) $[56,114-119]$ motivates the need for ambient energy harvesting devices that occupy small footprint at square centimeter $\left(\mathrm{cm}^{2}\right)$ or even square milliliter $\mathrm{mm}^{2}$ scale [55, 59-62]. Amongst the ambient energy available, photovoltaic based energy harvester is attractive [120], since it is an efficient approach and light energy is widely available under outdoor or indoor environment [59, 60, 120, 121]. However, IoT-SN often needs to use a solar cell with a footprint comparable or even larger than the circuit area [56, 59, 122]. Currently, there are growing interests 
in PV application in IoT-SN circuit, but they are mainly focused on energy conservation [63-66], layout specific arrangement $[58,59,61]$ or using exotic PV materials $[58,60,67]$ instead of boosting energy output per given footprint.

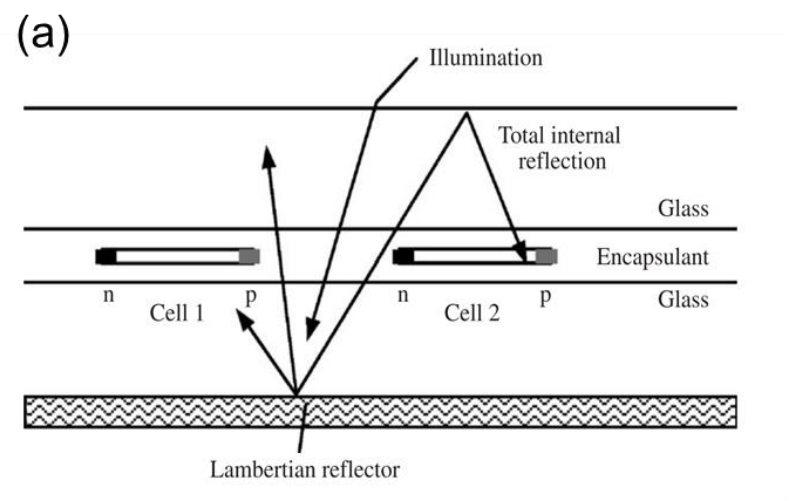

(b)

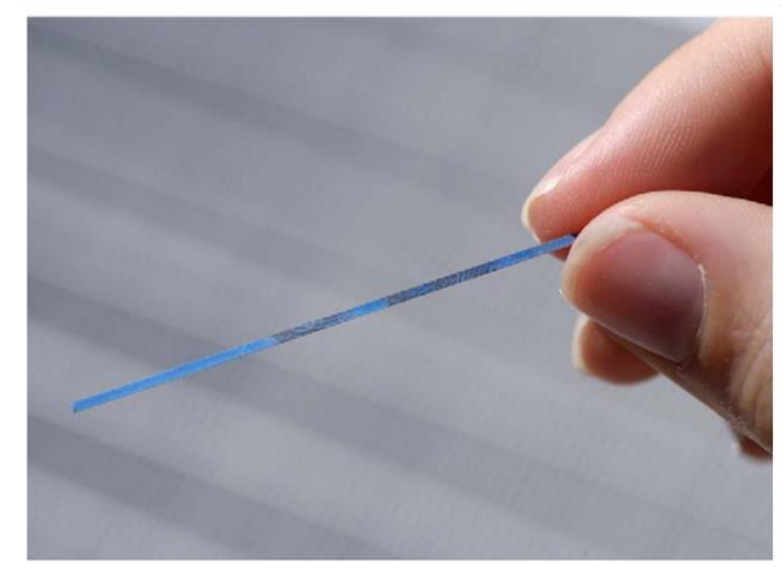

Figure 2.21 : (a) Cross section of Sliver ${ }^{\circledR}$ solar cell structure in which Lambertian reflector is used to increase the active surface area and (b) optical image of Sliver $^{\circledR}$ solar cell [123].

There are several methods that have been proposed that may increase energy output per given footprint. Verlinden et al. propose the use of Sliver ${ }^{\circledR}$ solar cell concept [33] in which solar cell is cut into several strips using micromachining, and 
then positioned horizontally at a particular distance and connected in large arrays. Fig. 2.21(a) shows the cross section of the Sliver ${ }^{\circledR}$ solar cell in which strips of silicon solar cell are positioned in a module apart from each other. Each individual strip acts as a bifacial structure, which can intercept light from the top and bottom. The gaps between the Sliver ${ }^{\circledR}$ solar strips allow incident light to reach the Lambertian reflector at the back to have multiple chances of reflection into the top or bottom part of the solar strips. This reduces the amount of Si material needed, without compromising on the amount of light absorbed. However, the lower packing density arrangement of the module increases its footprint. The above characteristics are in contrast to the PV-Tower cell concept that we proposed in Chapter 6 which can substantially improve the surface area for absorption by a factor of more than 100 yet over a very small foot print.

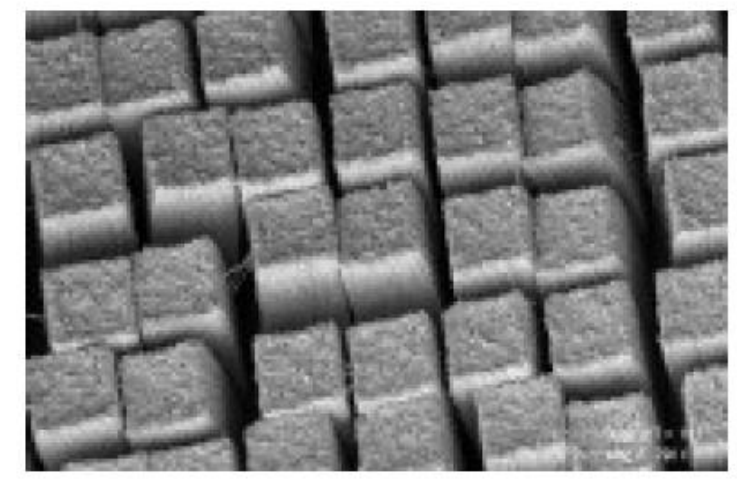

Figure 2.22 : SEM image of 3D solar cell structure $[124,125]$. The individual 3D solar cell structure has $40 \mu \mathrm{m} \times 40 \mu \mathrm{m}$ base and $100 \mu \mathrm{m}$ height.

Ready et al. $[124,125]$ proposed the use of 3D solar cell or "nano-manhattan" concept with an array of 3D towers in large number, closely packer over a large 
area. Figure 2.22 shows the SEM image of 3D solar cell concept. The 3D solar cell uses closely spaced towers to improve light trapping to increase the overall light absorption. Although it offers a significant increase in the power output over a given footprint, the increase is not proportional to the increased active surface area since it is hindered by mutual shadowing between the towers. The light trapping between the towers only intercepts secondary light reflected from the other towers instead of intercepting light directly from the ambient environment. A tower with a height of $100 \mu \mathrm{m}$ and base of $40 \mu \mathrm{m} \times 40 \mu \mathrm{m}$ only increases light absorption by a factor of 3 [124] as compared to a planar cell, although its surface area is increased by a factor of 11 . The concept has been further developed by Rampley et al. [126] that proposed methods to roughen the tower sidewall surface to increase random light scattering. Dagli et al. [127] also proposed a similar concept to 3D solar cell but with wide angle shaped pointed structure instead of rectangular tower, to promote multiple light reflections between the structures and increase light trapping as compared to the basic 3D solar cell as proposed by Ready et al. [124, 125]. 

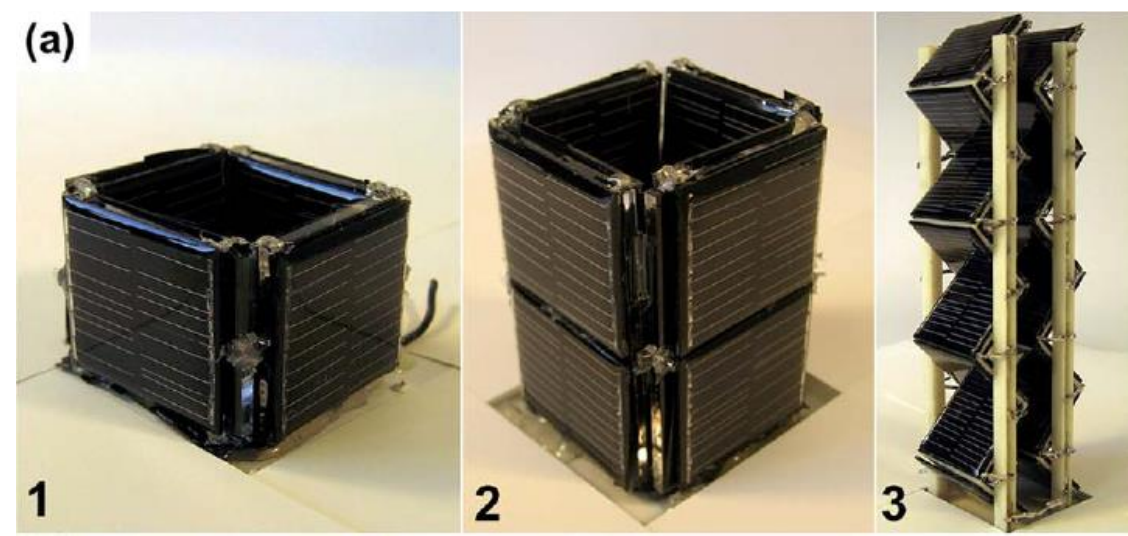

Figure 2.23: Three different stacks of multiple large area conventional PV modules in vertical structure [128].

Bernardi et al. [128] have proposed a different approach for increasing active light absorption area over a given footprint. Figure 2.23 illustrates the stacking of multiple conventional PV modules in a vertical structure that allows increased active surface area over a given footprint. In this case, ambient light can be harvested from the environment with minimum shadowing. However, this arrangement wastes a significant amount of costly semiconductor material [128] since it is merely a rearrangement of a number of conventional PV modules. This arrangement is also not replicable in a smaller $\mathrm{cm}^{2}$ or $\mathrm{mm}^{2}$ scale appropriate for miniaturized loT-SN. In Chapter 6, we will present a PV-Tower solar cell concept which allows high power generation over small footprint that is applicable for miniaturized loT-SN. 


\section{Chapter 3. Fabrication and characterization of planar n- Si/PEDOT:PSS hybrid inorganic/organic heterojunction}

In this chapter, planar hybrid inorganic/organic heterojunction formed between $\mathrm{n}$ $\mathrm{Si}$ and the conjugated polymer poly(3,4-ethylenedioxythiophene) : polystyrenesulphonate (PEDOT: PSS) is fabricated and characterized. To begin with, the motivation behind fabricating and characterizing the junction behavior of planar hybrid n-Si/PEDOT:PSS heterojunction will be presented. Subsequently, fabrication steps and the characterization technique applied will be introduced. Finally, the junction behavior of n-Si/PEDOT:PSS junction will be studied experimentally. Conclusion regarding the nature of n-Si/PEDOT:PSS junction behaviour will be used as an assumption for simulation and optimization work for various hybrid solar cells design in the next chapter.

\subsection{Introduction and motivation}

Si/organic hybrid heterojunction (SOH junction) formed between $\mathrm{n}-\mathrm{Si}$ and PEDOT:PSS exhibits a strong rectifying characteristic, and has attracted an of interest for application in various electronic devices [26-33, 39, 129, 130]. nSi/PEDOT:PSS hybrid junction is attractive as it can be fabricated using a simple solution-based process at low cost, low temperature $\left(\sim 100^{\circ} \mathrm{C}\right)$, and over a large area. The process simplicity presents an attractive alternative to conventional Si p$\mathrm{n}$ homojunction that requires high-temperature processing [131]. n-Si/PEDOT:PSS 
hybrid junction based solar cells have been extensively studied [26-33] with reported high efficiency of $17.4 \%$ [27]. This record efficiency has already approached the performance of typical commercial solar cell of $15-18 \%$ efficiency [15]. Flexible n-Si/PEDOT:PSS rectifying contacts have also been demonstrated based on flexible thin film Si [111]. Besides, hybrid junction formed between $\mathrm{Si}$ and PEDOT:PSS nanowire arrays have been used as a photodetector with tunable response [129]. n-Si/PEDOT:PSS junction has also been applied to form p-type layers for drain and source regions in a field effect transistor (FET) at low temperature [39] and to suppress base current in a bipolar transistor [130]. Improved device stability in the air has also been demonstrated for the junction [132-134], opening up the possibility of their practical use.

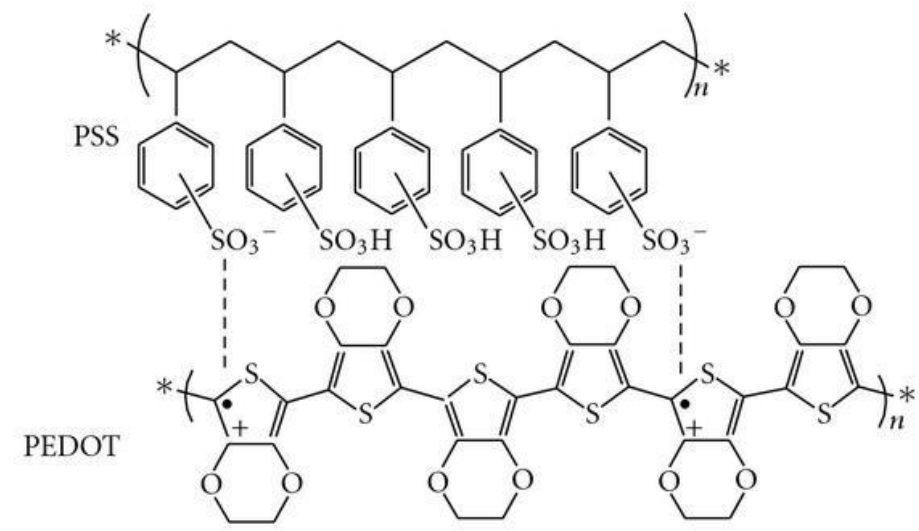

Figure 3.1 : Chemical structure of PEDOT:PSS [135]. Symbol "•" and "+" represent unpaired electron and positive charge.

PEDOT:PSS is a polyelectrolyte complex in which PEDOT acts as a hole conducting polymer that is attached to PSS as a host polyelectrolyte, as shown in 
Fig. 3.1. PEDOT itself is not soluble in any solvent, but with the introduction of water-soluble PSS, PEDOT:PSS can exist as stable water-based dispersion [136]. After deposited on top of $\mathrm{Si}$ with the solution-based coating process, PEDOT:PSS dispersion can be annealed to remove the water and form a gel-like film that can adhere to Si substrate, forming n-Si/PEDOT:PSS hybrid junction.

(a)
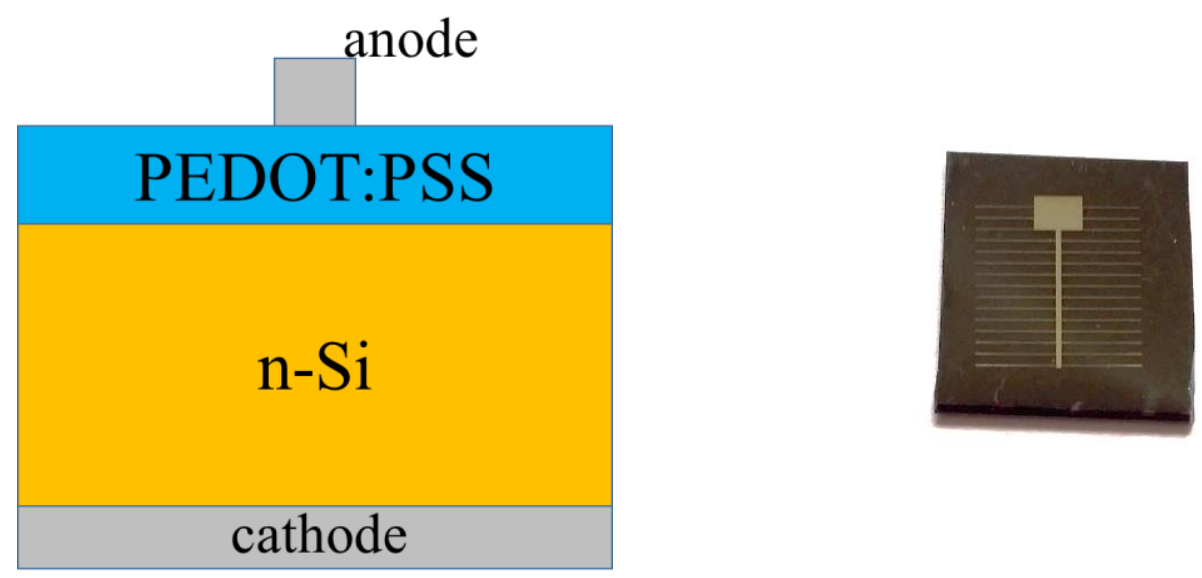

cathode

Figure 3.2 : (a) Scheme of n-Si/PEDOT:PSS hybrid junction structure and (b) optical image of n-Si/PEDOT:PSS hybrid solar cell.

Despite the ability of n-Si/PEDOT:PSS hybrid junction to form high-performance devices $[27,39,111,129,130]$, the nature of the junction has not been fully understood. Figure 3.2(a) shows the scheme of n-Si/PEDOT:PSS hybrid junction structure and its optical image is shown in Fig. 3.2(b). It is a subject of debate whether the SOH structure forms a p-n or Schottky junction [28-31, 39, 40], since PEDOT:PSS exhibits both semiconductor and metallic characteristics [35-38]. The 
dark saturation current $J_{0}$ will be dominated by diffusion of holes and trap-assisted recombination as seen in Fig. 3.3(a) if it is the former, and by thermionic emission of electrons as seen in Fig. 3.3(b) if it is the latter [78]. The understanding of the exact nature of this $\mathrm{SOH}$ junction is important for the modeling and optimization of devices formed using this structure. A fundamental study on the junction behavior of this hybrid organic/inorganic structure is also interesting in itself, due to the prevalent use of PEDOT:PSS as the organic material and $\mathrm{Si}$ as the inorganic semiconductor.

(a)

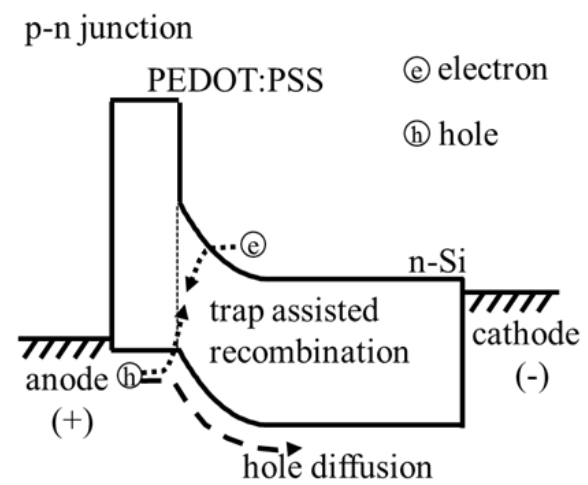

(b)

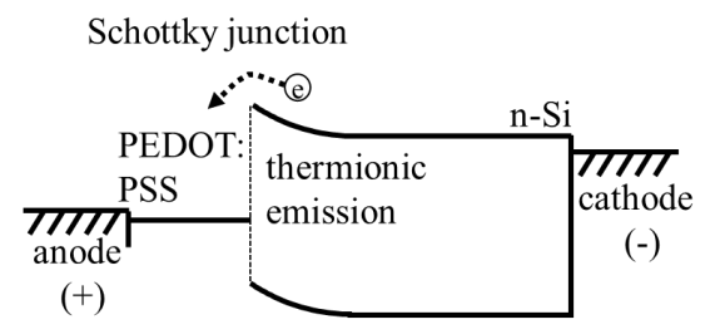

Figure 3.3 : n-Si/PEDOT:PSS structure based on (a) p-n and

(b) Schottky junction model.

There have been several studies on the n-Si/PEDOT:PSS hybrid junction, with conflicting conclusions that supported either the p-n or Schottky junction model. Lin et al. [137] found from C-V and dark I-V measurements that n-Si/PEDOT:PSS has a higher Schottky barrier and lower dark current as compared to n-Si/Au junction, leading to a high-quality Schottky junction, attributed to an interfacial dipole at the 
Si/organic interface. Shen et al. [29] increased the work function $\phi$ of PEDOT:PSS by subjecting it to an $\mathrm{N}_{2}$ ambient. They found from $\mathrm{C}-\mathrm{V}$ measurement that this had resulted in an increase in both the built-in potential $\left(V_{b}\right)$ and the solar cell open circuit voltage $\left(V_{o c}\right)$, consistent with the Schottky model. Zhu et al. [30] modified $\phi$ of PEDOT:PSS by adding dopant which resulted in increased solar cell fill factor (FF), and applied the Schottky model to extract $V_{b i}$ successfully. On the other hand, Price et al. [40] tested the Schottky model by extracting the thermionic injection velocity of electrons from $n-S i$ into PEDOT:PSS from the dark $I-V$ response. They found that the value was significantly lower than that of a Si/Au junction, which suggests that the Schottky model might not be appropriate. Erickson et al. [39] argued that the low thermionic injection was due to high internal Si band bending induced by high PEDOT:PSS work function and a lack of Fermi level pinning. The band bending induced p-type inversion layer just below the hybrid $n$ Si/PEDOT:PSS interface, forming p-n homojunction with the bulk n-Si. Jäckle et al. [28] corroborated the existence of p-type inversion layer but argued that the observed increase in the photovoltaic open circuit voltage $V_{o c}$ with increased $\mathrm{n}-\mathrm{Si}$ doping suggested diffusion dominated $J_{0}$, consistent with an $n-p^{+}$heterojunction formed between n-Si and PEDOT:PSS.

A reverse recovery transient (RRT) measurement is a powerful technique in distinguishing between Schottky and p-n junctions. It was first applied by Nagamatsu et al. [31] to study $\mathrm{SOH}$ solar cell in a single device and injection condition, where it was concluded that the hybrid junction exhibited excellent 
electron blocking capability and that the dark current was primarily due to hole injection from the PEDOT:PSS into Si. To date, the RRT characteristic of $n$ Si/PEDOT:PSS is still not widely investigated and well understood, in particular about the effect of n-Si substrate doping $N_{D}$ and forward current injection level $I_{F}$ that can provide an important insight into the nature of the hybrid junction. In this chapter, we investigate the reverse recovery transient (RRT) response of $n$ Si/PEDOT:PSS hybrid junction, as a function of the $n$-Si doping concentration and forward bias current injection level, to elucidate the characteristic of the $\mathrm{SOH}$ junction.

\subsection{Fabrication and characterization of planar n-Si/PEDOT:PSS hybrid heterojunction}

\subsubsection{Fabrication of planar n-Si/PEDOT:PSS hybrid}

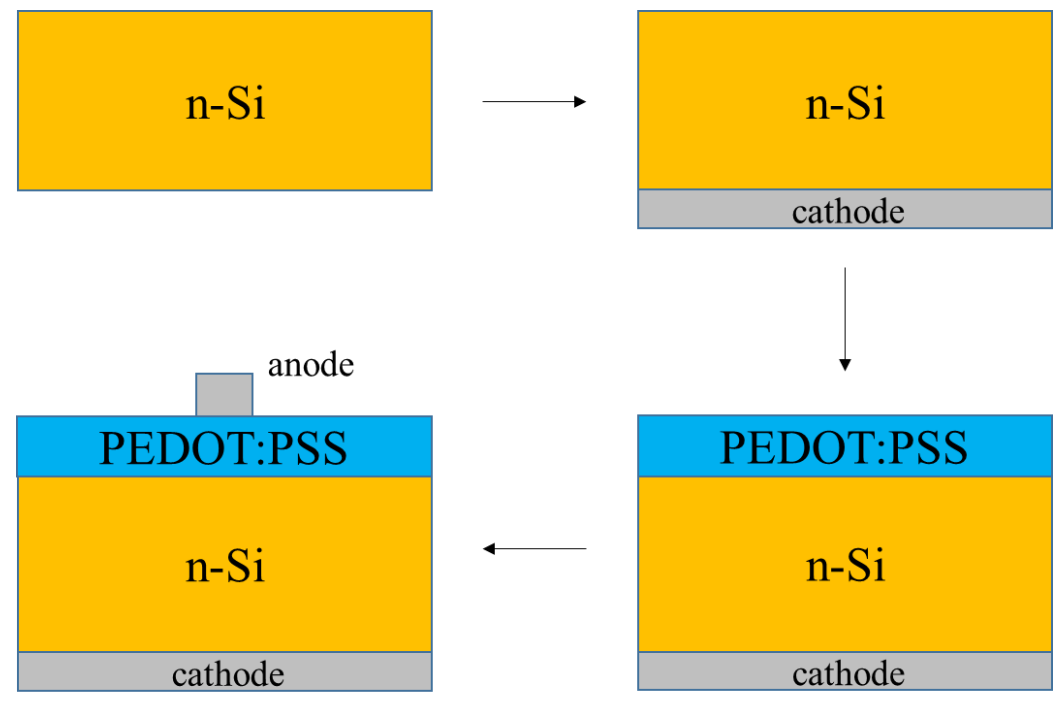

Figure 3.4 : Fabrication process of planar n-Si/PEDOT:PSS hybrid junction. 
Figure 3.4 shows the fabrication process of planar n-Si/PEDOT:PSS hybrid junction. The $\mathrm{n}-\mathrm{Si}<100>$ wafers were cleaned with acetone, IPA, and DI water sequentially, followed by immersion in $5 \% \mathrm{HF}$ solution to remove native oxide. A layer of $\mathrm{Ti} / \mathrm{Pd} / \mathrm{Ag}$ was deposited as the cathode on the back side of the $\mathrm{n}-\mathrm{Si}$ using e-beam evaporation. Clevios PH1000 variant of PEDOT:PSS solution was filtrated twice with $0.45 \mu \mathrm{m}$ PVDF filter before it was mixed with $5 \mathrm{wt} \%$ dimethyl sulfoxide (DMSO) to improve its conductivity and $1 \mathrm{wt} \%$ of Triton X-100 to improve its wettability to Si surface [102]. The PEDOT:PSS solution was dropped on hydrophobic Si surface and spin coated at $2000 \mathrm{rpm}$ for $60 \mathrm{~s}$, before annealed at $105^{\circ} \mathrm{C}$ for $10 \mathrm{~min}$ to remove the solvent and form n-Si/PEDOT:PSS junction. The thickness and the sheet resistivity of the PEDOT:PSS films were $70 \mathrm{~nm}$ and 100 $\Omega / s q$ respectively, as determined by ellipsometry and four-point probe measurements. Ag in the form of a metal grid was deposited as the anode on top of the PEDOT:PSS, which allows incident light to pass during photovoltaic characterization. The properties of Clevios PH1000 [138] is listed in Table 3.1.

Table 3.1: Properties of Clevios PH1000 [138].

\begin{tabular}{|l|c|c|}
\hline Properties & Values or description & Unit \\
\hline Solid content & $1.0-1.3$ & $\%$ \\
\hline Specific conductivity & 850 & $\mathrm{~S} / \mathrm{cm}$ \\
\hline Viscosity & $15-60$ & $\mathrm{mPas}$ \\
\hline Appearance & $\begin{array}{c}\text { Aqueous dispersion, } \\
\text { blue liquid }\end{array}$ & \\
\hline
\end{tabular}




\subsubsection{Characterization techniques}

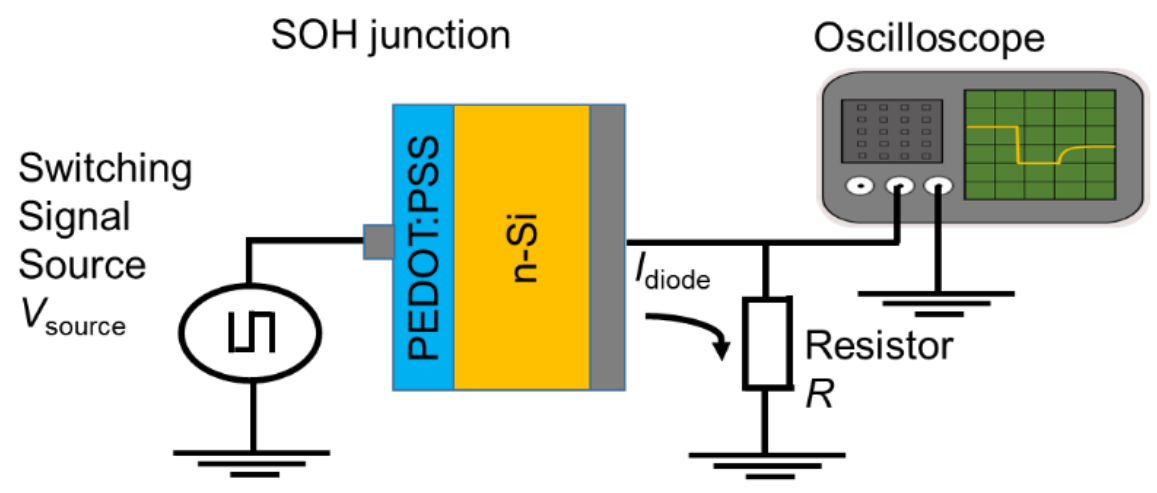

Figure 3.5 : Measurement setup for reverse recovery transient characterization of n-Si/PEDOT:PSS hybrid junction.

Figure 3.5 shows the measurement setup for the reverse recovery transient (RRT) characterization of n-Si/PEDOT:PSS hybrid junction. A switching voltage signal source is used to bias the n-Si/PEDOT:PSS hybrid junction in series with a resistor $R$. The switching voltage signal source will result in a diode current $l_{\text {diode, }}$ which is the output signal of the RRT characterization. The magnitude of /diode is monitored by measuring the voltage drop across the resistor $\mathrm{R}$.

Initially, the diode is forward biased to produce a steady state forward current $I_{F}$ and is then immediately switched to reverse bias. After switching, the diode will initially conduct a large reverse current $I_{R O}$ before it decays toward a steady state reverse current $I_{R S}$. For p-n junction, at the edge of the depletion region, there is an accumulation of excess minority carriers under forward bias as shown in Fig. 3.6(a) 
[78]. In order to reach a steady state reverse bias condition after the switching, a finite time period is required to gradually remove the minority carriers stored on both sides of the junctions, as shown in Fig. 3.6(b) [78]. Due to the stored minority carriers, $I_{R}$ will stay constant at $I_{R 0}$ for a period called the settling time $\tau_{S}$, which is the duration required for the removal of the stored minority carrier charge by recombination or reverse injection process [78].

(a)

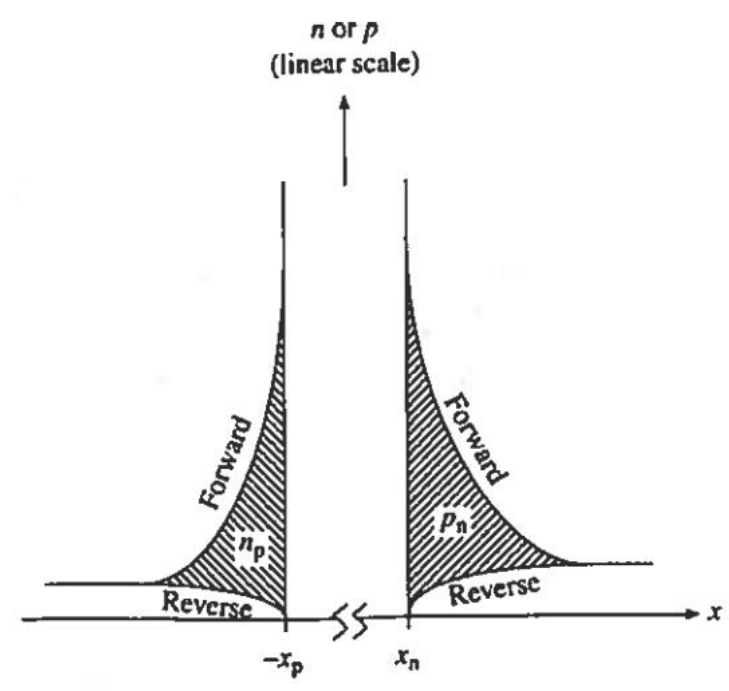

(b)

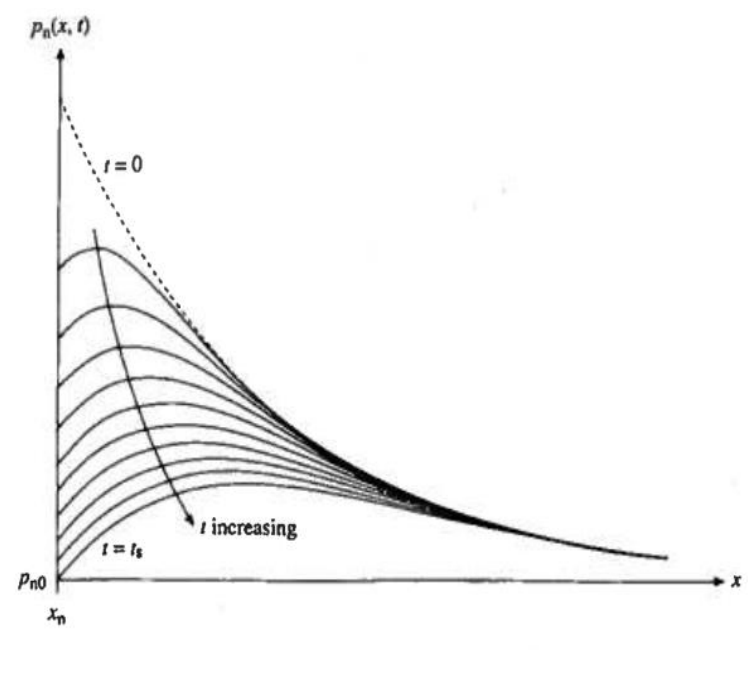

Figure 3.6 : (a) Theoretical minority carrier distribution profiles of $p-n$ junction under forward and reverse bias and (b) time-evolution of minority carrier distribution at $\mathrm{n}$-side after the reverse bias switching [78].

After minority carriers have been removed, the $p-n$ junction will experience an $\mathrm{RC}$ decay to reach steady state reverse current $I_{R S}$. The above desribed characteristic of a p-n junction is in contrast to that of Schottky junction. In the absence of minority 
carrier storage, the current will immediately experience RC decay and decrease toward $I_{R S}$ upon switching, and hence $\tau_{S} \approx 0$ [78]. Consequentially, the transient response of the reverse current $I_{R}$ for Schottky and $p$-n junctions will follow different decay curves as shown in Fig. 3.7 [78]. The distinct difference in the reverse recovery behavior of the Schottky and $p-n$ junctions provides a very useful means to distinguish between them. It should be noted that for a $\mathrm{p}^{+}-\mathrm{n}$ junction, the $\tau_{s}$ is related to minority carrier lifetime $\tau_{p}$ and $I_{R} d I_{F}$ by Eq(3.1) [78].

$$
\operatorname{erf}\left(\sqrt{\frac{\tau_{s}}{\tau_{p}}}\right)=\frac{1}{1+I_{R 0} / I_{F}}
$$

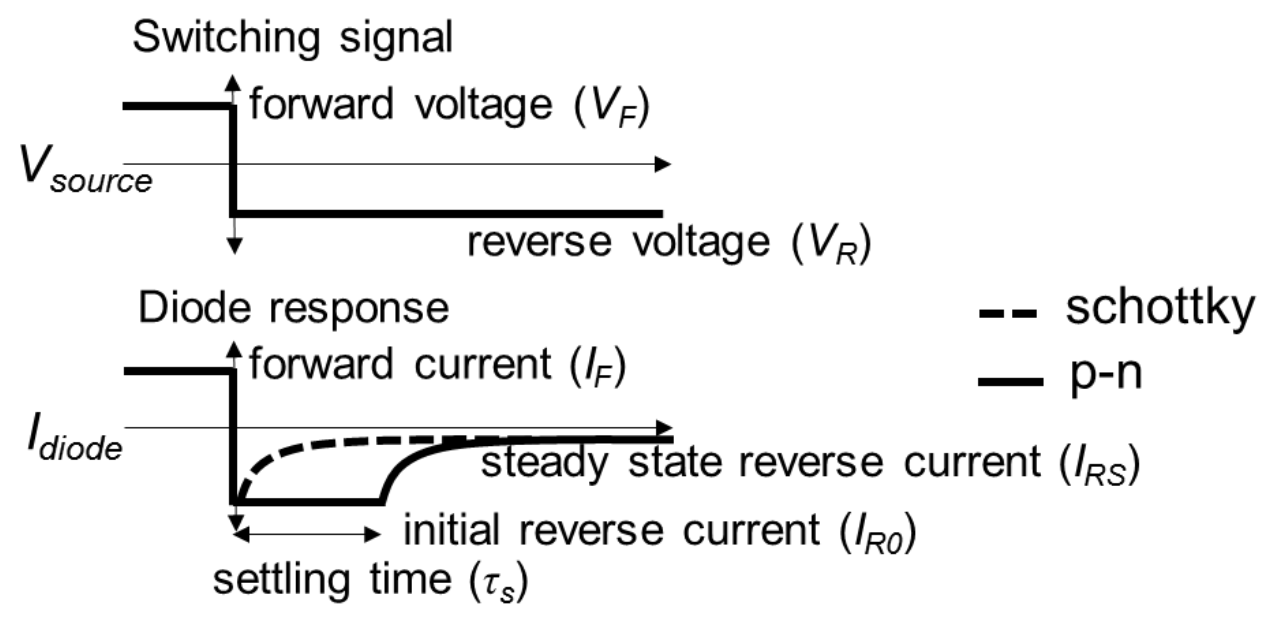

Figure 3.7 : Theoretical response of reverse recovery transient measurement for Schottky and p-n junctions.

We used the measurement setup as shown in Fig. 3.5 to perform the RRT characterization of the n-Si/PEDOT:PSS junction. A function generator was used to generate square wave switching signal at a frequency of $100 \mathrm{~Hz}$, and it applies 
a forward bias $V_{F}=+10 \mathrm{~V}$ at $\mathrm{t}<0$ and switches to reverse bias $V_{R}=-10 \mathrm{~V}$ at $\mathrm{t} \geq 0$. The diode current $I_{\text {diode }}$ was monitored by measuring the voltage drop across a 0.5 $\mathrm{k} \Omega$ resistor using an oscilloscope. The $\tau_{s}$ is determined as illustrated in Fig. 3.8, defined to be the intersection point between the maximum reverse current and the line tangent to a change threshold point. The change threshold point is defined as the point where the transient current has decayed to $90 \%$ of the difference between $I_{R O}$ and $I_{R S}$. The tangent line is drawn at this point by linear regression method using all of the data samples at $0.05 \mu$ s before and after the change threshold point. This approach will minimize the effect of the RC decay, and provide a more accurate measurement of $\tau_{S}$, instead of simply using the $90 \%$ of the difference between $I_{R O}$ and $I_{R S}$ as the reference point [139].

\section{Time}

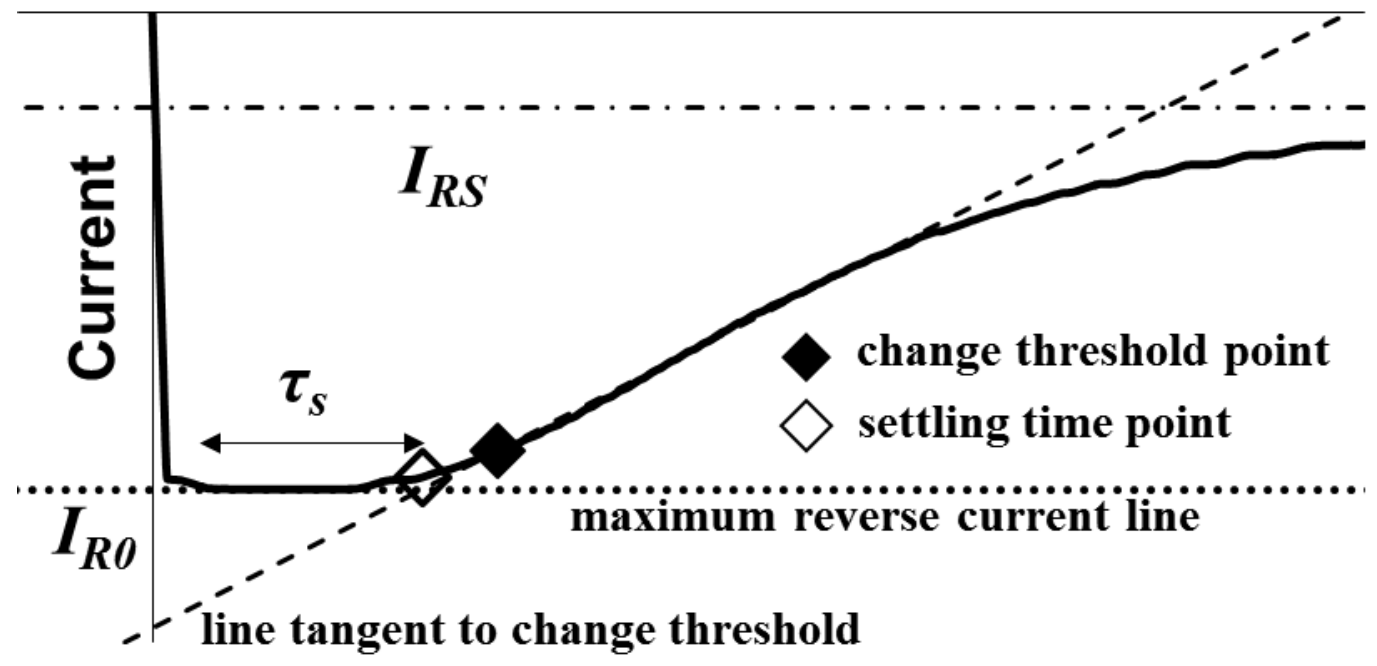

Figure 3.8 : Determination of settling time $\left(\tau_{s}\right)$ duration. 
The current density-voltage $(J-V)$ characteristics of the cells were examined using Keithley 2400 electrometer both under dark and illuminated conditions. A San-EI electric solar simulator with an air mass 1.5 (AM 1.5) filter at a power density of 100 $\mathrm{mW} / \mathrm{cm} 2$, was used to simulate standard light intensity for photovoltaic response measurement. A mask with an opening of $0.95 \mathrm{~cm}^{2}$ was used to prevent simulated light from falling into the outskirt region, to ensure an effective $0.95 \mathrm{~cm}^{2}$ cell area. The incident light intensity of the system was calibrated using a standard reference Si solar cell.

\subsection{Study of planar n-Si/PEDOT:PSS hybrid junction behavior}

\subsubsection{Reverse recovery transient characteristic}

The reverse recovery transient (RRT) response for samples with doping concentration $N_{D}=10^{14}$ to $10^{17} / \mathrm{cm}^{3}$ are shown in Fig. 3.9. It can be seen that there are two distinct decay modes for all the junctions studied, which is consistent with the p-n junction model depicted in Fig. 3.3(a). We observed $\tau_{s}$ of 23.5, 10.7, 10.0

and $8.3 \mu \mathrm{s}$ for the $\mathrm{SOH}$ junctions with $N_{D}$ of $10^{14}, 10^{15}, 10^{16}$ and $10^{17} / \mathrm{cm}^{3}$ respectively. The result obtained contradicts the Schottky junction model that predicts negligible settling time due to lack of minority carrier storage. 


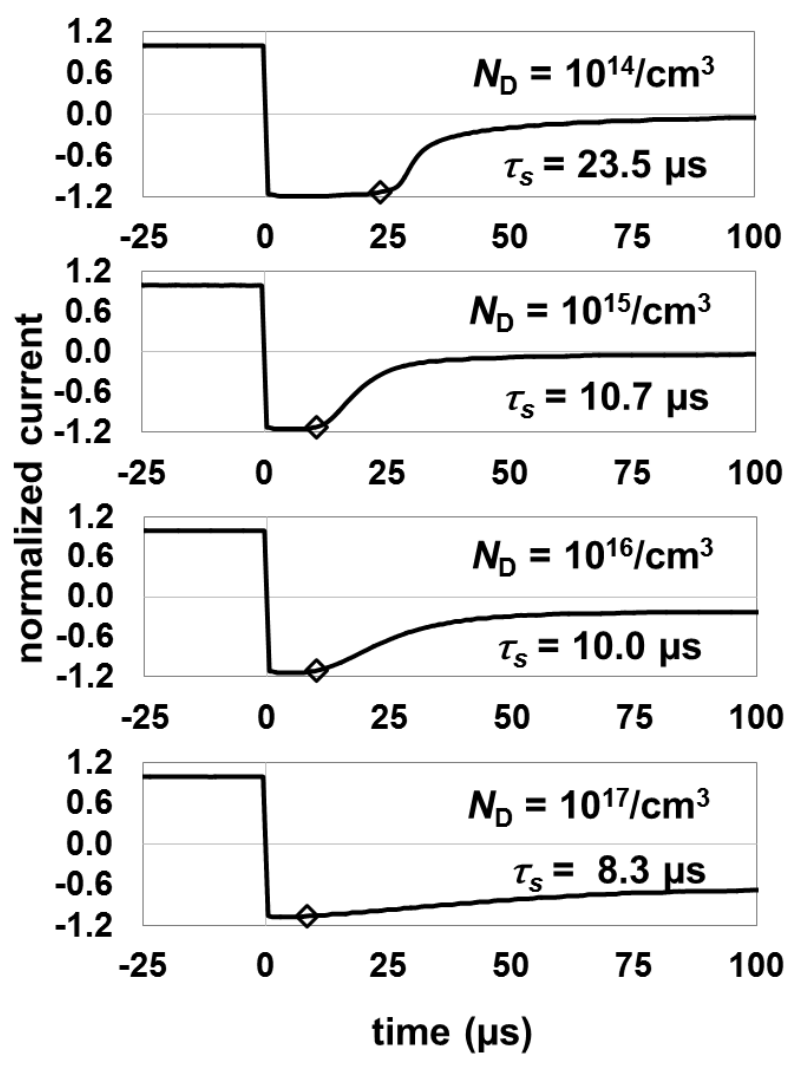

Figure 3.9 : RRT curves for Si/PEDOT:PSS hybrid junctions with different $N_{D}$. The current for each sample is normalized to $I_{F} \approx 18.1-19.0 \mathrm{~mA} / \mathrm{cm}^{2}$ and $I_{R O} / I_{F} \approx 1.1$ $-1.2$.

Using $\mathrm{Eq}(3.1)$, we can extract the $\tau_{p}$ from the measured $\tau_{s}$ and the results are shown in Table 3.2. Carrier lifetime $\tau_{p}$ of $126.8,55.5,51.4$ and $39.5 \mu$ s are deduced for $N_{D}$ of $10^{14}, 10^{15}, 10^{16}$ and $10^{17} / \mathrm{cm}^{3}$, respectively. The dependence of $\tau_{p}$ on $N_{D}$ is consistent with the model that the minority carrier lifetime is controlled by bulk recombination in the $\mathrm{n}$-Si. The bulk $\mathrm{n}$-Si controlled recombination can be explained by the asymmetric $n-p^{+}$junction formed, as the $p$-type PEDOT:PSS used in this study is expected to have a high level of hole carrier concentration. Electrochemical measurement shows the level of oxidation is about 1 charge per 3 EDOT monomer, 
while stoichiometric analysis determines EDOT monomer density of $10^{21} \mathrm{~cm}^{-3}$ for Clevios PH1000 formulation [136]. The hole concentration of $3 \times 10^{20} \mathrm{~cm}^{-3}$ can be deduced [136] by multiplying the level of oxidation $\approx 1 / 3$ to EDOT monomer density of $10^{21} \mathrm{~cm}^{-3}$. The high level of hole concentration allows $\mathrm{n}$-Si/PEDOT:PSS junction act as an $n-p^{+}$junction. Therefore the minority carriers will be mainly stored in the $\mathrm{n}$-Si side and not in PEDOT:PSS. The result is in contrast to the ZnO/PEDOT:PSS inorganic/organic hybrid junction [139], where the RRT deduced lifetime is attributed to the charge trapping process in PEDOT:PSS, represented by electron lifetime in PEDOT:PSS. The bulk $\mathrm{n}-\mathrm{Si}$ dominated carrier lifetime of $\mathrm{n}$ Si/PEDOT:PSS implies that devices built using the SOH junction can leverage on the good electronic properties of $\mathrm{Si}$, and is not limited by the poor electronic properties of the organic polymer.

Table 3.2 : Settling time $\tau_{s}$ and extracted carrier lifetimes $\tau_{p}$ for the hybrid junctions with different $N_{D}$.

\begin{tabular}{|c|c|c|}
\hline$N_{D}$ & $\tau_{s}$ & $\tau_{p}$ \\
$\left(/ \mathrm{cm}^{3}\right)$ & $(\mu \mathrm{s})$ & $(\mu \mathrm{s})$ \\
\hline $10^{14}$ & 23.5 & 126.8 \\
\hline $10^{15}$ & 10.7 & 55.5 \\
\hline $10^{16}$ & 10.0 & 51.4 \\
\hline $10^{17}$ & 8.3 & 39.5 \\
\hline
\end{tabular}


To further understand the result, we studied the RRT response of the $\mathrm{SOH}$ junction with $N_{D}=10^{16} / \mathrm{cm}^{3}$ as a function of $I_{F}$ at a constant $I_{R o} I_{F} \approx 1$, by maintaining a constant $V_{R O} / V_{F}$ ratio. The results are shown in Fig 3.10. Based on the charge control relationship used to derive $\mathrm{Eq}(3.1), \tau_{p}$ is a constant at a given doping concentration, while $\tau_{S}$ depends on $\tau_{p}$ and $I_{R o} / I_{F}$ ratio [78]. Hence we expect a minor change in $\tau_{S}$ if we change $I_{F}$ while keeping the $I_{R d} I_{F}$ ratio approximately constant. Nevertheless, it is observed that $\tau_{s}$ increases linearly with $I_{F}$ from 5.1 to 10.6 $\mathrm{mA} / \mathrm{cm}^{2}$, and nearly saturates at higher $I_{F}$. At low $I_{F}, \tau_{s}$ is very short compared to the RC decay time so that the recovery curve qualitatively appears similar to that of a Schottky junction.

From the measured $\tau_{s}$ and $I_{R O} / I_{F} v a l u e s$, we deduce $\tau_{p}$ using Eq(3.1), and the results are plotted in Fig. 3.11. It is evident from Fig. 3.11 that $\tau_{p}$ is proportional to $I_{F}$ for $I_{F}$ $<10 \mathrm{~mA} / \mathrm{cm}^{2}$. The dependence of $\tau_{p}$ on $I_{F}$ injection level cannot be explained by high injection level effect on bulk lifetime since it will shorten, rather than prolong the lifetime [78]. Instead, the dependence can be explained by the trap saturation effect at recombination centers at the hybrid interface. At lower $I_{F}$, these recombination centers dominate as compared to the bulk recombination process, resulting in a low $\tau_{s}$ and $\tau_{p}$. However, at higher $I_{F}$ when the recombination centers are saturated and do not participate in further trapping process, the measured $\tau_{p}$ will reveal the bulk Si lifetime, which should remain nearly constant and will be independent of the $I_{F}$. 


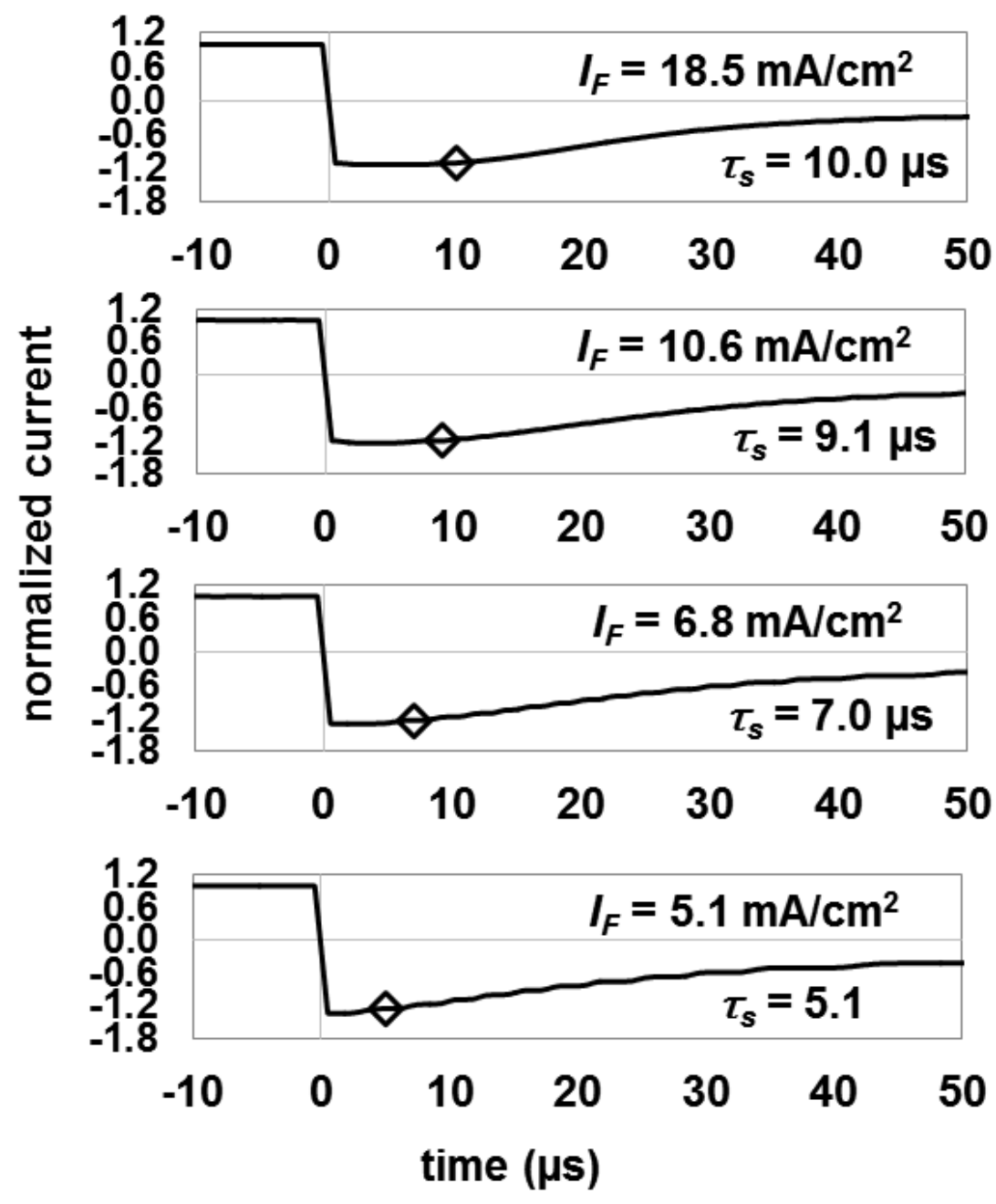

Figure 3.10 : RRT curves for the hybrid junction with $N_{D}=10^{16} / \mathrm{cm}^{3}$ at different forward bias injection levels $\left(I_{F}\right)$. The diode current is normalized to $I_{F}$. The settling times deduced are indicated by the diamond $(\diamond)$ marks.

This effect is indeed seen in our results shown in Fig. 3.11. The trap saturation effect was reported before for Si-based p-n homojunction [140-143]. Note that the $\tau_{p}$ values that are shown in Table 3.2 are already trap saturated since they were deduced at $I_{F}$ of $18.1-19.0 \mathrm{~mA} / \mathrm{cm}^{2}$. 


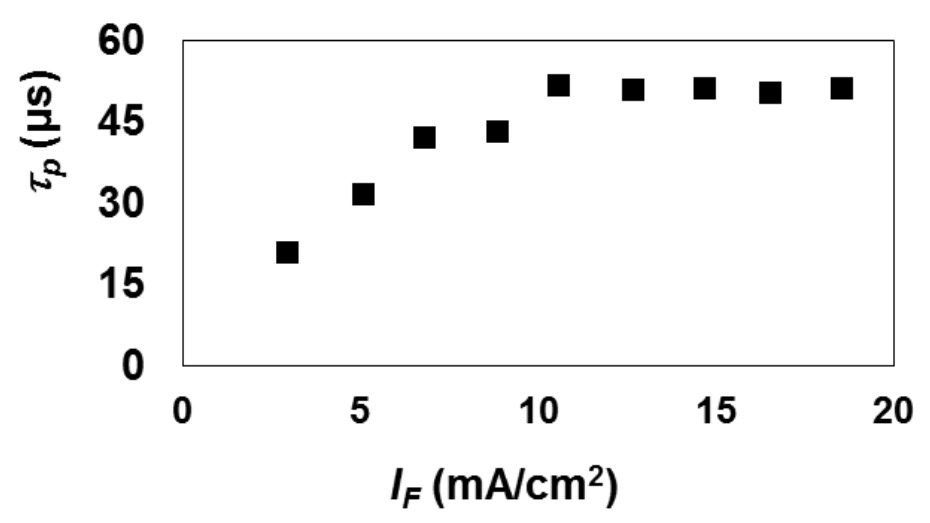

Figure 3.11 : Lifetime $\tau_{p}$ dependence on injection level $I_{F}$, deduced for the hybrid junction with $N_{D}$ of $10^{16} / \mathrm{cm}^{3}$.

\subsubsection{Dark current density-voltage characteristic}

We also measured the dark current density-voltage $(J-V)$ characteristics of the $n$ Si/PEDOT:PSS hybrid junctions, as shown in Fig. 3.12, to corroborate the junction characteristic deduced from the RRT measurements. Two slopes are observed in the $\ln ([J])-V$ curves, which is believed to be due to trap-assisted and diffusion current. We modeled the n-Si/PEDOT:PSS hybrid junction accordingly using the two-diode model as shown in Fig. 3.13. The $\mathrm{J}-\mathrm{V}$ curves of two diode models are described in $\mathrm{Eq}(3.2)$,

$$
J=J_{\text {diff }}\left(\exp \left(\frac{q\left(V-R_{S} J\right)}{n_{1} k T}\right)-1\right)+J_{T}\left(\exp \left(\frac{q\left(V-R_{S} J\right)}{n_{2} k T}\right)-1\right)+\frac{V-R_{S} J}{R_{S h}}
$$

where $R_{s}$ and $R_{s h}$ denote the series and shunt resistances, respectively, Jdiff and $n_{1}$ are the diffusion current and its associated ideality factor, while $J_{T}$ and $n_{2}$ are the 
trap assisted current and its associated ideality factor. Jdiff for the $p^{+}-n$ junction is described by $\mathrm{Eq}(3.3)$,

$$
J_{d i f f}=q \sqrt{\frac{D_{p}}{\tau_{p}}} \frac{n_{i}^{2}}{N_{D}}
$$

where $q$ is the electronic charge, $D_{p}$ is the diffusion constant, and $n_{i}$ is the intrinsic carrier concentration of Si. We can estimate $J_{\text {diff }}$ for the hybrid junctions, using the experimentally deduced trap-saturated $\tau_{p}$ for junctions with different $N_{D}$, as shown in Table 3.2, and with $D_{p}$ obtained from the literature [144]. The Jdiff obtained are shown in Table 3.3. We fitted $\mathrm{Eq}(3.2)$ to the experimental dark $J-V$ curves, incorporating the $J_{\text {diff }}$ deduced in Table 3.3, and based on an algorithm where $n_{1}$ is fixed at 1 [145], while the other parameters are allowed to vary.

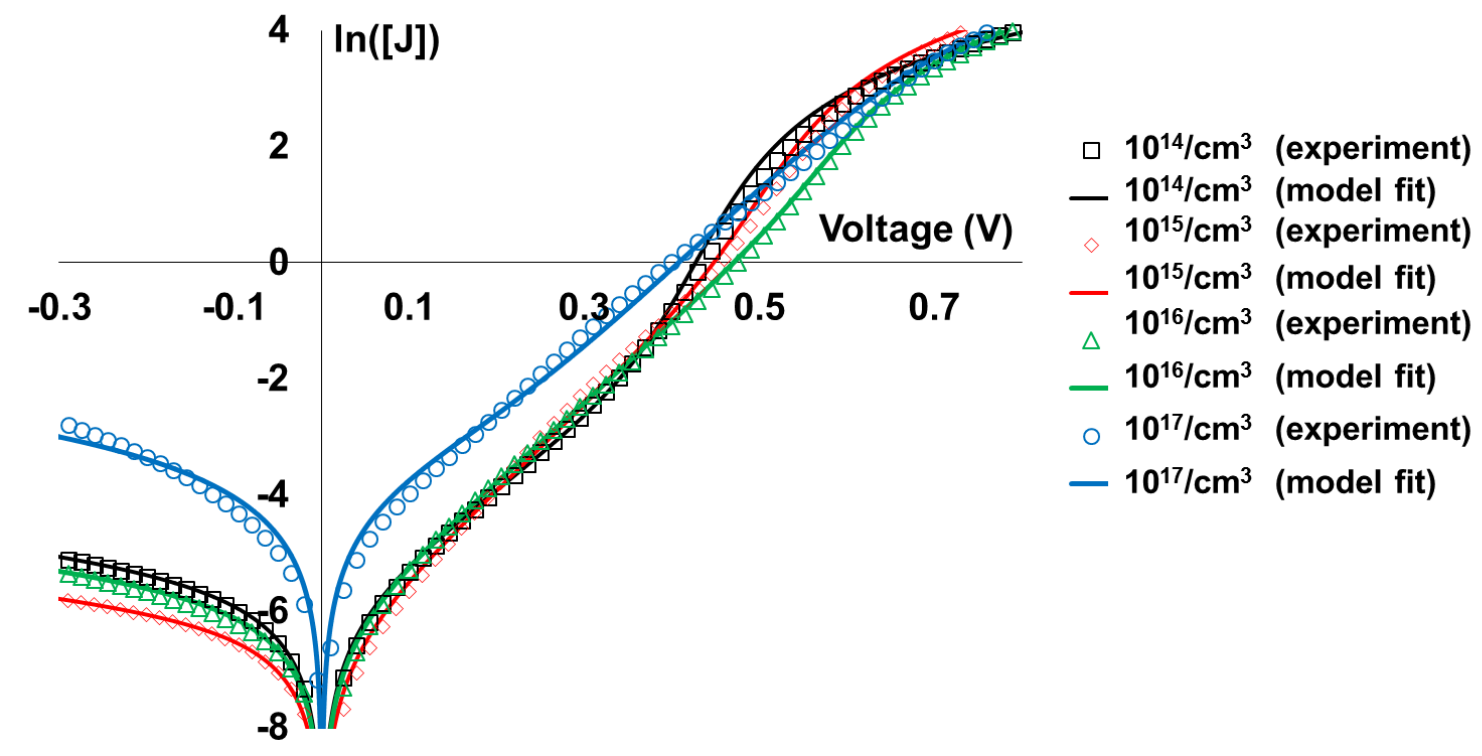

Figure 3.12: Dark $J-V$ response of planar n-Si/PEDOT:PSS junction at different $\mathrm{Si}$ substrate doping concentrations. 


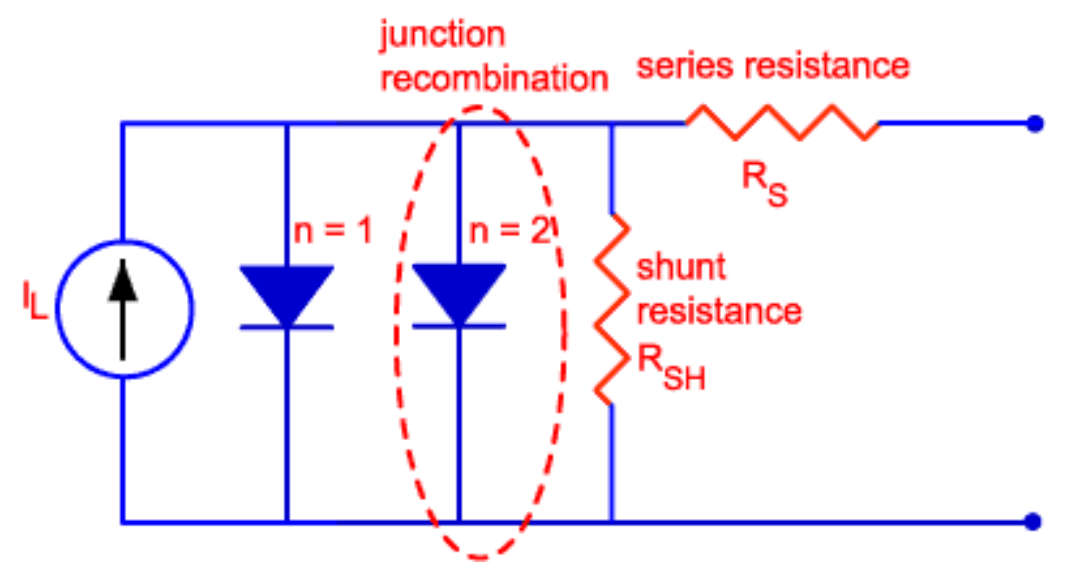

Figure 3.13 : Equivalent circuit of a two-diode model of a p-n junction [68].

Table 3.3 : Two-diode model parameters used to fit dark $J-V$ characteristic for the hybrid junctions with different $N_{D}$.

\begin{tabular}{|c|c|c|c|c|}
\hline \multirow{2}{*}{} & \multicolumn{4}{|c|}{$N_{D}\left(/ \mathrm{cm}^{3}\right)$} \\
\cline { 2 - 5 } & $10^{14}$ & $10^{15}$ & $10^{16}$ & $10^{17}$ \\
\hline$J_{\text {diff }}\left(\mathrm{A} / \mathrm{cm}^{2}\right)$ & $5 \times 10^{-11}$ & $7 \times 10^{-12}$ & $7 \times 10^{-13}$ & $7 \times 10^{-14}$ \\
\hline$J_{T}\left(\mathrm{~A} / \mathrm{cm}^{2}\right)$ & $1 \times 10^{-6}$ & $1 \times 10^{-6}$ & $1 \times 10^{-6}$ & $2 \times 10^{-6}$ \\
\hline$n_{2}$ & 3.1 & 2.6 & 2.8 & 2.6 \\
\hline$R_{S h}$ & 65.0 & 152.0 & 86.8 & 6.6 \\
$\left(\mathrm{k} \Omega . \mathrm{cm}^{2}\right)$ & & & & \\
\hline$R_{S}\left(\Omega . \mathrm{cm}^{2}\right)$ & 5.3 & 2.9 & 2.8 & 1.9 \\
\hline
\end{tabular}

The fitted parameters are presented in Table 3.3, and the fitted curves are shown in Fig. 3.12, where a good match is seen between the experimental and theoretical results for $N_{D}=10^{14}-10^{16} / \mathrm{cm}^{3}$. We notice that the ideality factor $n_{2}$ of the trap assisted current is greater than 2 for all the hybrid junctions, as expected of current 
that is associated with the trap-assisted recombination [146]. We also observe that $J_{T}>J_{\text {diff, }}$ which implies that the dark current at lower forward and reverse bias is dominated by the trap-assisted recombination current, while at large forward bias is dominated by the diffusion current. For $N_{D}=10^{17} / \mathrm{cm}^{3}$, the dark current does not fit the theoretical two-diode characteristic well. Instead, a more ohmic-like current is noted, as can also be observed from the lower $R_{s h}$ of this junction seen in Table 3.3. Previously, Walter et al. [132] studied the dark $I-V$ curves of $n$-Si/PEDOT:PSS junction formed with $\mathrm{n}-\mathrm{Si}$ and $\mathrm{n}^{+}-\mathrm{Si}$ and found that the junction behaviors were strongly rectifying and ohmic, respectively. The transition between strongly rectifying junction at $N_{D}=10^{16} / \mathrm{cm}^{3}$ to the almost perfect ohmic junction at $N_{D}=$ $10^{19} / \mathrm{cm}^{3}$ was attributed to an electron tunneling current [132] from Si conduction band to unoccupied electronic states at the bandgap of PEDOT:PSS, across a thinner depletion width due to higher $N_{D}$. This report is qualitatively in agreement with our observation in which slightly ohmic behavior is observed at $N_{D}=10^{17} / \mathrm{cm}^{3}$ while it is strongly rectifying at $N_{D}=10^{14}-10^{16} / \mathrm{cm}^{3}$. Unlike c-Si, the bandgap of PEDOT:PSS is more likely to contain a broad distribution of defect states arising from the energetic disorder of the amorphous polymer structure [136] that may allow ohmic conduction.

PEDOT:PSS is commonly assumed to be a metallic polymer, considering its high conductivity [36] and high IR reflectivity [35, 147]. On the other hand, it is also known to have p-type conductivity from thermoelectric measurements [148], and it exhibits conductivity-temperature relationship of semiconductor [36]. The 
observation of $n-p^{+}$junction behavior for $n-S i / P E D O T: P S S$ as seen from the RRT and DC-IV measurements is interesting, and suggests that either the semiconducting property is dominant or that the metallic property is suppressed in the hybrid junction. Erickson et al. explained that the absence of thermionic emission could be attributed to the un-pinning of Fermi level at the hybrid interface, which allowed high PEDOT:PSS work function to induce sufficiently high Schottky barrier beyond inversion limit, forming a p-Si layer below the Si surface [39]. The charge separation will happen in the inversion $\mathrm{p}-\mathrm{Si} / \mathrm{bulk} \mathrm{n}$-Si so that the nature of PEDOT:PSS conductivity is not relevant. Jäckle et al. [28] corroborated the presence of an inversion layer, but argued that even considering the Schottky barrier height at the inversion level, the predicted $J_{0}$ is still about 2 - 4 orders of magnitude higher and also does not match the variation on $N_{D}$ dependence. This discrepancy means that the charge selectivity of PEDOT:PSS is still important, forming conventional $n-p^{+}$heterojunction [28], with selectivity due to $n$ Si/PEDOT:PSS conduction band offset on top of the internal Si band bending. The width of internal Si band bending may instead play a role in suppressing tunneling current, but its height is not sufficient by itself to suppress thermionic emission.

It should be noted that most of the studies on the junction characteristics of Si/PEDOT:PSS structure used the same starting solution for PEDOT:PSS (Clevios $\mathrm{PH} 1000)$ as what we have used in our work, yet they still report conflicting $\mathrm{p}-\mathrm{n}$ [28, 31] or Schottky junction [29, 30] interpretation. Though there may be some variations in the preparation process, it is believed that by and large the electronic 
properties of the PEDOT:PSS prepared by the different groups are broadly similar. The different reports have characterized the junctions and applied different models to interpret the results accordingly. It is difficult for us to explain why a particular model has been adopted by a given group, except to concur based on the results presented that the model does not contradict the experimental results. Furthermore, there was no critical evaluation in those papers of both models in terms of their validity for the Si/PEDOT:PSS structure. In addition, there was also no detailed study to validate the junction model through investigating Si/PEDOT:PSS junctions with varying parameters, e.g. doping concentration, as what we have done in our study.

In contrast with n-Si/PEDOT:PSS, SOH junction formed between $\mathrm{n}$-Si and metallic polymer polyacetylene does reveal expected ideal Schottky behavior [149] with excellent sensitivity to its work function, indicative of un-pinning of the Fermi level. In the absence of strong carrier selectivity of polyacetylene, the rectification solely depends on the internal Si band bending. On the other hand, the hybrid junction between $\mathrm{n}$-Si and organic p-type semiconductor methyl violet 2B [150] exhibits thermionic emission at lower forward bias voltage and ohmic behavior at higher forward bias [151], which is believed due to the small conduction band offset. Another p-type organic semiconductor material poly(3-hexylthiophene-2,5-diyl) (P3HT) also formed a rectifying junction with n-Si [104]. The n-Si/P3HT interface exhibits trap-assisted recombination and space-charge-limited-current [104] at lower and higher forward bias respectively. The space-charge-limited-current is 
attributed to at the presence of a high density of defect states distributed inside its bandgap. n-Si/P3HT hybrid junction transport is limited by the organic material, and hole diffusion transport does not manifest under higher forward bias, thus forming a non-ideal $\mathrm{p}$ - $\mathrm{n}$ junction. In contrast, diffusion-dominated transport in $\mathrm{n}$ Si/PEDOT:PSS junction results in ideal $n-p^{+}$junction behavior that leverages on the good transport properties of $\mathrm{Si}$.

\subsubsection{Illuminated current density-voltage characteristic}

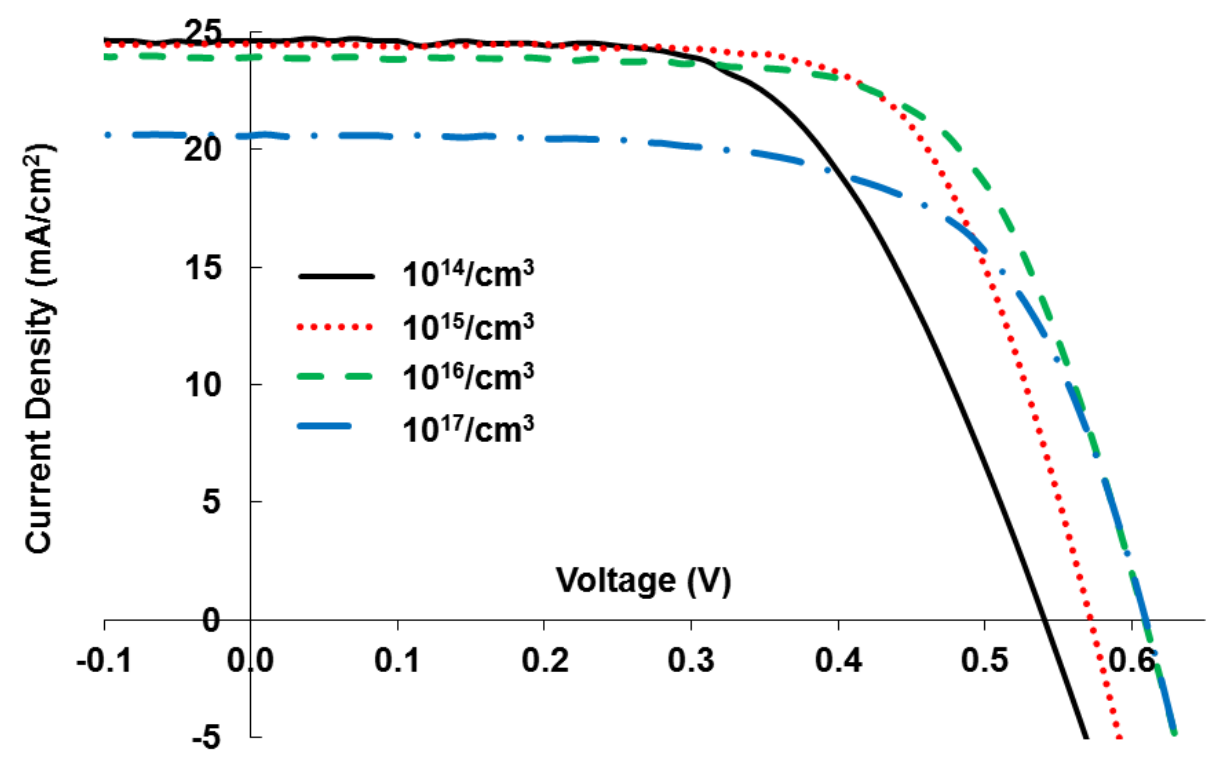

Figure 3.14 : Illuminated J-V response of planar n-Si/PEDOT:PSS junction at different Si substrate doping concentration.

Having established the nature of the hybrid junction, we performed photovoltaic measurement to understand the n-Si/PEDOT:PSS junction performance as a solar cell. Figure 3.14 shows illuminated $\mathrm{J}-\mathrm{V}$ response of samples with different $\mathrm{Si}$ 
substrate doping concentration $N_{D}$ under AM1.5G illumination. The samples are the same as those used for the RRT and dark DC I-V measurements in the previous section.

Table 3.4 : PV Parameters of n-Si/PEDOT:PSS hybrid solar cell at different Si substrate doping concentrations. "Best" sample refer to the sample with the highest PCE for a particular $N_{D}$. The average value is calculated from four samples for each $N_{D}$.

\begin{tabular}{|c|l|l|c|c|c|}
\hline$N_{D}$ & \multirow{2}{*}{ Sample } & \multicolumn{1}{|c|}{$J_{s c}$} & $V_{o c}$ & FF & PCE \\
\cline { 3 - 6 }$\left(/ \mathrm{cm}^{3}\right)$ & & $\left(\mathrm{mA} / \mathrm{cm}^{2}\right)$ & $(\mathrm{V})$ & $(\%)$ & $(\%)$ \\
\hline \multirow{2}{*}{$10^{14}$} & Best & 24.6 & 0.541 & 59.4 & 7.9 \\
\cline { 2 - 6 } & Average & 24.3 & 0.534 & 60.0 & 7.8 \\
\hline \multirow{2}{*}{$10^{15}$} & Best & 24.5 & 0.572 & 68.2 & 9.6 \\
\cline { 2 - 6 } & Average & 24.7 & 0.572 & 65.5 & 9.3 \\
\hline \multirow{2}{*}{$10^{16}$} & Best & 23.9 & 0.609 & 67.2 & 9.8 \\
\cline { 2 - 6 } & Average & 23.5 & 0.605 & 65.4 & 9.3 \\
\hline \multirow{2}{*}{$10^{17}$} & Best & 20.6 & 0.610 & 64.5 & 8.1 \\
\cline { 2 - 6 } & Average & 20.8 & 0.596 & 53.7 & 6.7 \\
\hline
\end{tabular}

The photovoltaic parameters are tabulated in Table 3.4. "Best" sample refer to the sample with the highest PCE for each $N_{D}$. "Average" is the average value calculated from four samples for each $N_{D}$. It can be seen in Table 3.4 that the short circuit current density $\left(J_{s c}\right)$ decreases while the open circuit voltage $\left(V_{o c}\right)$ increases with increasing $N_{D}$. The lower $J_{S c}$ is attributed to the reduction of the diffusion length 
under higher doping which reduces the carrier collection efficiency. $J_{s c}$ monotonously decreases slightly from $10^{14}$ to $10^{16} / \mathrm{cm}^{3}$ due to increased defect recombination, and more significantly from $10^{16} / \mathrm{cm}^{3}$ to $10^{17} / \mathrm{cm}^{3}$ due to increased Auger recombination process at this level of doping. The $V_{o c}$ increase is attributed to the lower saturation current of the cells with higher doping. For abrupt $\mathrm{p}^{+}-\mathrm{n}$ junction, the Shockley ideal diode equation predicts that the saturation current is dominated by minority holes in the moderately doped $\mathrm{n}$-type semiconductor. For a cell with higher $\mathrm{n}$-Si doping, the minority carrier concentration at the depletion edge is reduced, resulting in a lower saturation current. In our observation, the cell with $N_{D}=10^{17} / \mathrm{cm}^{3}$ does not exhibit a strong increase in $V_{o c}$, which is due to the increased shunt leakage attributed to the electron tunneling current [132] from the $\mathrm{Si}$ conduction band to the unoccupied electronic states at the bandgap of PEDOT:PSS, across a thinner depletion width due to the higher $N_{D}$. In summary, the open circuit voltage $\left(V_{o c}\right)$ increases from $0.541 \mathrm{~V}$ to $0.610 \mathrm{~V}$, while the short circuit current $\left(J_{s c}\right)$ density decreases from 24.6 to 20.6 as $N_{D}$ increases from $10^{14}$ to $10^{17} \mathrm{~cm}^{-3}$. The fill factor (FF) increases from $59.4 \%$ to $68.2 \%$ as $N_{D}$ increases from $10^{14}$ to $10^{15} \mathrm{~cm}^{-3}$, which can be attributed to the increased $V_{o c}$ and reduced bulk wafer resistance. The FF decreases from $68.2 \%$ to $64.5 \%$ as $N_{D}$ increases from $10^{15}$ to $10^{17} \mathrm{~cm}^{-3}$, which is due to the increased shunt leakage attributed to the electron tunneling current [132]. A maximum power conversion efficiency (PCE) of $9.8 \%$ is obtained for the cell with $N_{D}=10^{16} \mathrm{~cm}^{-3}$. 


\subsection{Conclusion}

In conclusion, we have fabricated planar n-Si/PEDOT:PSS hybrid heterojunction solar cell with simple low-temperature solution-based processing technique and characterized its junction behavior with reverse recovery transient (RRT) and DC I$\mathrm{V}$ measurement. The hybrid junction cells were fabricated with various $\mathrm{n}$-Si doping concentration $\left(N_{D}\right)$ from $10^{14}-10^{17} \mathrm{~cm}^{-3}$. We found that the solar cells exhibit settling time of 8.3 to $23.5 \mu$ s in its RRT response, which provides strong evidence of their p-n junction characteristic, and contradicts the Schottky junction model that is commonly assumed for such Si/PEDOT:PSS heterojunction. The hybrid cell lifetime is noted to decrease from 126.8 to $39.5 \mu$ s with an increased $n$-Si doping concentration, which suggests that the minority carriers are stored on the n-Si side of the hybrid junction that is consistent with an $n-p^{+}$junction model for the heterojunction. The minority carrier lifetime exhibits a dependency on forward bias current injection level due to the trap-saturation effect of the recombination centers at the Si/PEDOT:PSS interface. The DC-IV measurement results of the junction under dark condition are also consistent with the model of diffusion, and trapassisted recombination controlled dark current. The photovoltaic measurements of the cells under AM1.5G $100 \mathrm{~mW} / \mathrm{cm}^{2}$ illumination reveal that the open circuit voltage $\left(V_{o c}\right)$ increases from $0.541 \mathrm{~V}$ to $0.610 \mathrm{~V}$, while the short circuit current $\left(\mathrm{J}_{\mathrm{sc}}\right)$ density decreases from 24.6 to 20.6 as $N_{D}$ increases from $10^{14}$ to $10^{17} \mathrm{~cm}^{-3}$. A maximum power conversion efficiency (PCE) of $9.8 \%$ is obtained at the hybrid solar cell with $N_{D}=10^{16} \mathrm{~cm}^{-3}$. 


\section{Chapter 4. Simulation and optimization of n-Si/PEDOT:PSS hybrid solar cell with various device architectures}

In this chapter, we investigate n-Si/PEDOT:PSS hybrid solar cells with various device architectures using numerical simulation. We assume n-Si/PEDOT:PSS act as an $n-p^{+}$junction in our simulation, as we previously established in our characterization work in Chapter 3. The simulation is conducted using the numerical device simulation software from Silvaco ATLAS $^{T M}$. Four solar cell device architectures have been studied, which include the conventional front junction (FJ) cell, back junction (BJ) cell, interdigitated back contact (IBC) cell and FJ with an antireflection coating (ARC) compensated layer (FJ-ARC) cell. The photovoltaic performances are simulated as a function of the key device parameters, which include doping of the Si active layer, the layer thickness, and contact geometry. The results are compared and analyzed in relation to the device architecture, and optimizations are performed to achieve the best photovoltaic performance for each type of the solar cell structures.

\subsection{Introduction and motivation}

Si/PEDOT:PSS hybrid solar cell is attractive as a low cost solar cell, owing to its simple solution based and low temperature fabrication process. The present challenge in the research of Si/PEDOT:PSS hybrid solar cell is in improving its efficiency, which is important towards its practical application. Recently, 
Si/PEDOT:PSS hybrid solar cell with a back junction (BJ) structure has reported a power conversion efficiency (PCE) of 17\% [27]. This PCE is higher than those of front junction (FJ) solar cell with PCE of 14\% [28]. The photocurrent in the FJ cell, which has the PEDOT:PSS located at the front side of $\mathrm{Si}$, is limited by parasitic absorption in the PEDOT:PSS film. Light absorption in PEDOT:PSS does not contribute to photocurrent, due to its low exciton diffusion length. By depositing PEDOT:PSS at the back side of Si in the BJ structure, parasitic optical absorption can be significantly reduced [27] since the PEDOT:PSS is placed away from the incident light. Recently, Zhang et al. [41], also reported BJ hybrid cell structure employing a-Si:H passivation scheme with PCE of $16 \%$, signifying that the use of BJ structure to reduce parasitic optical absorption is gaining a prominence.

Despite the promising results obtained for the BJ cell, there are some drawbacks about such device architecture. Due to the presence of front metal electrodes on $\mathrm{Si}$, the front surface passivation is more demanding in the $\mathrm{BJ}$ cell to ensure minimum carriers recombination. For example, the reported BJ cell employed diffusion doped front surface field, and atomic layer deposition (ALD) based $\mathrm{AIO}_{\mathrm{x}}$ tunneling passivation layer at the Si/cathode contact [27]. Such features are in contrary to the goal of realizing solar cell based on low temperature, simple and high throughput process. Besides, since the Si/PEDOT:PSS cell junction is situated away from the incident light, it imposes a stricter demand on the quality of Si to ensure high minority carrier lifetime, such that the carriers generated mainly near 
the front of the cell can be transported to the junction at the back with minimum recombination.

In this chapter, we propose an alternative approach to address the parasitic optical absorption problem in hybrid Si/PEDOT:PSS solar cell without resorting to the use of the more demanding BJ structure but instead based on the simpler FJ architecture. In contrast to the thicker PEDOT:PSS used in typical FJ cell of $\sim 80$ $\mathrm{nm}$, we propose to use a thinner layer of PEDOT:PSS to reduce parasitic absorption, while adding a $\mathrm{Si}_{3} \mathrm{~N}_{4}$ anti-reflection-coating (ARC) layer on top so as to satisfy the refractive index geometric mean rule to minimize optical reflection. However, note that there is a limit to how thin the PEDOT:PSS layer can be, as this will increase the lateral transport resistance of the solar cell. Our device simulation reveals an optimum PEDOT:PSS thickness of $30 \mathrm{~nm}$ and $\mathrm{Si}_{3} \mathrm{~N}_{4}$ thickness of $40 \mathrm{~nm}$ that results in a remarkable PCE of $18.4 \%$. This result is almost similar to the PCE that we have obtained for the best BJ cell simulated in this work of $18.8 \%$. Therefore, the result suggests that our proposed FJ cell can have comparable performance to the BJ cell while eliminating the need for demanding front surface passivation and high Si material quality. Our proposed modified FJ Si/PEDOT:PSS hybrid cell has the potential to deliver high PCE while maintaining its low cost advantage, and is important towards the realization of low cost and high efficiency solar cell. 
In this work, we also investigate the design of Si/PEDOT:PSS hybrid solar cell with interdigitated back contact (IBC) structure. In contrast to the FJ and BJ structures, in IBC structure both the hole and electron collecting contacts are located on the back side of the device. This structure reduces parasitic absorption as well as eliminates grid shadowing as there are no front contacts involved. Besides, since the front surface is free of contacts, a high quality passivation and ARC layer can be developed for the front surface, in a one step process over a large area. In our study, the effect of back contact geometry is simulated to optimize minority and majority carrier collection. The optimum thickness of the $\mathrm{Si}$ absorber layer is also determined by optimizing between photon absorption and carrier collection. Our simulation results indicate that a very high PCE $=20.4 \%$ can be achieved for the Si/PEDOT:PSS hybrid solar cell with IBC structure with an optimized Si absorbing layer thickness of $40 \mu \mathrm{m}$.

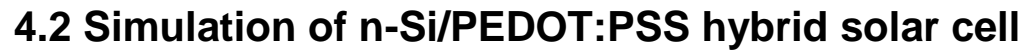 4.2.1 Optical-electrical simulation}

The optical and electrical simulation of the n-Si/PEDOT:PSS hybrid solar cell was performed using the device simulation software from Silvaco ATLAS ${ }^{T M}$. The optical simulation was performed for the planar device using the transfer matrix method (TMM), based on $0.1 \mathrm{~W} / \mathrm{cm}^{2}$ light intensity with $\mathrm{AM} 1.5 \mathrm{G}$ spectrum radiation, under normal incident from the top side of the cell. The solution of the optical simulation was used to calculate the photogeneration rates at different locations in the device, from which the photogenerated current at each bias voltage is determined using 
Poisson, continuity, and generation-recombination equations. The optical constants of PEDOT:PSS are adopted from the literature [27] which were determined using ellipsometry by Zielke et al. The spectroscopty ellipsometry data fitting used a dominant Gaussian oscillator in the UV-region, a Lorentz oscillator in the IR-region and four additional broad Gaussian oscillators to account the absorption in visible and NIR range. The optical constant of Si and $\mathrm{Si}_{3} \mathrm{~N}_{4}$ are taken from optical measurement database [152].

\subsubsection{PEDOT:PSS and $n$-Si electronic properties}

In our simulation, the polymer PEDOT:PSS is modelled as a semiconductor based on the facts that its conductivity increases with temperature [36], while its thermoelectric measurement revealed positive charge dominated conductivity [148], both of which support its nature as a p-type semiconductor material. There exist alternative views that suggest PEDOT:PSS behaves like a metal since it can conduct electricity very well [36] and strongly reflect infrared light [35, 147], and nSi/PEDOT:PSS structures have been modelled as a Schottky junction [29, 30, 153]. However, recently minority carrier dominated transport characteristic has been observed for n-Si/PEDOT:PSS hybrid junction, which suggests that it behaves as

a conventional $p^{+}-n$ junction $[28,31,154]$ and this evidence strongly supports the semiconductor characteristic of PEDOT:PSS. 


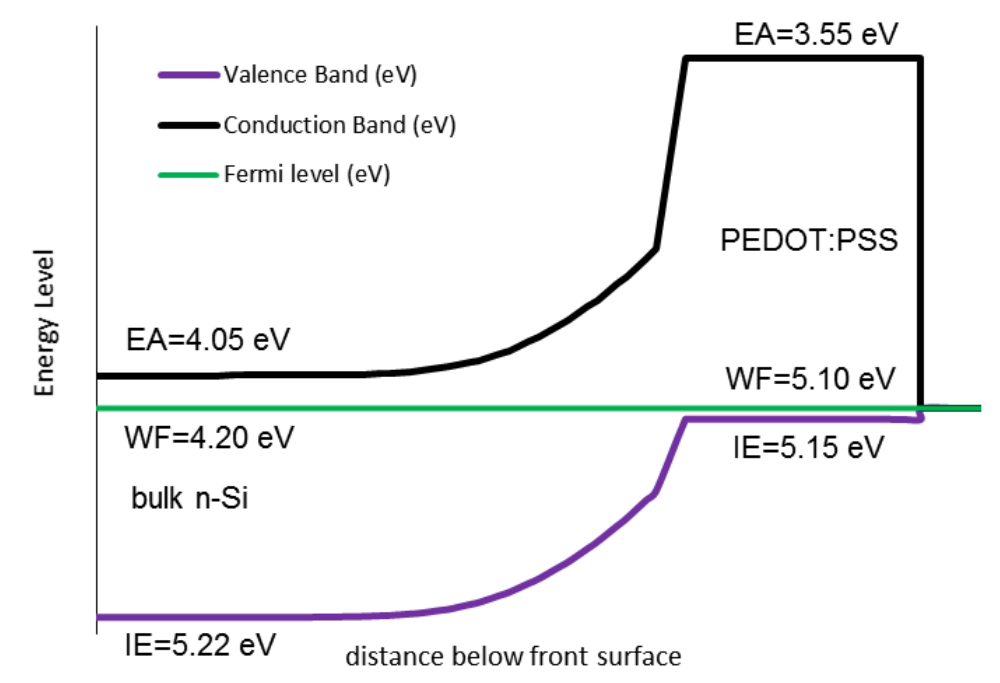

Figure 4.1 : Band diagram of n-type Si/PEDOT:PSS heterojunction, for Si doping concentration $N_{\mathrm{D}}=1 \times 10^{17} / \mathrm{cm}^{3}$. The electron affinity (EA), work function (WF) and ionization energy (IE) of the two materials are indicated.

Figure 4.1 shows the energy band diagram of $n$-Si/PEDOT:PSS structure based on a model of the $p^{+}-n$ heterojunction. In this work we model PEDOT:PSS as a $p$-type semiconductor with a bandgap of $1.6 \mathrm{eV}[155,156]$, electron affinity of $3.55 \mathrm{eV}$ $[154,157]$, effective density of states for conduction and valence bands of $2 \times 10^{21}$ $/ \mathrm{cm}^{3}[136,154,158]$ and acceptor concentration of $3 \times 10^{20} / \mathrm{cm}^{3}[136]$. We assume for PEDOT:PSS a hole mobility $\mu_{\mathrm{p}}$ of $20 \mathrm{~cm}^{2} / \mathrm{Vs}$ that was inferred from conductivity measurement [136] and an electron mobility $\mu_{\mathrm{n}}$ of $1 \mathrm{~cm}^{2} / V \mathrm{~s}$ deduced from solar cell $\mathrm{J}-\mathrm{V}$ characteristics fitting [154]. Based on an electron diffusion length $L_{\mathrm{n}}$ of $10 \mathrm{~nm}$ that is reasonable for conjugated polymer [87, 88], we deduced an electron minority carrier lifetime $\tau_{n}$ of $40 \mathrm{ps}$. The low diffusion length of electrons in PEDOT:PSS accounts for the poor photocurrent generation in PEDOT:PSS and hence the 
parasitic absorption of incident light that does not lead to photocurrent generation. The electronic properties of PEDOT:PSS assumed in the simulation are presented in Table 4.1.

Table 4.1 : The electronic properties of PEDOT:PSS.

\begin{tabular}{|c|c|c|c|}
\hline Parameters & Value & Unit & Reference \\
\hline Bandgap & 1.6 & $\mathrm{eV}$ & {$[155,156]$} \\
\hline Electron affinity & 3.55 & $\mathrm{eV}$ & {$[154,157]$} \\
\hline $\begin{array}{l}\text { Effective density of states for } \\
\text { conduction band }\end{array}$ & $2 \times 10^{21}$ & $/ \mathrm{cm}^{3}$ & {$[136,154,158]$} \\
\hline $\begin{array}{l}\text { Effective density of states for } \\
\text { valence band }\end{array}$ & $2 \times 10^{21}$ & $/ \mathrm{cm}^{3}$ & {$[136,154,158]$} \\
\hline Donor doping concentration & 0 & $/ \mathrm{cm}^{3}$ & \\
\hline Acceptor doping concentration & $3 \times 10^{20}$ & $/ \mathrm{cm}^{3}$ & [136] \\
\hline Hole mobility & 20 & $\mathrm{~cm}^{2} / \mathrm{Vs}$ & [136] \\
\hline Electron mobility & 1 & $\mathrm{~cm}^{2} / \mathrm{Vs}$ & [154] \\
\hline Lifetime & 40 & ps & $\begin{array}{l}\text { Calculated from } \\
\text { diffusion length [87, } \\
88] \text { and electron } \\
\text { mobility[154]. }\end{array}$ \\
\hline
\end{tabular}

We model the bandgap, electron affinity, density of states, and mobility of the bulk Si based on standard Silvaco ATLAS ${ }^{\text {TM }}$ library. The bulk $\mathrm{Si}$ lifetime $\tau_{\text {bulk }}$ is controlled by Shockley-Read-Hall (SRH) recombination lifetime $\tau_{\mathrm{SRH}}$ and Auger 
recombination lifetime $\tau_{\text {Auger }}$ as described in Eq. 4.1. The $\mathrm{SRH}$ lifetime at low doping concentration $\left(\tau_{0, \mathrm{SRH}}\right)$ is set at $1 \mathrm{~ms}$ to model $\mathrm{n}$-type $\mathrm{Si}$ wafer with high electronic quality. The influence of doping concentration $N_{D}$ on $\tau_{S R H}$ is modelled with the concentration dependent SRH model [159-161] as described in Eq.4.2 with fitting parameter $N_{\mathrm{SRH}}$ is taken at default $\mathrm{ATLAS}^{\mathrm{TM}}$ value of $5 \times 10^{16} \mathrm{~cm}^{-3}$, and $N$ is total doping of both donor and acceptor. The bulk Si doping concentration is optimized to ensure low dark saturation current and low bulk resistivity. The effects of doping

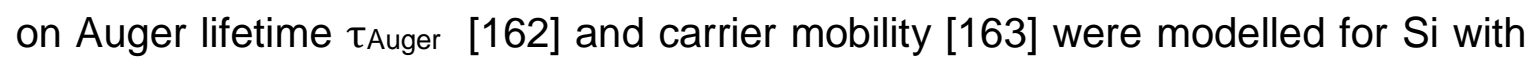
parameters taken from the default Silvaco ATLAS ${ }^{\mathrm{TM}}$ library. Both the front and back surfaces of the Si layer are modelled as a passivated surface with the surface recombination velocity SRV $=10 \mathrm{~cm} / \mathrm{s}[164-166]$.

$$
\begin{gathered}
\frac{1}{\tau_{\text {bulk }}}=\frac{1}{\tau_{S R H}}+\frac{1}{\tau_{\text {Auger }}} \quad \text { Eq. } 4.1 \\
\tau_{S R H}=\frac{\tau_{0, S R H}}{1+N / N_{S R H}} \quad \text { Eq. } 4.2
\end{gathered}
$$

\subsubsection{Devices schematic}

In this section, we present the solar cell architectures for the four types of $n$ Si/PEDOT:PSS hybrid solar cells simulated in our work. They include conventional front junction (FJ) cell, back junction (BJ) cell, interdigitated back contact (IBC) cell and FJ with an antireflection coating (ARC) compensated layer (FJ-ARC) cell. 


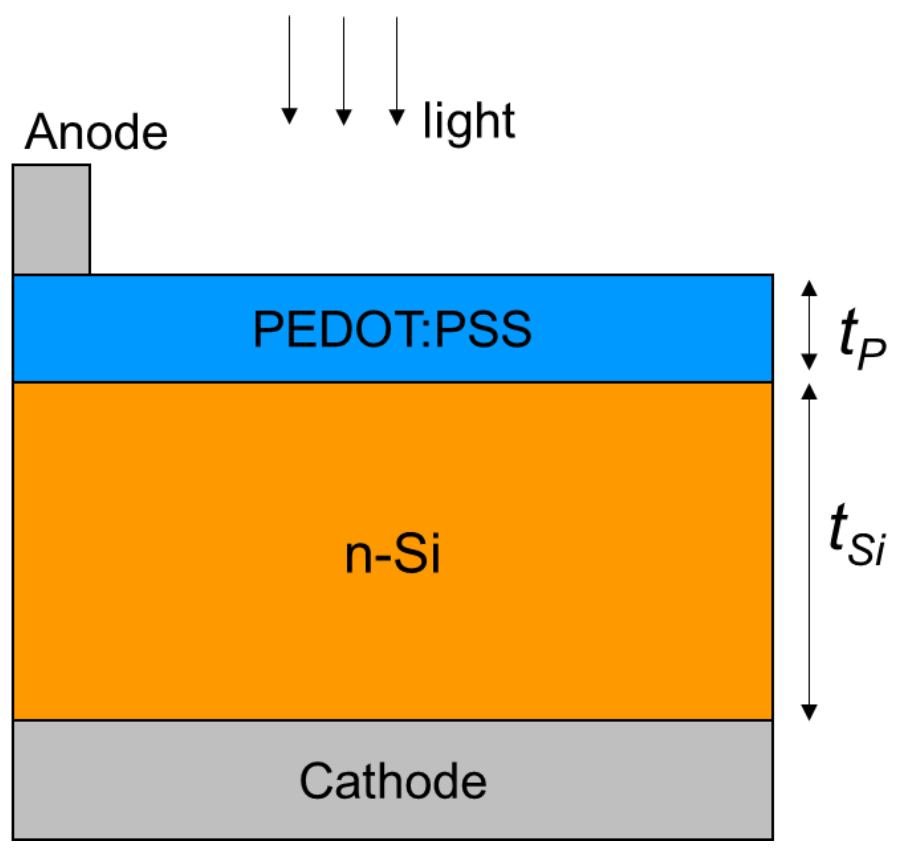

Figure 4.2 : Cross-section of n-Si/PEDOT:PSS hybrid solar cell with conventional front junction (FJ) structure.

Figure 4.2 shows the cross-section of a planar n-Si/PEDOT:PSS hybrid solar cell with conventional FJ structure. The PEDOT:PSS thickness $\left(t_{P}\right)$ is varied to study its anti-reflection coating (ARC) property as well as its resistance as a channel for lateral hole transport. For the FJ structure, it is known that optical absorption and hence the PCE increase monotonically with the silicon thickness $\left(t_{s i}\right)$. In our study, we fixed $t_{S i}$ at $640 \mu \mathrm{m}$, which corresponds to practical wafer thickness and is thick enough to absorb almost all of the incident solar radiation. The Si substrate doping concentration $\left(N_{D}\right)$ is treated as a variable in the simulation and will be varied to optimize the performance of the solar cell. Some part of the solar cell surface is covered with top metal grid and not exposed to light at all. The area of solar cell impacted by the shadow of the grid is equal to the area of the metal grid when the light is incident normal to the surface. The percentage of grid shadowing is the 
percentage of front surface covered with the metal grid. For our 2D simulation case, the grid shadowing is calculated based on the width to periodicity ratio of the finger grid. We set the width of the anode finger ( $\left.W_{\text {anode }}\right)$ and the unit cell period $(P)$ at 60 $\mu \mathrm{m}$ and $1400 \mu \mathrm{m}$, respectively, which will yield $4.3 \%$ grid shadowing.

Figure 4.3 shows the cross-section of a planar n-Si/PEDOT:PSS hybrid solar cell with front junction (FJ) structure having an ARC compensated layer (FJ-ARC) structure. A thin layer of PEDOT:PSS with the thickness $t_{P}$ sits on the Si absorber layer, and a $\mathrm{Si}_{3} \mathrm{~N}_{4} \mathrm{ARC}$ layer with the thickness of $t_{A R C}$ is deposited on top of the PEDOT:PSS to compensate for the lack of the thickness required for minimum reflection. We studied the combination of $t_{P}$ and $t_{A R C}$ that will give the best compromise between achieving good lateral hole transport, minimum parasitic absorption, and strong anti-reflection, so as to maximize the PCE. For the FJ-ARC solar cell, similar to the FJ cell, the Si thickness is set at $640 \mu \mathrm{m}$, while the $\mathrm{Si}$ substrate doping concentration $\left(N_{D}\right)$ is allowed to vary to optimize the performance of the solar cell. For comparison purpose, the design of the top grid structure is also chosen to be the same as that of the FJ cell, with the width of the anode finger $\left(W_{\text {anode }}\right)$ set at $60 \mu \mathrm{m}$ and the width of unit cell period $(P)$ set at $1400 \mu \mathrm{m}$, resulting in the same grid shadowing of $4.3 \%$. A thin layer of PEDOT:PSS layer can be easily achieved by varying the spin coating speed and through solvent post-treatment [167]. $\mathrm{Si}_{3} \mathrm{~N}_{4}$ based $\mathrm{ARC}$ layer can be deposited uniformly on the entire surface, as previously demonstrated by [168]. Ag paste will be deposited on top of ARC layer by screen printing process. Contact between $\mathrm{Ag}$ and PEDOT:PSS can be formed 
by firing the Ag paste to dissolve the intermediate ARC layer and drive the $\mathrm{Ag}$ to contact PEDOT:PSS layer. A similar formation of front contact Ag grid through ARC layer has been commonly applied in commercial screen printing solar cell fabrication [169] without any photolithography process.

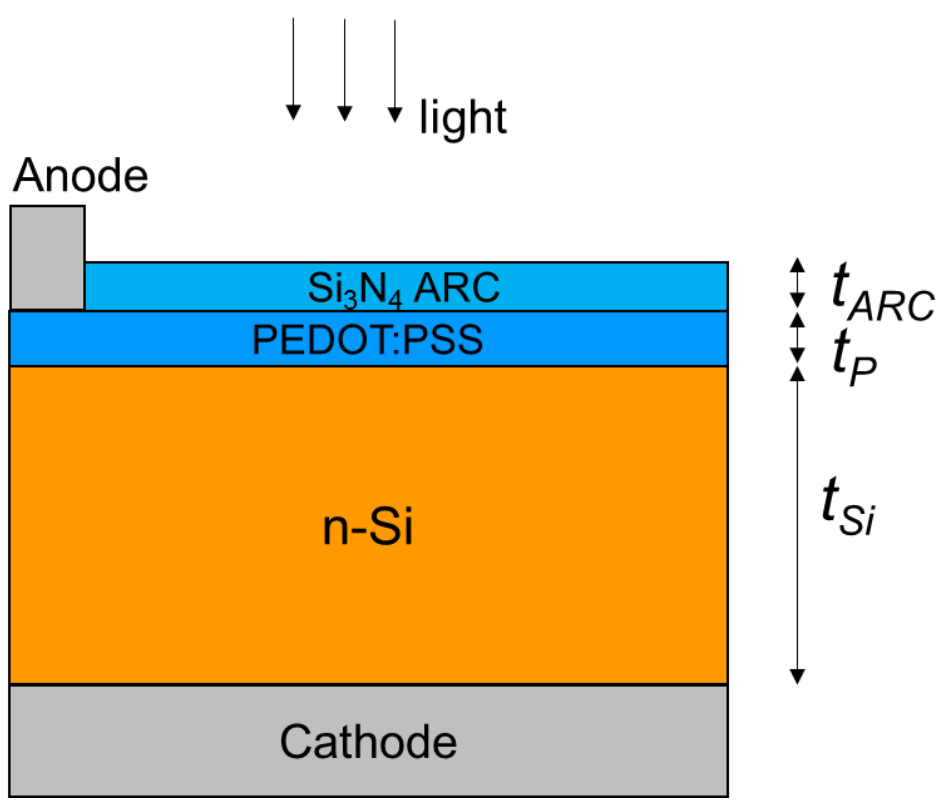

Figure 4.3 : Cross-section of n-Si/PEDOT:PSS hybrid solar cell with front junction and ARC compensated layer (FJ-ARC).

Figure 4.4 shows the cross-section of planar n-Si/PEDOT:PSS hybrid solar cell with back junction (BJ) structure. The simulation of the BJ structure is important as such a structure at present offers the highest efficiency reported for experimental cells, and our simulation studies will allow us to benchmark our proposed FJ-ARC solar cell with the simulated best BJ solar cell. For the BJ structure, the Si thickness $\left(t_{S i}\right)$ and silicon substrate doping concentration $\left(N_{D}\right)$ will be optimized to ensure optimum short circuit current density and open circuit voltage. Note that unlike the case of 
FJ and FJ-ARC cells, the Si thickness in BJ cell cannot be too large as otherwise, the carriers generated nearer the front surface may not be efficiently collected at the back Si/PEDOT:PSS junction. Hence there is a need to investigate and determine the optimum Si layer thickness. We also aimed to study the variation of $N_{D}$ on the photovoltaic performance of the cell, especially the open circuit voltage $\left(V_{o c}\right)$. Increasing $N_{D}$ will lower the dark saturation current $\left(J_{0}\right)$ which in turn will tend to increase the $V_{o c}$. However, increasing $N_{D}$ will also shorten the carrier diffusion length, which will decrease short circuit current density $\left(J_{s c}\right)$. Therefore, an optimum value of $N_{D}$ will exist in which the power conversion efficiency (PCE) is optimized. We also vary the PEDOT:PSS thickness $\left(t_{P}\right)$ so as to optimize the back surface reflectance. Similar to both FJ and FJ-ARC solar cells, the width of the cathode finger $\left(W_{\text {cathode }}\right)$ and the unit cell period $(P)$ are fixed at $60 \mu \mathrm{m}$ and $1400 \mu \mathrm{m}$, respectively. As the PEDOT:PSS layer is located at the back of Si to minimize parasitic absorption, the BJ cell will require an anti-reflection coating on the front side of the Si layer. We have chosen the commonly used and established $\mathrm{Si}_{3} \mathrm{~N}_{4}$ anti-reflection layer for this purpose, with a thickness $\left(t_{A R C}\right)$ set at $75 \mathrm{~nm}$ to minimize light absorption around the peak solar spectrum of $600 \mathrm{~nm}$ [68]. Double polished n-Si wafer will be needed for actual fabrication of the device to ensure a high quality back and front surface. 


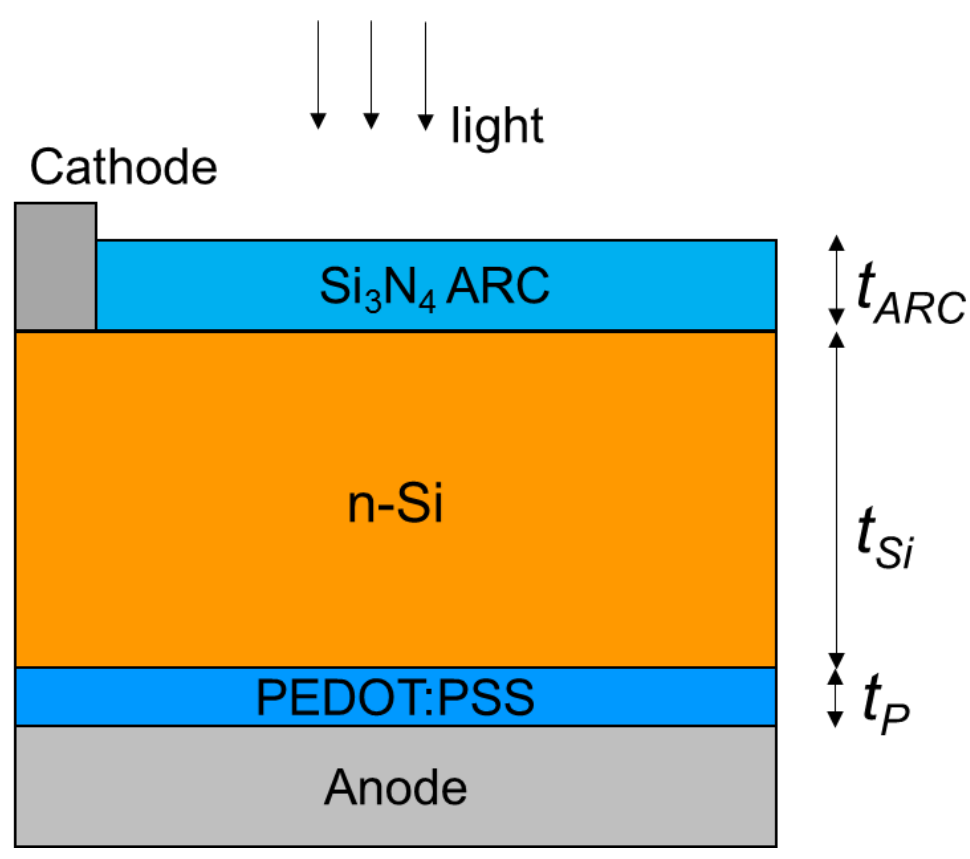

Figure 4.4 : Cross-section of n-Si/PEDOT:PSS hybrid solar cell with back junction (BJ) structure.

Figure 4.5 shows the cross-section of planar n-Si/PEDOT:PSS hybrid solar cell with interdigitated back contact (IBC) structure. The IBC cell is similar to the BJ cell in that the PEDOT:PSS layer is located at the back of Si to minimize parasitic absorption. However, the anode and cathode electrodes are all located at the back of the solar cell to eliminate grid shadowing and concurrently achieve a high quality front surface that is free of metal contacts. For the IBC cell, the silicon thickness $\left(t_{S i}\right)$ and silicon substrate doping concentration $\left(N_{D}\right)$ are set at values that will be derived from the optimization of the BJ solar cell, since they have similar structure and absorption characteristic. The optimized $t_{S i}$ and $N_{D}$ will be determined as a compromise between ensuring sufficient photogeneration, efficient carrier collection and low dark saturation current in the solar cell. A key design of the IBC cell is the layout and spacing of the cathode and anode at the back of the cell. In 
our study, we allow the cathode width ( $\left.W_{\text {cathode }}\right)$, anode width $\left(W_{\text {anode }}\right)$ and the interfinger gap ( $\left.W_{\text {gap }}\right)$ to vary to optimize the minority and majority carrier collection. The minimum widths for $W_{\text {cathode }}, W_{\text {anode, }}$ and $W_{\text {gap }}$ are all set at $60 \mu \mathrm{m}$ to account for the use of practical screen printing technology for metal deposition [170]. Similar to the $\mathrm{BJ}$ cell, a Si $3 \mathrm{~N}_{4}$ anti-reflection coating layer with the thickness $\left(t_{A R C}\right)$ of $75 \mathrm{~nm}$ is introduced on the front surface of the Si layer to minimize light reflection at around the peak solar spectrum. Double polished $\mathrm{n}-\mathrm{Si}$ wafer will be needed for actual fabrication of the device to ensure a high quality back and front surface.

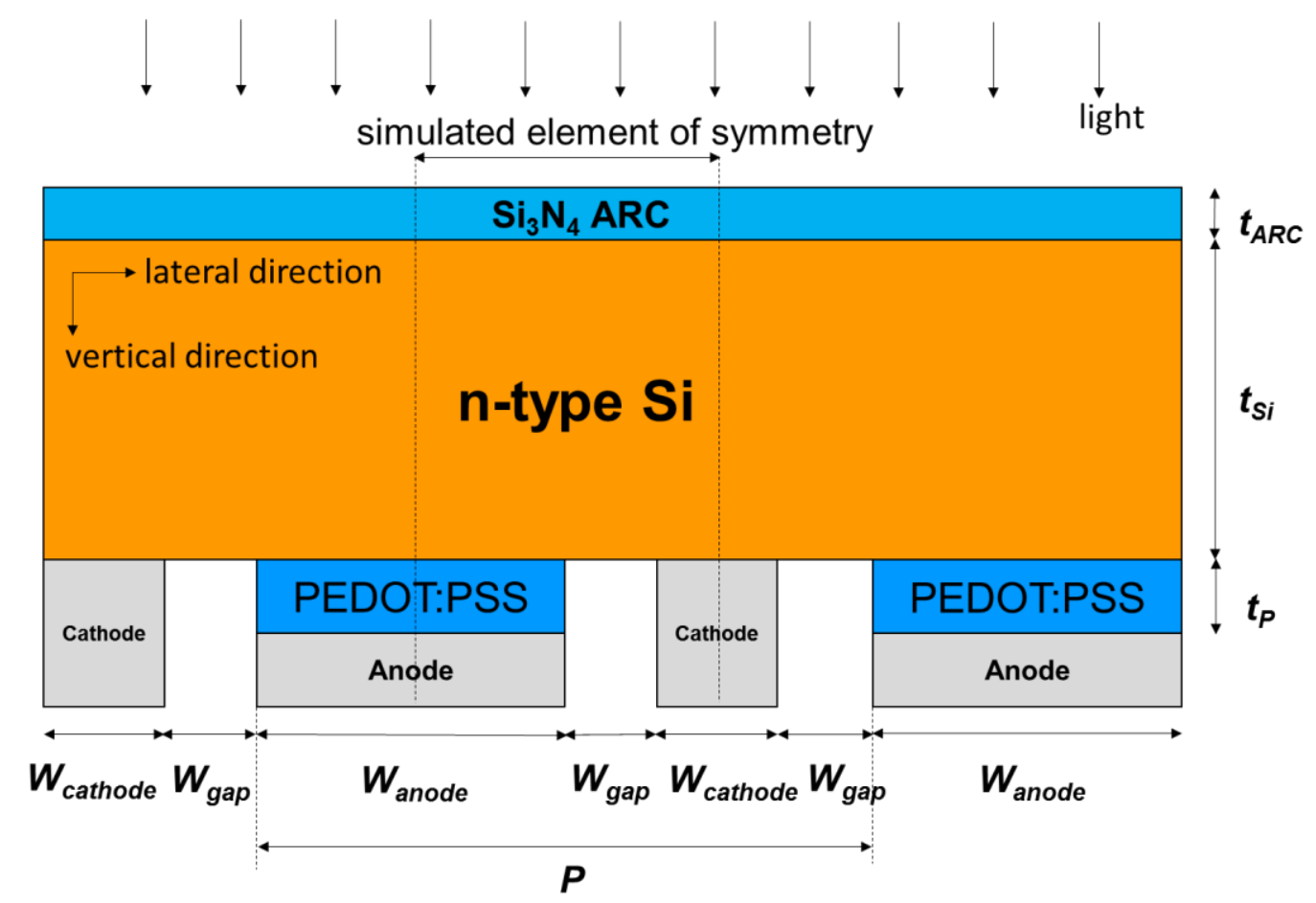

Figure 4.5 : Cross-section of n-Si/PEDOT:PSS hybrid solar cell with interdigitated back contact (IBC) structure. 


\subsection{Conventional and ARC compensated front junction hybrid solar cell}

\subsubsection{Conventional front junction hybrid solar cell}

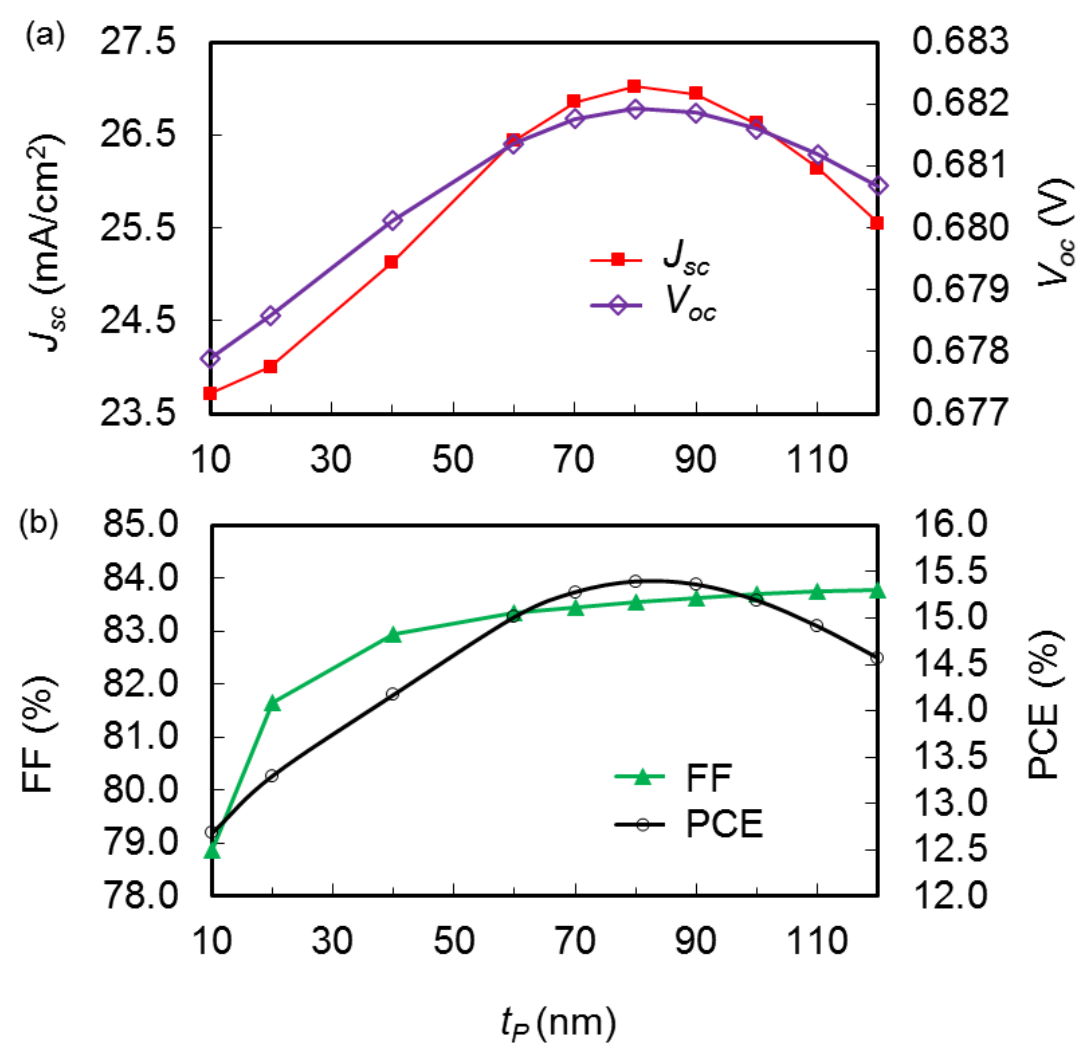

Figure 4.6 : Influence of PEDOT:PSS thickness $t_{P}$ on (a) $J_{s c}$ and $V_{o c}$ and (b) FF and PCE of FJ solar cell with $N_{D}=10^{17} \mathrm{~cm}^{-3}$.

Figure 4.6 shows the influence of PEDOT:PSS thickness $\left(t_{P}\right)$ on the performance of conventional FJ solar cell with $N_{D}=10^{17} \mathrm{~cm}^{-3}$. We found that the short circuit current density $J_{\mathrm{sc}}$ increases with $t_{P}$ from $23.7 \mathrm{~mA} / \mathrm{cm}^{2}$ to $27.0 \mathrm{~mA} / \mathrm{cm}^{2}$ over the range of $10 \mathrm{~nm} \leq t_{\mathrm{p}} \leq 80 \mathrm{~nm}$, and decreases at larger $t_{\mathrm{p}}>80 \mathrm{~nm}$. The optimum thickness of $80 \mathrm{~nm}$ for $J_{\mathrm{sc}}$ is lower than what is expected from the quarter wavelength rule for peak solar spectrum of $105 \mathrm{~nm}$, due to parasitic optical 
absorption losses in a thicker layer of PEDOT:PSS. It is also seen that $V_{o c}$ varies slightly from 0.678 to $0.682 \mathrm{~V}$ over the range of $t_{P}$ investigated, attributed to the change in $J_{\text {sc. }}$.

From Fig. $4.6(\mathrm{~b})$ it is seen that the FF increases from $78.8 \%$ to $82.9 \%$ as $t \mathrm{P}$ varies from $10 \mathrm{~nm}$ to $40 \mathrm{~nm}$, and it saturates at $83.8 \%$ after that for the thicker PEDOT:PSS layer. The increase in FF with $t_{\mathrm{P}}$ is due to a reduction in the PEDOT:PSS sheet resistivity with increased thickness. The saturation behavior seen in the FF at larger tp indicates that there is good hole transport in the lateral direction for PEDOT:PSS thicker than $40 \mathrm{~nm}$. The combined behaviors of the above photovoltaic parameters result in a maximum PCE of $15.4 \%$ achieved at $t \mathrm{P}=80$ $\mathrm{nm}$, primarily controlled by the good ARC effect, good lateral electrical transport in PEDOT:PSS, as well as low parasitic optical absorption.

Next, we set tp to be at its optimum value of $80 \mathrm{~nm}$, and investigate the effects of the Si doping concentration $N_{D}$ on the performance of the FJ cell for a given $t_{S i}=$ $640 \mu \mathrm{m}$. The simulated photovoltaic parameters of the cell as a function of $N_{D}$ are shown in Fig. 4.7. The $J_{s c}$ is seen to drop from 28.3 to $25.7 \mathrm{~mA} / \mathrm{cm}^{2}$ with increased $N_{D}$, due to the reduced diffusion length in higher doped Si layer. In contrast, $V_{o c}$ increases from 0.532 to $0.682 \mathrm{~V}$ with $N_{D}$ over the range of $1 \times 10^{14}<N_{D}<1 \times 10^{17} \mathrm{~cm}^{-}$ 3, attributed to reduced dark saturation current. For $N_{D}>1 \times 10^{17} \mathrm{~cm}^{-3}$ the $V_{o c}$ decreases slightly, brought about by the drop in $J_{s c}$. On the other hand, the FF increases monotonically from 71.4 to $83.6 \%$ with $N_{D}$ due to the larger $V_{o c}$ as well 
as lower Si series resistance at higher $N_{D}$. These mechanisms allow a maximum PCE of $15.4 \%$ to be achieved at $N_{D}=1 \times 10^{17} \mathrm{~cm}^{-3}$, as seen in Fig. $4.7(\mathrm{~b})$.

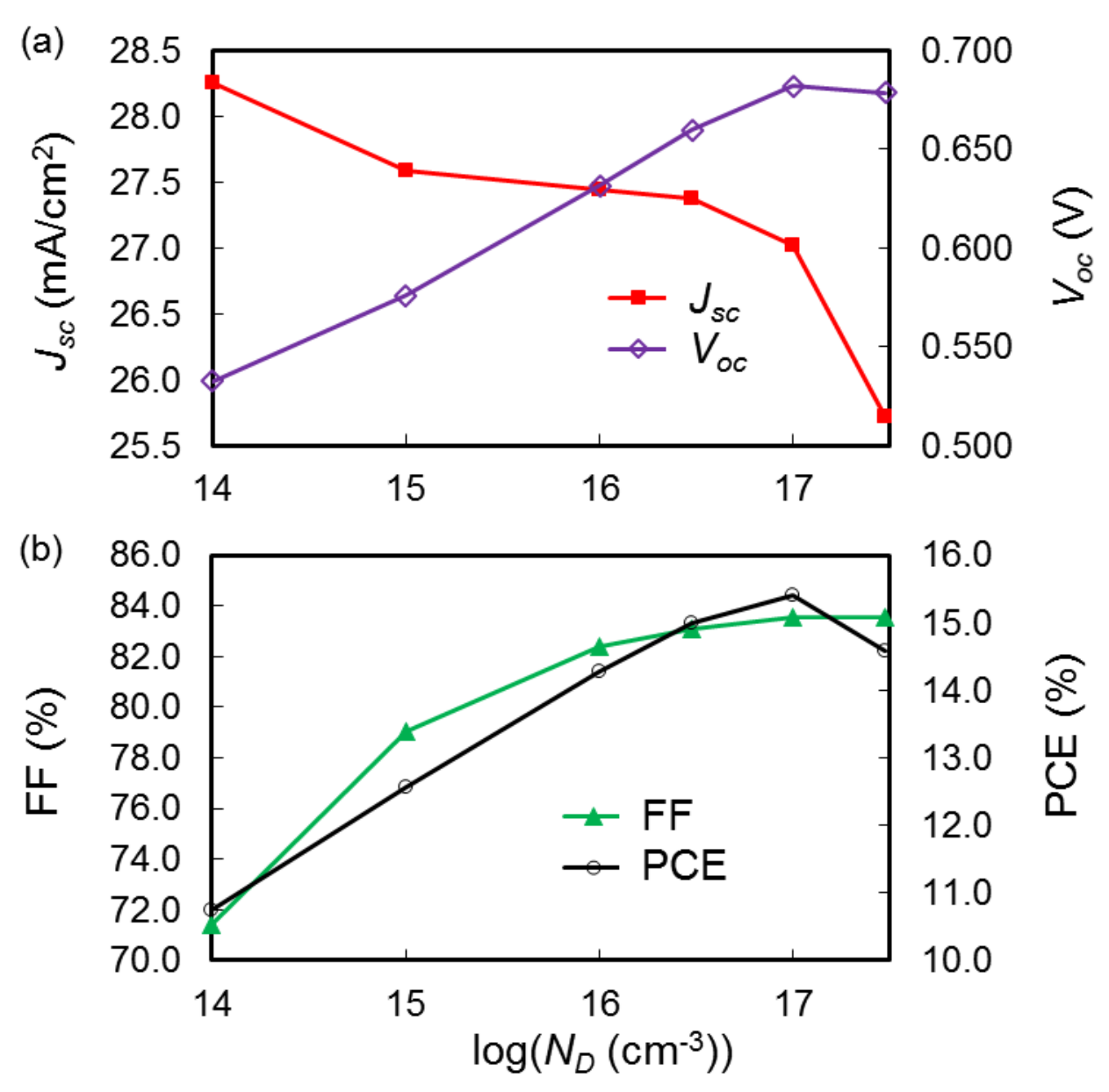

Figure 4.7 : Influence of $\mathrm{Si}$ doping concentration $\left(N_{D}\right)$ on FJ solar cell performance.

We compare the photovoltaic performance between simulation and experimental device as a function of Si doping concentration $\left(N_{D}\right)$ as shown in Fig. 4.8. The I-V curve for the experimental devices for this work had been previously shown in section 3.3.3. It can be seen that the $J_{s c}$ for experimental and simulation devices 
decrease monotonously with $N_{D}$. The $J_{s c}$ from our experimental work is lower than predicted by simulation which is partially attributed to relatively dense front grid spacing on our experimental device, that causes $12 \%$ grid shadowing in comparison to $4.3 \%$ grid shadowing in the simulated devices. It can be seen that for experimental devices $J_{s c}$ drops significantly for $N_{D}>1 \times 10^{16} \mathrm{~cm}^{-3}$, in contrast to the simulation results where $J_{s c}$ only slightly reduced. The significant drop in $J_{s c}$ for the experimental devices can be attributed to low bulk minority carrier lifetime $\tau_{0, \mathrm{SRH}}$ in the experimental device.
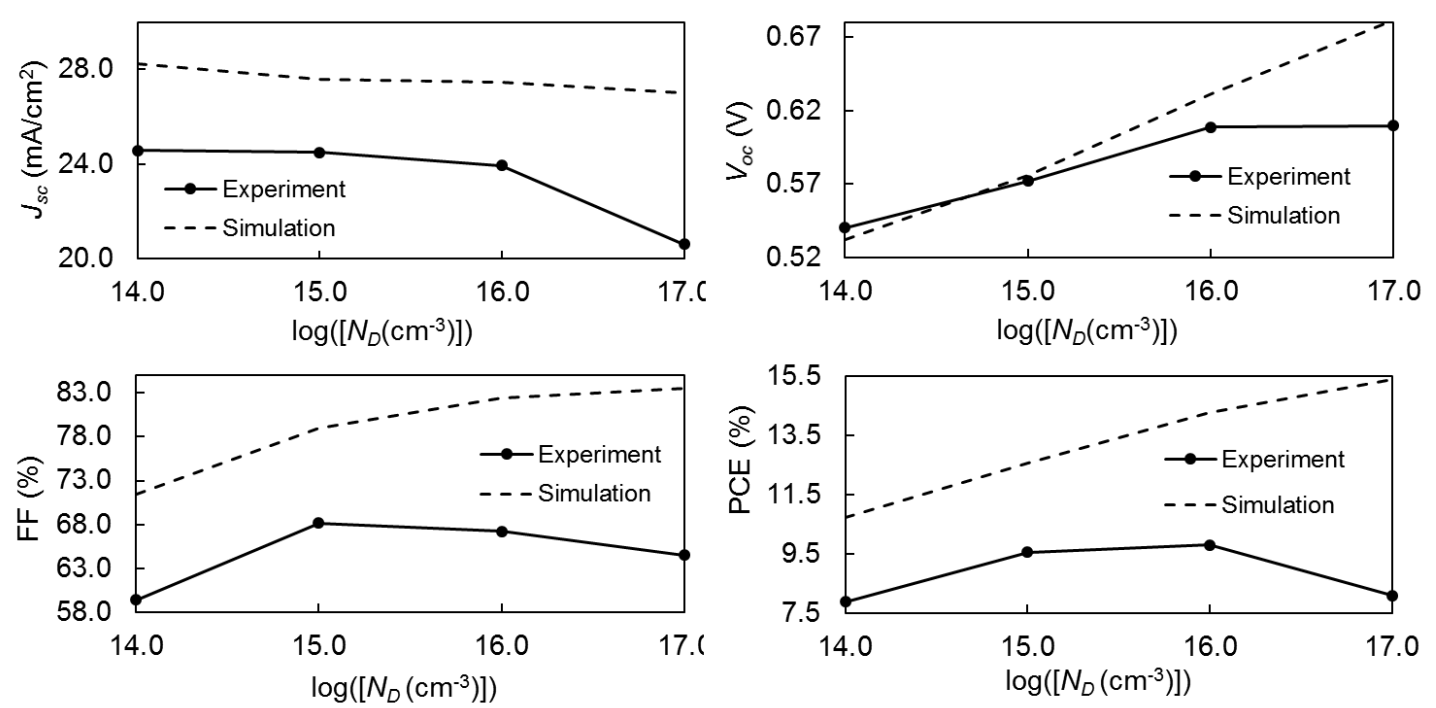

Figure 4.8: Comparison of photovoltaic performance between simulation and experimental device as a function of Si doping concentration $\left(N_{D}\right)$.

We found that the simulation and experimental results agree in the value and trend of $V_{o c}$ as a function of $N_{D}$ for the range of $10^{14}<N_{D}<10^{16} \mathrm{~cm}^{-3}$ as seen in Fig 4.8. However, the experimental $V_{o c}$ does not increase for $N_{D}>10^{16} \mathrm{~cm}^{-3}$, attributed to 
increased shunt leakage. FF in simulation and experimental devices increase with $N_{D}$ at $10^{14}<N_{D}<10^{15} \mathrm{~cm}^{-3}$ range. However, the FF in experimental device decrease at $N_{D}>10^{15} \mathrm{~cm}^{-3}$ in contrast with FF in the simulation that increase with $N_{D}$. We found that the PCE in experiment and simulation increase with $N_{D}$ at $10^{14}$ $<N_{D}<10^{16} \mathrm{~cm}^{-3}$. It is found that at $N_{D}>10^{16} \mathrm{~cm}^{-3}$, the PCE decrease with $N_{D}$ for the experimental devices, in contrast with the simulation devices that increases with $N_{D}$. The decrease in PCE for the experimental device is attributed mainly to the decrease in $J_{s c}$ and FF. Although there are several discrepancies, we considered that the simulation could be reasonably used to design and optimize $n$ Si/PEDOT:PSS hybrid solar cell.

\subsubsection{Front junction hybrid solar cell with ARC compensated layer}

In the previous section, the efficiency of conventional FJ solar cell has been found to be limited by parasitic absorption in the PEDOT:PSS layer. In this section, we investigate the performance of the proposed FJ-ARC cell that uses a thinner layer of PEDOT:PSS to reduce the parasitic absorption while adding a layer of $\mathrm{Si}_{3} \mathrm{~N}_{4}$ ARC layer on top of PEDOT:PSS to ensure minimum reflection of light. $\mathrm{Si}_{3} \mathrm{~N}_{4}$ has been chosen as the ARC material since it is widely used for conventional Si solar cells and exhibits refractive index that satisfies the geometric ratio mean rule. The use of a thinner layer of PEDOT:PSS will increase its lateral resistance and hence an optimum thickness is expected that compromises the need for minimum optical absorption and good lateral hole transport. As the transport behaviors of the FJ and FJ-ARC solar cells and their influences on the photovoltaic performance are similar, 
therefore the optimum $\mathrm{Si}$ doping concentration of $10^{17} / \mathrm{cm}^{3}$ deduced for the conventional FJ cell is also applied here to the FJ-ARC solar cell.
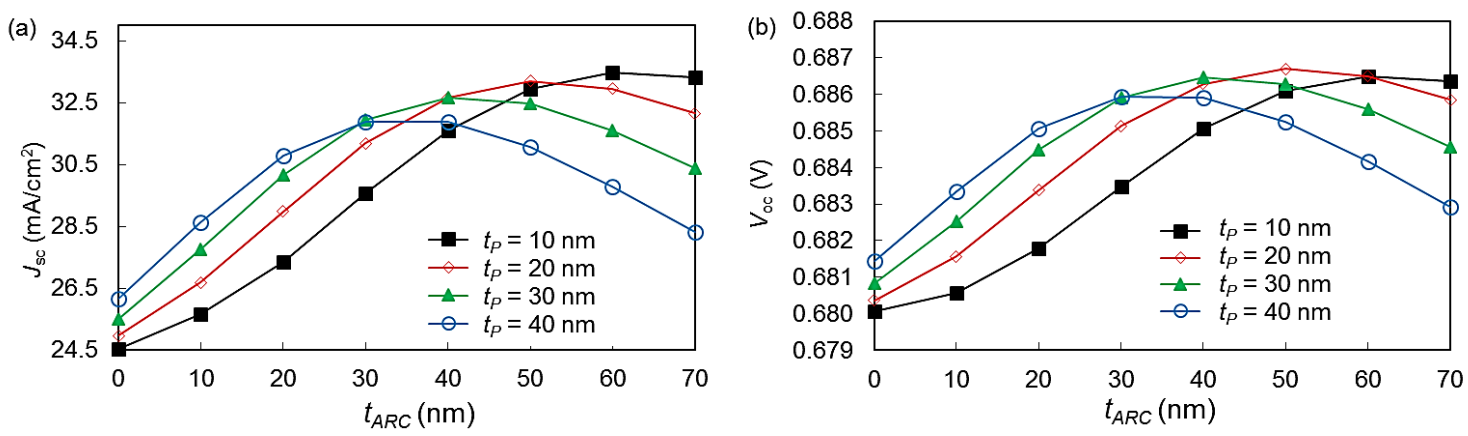

Figure 4.9 : Influence of $t_{P}$ and $t_{A R C}$ on the (a) short circuit current density $\left(J_{s c}\right)$ and (b) open circuit voltage $\left(V_{o c}\right)$ of FJ-ARC hybrid cell with $N_{D}=10^{17} \mathrm{~cm}^{-3}$.

Figure 4.9 shows the combined influence of $t_{P}$ and $t_{A R C}$ on the $J_{S c}$ and $V_{o c}$ of FJARC solar cell. Thin film PEDOT:PSS with $t_{P}=10,20,30$ and $40 \mathrm{~nm}$ are investigated with various thicknesses of $\mathrm{Si}_{3} \mathrm{~N}_{4}\left(t_{A R C}\right)$ deposited on top of it. Figure 4.9(a) shows that there is an optimum $t_{A R C}$ for each value of $t_{P}$ that will maximize $J_{\text {sc. }}$ The optimum $t_{A R C}$ are $60,50,40$, and $30 \mathrm{~nm}$ for tp of $10,20,30$, and $40 \mathrm{~nm}$, respectively. It is noticed that the optimum $t_{A R C}$ is related to $t_{\mathrm{P}}$ by the relation $t_{\mathrm{ARC}} \approx$ $70 \mathrm{~nm}-t \mathrm{p}$. For $t \mathrm{p}=10 \mathrm{~nm}$, the $\mathrm{Jsc}_{\mathrm{sc}}$ is low at $24.5 \mathrm{~mA} / \mathrm{cm}^{2}$ when no $\mathrm{ARC}$ is introduced, and it increases remarkably to $33.5 \mathrm{~mA} / \mathrm{cm}^{2}$ under optimum ARC compensation. The large $J_{\text {sc }}$ obtained for this structure is due to minimum parasitic absorption in the thin $10 \mathrm{~nm}$ PEDOT:PSS layer as well as good coupling of light incident from air into the Si. For larger $t$, the difference in $J_{s c}$ for cells without and with optimum ARC compensation is smaller, since the parasitic absorption effect in the thicker 
PEDOT:PSS is more dominant, hence limiting the improvement in $J_{\text {sc. }}$ Figure $4.9(b)$ shows that the trends in the change of $V_{o c}$ are similar with those of $J_{s c}$, which indicates that the open circuit voltage variation is due to the change in $\mathrm{Jsc}_{\text {sc }}$
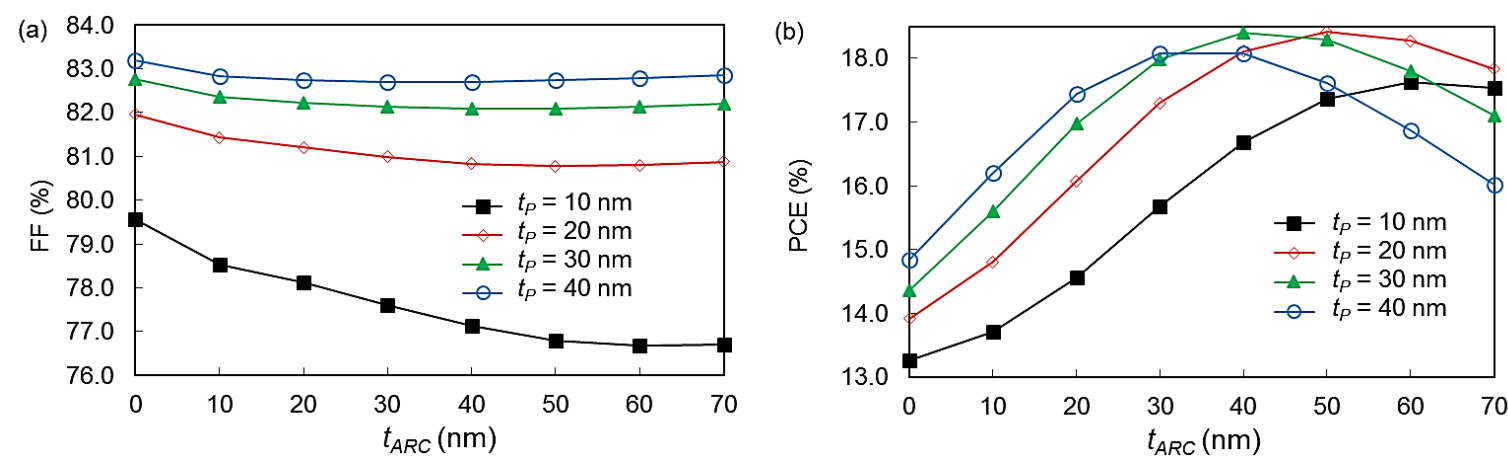

Figure 4.10 : Influence of $t_{P}$ and $t_{A R C}$ on the (a) fill factor (FF) and (b) power conversion efficiency (PCE) of FJ-ARC hybrid cell with $N_{D}=10^{17} \mathrm{~cm}^{-3}$.

Figure 4.10(a) shows the FF of the FJ-ARC solar cell as a function of $t_{A R C}$ and $t$. We found that the smaller the $t_{\mathrm{P}}$, the lower the FF. This is due to the larger sheet resistance of the thinner PEDOT:PSS layer that hinders hole transport in the lateral direction toward the front metallic grid. It is also interesting to note that the FF depends on $t_{A R C}$, particularly for the cell with the thinnest $t_{\mathrm{P}}$ of $10 \mathrm{~nm}$, despite that the $\mathrm{Si}_{3} \mathrm{~N}_{4} \mathrm{ARC}$ layer does not play a direct role in the hole transport. This can be explained by a change in the photocurrent density at the maximum power point $J_{M P}$ which is affected by the presence of the ARC layer and its thickness. For $t_{P}=10$ $\mathrm{nm}$, the influence is the most significant since the sheet resistance is the highest and the variation in the photocurrent is the largest for cells without ARC and with optimum ARC compensation. Figure 4.10(b) shows that the optimum PCE of the 
solar cell is at $18.4 \%$, which can be achieved with tp over the range of 20 to $30 \mathrm{~nm}$ and corresponding $t_{A R C}$ of 40 to $50 \mathrm{~nm}$. This maximum PCE is controlled by a tradeoff between achieving good optical coupling effect, minimum parasitic absorption and minimum sheet resistance. The maximum PCE of $18.4 \%$ obtained for the FJ-ARC is noted to be significantly improved as compared to that of the conventional FJ solar cell of $15.4 \%$. It is also comparable to the PCE of the best back junction (BJ) cell that will be studied in the next section.

\subsection{Back junction and interdigitated back contact hybrid solar cell}

\subsubsection{Back junction hybrid solar cell}
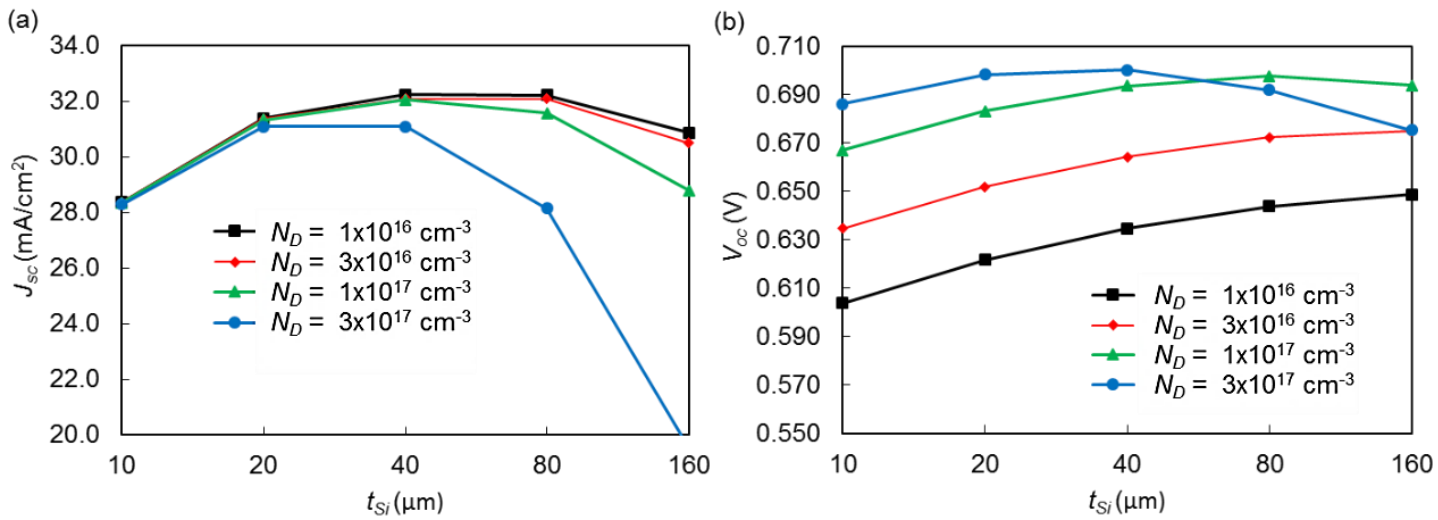

Figure 4.11 : Influence of $t_{S i}$ and $N_{D}$ on the (a) short circuit current density $\left(J_{s c}\right)$ and (b) open circuit voltage ( $\left.V_{o c}\right)$ of BJ solar cell.

Figure 4.11 plots the $J_{s c}$ and $V_{o c}$ of the back junction (BJ) solar cell as a function of the Si thickness $t_{S i}$ under different Si doping concentrations. The back PEDOT:PSS layer thickness $t_{P}$ is set at $70 \mathrm{~nm}$. It is noted that an optimum $t_{S i}$ exists that gives rise to maximum $J_{s c}$ for each doping concentration investigated. The initial increase 
in $J_{s c}$ with $t_{S i}$ is attributed to a stronger optical absorption arising from the use of a thicker Si layer. However, when $t_{s i}$ is further increased, the Si/PEDOT:PSS junction, which is situated at the back side of $\mathrm{Si}$, will be further away from the optically active region at the front surface, resulting in a poorer collection of photogenerated carriers. Hence, at some point, $J_{s c}$ will decrease with increasing $t_{s i}$ despite that there is more optical absorption in the thicker Si. The optimum Si thickness $t_{\text {optimum }}$ that gives rise to maximum $J_{s c}$ is dependent on the minority carrier diffusion length, which in turn is controlled by the doping concentration $N_{D}$. For $N_{D}$ of $(1-3) \times 10^{16}$ $\mathrm{cm}^{-3}, 1 \times 10^{17} \mathrm{~cm}^{-3}$ and $3 \times 10^{17} \mathrm{~cm}^{-3}, t_{\text {optimum }}$ is found to be $\sim 80 \mu \mathrm{m}, 40 \mu \mathrm{m}$, and 20 $\mu \mathrm{m}$, respectively. We observe that a higher $N_{D}$ will lead to a smaller $t_{\text {optimum, }}$ which is expected as the carrier diffusion length is shorter when $\mathrm{Si}$ is heavier doped, and hence the optimum collection of carriers will occur in thinner Si layer.

Figure $4.11(\mathrm{~b})$ shows that over the range of $1 \times 10^{16}<N_{D}<1 \times 10^{17} \mathrm{~cm}^{-3}, V_{o c}$ increases with $N_{D}$. In this range of $N_{D}$ and over the range of $t_{S i}$ investigated, $V_{o c}$ is also noted to increase with $t_{s i}$. This is attributed to the combined effect of increased $J_{s c}$ for $t_{s i}<t_{\text {optimum, }}$ and lower dark saturation current $J_{0}$ as the effect of surface recombination is relatively reduced as compared to bulk Si recombination in thicker Si layer [171]. At larger $t_{S i}=160 \mu \mathrm{m}$, a small reduction is seen in $V_{o c}$ for $N_{D}=1 \times 10^{17}$ $\mathrm{cm}^{-3}$, which is attributed to the drop in $J_{s c}$ seen in Fig. 4.11(a). For the BJ cell with the highest doping of $N_{D}=3 \times 10^{17} \mathrm{~cm}^{-3}$, the $V_{o c}$ increases initially with $t_{S i}$ and subsequently decreases for $t_{S i}>40 \mu \mathrm{m}$. This decrease follows the significant reduction in $J_{s c}$ seen in Fig. 4.11(a) at larger $t_{S i}$. Figure 4.12 shows the FF and PCE 
of the BJ solar cell as a function of the Si thickness $t_{S i}$ under different $S i$ doping concentrations. It is observed in Fig. 4.12(a) that in general, the FF increases with $N_{D}$, which can be attributed to the larger $V_{o c}$ and reduced bulk wafer resistance. Figure 4.12 (b) shows that the BJ cell yield a maximum PCE of $18.8 \%$ for the hybrid cell with Si substrate thickness $t_{S i}$ of $40 \mu \mathrm{m}$ and doping concentration $N_{D}$ of $1 \times 10^{17}$ $\mathrm{cm}^{-3}$.

(a)

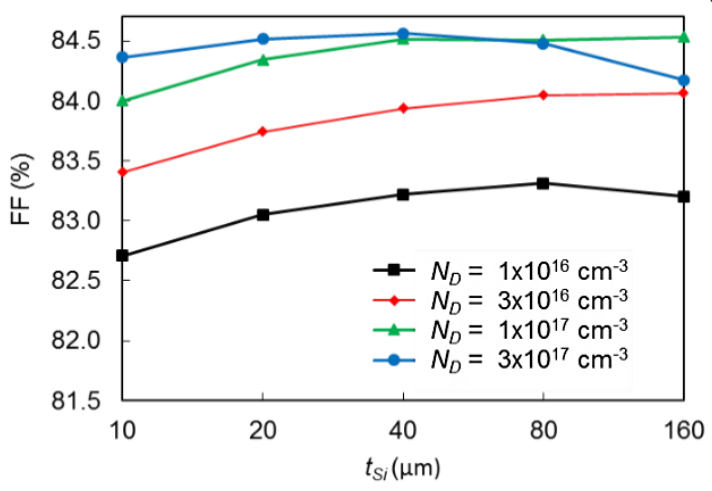

(b)

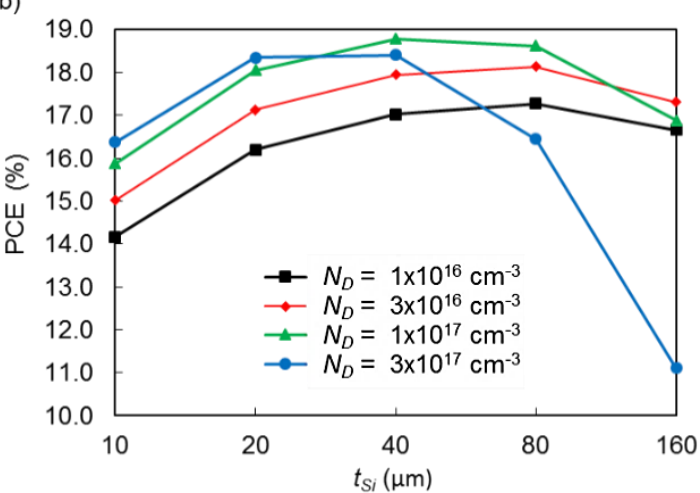

Figure 4.12 : Influence of $t_{S i}$ and $N_{D}$ on the (a) fill factor (FF) and (b) power conversion efficiency (PCE) of the BJ cell.

Based on the optimum Si substrate thickness of $40 \mu \mathrm{m}$ and doping concentration of $10^{17} \mathrm{~cm}^{-3}$ as deduced, we proceed to study the influence of the PEDOT:PSS thickness $t_{P}$ on the BJ cell performance. Figure 4.13 plots the photovoltaic parameters of the BJ cell as a function of $t_{P}$. It is found that $J_{s c}$ increases from 31.6 to $32.0 \mathrm{~mA} / \mathrm{cm}^{2}$ when $t_{P}$ is increased from $10 \mathrm{~nm}$ to $60 \mathrm{~nm}$, which is attributed to improved back surface reflectance. The $J_{s c}$ is relatively independent of $t_{p}$ at $\sim 32.0$ $\mathrm{mA} / \mathrm{cm}^{2}$ in the range of $60<t_{P}<110 \mathrm{~nm}$. The $V_{o c}$ varies slightly according to the 
change seen in $J_{s c}$, while the FF increases slightly due to increased $V_{o c}$ and saturates at large $t_{p}$.
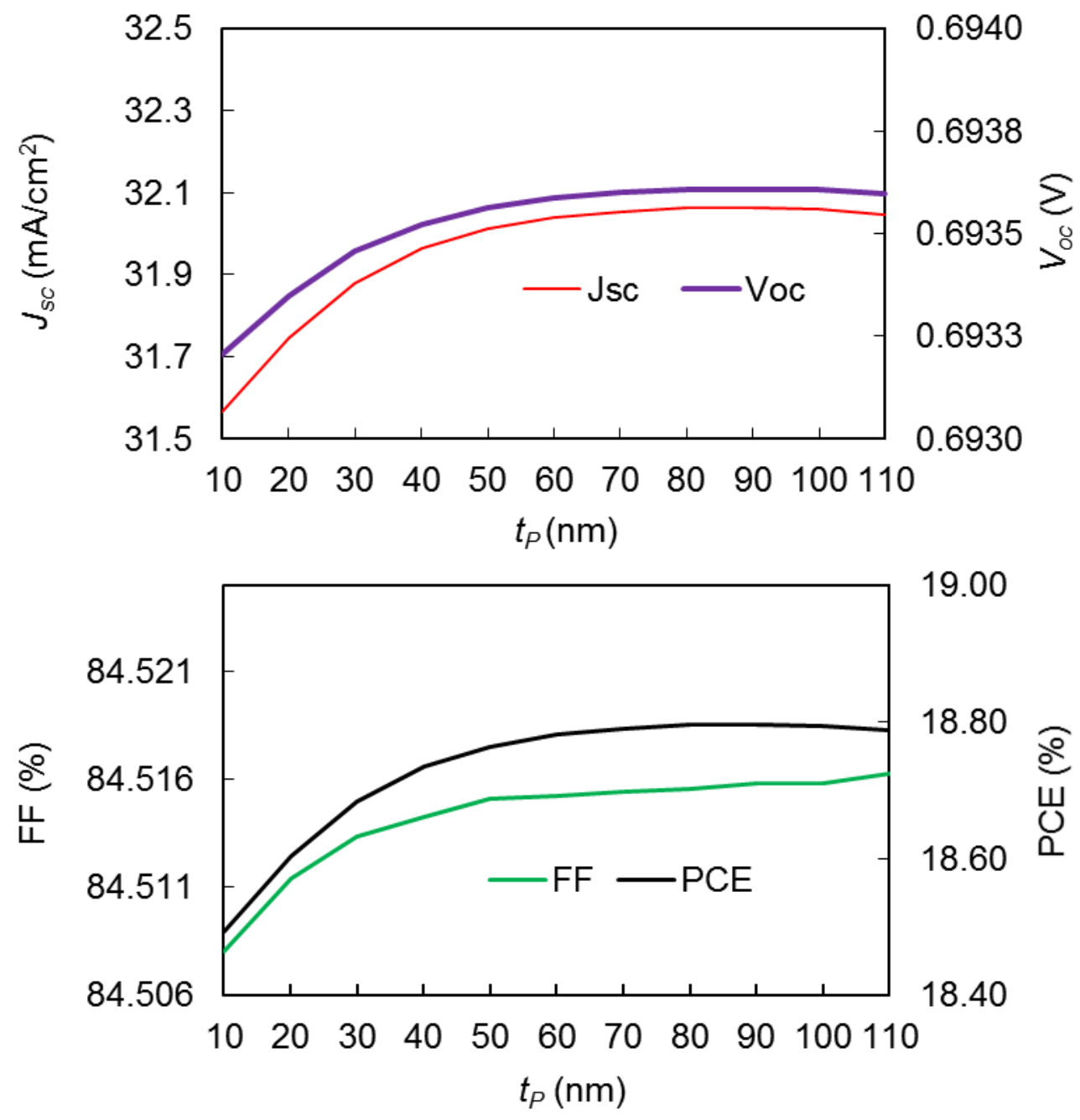

Figure 4.13 : Influence of PEDOT:PSS thickness $\left(t_{P}\right)$ on the BJ cell performance.

Overall, it is seen from Fig. 4.13 (b) that the PCE increases from 18.5 to $18.8 \%$ when $t_{P}$ is increased from $10 \mathrm{~nm}$ to $60 \mathrm{~nm}$, and then saturates at $18.8 \%$ for the optimum PEDOT:PSS thickness range of $60<t_{P}<110 \mathrm{~nm}$. Therefore, we conclude that the PCE of BJ solar cell is not sensitive to the thickness of the PEDOT:PSS 
layer, which is understandable as it is located on the back surface of $\mathrm{Si}$, and thus has a minimum effect on the light absorption.

\subsubsection{Interdigitated back contact hybrid solar cell}

Compared to the back junction solar cell, the interdigitated back contact (IBC) solar cell allows contact free front surface which thus eliminates parasitic absorption and grid shadowing losses. Since it is free of front contact, it allows a high quality passivation layer to be grown on the front surface in one step over a large area. In terms of structure, the IBC cell is similar to BJ cell except that the front cathode grid is moved to the back. Though the majority carriers are now collected at the back, however, the key process in the solar cell, namely, the photogeneration and minority carrier collection would remain the same in both structures. Therefore we set the Si substrate thickness $t_{S i}=40 \mu \mathrm{m}$, and doping concentration $N_{D}=1 \times 10^{17}$ $\mathrm{cm}^{-3}$, which are the optimized values deduced for the BJ cell. Since both the electron and hole collecting contacts are located on the back surface of the IBC cell, an optimum geometry is needed to ensure efficient minority and majority carriers collection. As explained previously, in the simulation of the IBC cell, the

focus is on optimizing the width of the anode $\left(W_{\text {anode }}\right)$ and cathode $\left(W_{\text {cathode }}\right)$ that are responsible for minority hole and majority electron carriers collection, respectively, as well as the width of the gap between the anode and cathode ( $\left.W_{g a p}\right)$, which is needed to prevent shunting between them. 

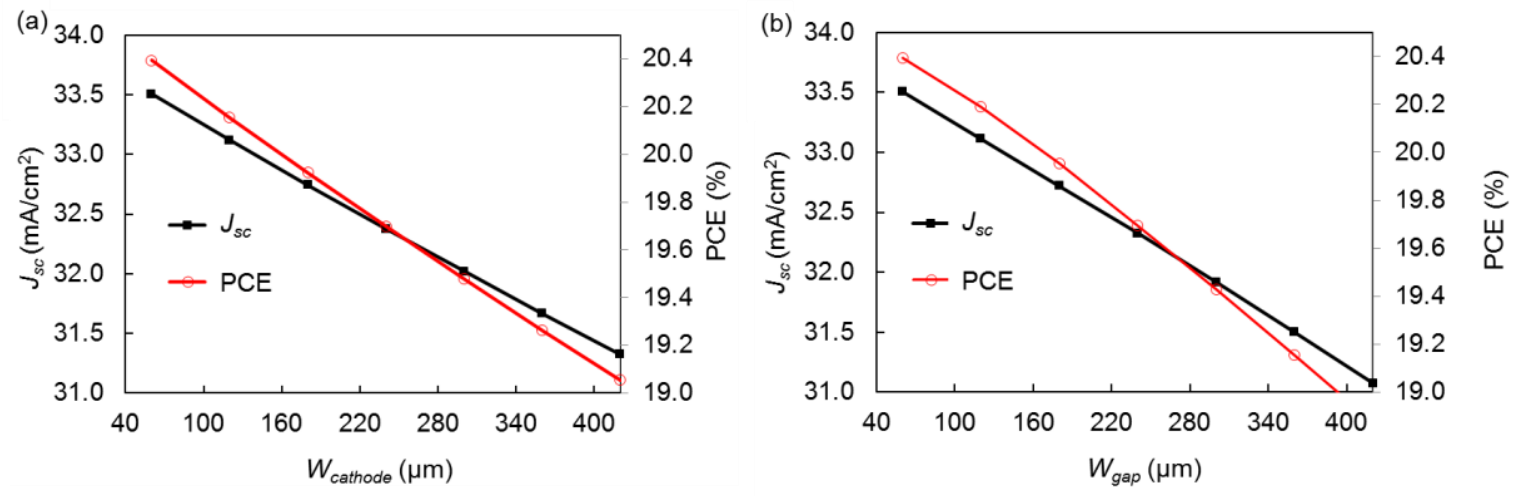

Figure 4.14 : The IBC hybrid solar cell performance (a) as a function of $W_{\text {cathode }}$ for a given $W_{\text {anode }}=5000 \mu \mathrm{m}$ and $W_{\text {gap }}=60 \mu \mathrm{m}$ and (b) as a function of $W_{\text {gap }}$ for a given $W_{\text {anode }}=5000 \mu \mathrm{m}$ and $W_{\text {cathode }}=60 \mu \mathrm{m}$.

It is found that an increase in $W_{\text {cathode }}$ or $W_{\text {gap }}$ leads to a monotonic decrease in $J_{\mathrm{sc}}$ and PCE of the IBC cell. In Fig. 4.14(a) we illustrate the results by plotting the $J_{\mathrm{sc}}$ and PCE for an IBC cell as a function of $W_{\text {cathode, with }} W_{\text {anode }}$ and $W_{\text {gap }}$ fixed at 5000 $\mu \mathrm{m}$ and $60 \mu \mathrm{m}$, respectively. We found that the $J_{\mathrm{sc}}$ and PCE monotonously decrease with $W_{\text {cathode. }}$ In Fig. 4.14(b), we plot the $J_{\text {sc }}$ and PCE for an IBC cell as a function of $W_{\text {gap }}$, with $W_{\text {anode }}$ and $W_{\text {cathode }}$ fixed at $5000 \mu \mathrm{m}$ and $60 \mu \mathrm{m}$ respectively. Similarly, we found that the $J_{s c}$ and PCE monotonously decrease with $W_{\text {gap. }}$. The results can be attributed to the larger carrier collection distance involved when these parameters are increased, which thus decreases the efficiency of carriers collection.

Since we found that the PCE monotonously decrease with $W_{\text {cathode }}$ or $W_{\text {gap }}$, we fixed $W_{\text {cathode }}$ and $W_{\text {gap }}$ at the smallest possible value of $60 \mu \mathrm{m}$ for the subsequent 
simulations, while allowed $W_{\text {anode }}$ to vary, to study its effects on the performance of the IBC cell. The $60 \mu \mathrm{m}$ value for $W_{\text {cathode }}$ or $W_{\text {gap }}$ is determined by the minimum width that can be achieved in practical screen printing technology widely used in commercial solar cell fabrication.
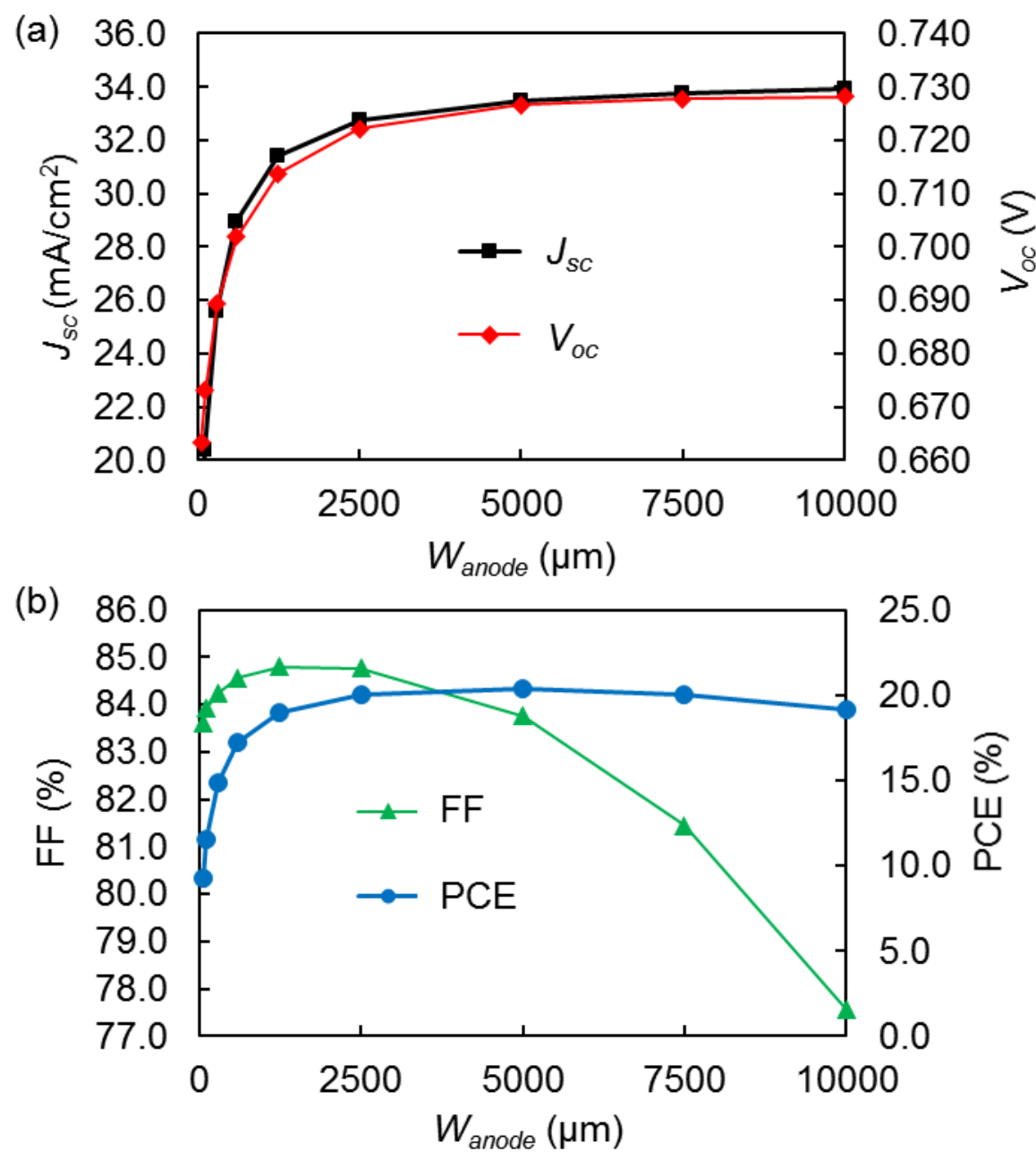

Figure 4.15 : Influence of $W_{\text {anode }}$ on IBC solar cell performance with

$$
W_{\text {cathode }}=W_{\text {gap }}=60 \mu \mathrm{m}
$$


Figure 4.15 displays the photovoltaic parameters of the IBC solar cell as a function of $W_{\text {anode. }}$ We found that $J_{s c}$ increases sharply with $W_{\text {anode }}$ initially and then gradually for $W_{\text {anode }}$ beyond $\sim 2500 \mu \mathrm{m}$. Note that the minority hole carriers that are generated above the anode are more readily collected, while those generated above the cathode will need to diffuse to the neighbouring nearest anodes before they can be collected. When $W_{\text {anode }}$ is increased while $W_{\text {cathode }}$ is maintained constant, the ratio of the area covered by anode to cathode will increase, resulting in more carriers being generated above the anode. Therefore, there will be more carriers that can be readily collected, which contributes to the increase in $J_{\text {sc }}$ as seen in our simulation result. A similar increase in $V_{o c}$ is also observed, which can be attributed to the change in $J_{s c}$ just explained. On the other hand, the FF increases with $W_{\text {anode }}$ and peaks at $85 \%$ at $W_{\text {anode }}=1250 \mu \mathrm{m}$ due to the large $V_{\text {oc. }}$ It then decreases to $78 \%$ at larger $W_{\text {anode, }}$ which is attributed to the larger series resistance as the majority carriers generated above the PEDOT:PSS layer will need to travel a longer distance before being collected at the cathode. Due to the increase in $J_{s c}$ and $V_{o c}$ with $W_{\text {anode, }}$ and the peak behavior exhibited by the FF, the optimum geometry is found to occur at an intermediate $W_{\text {anode }}$ of $5000 \mu \mathrm{m}$, which gives rise to a maximum PCE of $20.4 \%$.

We compare the I-V curves of the best performance cell for each type of cell structures in Fig 4.16. It can be seen that the $J_{s c}$ for the $F J$ cell is the lowest amongst all devices $\left(27 \mathrm{~mA} / \mathrm{cm}^{2}\right)$, which is attributed to parasitic absorption loss. The $\mathrm{Jsc}_{\mathrm{sc}}$ for FJ-ARC and BJ cell is significantly higher than those of FJ cell at $32.7 \mathrm{~mA} / \mathrm{cm}^{2}$ and 
$32.1 \mathrm{~mA} / \mathrm{cm}^{2}$, respectively, due to a reduction in parasitic absorption. Further increase in $J_{\mathrm{sc}}$ at $J_{\mathrm{sc}}$ of $33.5 \mathrm{~mA} / \mathrm{cm}^{2}$ on IBC cell is attributed to the reduction of both parasitic absorption as well as grid shadowing. The $V_{\text {oc }}$ for IBC cell is also the highest at $0.727 \mathrm{~V}$ in comparison to FJ, FJ-ARC and BJ cell which has $V_{o c}$ of 0.682 $\mathrm{V}, 0.686 \mathrm{~V}$, and $0.694 \mathrm{~V}$ respectively. The high $V_{o c}$ and $J_{s c}$ value for IBC cell allow IBC cell to exhibit the highest power output amongst other cell structures.

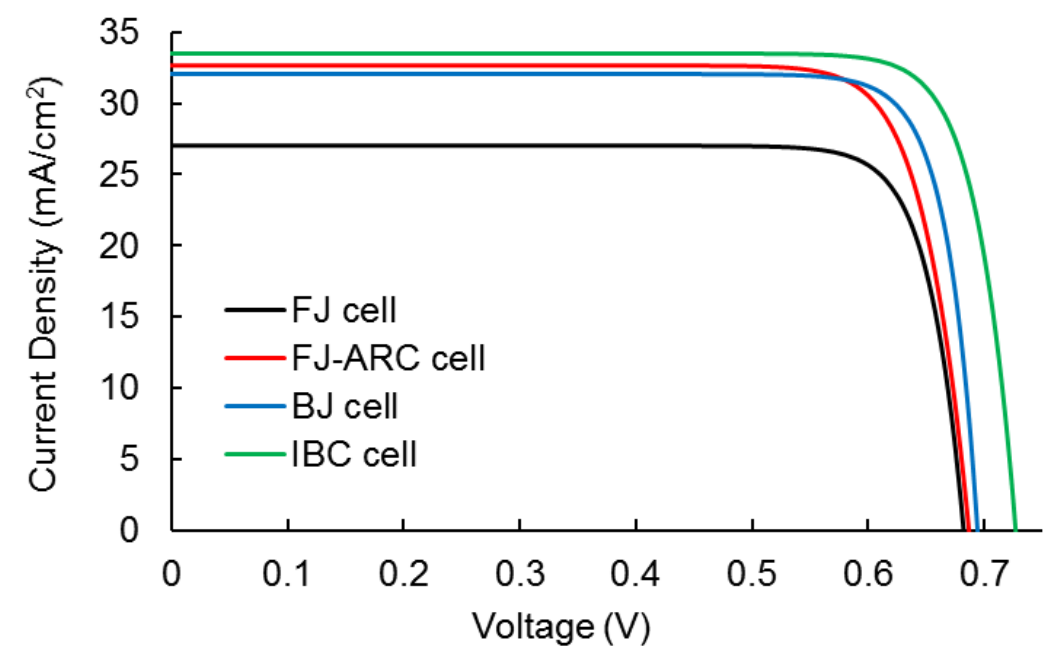

Figure $4.16:$ I-V curves of n-Si/PEDOT:PSS hybrid solar cell with front junction (FJ), front junction with ARC compensated layer (FJ-ARC), back junction (BJ) and interdigitated back contact (IBC) structure.

We summarize the photovoltaic parameters of the best performance cell for each type of cell structures in Table 4.2. We see that for the FJ-ARC solar cell proposed in this work, despite its lower FF due to the higher series resistance in the thinner PEDOT:PSS layer, it exhibits a high PCE of $18.4 \%$, which is significantly improved as compared to that of the conventional FJ cell of $15.4 \%$. Its PCE is also 
comparable to that of the BJ cell of $18.8 \%$, which at the moment is the best cell structure that has been demonstrated experimentally to deliver the highest PCE for Si/PEDOT:PSS hybrid cell [27]. From Table 4.2, we also observe that the IBC cell offers the highest PCE of $20.4 \%$, which is better than both the BJ cell and the FJARC cell. However, it should be noted that the IBC cell has a number of disadvantages such as more complicated patterning process and stricter requirements for minority carrier lifetime compared to FJ-ARC cell.

Table 4.2 : Comparison of hybrid Si/PEDOT:PSS solar cell PV parameters for planar FJ cell, FJ-ARC cell, BJ cell and IBC cell. All cells are based on a planar structure with $\mathrm{N}_{\mathrm{D}}=10^{17} \mathrm{~cm}^{-3}$.

\begin{tabular}{|l|c|c|c|c|}
\hline Hybrid solar cell design & $\begin{array}{c}J_{s c} \\
\left(\mathrm{~mA} / \mathrm{cm}^{2}\right)\end{array}$ & $\begin{array}{c}V_{o c} \\
(\mathrm{~V})\end{array}$ & $\begin{array}{c}\text { FF } \\
(\%)\end{array}$ & $\begin{array}{c}\text { PCE } \\
(\%)\end{array}$ \\
\hline Front Junction cell (FJ) & 27.0 & 0.682 & 83.5 & 15.4 \\
\hline $\begin{array}{l}\text { Front Junction cell with } \\
\text { ARC compensated layer } \\
\text { (FJ-ARC) }\end{array}$ & 32.7 & 0.686 & 82.1 & 18.4 \\
\hline Back Junction cell (BJ) & 32.1 & 0.694 & 84.5 & 18.8 \\
\hline Interdigitated back contact & 33.5 & 0.727 & 83.8 & 20.4 \\
\hline cell (IBC) & & & & \\
\hline
\end{tabular}




\subsection{Conclusion}

In conclusion, we have investigated four solar cell device architectures which include the conventional front junction (FJ) cell, back junction (BJ) cell, interdigitated back contact (IBC) cell and FJ structure with ARC compensated layer (FJ-ARC) cell. We found that planar BJ cell in which the PEDOT:PSS layer is located on the back surface of the Si layer can yield $J_{\mathrm{sc}}$ of $32.1 \mathrm{~mA} / \mathrm{cm}^{2}$ and PCE of $18.8 \%$ under an optimum Si thickness of $40 \mu \mathrm{m}$. These are higher as compared to $J_{\mathrm{sc}}$ of $27.0 \mathrm{~mA} / \mathrm{cm}^{2}$ and PCE of $15.4 \%$ achieved for bulk conventional FJ cell. The higher performance is attributed to the elimination of parasitic absorption in PEDOT:PSS, as well as the introduction of an antireflective coating with a refractive index that better matches the geometric mean ratio between those of $n-S i$ and air. Our simulation has also demonstrated that the parasitic absorption issue affecting the FJ cell architecture can be resolved without resorting to a more demanding $\mathrm{BJ}$ structure. We proposed to adopt the simpler FJ structure but reduce the thickness of the PEDOT:PSS layer to $30 \mathrm{~nm}$ to minimize parasitic absorption and introduce a layer of silicon nitride anti-reflection-coating (ARC) layer with a thickness of $\sim 40$ $\mathrm{nm}$ on top. We obtained a high $J_{\mathrm{sc}}$ of $32.7 \mathrm{~mA} / \mathrm{cm}^{2}$ and PCE of $18.4 \%$ for the proposed FJ-ARC cell structure, which is almost similar to those achieved for the BJ cell. We have also simulated the IBC cell in which both the hole and electron collecting contacts are located on the back side of $\mathrm{Si}$. The device architecture is free of any contact on the front side, which thus eliminates shadowing and parasitic absorption, and allows excellent passivation of the solar cell in one step over a 
large area. The use of the IBC cell structure yields the highest PCE of $20.4 \%$ among the four types of cell structures investigated.

To the best of our knowledge, there is no such comprehensive study and comparison of $n$-Si/PEDOT:PSS hybrid solar cells with the various cell architectures, as what we have carried out in this work. In particular, our proposed FJ-ARC cell which can deliver similar performance as the BJ cell has not been reported and investigated before. As for the IBC cell, though the concept of having all electrodes at the back of $\mathrm{Si}$ has been successfully demonstrated in conventional $\mathrm{Si}$ solar cell, to the best of our knowledge, it has not been applied to $\mathrm{n}$ Si/PEDOT:PSS hybrid solar cell either in simulation study or experimental demonstration. The simulation results obtained in this study are promising and demonstrate the great potential of n-Si/PEDOT:PSS solar cell based on the FJARC and IBC structures. They will also be helpful in guiding the future experimental realization of such n-Si/PEDOT:PSS solar cells. 


\section{Chapter 5. Fabrication and optimization of Si/molybdenum oxide heterojunction solar cell}

In this chapter, we study Si/transition metal oxide (Si/TMO) heterojunction solar cell. Firstly, we describe the motivation for using a transition metal oxide as a selective carrier contact for Si heterojunction solar cell. Subsequently, we investigate experimentally the use of the transition metal oxide, namely molybdenum oxide $\left(\mathrm{MoO}_{\mathrm{x}}\right)$, as the selective carrier contact material for $\mathrm{n}-\mathrm{Si}$ heterojunction solar cells. Planar front junction $\mathrm{n}-\mathrm{Si} / \mathrm{MoO}_{\mathrm{x}}$ heterojunction solar cells are fabricated by e-beam evaporation of $\mathrm{MoO}_{x}$ solid source onto $\mathrm{n}$-Si substrates to form an electron blocking layer. Indium tin oxide (ITO) is then deposited on top of $\mathrm{MoO}_{\mathrm{x}}$ by RF sputtering to serve as an antireflection coating as well as an electrode. We investigate the performance of the $\mathrm{Si} / \mathrm{MoO}_{x}$ solar cell in terms of the $\mathrm{Si}$ substrate doping concentration, and the two types of top electrodes used, namely PEDOT:PSS and ITO. We also study the influence of a water barrier layer on top of $\mathrm{MoO}_{\mathrm{x}}$ which is used to prevent moisture adsorption on $\mathrm{MoO}_{\mathrm{x}}$ in ambient air. Finally, the effect of native $\mathrm{Si}$ oxide at the $\mathrm{n}-\mathrm{Si} / \mathrm{MoO}_{\mathrm{x}}$ interface on the characteristics of the cell is also reported.

\subsection{Introduction and motivation}

Reducing the cost of silicon (Si) solar cell is important in accelerating the growth of the commercial photovoltaic (PV) market. Today, the key challenge in Si solar cell is to achieve high-efficiency cell at a lower cost. To address this issue, two key 
approaches such as low-temperature fabrication process and adoption of thin film $\mathrm{Si}$, are being actively researched [172]. The motivation to achieve low-temperature fabrication has driven the development of Si/organic heterojunction solar cell [26, 102] as shown in Fig. 5.1(a) to replace high-temperature diffusion doping process currently used to form a p-n junction in conventional Si solar cell. However, as organic material generally, has lower bandgap $(<2 \mathrm{eV})$, Si/organic heterojunction solar cell as illustrated in Fig 5.1(a) suffers from parasitic optical absorption loss. The parasitic optical absorption issue is especially critical if we consider a heterojunction solar cell based on thin film $\mathrm{Si}$ where there is limited optical absorption in the Si active material. While the use of back junction, where the organic material is located at the back of $\mathrm{Si}$ and away from the incident light, can be used to overcome parasitic optical absorption problem [27], however, more complicated processes are required to produce robust high performing device.

incident light

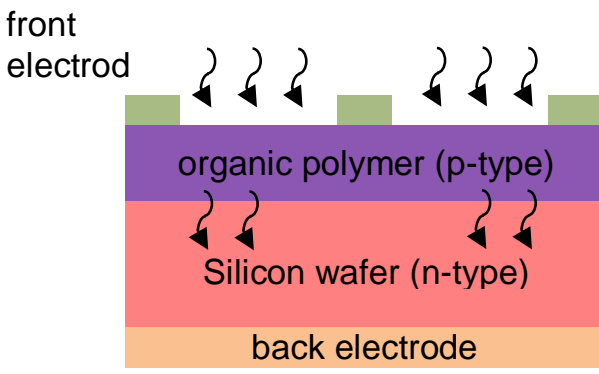

(a) Si/organic heterojunction incident light

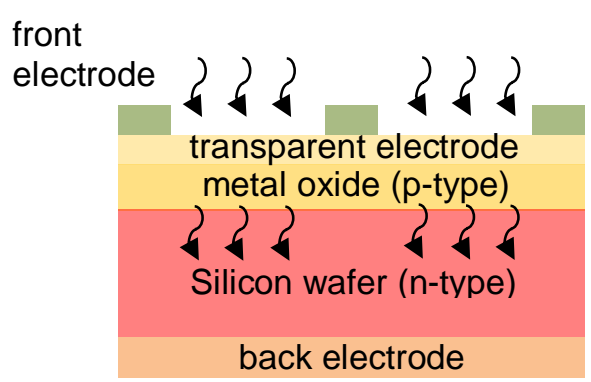

(b) Si/metal oxide heterojunction

Figure 5.1 : Schematics of (a) n-type Si/organic heterojunction solar cell and (b) ntype Si/metal oxide heterojunction solar cell. 
The motivation to achieve low-temperature fabrication process and low parasitic absorption loss has driven the development of Si/metal oxide heterojunction solar cell. The metal oxide can be deposited by various low temperature means, including thermal evaporation [97], atomic layer deposition [44] and solution based processing [45]. The thermal evaporation of metal oxide through shadow mask has been demonstrated to simplify fabrication of interdigitated back contact structure [42]. It is also potentially useful for the patterning process of another high-efficiency solar cell concept, such as localized rear contact and emitter wrap through design. Metal oxide exhibits large optical bandgap and allows high transparency which is crucial for simple front junction solar cell design as shown in Fig. 5.1(b). Such front junction design is still the most commonly used structure in commercial photovoltaic (PV) market due to its simple structure, lower cost fabrication process and its good efficiency. Besides, the design imposes less stringent requirement on the quality of the Si layer, as compared to other designs where the junction is located at the back. There are various metal oxides that can provide a strong hole or electron carrier selectivity when deposited on Si to form Si/Metal heterojunction. Hole accepting layers such as $\mathrm{V}_{2} \mathrm{O}_{x}[173]$, $\mathrm{WO}_{\times}[98,173]$ or $\mathrm{MoO}_{\times}[53,97,173]$ can be used with n-type $\mathrm{Si}$, and in particular $\mathrm{Si} / \mathrm{MoO}_{x}$ solar cell has been reported with $18.8 \%$ power conversion efficiency and open circuit voltage as high as $710 \mathrm{mV}$ [52]. Electron injection layer such as $\mathrm{TiO}_{\times}[95]$ has also been demonstrated to act as a back surface field for $\mathrm{n}$-type $\mathrm{Si}[95]$ and can be used as an emitter for $\mathrm{p}$-type $\mathrm{Si} / \mathrm{TiO}$ x solar cell. 
In the n-type Si/metal oxide structure as shown in Fig. 5.1(b), light is incident on top of the metal oxide layer. Since the metal oxide possesses large optical bandgap, light passes through the metal oxide layer and is absorbed in the Si active layer with minimum parasitic absorption loss in the metal oxide, in contrast to the n-type Si/organic heterostructure as shown in Fig. 5.1(a). Electron-hole pairs will be generated upon absorption in $\mathrm{Si}$, and the metal oxide will accept hole and repel electron to generate photocurrent. A transparent electrode layer such as ITO is deposited on top of the metal oxide to serve as an anti-reflective-coating (ARC) [54] and to assist majority carrier collection from the metal oxide [97]. It is expected that the simple planar Si/metal oxide structure as shown in Fig. 5.1(b) will produce larger current than the Si/organic hybrid solar cell shown in Fig. 5.1(a), thanks to the reduced parasitic absorption loss in the metal oxide as compared to the organic layer. This is particularly beneficial when thin film $\mathrm{Si}$ is used as the active layer to achieve low-cost, high-performance Si solar cell.

Research on $\mathrm{Si} /$ metal oxide heterojunction, especially on $\mathrm{Si} / \mathrm{MoO}_{x}$, is developing at a promising pace. C. Battaglia et al. fabricated hole selective $\mathrm{MoO}_{x}$ contact Si/MoOx heterojunction solar cell with a microtextured front junction, which exhibited an open circuit voltage of $580 \mathrm{mV}$ and efficiency of $14.3 \%$ [97]. Insertion of a-Si:H passivation layer between $\mathrm{Si}$ and $\mathrm{MoO}_{x}$ has been shown to improve the open circuit voltage to $711 \mathrm{mV}$ and the efficiency to $18.8 \%$ [52]. J. Bullock et al. recently demonstrated that $\mathrm{SiO}_{x}$ could also be used to provide passivation for $\mathrm{Si} / \mathrm{MoO}_{x}$ heterojunction solar cell, resulting in an open circuit voltage of $637 \mathrm{mV}$ [54]. 
Interestingly, a high short circuit current density of $35 \mathrm{~mA} / \mathrm{cm}^{2}$ and an efficiency of $16.7 \%$ has been achieved for planar front junction cell, demonstrating the excellent potential of wide bandgap $\mathrm{MoO}_{x}$ as a transparent hole injection layer [54]. Junctionless front surface nanohole was employed by Um et al. [42], coupled with interdigitated back contact structure with planar $\mathrm{Si} / \mathrm{MoO}_{x}$ interface at the back, to provide additional light trapping. A current density of $37 \mathrm{~mA} / \mathrm{cm}^{2}$ and efficiency of $15.4 \%$ have been reported for the structure [42]. The lower efficiency obtained as compared to that of J. Bullock et al. [54] is due to the lower open circuit voltage that might be attributed to unoptimized rear contact geometry employed [42] or a lack of proper passivation for the front surface nanohole.

\section{$5.2 \mathrm{Si} /$ molybdenum oxide heterojunction solar cell}

Silicon/transition metal oxide (Si/TMO) heterojunction solar cells based on $\mathrm{n}$ $\mathrm{Si} /$ molybdenum oxide $\left(\mathrm{n}-\mathrm{Si} / \mathrm{MoO}_{\mathrm{x}}\right)$ structure have been recently reported with high power conversion efficiency (PCE) of 14\% [97] and 18\% [52] respectively for unpassivated and PECVD passivated devices. The un-passivated and PECVD passivated devices demonstrated open circuit voltage $\left(\mathrm{V}_{\text {oc }}\right)$ of $580 \mathrm{mV}$ [97] and 711 $\mathrm{mV}$ [97], respectively, revealing the excellent selective carrier contact property of $\mathrm{MoO}_{x}$ on $\mathrm{n}-\mathrm{Si}$. It is desirable to achieve high $\mathrm{V}_{\mathrm{oc}}$ without resorting to PECVD passivation process which might limit the process throughput and the practical application of the solar cell. Alternative deposition methods such as evaporation or solution based processing will be more attractive for commercial applications. 
In practice, evaporated molybdenum oxide forms an oxygen deficient $\mathrm{MoO}_{x}$ structure with $x<3$. The oxygen to molybdenum atomic ratio $x$ in the compound has been reported to be in the range of $2.4<x<2.9$ [174]. $\mathrm{MoO}_{\mathrm{x}}$ is known to be very sensitive to moisture and oxygen in ambient air and hence displays high variability in its work function. Irfan et al. found that for a $5 \mathrm{~nm}$ thin film of $\mathrm{MoO}_{\mathrm{x}}$, its work function is reduced from $6.8 \mathrm{eV}$ to $5.3 \mathrm{eV}$ when exposed to air, while saturates at $5.7 \mathrm{eV}$ when exposed to pure oxygen [175], and can recover partially to $6.3 \mathrm{eV}$ upon a desorption process [174]. The work function of $\mathrm{MoO}_{\mathrm{x}}$ is reduced more in the air than in oxygen, which is attributed to adsorption of water moisture. Photoemitted electron spectroscopy revealed that the surface of $\mathrm{MoO}_{x}$ film is significantly more oxygen-rich $(x=2.8-3.1)$ than the bulk $(x=2.4-2.5)$, and film exposed to air has a significantly higher surface concentration of oxygen $(x=2.95-3.1)$ as compared to as-evaporated or annealed film $(x=2.8-2.9)$ [174].

$\mathrm{MoO}_{\mathrm{x}}$ is often incorrectly assumed to be a p-type semiconductor since it was previously known to assist in hole injection and extraction for various organic-based devices [94]. However, a later study using UPS and IPES spectra revealed that $\mathrm{MoO}_{\mathrm{x}}$ is an $\mathrm{n}$-type semiconductor with an electron affinity of $6.7 \mathrm{eV}$, a work function of $6.9 \mathrm{eV}$, ionization energy of $9.7 \mathrm{eV}$, and a bandgap of $3.0 \mathrm{eV}$ [94]. The band offsets between $\mathrm{Si}$ and $\mathrm{MoO}_{\mathrm{x}}$ is not well studied on literature. The mechanism of $\mathrm{MoO}_{x}$ carrier selectivity has been previously studied on heterojunction structures involving $\mathrm{MoO}_{x}$ interface with an organic semiconducting material such as 4,4'-Bis( $\mathrm{N}$-carbazolyl)-1,1'-bipheny (CBP). Figure 5.2 (left) shows that the high work 
function of $\mathrm{MoO}_{\mathrm{x}}$ induces strong band bending in $\mathrm{CBP}$, which allows a small energy gap to develop between the highest occupied molecular orbital (HOMO) level of CBP and the conduction band (CB) of $\mathrm{MoO}_{x}$ [94]. The small energy gap facilitates hole injection by electron transfer from the $\mathrm{HOMO}$ of $\mathrm{CBP}$ to the $\mathrm{CB}$ of $\mathrm{MoO}_{x}$, while the hole extraction is through a transfer of an electron from the $\mathrm{CB}$ of $\mathrm{MoO}_{\mathrm{x}}$ to the $\mathrm{HOMO}$ of $\mathrm{CBP}$, as seen in Fig. 5.2 (right). This mechanism allows $\mathrm{MoO}_{\mathrm{x}}$ to inject or extract holes, although it mainly conducts electrons. On the other hand, the electron-blocking property of $\mathrm{MoO}_{x}$ does not result from the material itself, but by the induced energy level bending on the LUMO of the CBP.
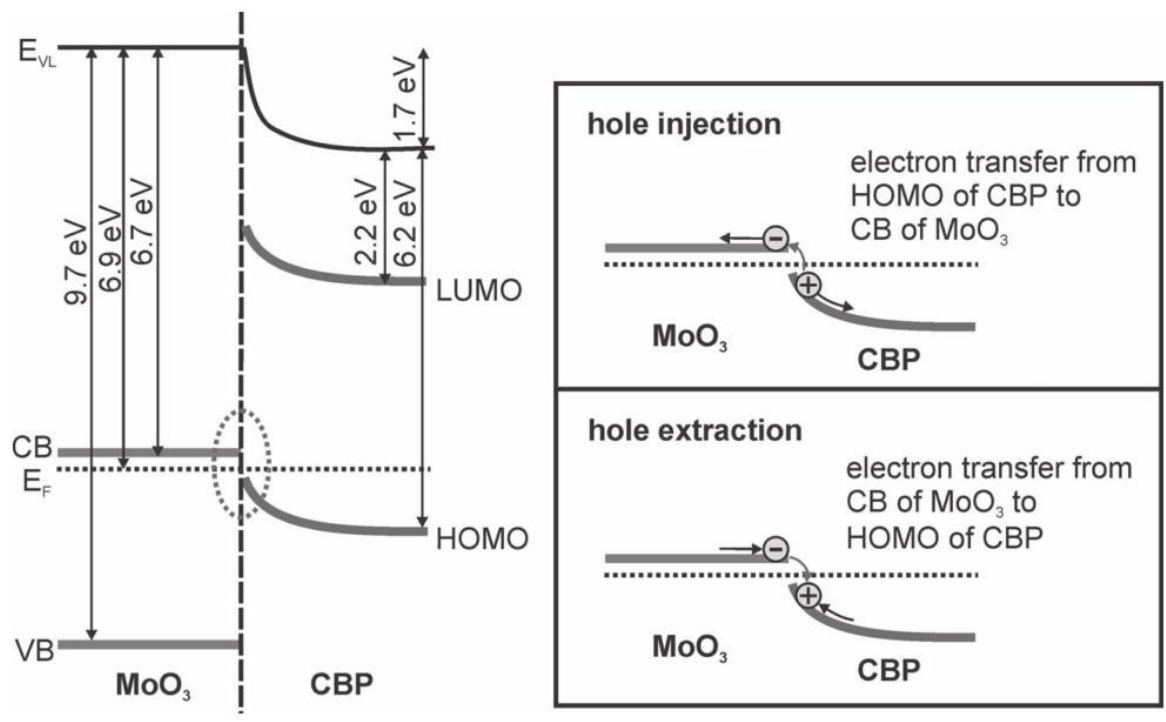

Figure 5.2 : Energy level alignment of $\mathrm{MoO}_{x} / \mathrm{CBP}$ interface (left) and the model for hole injection and the transfer mechanism (right) [174]. 


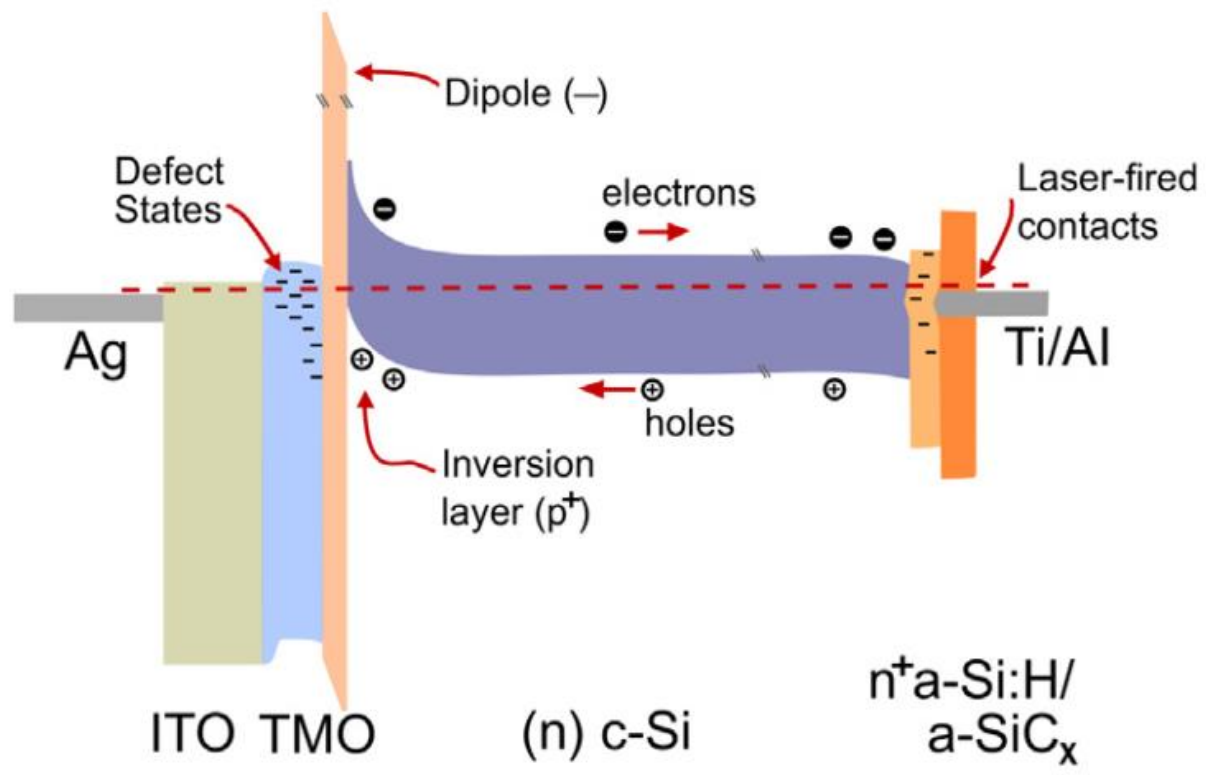

Figure 5.3 : A model of Si/TMO heterojunction solar cell based on n-type TMO with an electron blocking property [99].

Due to its unusually high work function, $\mathrm{MoO}_{x}$ can also induce inversion layer in $\mathrm{n}$ $\mathrm{Si}[52,99]$ to allow hole extraction while blocking electron, thus achieving small dark recombination current. A model of Si/TMO heterojunction solar cell energy level alignment based on $\mathrm{MoO}_{x}$ material is proposed by Gerling et al. [99] as seen in Fig 5.3. In this case, hole collection will be equivalent to a transfer of an electron from the TMO to the hole in the Si inversion layer $\left(\mathrm{p}^{+}\right)$, while the electron blocking is provided by the Schottky barrier. Gerling et al. also proposed the formation of a negative dipole on the $\mathrm{TMO} / \mathrm{n}$-Si interface due to trapped negative charges which contribute to electron blocking. 
In summary, molybdenum oxide $\left(\mathrm{MoO}_{\mathrm{x}}\right)$ has a high value of work function, but it may vary widely due to oxygen or moisture adsorption (5.3 eV to $6.8 \mathrm{eV}$ ) after the evaporation process. Since $\mathrm{MoO}_{\mathrm{x}}$ is an $\mathrm{n}$-type semiconductor, the electron blocking capability of the $\mathrm{n}-\mathrm{Si} / \mathrm{MoO}_{x}$ heterojunction is solely a result of the high band bending in the conduction band of the $\mathrm{n}$-Si. Since the band bending is reliant on the high work function of $\mathrm{MoO}_{x}$, it is important to ensure high $\mathrm{MoO}_{x}$ material quality in forming the heterojunction, so that it can provide strong carrier selectivity to n-Si. The interface passivation between $\mathrm{n}-\mathrm{Si}$ and $\mathrm{MoO}_{\mathrm{x}}$ is another important consideration to minimize minority carrier recombination as well as to manipulate dipole formation to increase electron barrier. In addition, the Si substrate doping concentration has to be tuned to control the Si Fermi level, so as to produce favorable energy band alignment.

\subsection{Fabrication and optimization of Si/MoOx heterojunction solar cell}

\subsubsection{Fabrication process}

In this section, we present our study on the fabrication of $n-\mathrm{Si} / \mathrm{MoO}_{x}$ heterojunction solar cell by e-beam evaporation of $\mathrm{MoO}_{3}$ on $\mathrm{Si}$. The influence of the $\mathrm{Si}$ doping level $\left(N_{D}\right)$, the use of PEDOT:PSS and ITO electrode, the incorporation of a water barrier layer on $\mathrm{MoO}_{x}$, and the native oxide passivation of the $\mathrm{n}-\mathrm{Si} / \mathrm{MoO}$ interface on the

photovoltaic performance of $\mathrm{n}-\mathrm{Si} / \mathrm{MoO}_{\mathrm{x}}$ heterojunction solar cell will be investigated. 
(a)

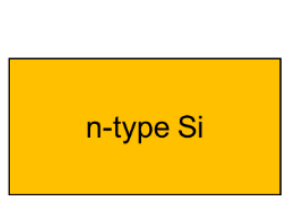

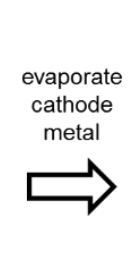

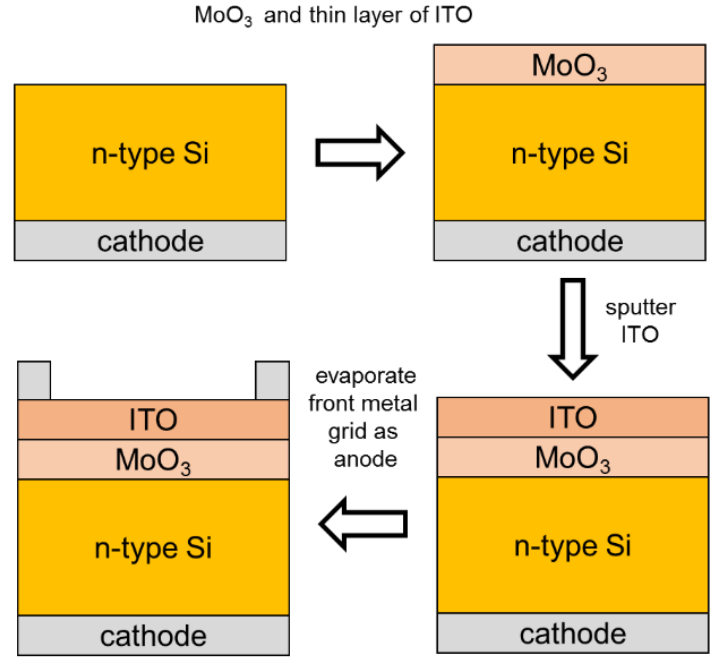

evaporate

$\mathrm{MoO}_{3}$ and thin layer of ITO (b)

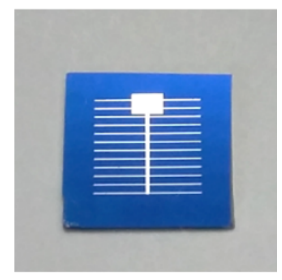

Figure 5.4 : (a) Fabrication process of baseline $\mathrm{n}-\mathrm{Si} / \mathrm{MoO}_{\mathrm{x}}$ heterojunction solar cell and (b) optical image of $\mathrm{n}-\mathrm{Si} / \mathrm{MoO}_{x}$ solar cell.

Fig. 5.4 shows the fabrication process of baseline $n-\mathrm{Si} / \mathrm{MoO}_{x}$ heterojunction solar cell. The starting $\mathrm{n}-\mathrm{Si}<100>\mathrm{CZ}$ wafers with a thickness of $675 \mu \mathrm{m}$ were cleaned with acetone, IPA, and DI water, before subsequently immersed in dilute HF solution to remove native oxide. Cathode layer based on $\mathrm{Ti}(20 \mathrm{~nm}) / \mathrm{Pd}(20 \mathrm{~nm}) / \mathrm{Ag}$ (200 nm) was evaporated on the back side with Edward EB3 e-beam evaporation machine. A $15 \mathrm{~nm}$ of $\mathrm{MoO}_{x}$ layer was evaporated from stoichiometric $\mathrm{MoO}_{3}$ source using the same e-beam evaporation machine at a rate of $0.05-0.1 \mathrm{~nm} / \mathrm{s}$ and a chamber pressure of $10^{-6}$ to $10^{-5}$ mbar. For the solar cells having ITO electrode, the samples were transferred through the air to a Denton RF sputtering machine to sputter $60 \mathrm{~nm}$ of ITO at a rate of $10 \mathrm{~nm} / \mathrm{min}$, using Argon at a flow rate of $50 \mathrm{sccm}$ subjected to $100 \mathrm{~W}$ RF power. The samples were then annealed at $150^{\circ} \mathrm{C}$ in an $\mathrm{N}_{2}$ atmosphere. For the characterization of the photovoltaic parameters, the samples were illuminated using San-El solar simulator system with the AM1.5 reference 
spectrum, calibrated with Si solar cell calibration standard. The dark I-V parameters were also measured using the same setup. The optical image of $n-S i / M o O_{x}$ solar cell is shown in Fig. 5.4(b).

\subsubsection{Si/MoOx solar cell with various Si substrate doping}

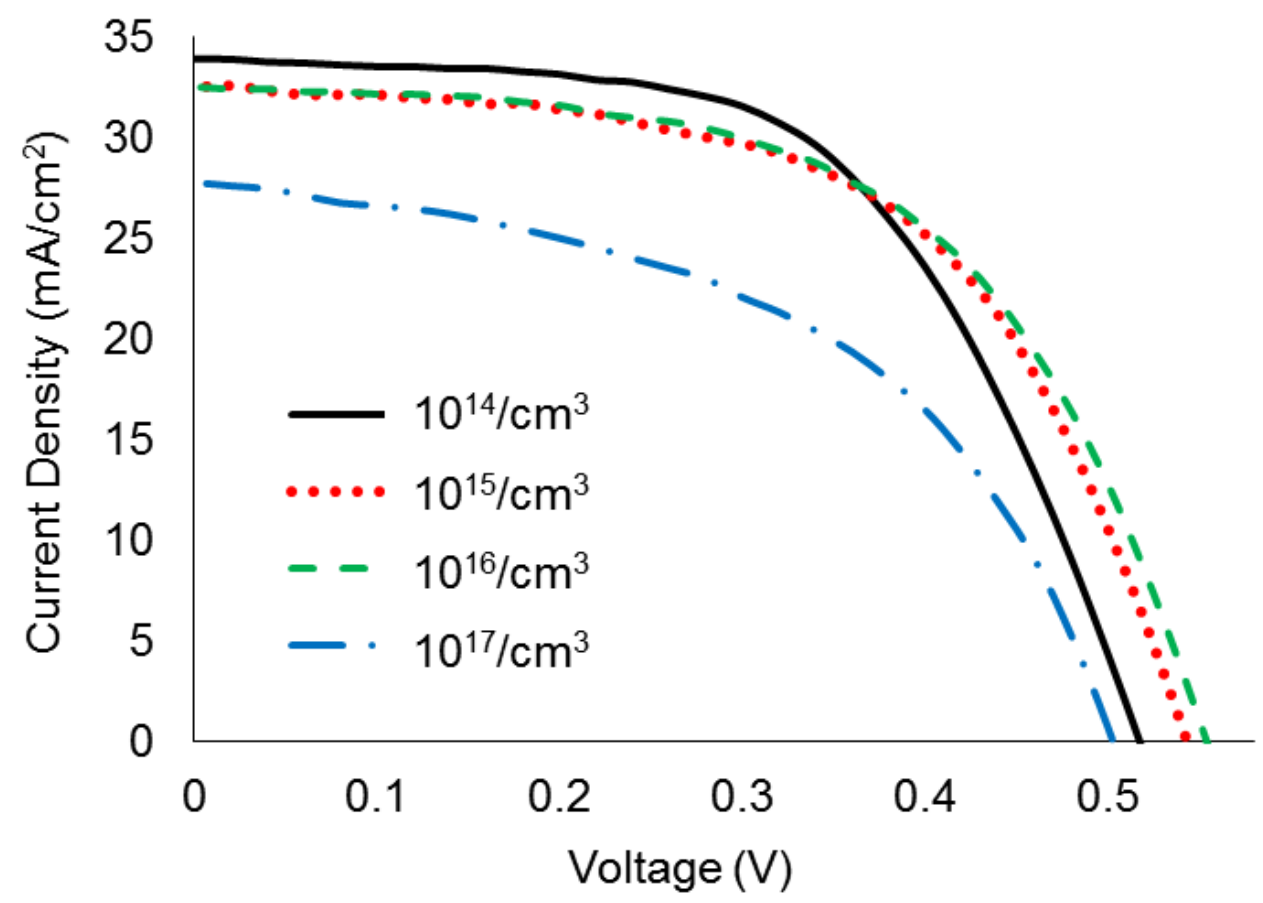

Figure 5.5 : Illuminated J-V curve of $\mathrm{n}-\mathrm{Si} / \mathrm{MoO}_{x} / \mathrm{ITO}$ solar cell with various $\mathrm{n}-\mathrm{Si}$ substrate doping concentrations.

Figure 5.5 shows the illuminated $\mathrm{J}-\mathrm{V}$ characteristics of $\mathrm{n}-\mathrm{Si} / \mathrm{MoO}_{\times}$based solar cells with ITO electrodes at various $\mathrm{n}$-Si doping concentrations $N_{\mathrm{D}}$, and the photovoltaic parameters are summarized in Table 5.1. For $N_{D}=10^{14}-10^{16} / \mathrm{cm}^{3}$ the short circuit current densities $J_{\mathrm{sc}}$ as shown in Table 5.1 are relatively high at $32.5-33.9 \mathrm{~mA} / \mathrm{cm}^{2}$ which is almost close to that of the planar device reported by Bullock et al. [54] at $35 \mathrm{~mA} / \mathrm{cm}^{2}$. As $N_{D}$ increases to $10^{17} / \mathrm{cm}^{3}$, the $J_{\text {sc }}$ decreases to $27.7 \mathrm{~mA} / \mathrm{cm}^{2}$ due to 
the lower carrier diffusion length in Si under higher doping concentration. It is found that $V_{o c}$ increases with $N_{D}$ from 0.518 to $0.554 \mathrm{~V}$ over the range $N_{D}=10^{14}-10^{16}$ $/ \mathrm{cm}^{3}$, which is typically attributed to reduced dark saturation current. At higher $N_{D}=$ $10^{17} / \mathrm{cm}^{3}, V_{\mathrm{oc}}$ decreases to $0.503 \mathrm{~V}$. On the other hand, the FF monotonically decreases from 57.5 to $49.9 \%$ with increasing $N_{D}$, which is not expected since $V_{o c}$ increases with $N_{D}$ over the range of $10^{14}-10^{16} / \mathrm{cm}^{3}$, and the bulk wafer series resistance is lower at higher doping concentration. The PCE is observed to be almost independent of doping at 10.1 to $10.2 \%$ for $N_{D}=10^{14}-10^{16} / \mathrm{cm}^{3}$, and it drops to $6.9 \%$ at $N_{\mathrm{D}}=10^{17} / \mathrm{cm}^{3}$.

Table $5.1: \mathrm{n}$-Si/MoOx/ITO solar cell device as a function of $\mathrm{n}$-Si donor concentration.

\begin{tabular}{|c|c|c|c|c|}
\hline $\begin{array}{l}\mathrm{N}_{\mathrm{D}} \\
\left(/ \mathrm{cm}^{3}\right)\end{array}$ & $\begin{array}{l}\mathrm{J}_{\mathrm{sc}} \\
\left(\mathrm{mA} / \mathrm{cm}^{2}\right)\end{array}$ & $\begin{array}{l}\mathrm{V}_{\mathrm{oc}} \\
(\mathrm{V})\end{array}$ & $\begin{array}{l}\text { FF } \\
(\%)\end{array}$ & $\begin{array}{l}\text { PCE } \\
(\%)\end{array}$ \\
\hline $10^{14}$ & 33.9 & 0.518 & 57.5 & 10.1 \\
\hline $10^{15}$ & 32.5 & 0.543 & 57.2 & 10.1 \\
\hline $10^{16}$ & 32.5 & 0.554 & 56.6 & 10.2 \\
\hline $10^{17}$ & 27.7 & 0.503 & 49.9 & 6.9 \\
\hline
\end{tabular}

We further investigate the dark $\ln ([J]-V)$ as seen in Fig. 5.6. We found that at a low voltage bias, all devices reveal a significant ohmic component of shunt leakage as can be seen by the relatively symmetrical $\ln ([\mathrm{J}] \mathrm{V})$ curve at a voltage range of -0.2 
to $0.2 \mathrm{~V}$. The dark current increases from $N_{\mathrm{D}}=10^{14} / \mathrm{cm}^{3}$ to $10^{15} / \mathrm{cm}^{3}$ especially at lower forward bias voltage range. The dark current for cells with $N_{D}$ of $10^{15}$ and $10^{16}$ $/ \mathrm{cm}^{3}$ is almost similar over the entire voltage range. At forward bias voltage greater than $0.5 \mathrm{~V}$, all the dark current curves for $N_{\mathrm{D}}=10^{14}-10^{16} / \mathrm{cm}^{3}$ converge. For $N_{\mathrm{D}}=$ $10^{17} / \mathrm{cm}^{3}$, the dark current is significantly larger than those at $N_{D}=10^{16} / \mathrm{cm}^{3}$, which is consistent with the observed drop in $V_{\text {oc. }}$. As previously shown, we found that the $V_{o c}$ increase with $N_{D}$ at $N_{D}=10^{14}-10^{16} / \mathrm{cm}^{3}$. On the other hand, the dark current increase with $N_{D}$ at $N_{D}=10^{14}-10^{17} / \mathrm{cm}^{3}$. Since the increase on $V_{o c}$ is not caused by the decrease in dark current, the device junction behaviour is not consistent as expected of a conventional $p-n$ junction solar cell. For a $p-n$ junction solar cell, we expect that the $V_{o c}$ increase with $N_{D}$ due to reduced dark current. Further characterization work is needed to understand the junction behaviour of $n-\mathrm{Si} / \mathrm{MoO}_{x}$ solar cell. We also found an increased component of ohmic shunt leakage with increased $N_{D}$, which is also correlated with the reduced FF when $N_{D}$ increases from $10^{14}$ to $10^{17} / \mathrm{cm}^{3}$. 


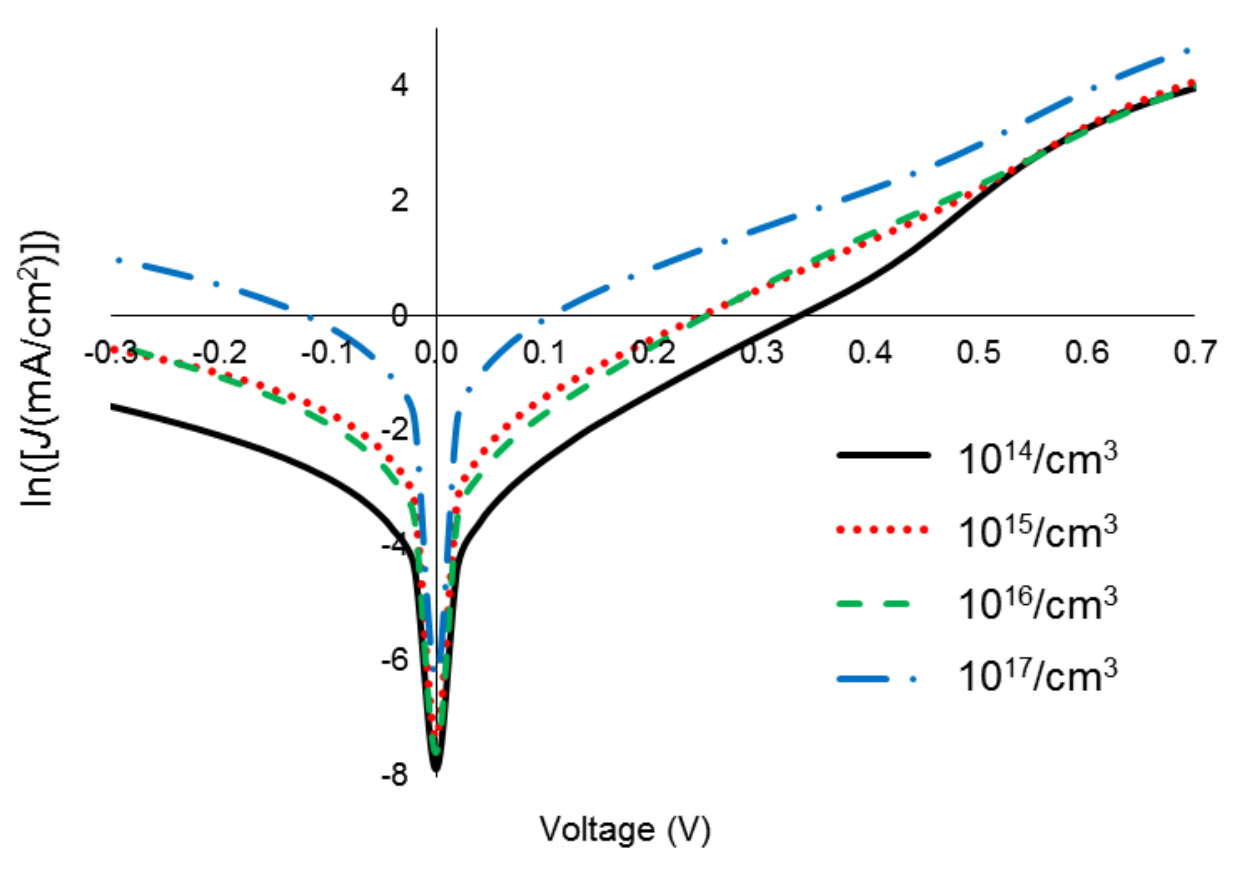

Figure 5.6 : Effect of $N_{D}$ on the dark $J-V$ curve of $n-S i / M o O x$ based solar cell.

\subsubsection{Si/MoOx solar cell with PEDOT:PSS electrode}

In this section, we study the use of PEDOT:PSS instead of ITO as an electrode for Si/MoOx solar cell. PEDOT:PSS has a work function of $\approx 5.2 \mathrm{eV}$, which is higher than ITO of $\approx 4.8 \mathrm{eV}[176]$. The higher work function of PEDOT:PSS may induce favorable electronic level alignment with $\mathrm{MoO}_{x}$ which also has a relatively high work function of $\approx 5.3-6.8 \mathrm{eV}[174,175]$. This may allow us to realize higher open circuit voltage $V_{o c}$ and FF. Besides, PEDOT:PSS is easier to deposit as compared to sputtered ITO, which thus leads to a simpler fabrication process. 
(a) $\mathrm{Si} / \mathrm{MOO}_{\mathrm{x}} / \mathrm{PEDOT}: \mathrm{PSS}$ without barrier layer

\begin{tabular}{|c|}
\hline $\mathrm{MoO}_{\mathrm{x}}$ \\
\hline n-type Si \\
\hline cathode \\
\hline
\end{tabular}
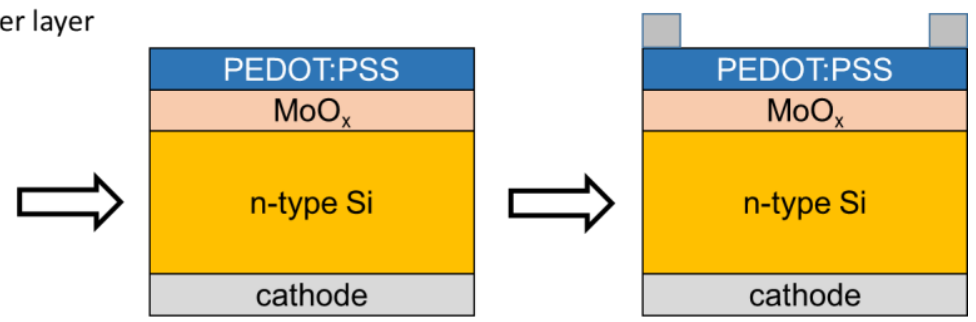

(b) $\mathrm{Si} / \mathrm{MOO}_{\mathrm{x}} / \mathrm{PEDOT}: \mathrm{PSS}$ with thin ITO barrier layer
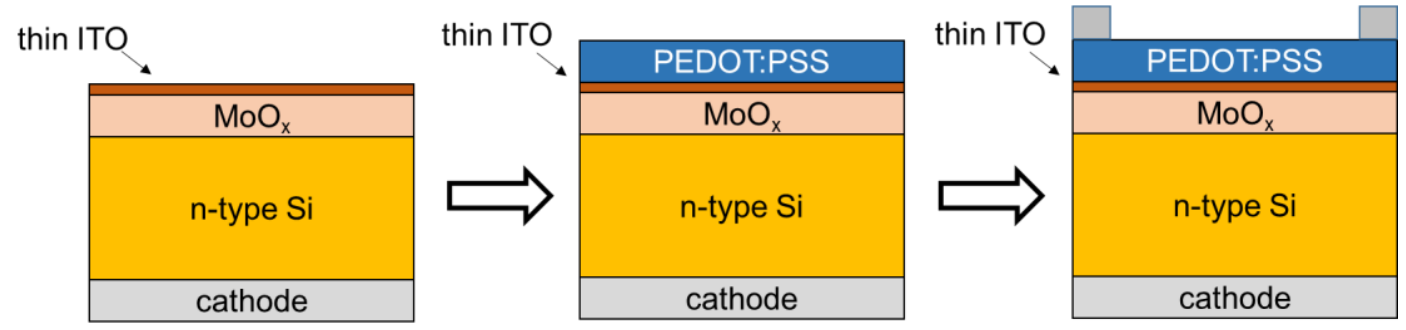

Figure 5.7 : Fabrication process of $\mathrm{Si} / \mathrm{MoO}_{x}$ heterojunction solar cell with PEDOT:PSS as an electrode. (a) Direct contact between $\mathrm{MoO}_{x}$ and PEDOT:PSS and (b) thin evaporated ITO layer incorporated between $\mathrm{MoO}_{x}$ and PEDOT:PSS.

Figure 5.7 shows the fabrication process of $\mathrm{Si} / \mathrm{MoO}_{x}$ heterojunction solar cell with PEDOT:PSS as an electrode. Cathode/n-type Si/MoO $\times$ structure was fabricated as previously described in Fig. 5.4. To minimize adsorption of water on $\mathrm{MoO}_{x}$ arising from the PEDOT:PSS coating process, we also fabricated such solar cell with a 5 nm thin layer of evaporated ITO as shown in Fig. 5.7(b) to serve as a water barrier between $\mathrm{MoO}_{x}$ and PEDOT:PSS. We choose ITO as a water barrier layer since it is known to make good electrical contact with PEDOT:PSS [176] and is water resistant [177]. Note that the evaporation process, instead of sputtering process, is used so that the ITO can be evaporated as a water barrier directly after $\mathrm{MoO}_{\mathrm{x}}$ evaporation in the same vacuum without exposure to air. The solar cells with the 
thin ITO layer were subsequently annealed in two different conditions at $150^{\circ} \mathrm{C}$ for 10 min (i) in $\mathrm{N}_{2}$ ambient and (ii) under UV illumination in ozone ambient (UV-ozone treatment). After the treatment, PEDOT:PSS water based solution with 5 wt $\%$ DMSO and 1 wt $\%$ Triton $X-100$ was spin coated on top of the thin ITO layer, followed by $10 \mathrm{~min}$ annealing at $105^{\circ} \mathrm{C}$ on a hotplate to dry the PEDOT:PSS layer. Anodic finger grid was evaporated on top of the PEDOT:PSS layer as the top contact to form both $\mathrm{Si} / \mathrm{MoO}_{x} / \mathrm{PEDOT}: \mathrm{PSS}$ and $\mathrm{Si} / \mathrm{MoO}_{x} / \mathrm{ITO} / \mathrm{PEDOT}: \mathrm{PSS}$ solar cells as shown in Fig. 5.7.

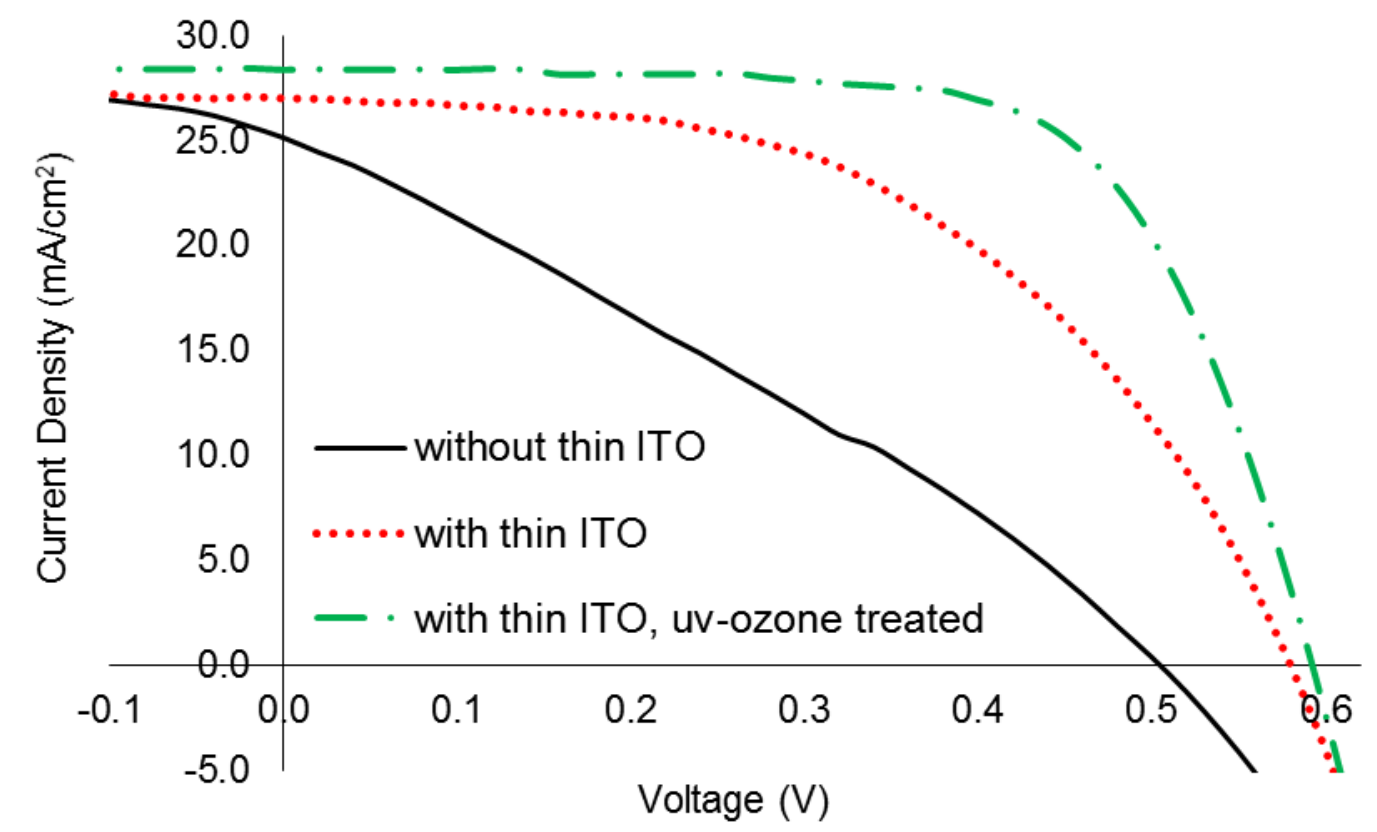

Figure 5.8 : Illuminated $J-V$ curves of $n-S i / M_{\rtimes} /$ PEDOT:PSS solar cell and $n$ Si/MoO^/ITO/PEDOT:PSS solar cells with different treatment conditions.

Figure 5.8 shows illuminated the $J-V$ curves of $n-S_{i} / M_{0}{ }_{x} / P E D O T: P S S$ solar cell and $\mathrm{n}-\mathrm{Si} / \mathrm{MoO}_{\star} /$ ITO/PEDOT:PSS solar cell annealed under $\mathrm{N}_{2}$ and UV illumination and ozone ambient. The solar cell without the ITO barrier layer exhibits triangular- 
like $J-V$ characteristic, indicating a weak rectifying property. In contrast, the solar cell with ITO barrier layer reveals more rectangle-like shaped $J-V$ characteristic that is more typical of a good solar cell. As compared to the cell annealed in $\mathrm{N}_{2}$ ambient, the cell treated with UV-ozone exhibits $J$ - $V$ curve that is closer to ideal photovoltaic response, with a small slope at $V=0$ and an almost vertical $J$ - $V$ curve near $V_{o c}$. Table 5.2 shows the photovoltaic parameters of the $n$-Si/MoO^/PEDOT:PSS solar cells with various conditions of the barrier layer. It is seen that the FF increases from 28.6 to $50.7 \%$ and $V_{o c}$ increases from 0.505 to $0.579 \mathrm{~V}$ with the introduction of the thin ITO water barrier layer annealed in a nitrogen ambient. Water adsorption significantly reduces the work function of $\mathrm{MoO}_{x}$ [175], which in turn compromises the rectifying property of the $n-S i / M_{0} O_{x}$ junction. This is believed to be the cause of the lower $V_{o c}$, and FF observed for the n-Si/MoOx/PEDOT:PSS solar cell without the thin ITO water barrier layer. Hence, the higher $V_{o c}$ for a device with the barrier layer may have been caused by the preservation of $\mathrm{MoO}_{\mathrm{x}}$ work function which is crucial for the electron blocking properties of $n-S i / M_{0} O_{x}$ junction. When the ITO layer was subjected to UV-ozone treatment, the FF and $V_{o c}$ are further improved to $67.3 \%$ and $0.592 \mathrm{~V}$ respectively. This is attributed to the fact that UV-ozone treatment improves the wettability of ITO [177], which will result in a more uniform and better contact formed between the ITO layer and the PEDOT:PSS layer. This will lower the series resistance of the solar cell and result in a higher FF. We also noted that $J_{\mathrm{sc}}$ is observed to increase from $25.1 \mathrm{~mA} / \mathrm{cm}^{2}$ to $27.0 \mathrm{~mA} / \mathrm{cm}^{2}$ when the barrier layer is introduced, and further enhanced to $28.4 \mathrm{~mA} / \mathrm{cm}^{2}$ when the UVozone treatment is applied. The improvement in the photovoltaic parameters has 
resulted in a high PCE of $11.3 \%$ achieved for the cell incorporated with thin ITO water barrier layer treated with UV-ozone.

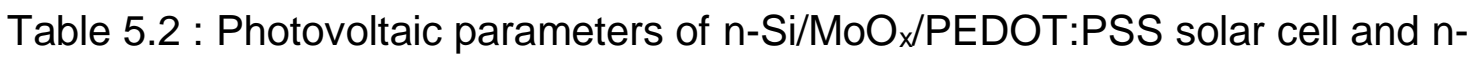
Si/MoO^/ITO/PEDOT:PSS solar cells with different treatment conditions.

\begin{tabular}{|l|c|c|c|c|}
\cline { 2 - 5 } \multicolumn{1}{c|}{} & \multicolumn{4}{c|}{ Photovoltaic Parameters } \\
\hline Sample & JsC & Voc & FF & PCE \\
& $\left(\mathrm{mA} / \mathrm{cm}^{2}\right)$ & (V) & $(\%)$ & $(\%)$ \\
\hline Without ITO & 25.1 & 0.505 & 28.6 & 3.6 \\
\hline With thin ITO & & & & \\
annealed in N2 & 27.0 & 0.579 & 50.7 & 7.9 \\
\hline With thin ITO & & & & \\
annealed in UV- & & & & \\
ozone & 28.4 & 0.592 & 67.3 & 11.3 \\
\hline
\end{tabular}

Figure 5.9 shows the dark $J-V$ characteristics of $n-S i / M_{x}{ }_{\rtimes} /$ PEDOT:PSS solar cell and $\mathrm{n}-\mathrm{Si} / \mathrm{MoO}_{x} / \mathrm{ITO} / \mathrm{PEDOT}: \mathrm{PSS}$ solar cells with different treatment conditions. In general, they present a consistent picture of reduced dark saturation current with the introduction of the thin ITO layer with the UV-ozone treatment, which in turn leads to an increase in $V_{\text {oc. }}$ We believe that the major cause of high saturation current for the solar cell without the water barrier layer is excessive water moisture adsorbance that is caused by the PEDOT:PSS deposition process. The introduction of the thin ITO layer as water barrier avoids water-induced defects 
[175] and preserves the $\mathrm{MoO}_{x}$ work function, resulting in the $n$ Si/MoO $/$ PEDOT:PSS solar cell with relatively high $V_{o c}$ of $0.579 \mathrm{~V}$. Besides increasing the wettability of PEDOT:PSS on ITO [177], the use of UV-ozone treatment may also increase the ITO work function [178], which helps provide a favorable band alignment with $\mathrm{MoO}_{\mathrm{x}}$, and in turn increase the Schottky barrier height and open circuit voltage of the solar cell.

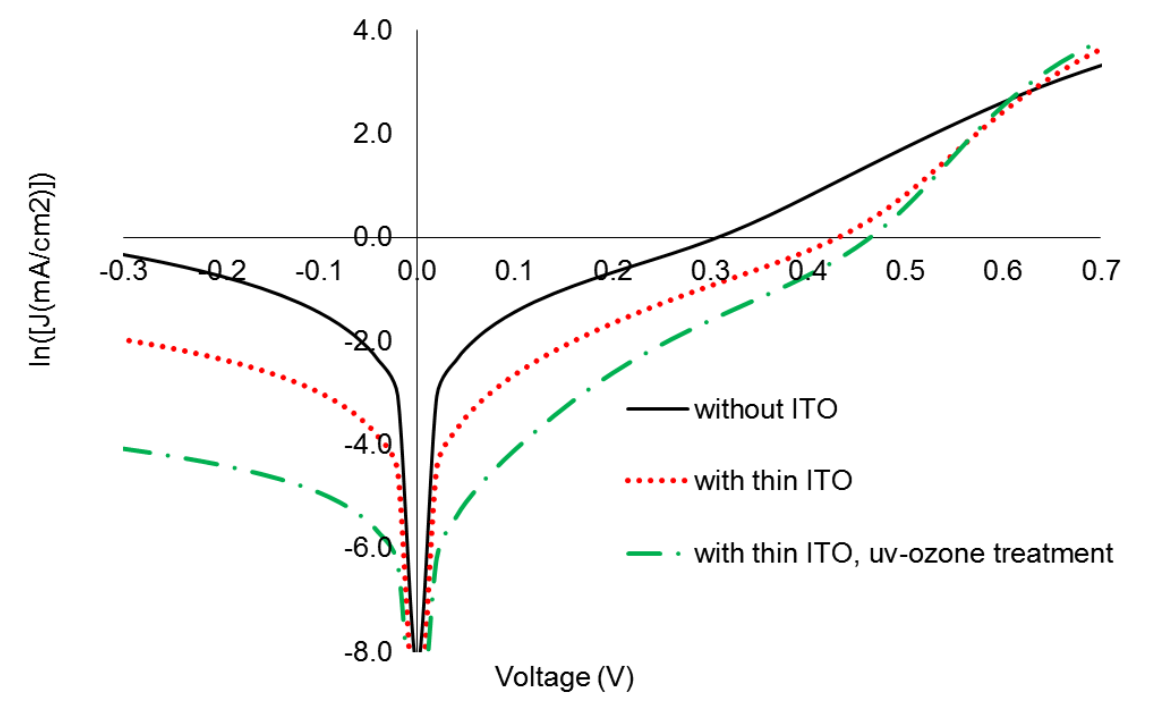

Figure 5.9 : Dark J-V curves of $n-S_{1} / M_{0}{ }_{x} /$ PEDOT:PSS solar cell and nSi/MoOx/ITO/PEDOT:PSS solar cells with different treatment conditions.

\subsubsection{Si/MoOx solar cell with ITO electrode}

Our earlier study of $\mathrm{n}-\mathrm{Si} / \mathrm{MoO}_{x} / \mathrm{ITO}$ solar cell with ITO electrode may suffer from water adsorption problem since the samples were exposed to air when they were transferred from the evaporation chamber after the growth of $\mathrm{MoO}_{\mathrm{x}}$ to the sputtering chamber for the deposition of ITO. Note that although the same evaporation 
chamber can be used to evaporate ITO as the electrode for the solar cell immediately after the evaporation of $\mathrm{MoO}_{x}$, however, this was not done as the evaporated ITO has much poorer quality than the sputtered ITO. From the result obtained for the n-Si/MoO^/PEDOT:PSS solar cell with PEDOT:PSS electrode that reveals the importance of the thin ITO barrier layer, it suggests that we can similarly introduce a thin layer of evaporated ITO to serve as a water barrier layer for the n$\mathrm{Si} / \mathrm{MOO}_{x} / \mathrm{ITO}$ solar cell, before the cells are transferred to the sputtering machine for ITO electrode deposition. Comparing the $n-S i / M o O x$ solar cells with PEDOT:PSS and ITO electrodes, it should be noted that the former suffers from parasitic absorption in PEDOT:PSS, and hence using ITO electrode may potentially improve its performance. Therefore, in this section, we investigate $\mathrm{n}-\mathrm{Si} / \mathrm{MoO}_{x} / \mathrm{ITO}$ solar cell incorporated with a thin water barrier layer of evaporated ITO on top of the $\mathrm{MoO}_{\mathrm{x}}$. For comparison purpose, we studied two different cell structures, one without thin ITO barrier layer as shown in Fig. 5.10(a), and the other one with an ITO barrier layer incorporated as shown in Fig. 5.10(b). The barrier layer was based on $5 \mathrm{~nm}$ evaporated thin ITO layer, which was deposited immediately in a vacuum after the evaporation of $\mathrm{MoO}_{\mathrm{x}}$. The layer was kept thin in order to minimize its impact on the overall transparency and conductivity of the solar cell.

For the fabrication process, Si substrates were cleaned in acetone, IPA and DI water in an ultrasonic bath, followed by a dip in $5 \% \mathrm{HF}$ solution to remove native oxide. Ti $(20 \mathrm{~nm}) / \mathrm{Pd}(20 \mathrm{~nm}) / \mathrm{Ag}(200 \mathrm{~nm})$ layer was evaporated on the back side as a cathode in an Edward EB3 e-beam evaporation machine in $10^{-5}-10^{-6}$ mbar 
range. $\mathrm{MoO}_{x}$ layer was evaporated on the top side using the same machine at a rate of $0.05-0.1 \mathrm{~nm} / \mathrm{s}$ in $10^{-5}-10^{-6} \mathrm{mbar}$ range. For the device with the barrier layer, a thin $(5 \mathrm{~nm})$ ITO layer was evaporated directly on top of $\mathrm{MoO}_{x}$ layer in the same vacuum. The samples were transferred through ambient air to a Denton RF sputtering machine. A $60 \mathrm{~nm}$ thick ITO layer was sputtered at a rate of $10 \mathrm{~nm} / \mathrm{min}$, using Argon at a flow rate of $50 \mathrm{sccm}$ subjected to $100 \mathrm{~W}$ RF power. The entire samples were then annealed at $150^{\circ} \mathrm{C}$ in $\mathrm{N}_{2}$ ambient for 10 min before evaporated with Ag grid as the anode.

(a)

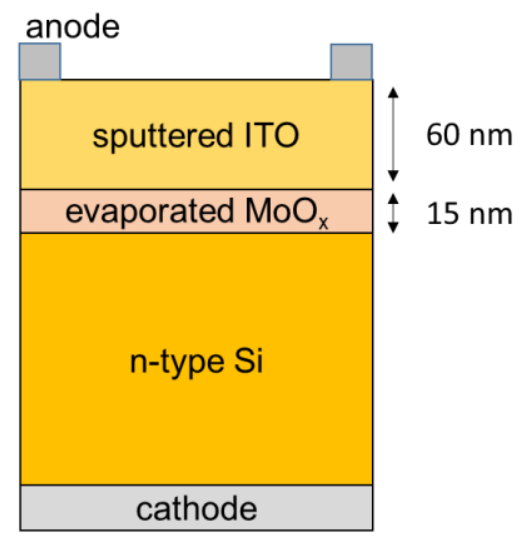

Major fabrication steps:

1. Solvent cleaning \& native oxide removal

2. Evaporation of cathode

3. Evaporation of $\mathrm{MoO}_{\mathrm{x}}(15 \mathrm{~nm})$

4. Sputtering of ITO $(60 \mathrm{~nm})$

5. Annealing at $\mathrm{N}_{2}$ ambient

6. Evaporation of anode (b)

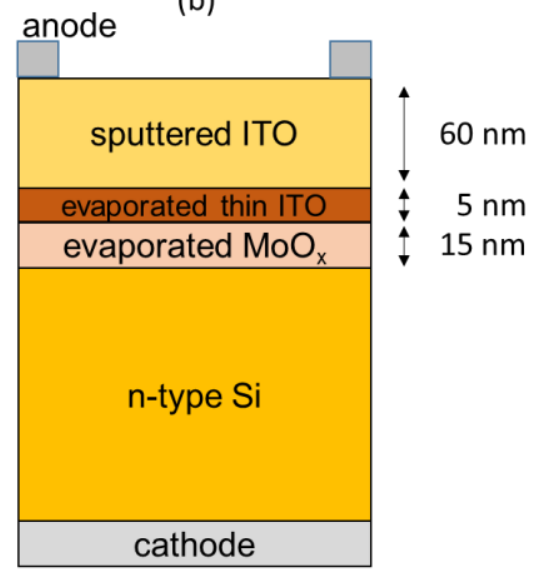

Major fabrication steps:

1. Solvent cleaning \& native oxide removal

2. Evaporation of cathode

3. Evaporation of $\mathrm{MoO}_{\mathrm{x}}(15 \mathrm{~nm})$ and ITO $(5 \mathrm{~nm})$

4. Sputtering of ITO $(60 \mathrm{~nm})$

5. Annealing at $\mathrm{N}_{2}$ ambient

6. Evaporation of anode

Figure : $5.10 \mathrm{n}-\mathrm{Si} / \mathrm{MoO}_{\times} / \mathrm{ITO}$ solar cell structures (a) without barrier layer and (b) with evaporated thin ITO $(5 \mathrm{~nm})$ as a barrier layer. The structures shown are not to scale, and the $\mathrm{MoO}_{x}$ and ITO layer thicknesses have been exaggerated. 
Figure $5.11(\mathrm{a})$ shows the illuminated $J-V$ curves of the solar cells fabricated with and without ITO barrier layer, and Table 5.3 summarizes the photovoltaic parameters of the cells. The $\mathrm{n}-\mathrm{Si} / \mathrm{MoO}_{x} / \mathrm{ITO}$ solar cell without the barrier layer has a $V_{o c}$ of $0.554 \mathrm{~V}$, which is much less than those of the $\mathrm{n}-\mathrm{Si} / \mathrm{MoO}_{\times} / \mathrm{PEDOT}: \mathrm{PSS}$ cells studied in the previous section, with $V_{o c}$ of 0.579 and $0.592 \mathrm{~V}$ for the cells annealed in $\mathrm{N}_{2}$ and UV-ozone respectively. In contrast, $\mathrm{n}-\mathrm{Si} / \mathrm{MoO}_{x} / \mathrm{ITO}$ solar cell with the barrier layer can achieve a $V_{o c}$ of $0.591 \mathrm{~V}$. The improvement in the $V_{o c}$ of the $\mathrm{n}$ $\mathrm{Si} / \mathrm{MoO}_{x} / \mathrm{ITO}$ solar cells cannot be explained in terms of $J_{s c}$ since the one without barrier layer has slightly higher $J_{s c}=34.3 \mathrm{~mA} / \mathrm{cm}^{2}$ as compared to the one with the barrier layer having $J_{s c}=32.1 \mathrm{~mA} / \mathrm{cm}^{2}$. From Fig. 5.11(b) which plots the dark J-V curves of the $\mathrm{n}-\mathrm{Si} / \mathrm{MoO}_{x} / \mathrm{ITO}$ solar cells fabricated with or without thin ITO barrier layer, it is observed that for applied voltage greater than $0.5 \mathrm{~V}$, the dark current of the cell with the barrier layer is lower, which is consistent with the improvement in $V_{o c}$ seen. 
(a)

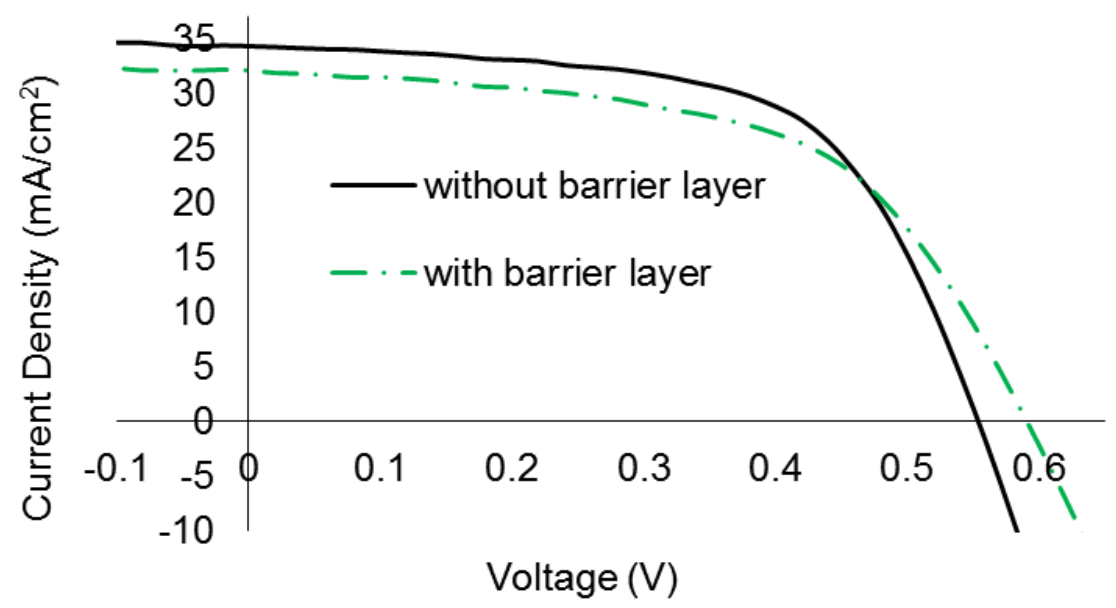

(b)

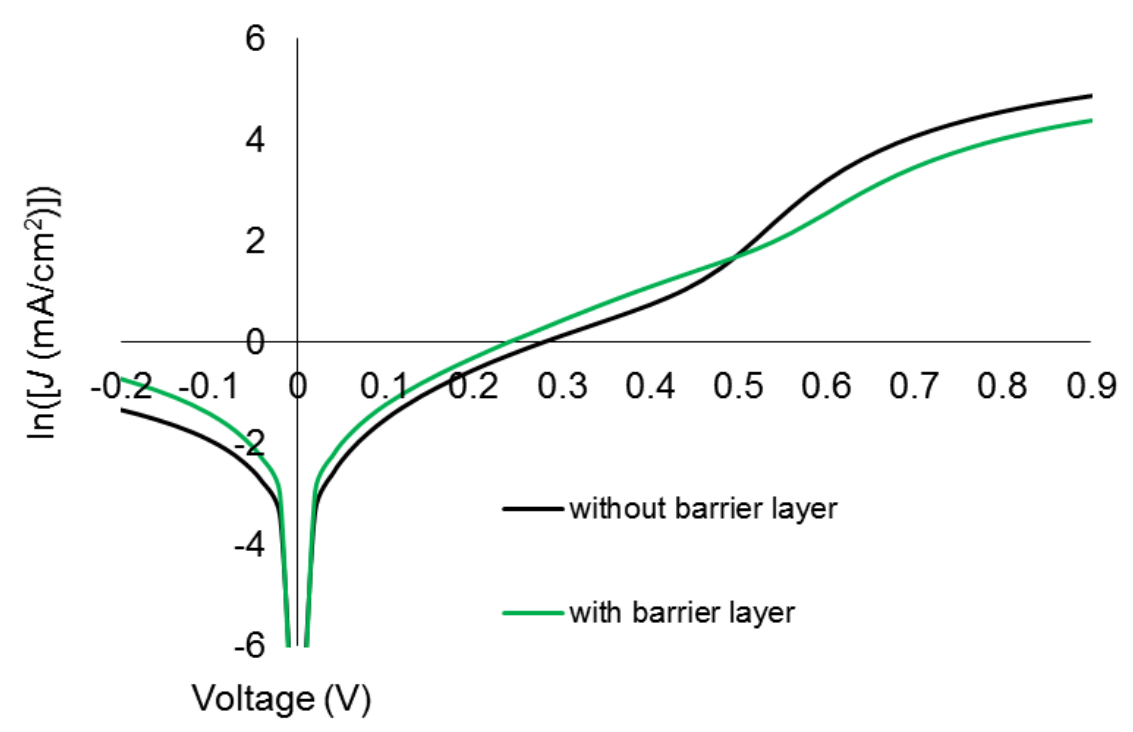

Figure : 5.11 (a) Illuminated J-V curves and (b) dark J-V curves of n-Si/MoOx/ITO solar cells fabricated with or without thin ITO barrier layer. 
Table 5.3 : Photovoltaic parameters for $n-S i / M{ }_{x} / I T O$ solar cell with or without thin ITO barrier layer.

\begin{tabular}{|l|c|c|}
\cline { 2 - 3 } \multicolumn{1}{c|}{} & $\begin{array}{c}\text { without } \\
\text { barrier layer }\end{array}$ & $\begin{array}{c}\text { with thin }(5 \mathrm{~nm}) \text { ITO } \\
\text { as a barrier layer }\end{array}$ \\
\hline$J_{s c}\left(\mathrm{~mA} / \mathrm{cm}^{2}\right)$ & 34.3 & 32.1 \\
\hline$V_{o c}(\mathrm{~V})$ & 0.554 & 0.591 \\
\hline FF $(\%)$ & 60.9 & 56.3 \\
\hline PCE (\%) & 11.6 & 10.7 \\
\hline
\end{tabular}

We know from the literature $[174,175]$ that exposure to water moisture in the air reduces the $\mathrm{MoO}_{x}$ work function, which is an important parameter that controls the energy level alignment and effectiveness of electron blocking on n-Si. From our previous experiments in section 5.3.2, we found that a $5 \mathrm{~nm}$ thin layer of ITO can protect $\mathrm{MoO}_{x}$ against water based PEDOT:PSS solution. The $\mathrm{n}-\mathrm{Si} / \mathrm{MoO} \times /$ TTO solar cell with a barrier layer is believed to have similarly protected the surface of the asdeposited $\mathrm{MoO}_{\mathrm{x}}$ and result in the higher $V_{o c}$ observed. The concept of preservation of as-deposited $\mathrm{MoO}_{x}$ with a barrier layer has been previously demonstrated [179], with the use of copper phthalocyanine and C60 as a barrier layer with a thickness down to two monolayers. In our case, the thin ITO is used as a barrier layer for convenience since it can be evaporated directly after $\mathrm{MoO}_{x}$ evaporation. 
Interestingly, we noted from Table 5.3 that the FF of the $\mathrm{n}-\mathrm{Si} / \mathrm{MoO}_{x} / \mathrm{ITO}$ solar cell without barrier layer is slightly higher at $60.9 \%$ as compared to the one with the barrier layer of $56.3 \%$, despite its lower $V_{o c}$. It can be observed from Fig. 5.11(b) that for applied voltage less than $0.5 \mathrm{~V}$, there is an increased shunt current component which is correlated with the lower FF. The increased shunt leakage is suspected to be due to penetration of evaporated ITO into pinholes in the $\mathrm{MoO}_{x}$ layer, which facilitates direct conduction between the $\mathrm{n}-\mathrm{Si}$ and the sputtered ITO, bypassing the $\mathrm{MoO}_{x}$. Further morphology characterization is needed to verify this assertion. In summary, although we found that the $\mathrm{n}-\mathrm{Si} / \mathrm{MoO}_{x} / \mathrm{ITO}$ solar cell with the thin ITO barrier layer can achieve a high $V_{o c}$ of $0.591 \mathrm{~V}$, however, its PCE is still lower at $10.7 \%$ as compared to the cell without the barrier layer of $11.6 \%$, owing to its lower $J_{s c}$ and FF.

\subsubsection{Influence of interfacial Si native oxide on Si/MoOx solar cell}

(a)

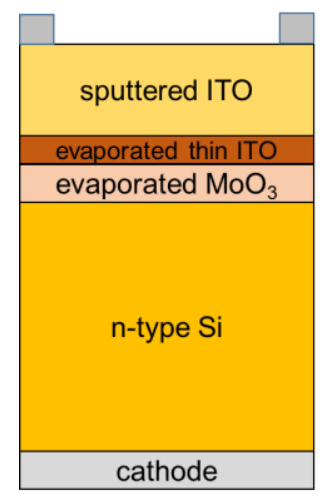

(b)

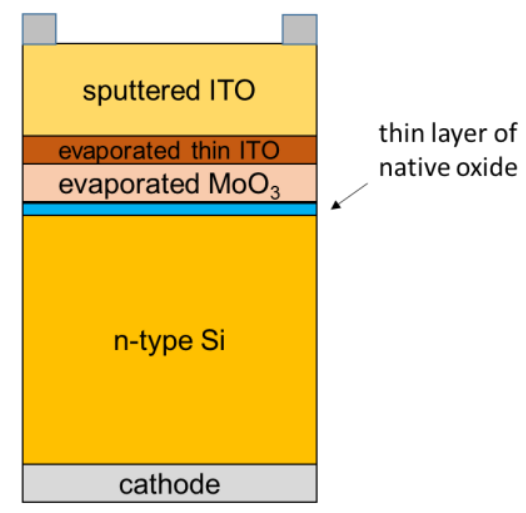

Figure 5.12 : n-Si/MoO $/$ ITO solar cell (a) without a native oxide and (b) with a thin layer of Si native oxide. 
A thin Si native oxide layer has been shown to play a critical role in enhancing the performance of Si/PEDOT:PSS hybrid heterojunction solar cell $[103,180]$. This is attributed to better surface passivation and enhanced surface dipole effect. In this section, we study the effect of having a thin Si native oxide on the performance of the $\mathrm{n}-\mathrm{Si} / \mathrm{MoO}_{x} / \mathrm{ITO}$ solar cell, whose structure is shown in Fig 5.12(b). It is believed that the native oxide may similarly improve the surface passivation and enhance the surface dipole to increase the Schottky barrier height developed between $\mathrm{Si}$ and $\mathrm{MoO}_{\mathrm{x}}$.

After the back contact deposition within the e-beam evaporation chamber, a reference solar cell is taken out and immediately prepared and put inside the evaporation chamber again for $\mathrm{MoO}_{\mathrm{x}}$ deposition. This process will result in a direct contact between the $\mathrm{n}-\mathrm{Si}$ and $\mathrm{MoO}_{x}$ layers as seen in Fig. 5.12(a). Whereas for the cell introduced with a native oxide, the Si surface was deliberately exposed to ambient air for $2 \mathrm{~h}$ after HF etching, before being put inside the evaporation chamber again for $\mathrm{MoO}_{x}$ deposition. This process will result in a thin native oxide interface layer with the thickness of about $1.8 \mathrm{~nm}$ [102] formed between $\mathrm{n}$-Si and $\mathrm{MoO}_{x}$ film. 
(a)

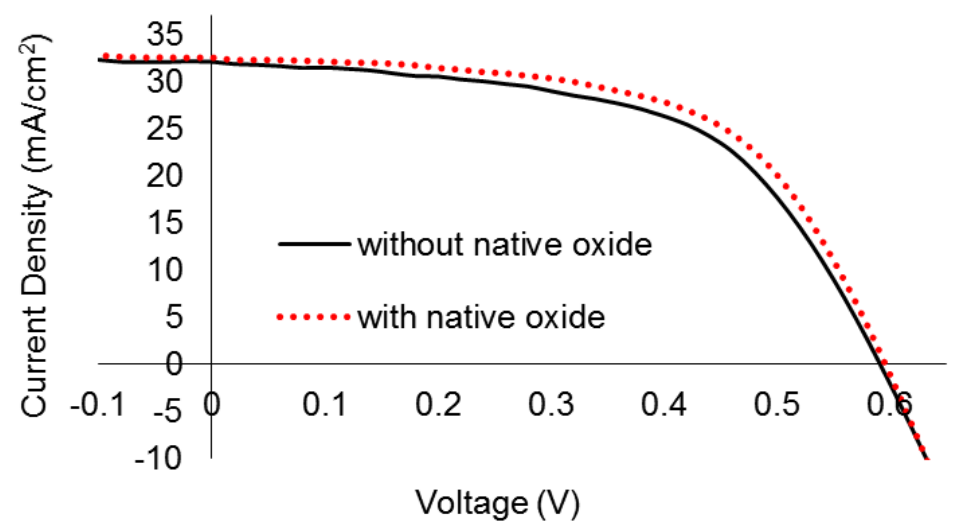

(b)

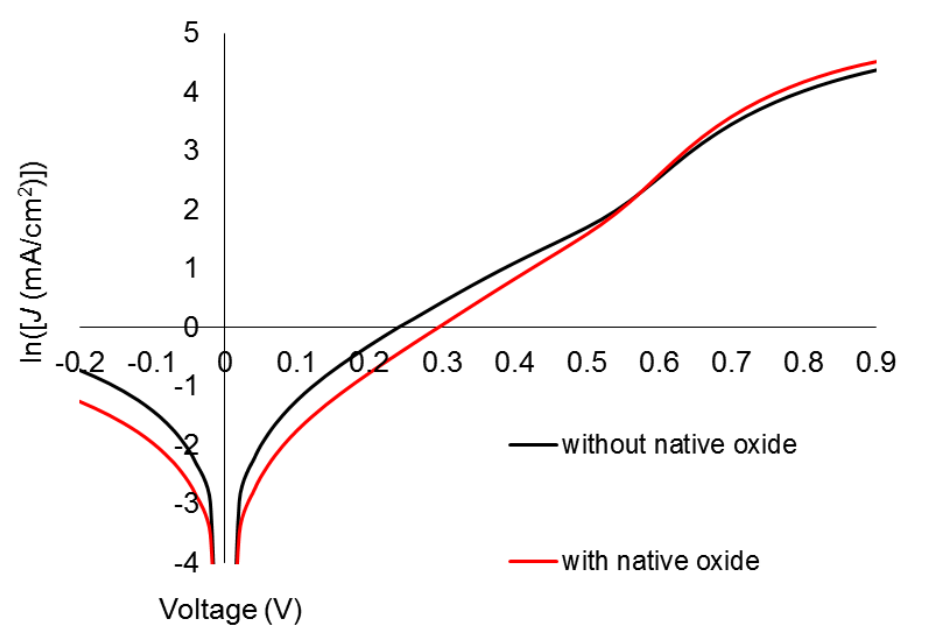

Figure 5.13 : (a) Illuminated and (b) dark $J$ - $V$ curves of $n-S_{i} / M O_{\rtimes} /$ ITO solar cell with and without native oxide at the $\mathrm{n}-\mathrm{Si} / \mathrm{MoO} \times$ interface.

Figure $5.13(a)$ shows the illuminated $J-V$ curves of $n-S i / M o O \rtimes / I T O$ solar cells with and without native oxide at the $\mathrm{n}-\mathrm{Si} / \mathrm{MoO}_{\mathrm{x}}$ interface, while their photovoltaic parameters are shown in Table 5.4. We found that the cell with native oxide has higher FF of $58.9 \%$ as compared to $56.3 \%$ for the cell without native oxide, as seen in Table 5.4. The increased FF is correlated to the reduced ohmic leakage current for bias voltage less than $0.52 \mathrm{~V}$, as seen in the dark $\mathrm{J}$ - $V$ curves shown in Fig. 
$5.13(b)$. The reduced ohmic leakage current is attributed to the presence of the thin native oxide. We found that the $V_{o c}$ is essentially similar for the solar cells with and without the native oxide layer, which is consistent with the relatively similar magnitude of dark current of the solar cells with and without the native oxide layer, around the bias voltage range of $0.55-0.65 \mathrm{~V}$. It is possible that the Schottky barrier height for the solar cell without the native oxide is already sufficiently high to block electrons, and hence the additional native oxide provides only marginal improvement in the blocking effect. The $J_{s c}$ of the solar cells is relatively similar at $32.1-32.5 \mathrm{~mA} / \mathrm{cm}^{2}$, which is not unexpected since the native oxide is relatively thin at $1-2 \mathrm{~nm}$ [102]. Overall, it is noted that arising mainly from the improvement in the $\mathrm{FF}$, the cell with a thin native oxide can reach $11.4 \%$ efficiency, which is much improved as compared to the cell without the native oxide layer of $10.7 \%$.

Table 5.4 : Photovoltaic parameters of $n-S i / M o O x / I T O$ solar cell with and without native oxide at the $\mathrm{n}-\mathrm{Si} / \mathrm{MoO}_{\mathrm{x}}$ interface.

\begin{tabular}{|l|c|c|c|c|}
\hline & $\begin{array}{c}\mathrm{J}_{\mathrm{sc}} \\
\left(\mathrm{mA} / \mathrm{cm}^{2}\right)\end{array}$ & $\begin{array}{c}\mathrm{V}_{\mathrm{oc}} \\
(\mathrm{V})\end{array}$ & $\begin{array}{c}\mathrm{FF} \\
(\%)\end{array}$ & $\begin{array}{c}\mathrm{PCE} \\
(\%)\end{array}$ \\
\hline without native oxide & 32.1 & 0.591 & 56.3 & 10.7 \\
\hline with native oxide & 32.5 & 0.595 & 58.9 & 11.4 \\
\hline
\end{tabular}




\subsection{Conclusion}

In conclusion, we have fabricated and optimized Si/transition metal oxide (Si-TMO) heterojunction solar cell based on planar $\mathrm{n}$-Si substrate and molybdenum oxide $\left(\mathrm{MoO}_{\mathrm{x}}\right)$. We studied the influence of $\mathrm{Si}$ substrate doping concentration $\left(N_{D}\right)$ on $\mathrm{n}$ $\mathrm{Si} / \mathrm{MoO}_{\mathrm{x}}$ solar cell and found that over the range $N_{D}=10^{14}-10^{16} / \mathrm{cm}^{3}$, the $V_{\text {oc }}$ increases with $N_{D}$ from $0.518 \mathrm{~V}$ to $0.554 \mathrm{~V}$. The $V_{\text {oc }}$ decreases to $0.503 \mathrm{~V}$ at $N_{D}=$ $10^{17} / \mathrm{cm}^{3}$, which is related to the increased ohmic shunt leakage current component. On the other hand, the $J_{s c}$ decreases with increasing $N_{D}$ due to the reduced diffusion length in $\mathrm{Si}$. The FF also decreases with $N_{D}$, which is related to the increased ohmic shunt current leakage that is observed under low forward bias in the dark $J-V$ curve. These mechanisms render the efficiency almost independent of doping at 10.1 to $10.2 \%$ for $N_{D}=10^{14}-10^{16} / \mathrm{cm}^{3}$, which then drops to $6.9 \%$ at higher $N_{D}=10^{17} / \mathrm{cm}^{3}$. It is found that using an evaporated $5 \mathrm{~nm}$ thin ITO layer on top of $\mathrm{MoO}_{x}$ as a water barrier, $\mathrm{n}-\mathrm{Si} / \mathrm{MoO}_{x}$ solar cell with PEDOT:PSS electrode can achieve $V_{o c}=0.592 \mathrm{~V}, \mathrm{FF}=67.3 \%$ and $\mathrm{PCE}=11.3 \%$. Subsequently, we studied $\mathrm{n}-\mathrm{Si} / \mathrm{MoO}_{\mathrm{x}}$ solar cell with sputtered ITO electrode with or without thin evaporated ITO as a barrier layer. We found that the highest PCE $=11.6 \%$ and $J_{\mathrm{sc}}$ $=34.3 \mathrm{~mA} / \mathrm{cm}^{2}$ are observed for the solar cell without the barrier layer. Although the solar cell with the barrier layer yields $V_{o c}=0.591 \mathrm{~V}$, which is significantly higher than $V_{\text {oc }}=0.554 \mathrm{~V}$ for those without the barrier, however, it has lower PCE $=10.7 \%$ due to its lower $J_{s c}$ and FF. A further introduction of native oxide at the $n-S i / M_{0} O_{x}$ interface can improve the FF of the device with the barrier layer, and enhance the efficiency to $11.4 \%$. 


\section{Chapter 6. Simulation of PV-Tower solar cell for high power over small footprint application}

In this chapter, we propose and present the concept of a novel solar cell design that can deliver high power over small footprint. It is named as PV-Tower solar cell, and it is ideal for solar energy harnessing for powering sensors and circuit in the internet of things (IOT) application. In our study, a full simulation of the operation and characteristics of the PV-Tower solar cell will be carried out to demonstrate its feasibility. To begin with, the motivation behind developing a solar cell for high power over small footprint application will be explained in relation to proliferation of energy autonomous Internet-of-Things Smart Node (IoT-SN). Subsequently, we will explain how PV-Tower solar cell satisfies the requirement for this application and how it can be produced using industrially viable processes. Finally, we will present and analyze the simulation results obtained for the device to demonstrate its excellent performance in terms of power delivery per unit foot print area.

\subsection{Introduction and motivation}

Currently, there is a growing interest on Internet of Things (loT) where small and intelligent wireless sensors are deployed throughout the environment, forming a network for the collection and exchange of information. This enables large-scale real-time monitoring in areas such as smart building, traffic management, environmental monitoring, personal health care, etc [56, 114-119]. It is estimated 
that in the near future, billions of physical objects will be connected using wireless IoT Smart Nodes (IoT-SN), allowing enhanced data collection and interactive control of the physical world around us [117]. IoT smart node (IOT-SN) is a selfcontained device which can collect data from its surrounding and send the information remotely to a data collecting unit. Typically several loT-SNs will be employed to form data collection networks that are connected to the internet. A key challenge in loT-SN is the provision of energy for sensing, electronics, and communication. It is imperative that loT-SN are energy autonomous, that is, able to harvest environmental energy [55-58] and store in supercapacitors/batteries to power its operation $[181,182]$. This will eliminate the need for battery replacement and allow loT-SN to be installed in "deploy and forget" mode [57]. Among the environmental energies available, harvesting of light energy using photovoltaic (PV) is the most promising [120], as it is one of the most efficient approaches and light energy is widely available in both outdoor and indoor environments [59, 60, $120,121]$.

Typical IoT-SN has footprint of tens of square centimeters $[56,61,116,120,122$, $181,183,184]$. It is desirable to reduce the size as much as possible, down to several square centimeters or even square millimeters range [55, 59-62], to realize less intrusive sensors with lower power consumption. For example, a state-of-theart loT-SN has a sensor node with dimensions of $0.5 \times 1.5 \times 2 \mathrm{~mm}^{3}$, designed to be safely inserted into human eyes to monitor intraocular pressure on glaucoma patient [185]. It incorporates a $0.07 \mathrm{~mm}^{2}$ solar cell to harvest light energy entering 
the eyes, even under low light intensity condition. The trend towards miniaturization of loT-SN will lead to less area for solar cells and consequently insufficient energy harvested to power the loT-SN. Indeed, IOT-SN often needs solar cell with a footprint comparable or larger than the rest of the circuit $[56,59,122]$.

The energy constraint can be alleviated to a certain extent by reducing and managing the energy requirement of loT-SN [63-66], such as lowering the regularity of sensing and communication. This, however, compromises the availability of timely information. It can also be addressed by having more compact integration of PV to IoT-SN. A common approach is to use multiple layer PCB or substrate system, where most of the electronics are placed on the lower layer, while the solar cell on the top most layer [59]. This maximizes the area available for the solar cell, but increases the volumetric footprint, and may hinder the operation of sensors underneath the solar cell. Another approach is to use solar cells based on materials such as III-V semiconductors [60], or a-Si [58, 67], instead of conventional crystalline Si. These materials have energy bandgaps suitable for indoor visible light and offer better absorption than $\mathrm{Si}$, and hence can more efficiently absorb commonly used artificial indoor light sources, e.g. fluorescence or LED lamp. However, III-V semiconductors are costly, while a-Si is inefficient in capturing outdoor sunlight compared to $\mathrm{Si}$. All the above issues constraining PV energy harvesting in IoT-SN can be resolved if there is a way to drastically improve the output power per unit footprint of the solar cell. Indeed, the availability of powerful 
and small footprint solar cell that can fit into $\mathrm{mm}$ [55] or $\mathrm{cm}$ [57] scale loT-SN circuit will be critical in enabling the mass deployment of energy autonomous loT-SN.

Conventional Si solar cell has its metallurgical junction (the plane between $\mathrm{n}$-doped and p-doped regions of the solar cell) aligned parallel to its base plane, as seen in Fig. 6.1(a). As incident light from the top is mostly absorbed just over tens of micrometers thickness [186], the height of the solar cell is usually kept to a minimum to lower the material cost. In practice, Si substrate thickness of the order of $\sim 100$ to $200 \mu \mathrm{m}$ is adopted for robust mechanical handling in the PV industry, and the trend is towards using even thinner Si wafers for cost saving. Given the typically much larger surface dimensions compared to its height, therefore conventional solar cell is essentially a two-dimensional planar device. The surface available for light absorption is defined by the top surface area, which is also its footprint of dimensions $a \times b$ (see Fig. 6.1(a)). To increase the output power will inevitably require an increase in its footprint, which is undesirable for the loT-SN application. 


\subsection{PV-Tower cell concept}

\subsubsection{Merit of PV-Tower compared to conventional solar cell}

(b) electrodes
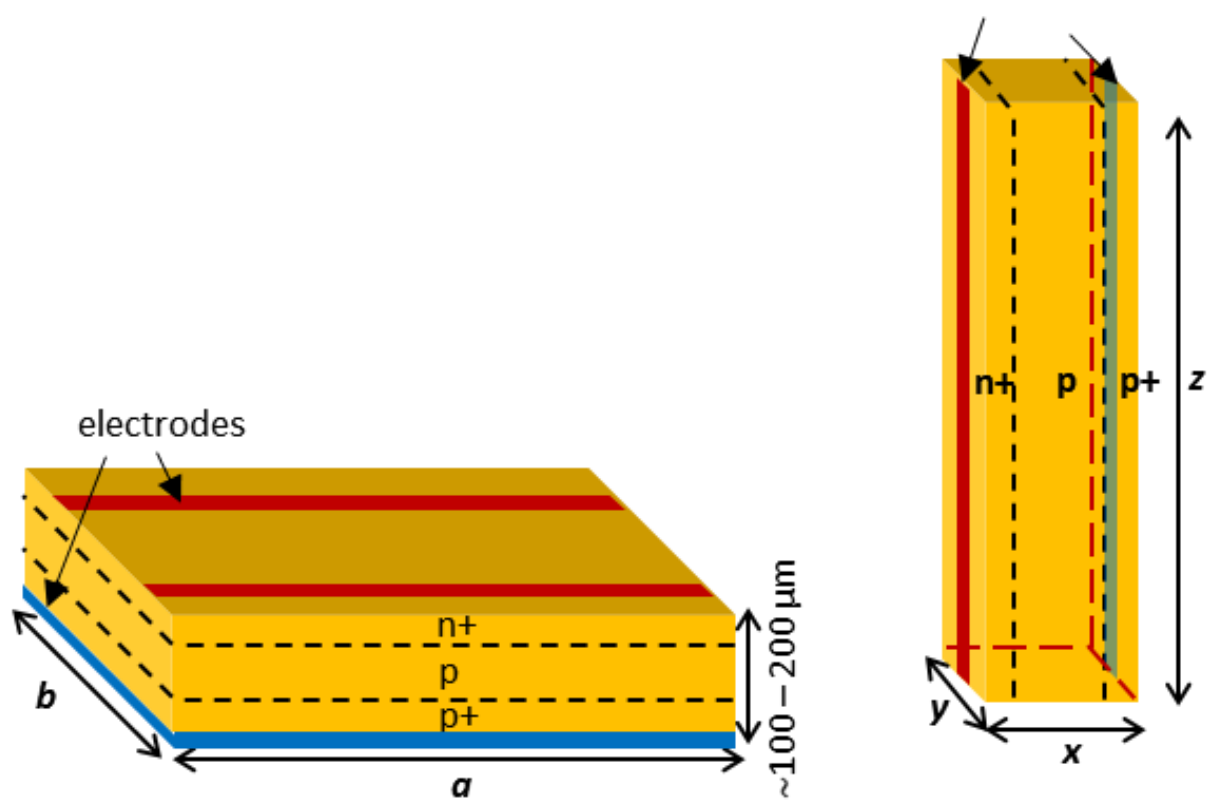

Figure 6.1 : Schematic of (a) conventional planar solar cell and (b) PV-Tower cell.

In this project, we propose a novel PV-Tower solar cell that offers a huge surface area for light absorption, and yet occupies only a small footprint. As shown in Fig. 6.1(b), the PV-Tower has a tower structure with the metallurgical junction aligned perpendicular to the base plane. It has a simple $n+-p-p+($ or $n+-n-p+)$ front-back structure with metallization on two opposite sidewalls. Its footprint is $x \times y$, where the length $x$ is of the order of diffusion length $(\sim 100-200 \mu \mathrm{m})$, to ensure that the carriers can be separated to generate photocurrent before recombination. The novelty of PV-Tower lies in the fact that its height $z$ can be significantly increased 
to boost the surface area available for light absorption without enlarging its footprint, in contrast to the conventional planar solar cell. Indeed the height $z$ can increase to that of loT-SN circuit packaging typically in $\mathrm{mm}$ or $\mathrm{cm}$ scale, to drastically boost optical absorption and power generation.

Therefore, the PV-Tower is a three-dimensional device with a high aspect ratio, and it can harvest light energy not only from the top surface but more importantly from the four sidewalls that offer huge surface area. As an illustration, assuming the same footprint for both conventional and PV-Tower solar cells $(a \times b=x \times y)$ and $x=100 \mu \mathrm{m}$, the surface area available for light absorption for PV-Tower is > 50 times that of conventional planar cell if $z=2.5 \mathrm{~mm}$, and $>500$ times if $z=2.5$ $\mathrm{cm}$. This signifies a huge improvement in the power that can be generated over the same footprint.

Conversely, for the same output power, the footprint of the PV-Tower can be several hundred times smaller as compared to a planar solar cell. This thus allows a very compact integration with miniaturized ultra-small IoT-SN. It should be highlighted that the excellent mechanical properties of crystalline Si [187-189], with Young's modulus of $\sim 130 \mathrm{GPa}$, allows such Si towers with high aspect ratio (>100) to be realized without self-buckling [190]. They are therefore practical and achievable. 
Our proposed PV-Tower solar cell offers a number of advantages as compared to conventional planar solar cells for energy harvesting in loT-SN:

1. It drastically increases the amount of light energy that can be harvested over a given footprint and thus can serve as a compact, efficient and high power source. For the same output power, it will occupy a drastically reduced footprint as compared to planar cells and is thus ideal for loT-SN application.

2. As it has multiple surfaces facing different directions, it can effectively harvest diffuse light that is dominant in indoor environments where many IoT-SN will be deployed [59]. This is in contrast to planar cells that are designed to capture directional light, which is thus not as efficient under indoor environments.

3. It has a large surface area to volume ratio, implying a large output power with minimal semiconductor material usage, leading to the more costeffective device.

4. As each PV-Tower is modular, they can be readily configured and customized as an array or distributed across the IoT-SN to meet its power requirement. For example, several PV towers can be connected in series to deliver a specific output voltage, or in parallel to boost the overall power supply. Such PV Tower power supply module can be integrated with loT-SN in a "plug-and-play" manner with great flexibility.

5. Due to its modularity, it is also possible to integrate PV Towers fabricated using different semiconductor materials to offer the best absorption characteristics for a given lighting environment. 
6. It can be encapsulated by low cost transparent plastic lenses (e.g. rectangular lenses) to focus light onto the PV-Tower and further enhance absorption over a small footprint. Such encapsulation also serves to strengthen the mechanical robustness of the PV-Tower solar cell.

Therefore the proposed PV-Tower solar cell is compact and efficient, and can effectively harvest diffuse light to power energy autonomous loT-SN in loT network. Very importantly, as will be illustrated, it can be fabricated on a large scale and at low cost, using processes compatible with existing Si PV technology.

Previously, Verlinden et al. [123] developed the Sliver ${ }^{\circledR}$ cell concept where the solar cell is cut into strips, positioned horizontally and connected in a huge array. This allows light to be intercepted from the top and bottom surfaces, doubling the absorption area over the same footprint. A 3D solar cell concept with a large number of tower structures packed in high density over a large area has also been proposed by Ready et al. [124, 125]. The idea is to use the closely spaced towers for light trapping to improve the overall optical absorption as seen in Fig 6.2(a). The height of the individual tower proposed is $100 \mu \mathrm{m}$, which is slightly larger than the base dimensions of $40 \mu \mathrm{m} \times 40 \mu \mathrm{m}$ and this resulted in an increase in absorption by three times [124] despite increased geometric surface area of 11 . This is due to the fact that the increased geometric surface area increased the chance to capture secondary light reflected from other towers instead of primary light from the environment. This in contrast to the PV-Tower concept which aims to increase the 
surface area to collect primary light, which is directly proportional to its height as seen in Fig. 6.2(b).

(a)

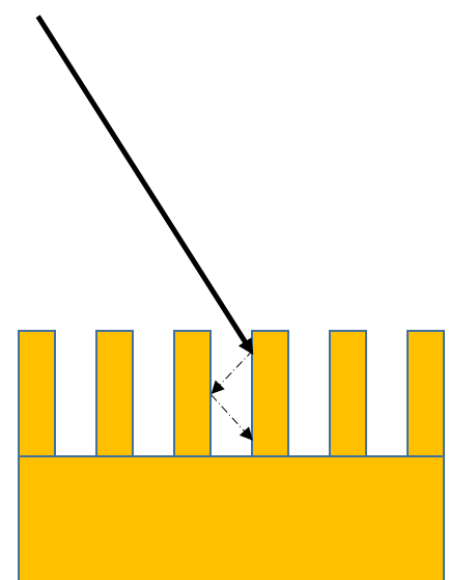

(b)

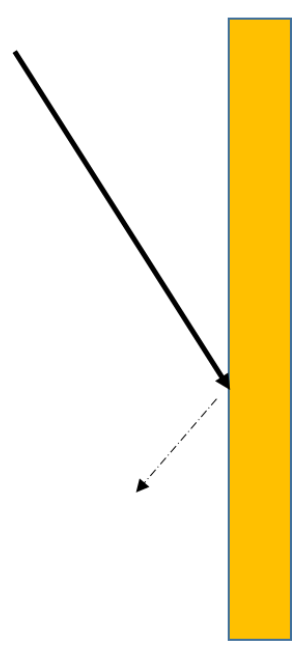

$\searrow$ Primary light from environment

Secondary light

Figure 6.2 : Distinction between (a) light trapping concept based on arrays structure and (b) direct increase of active surface area by PV-Tower.

Bernardi et al. [128] demonstrated a stacking of conventional PV modules in a three-dimensional structure to increase the surface area for absorption over a given footprint. However, the use of conventional PV modules in this arrangement wastes a significant amount of costly semiconductor material [128] and is too complicated to replicate in a smaller scale appropriate for miniaturized loT-SN. The above concepts reported are different from our proposed PV-Tower cell in several aspects: (i) Our PV-Tower cell is positioned vertically and has heights up to centimetre range, resulting in an increase in absorption area by several hundred times compared to a planar cell with the same footprint. Note that such centimetre 
scale height solar cell with very high aspect ratio has not been attempted before; (ii) our PV-Tower cell is designed to operate individually or in a small number in series/parallel connection, which is in contrast to the above reports that are mostly based on an array of a huge number of closely spaced structures that suffer from shadowing effect; (iii) our PV-Tower is compact, efficient, and occupies only a small footprint that is ideal for powering loT-SN, in contrast to the other works that are designed for large area based solar cells and (iv) the design of PV-Tower as an individual solar cell allows large active surface area per given semiconductor volume, resulting in an efficient use of the material.

\subsubsection{Industrially Viable Fabrication Process for PV-Tower}

(a)

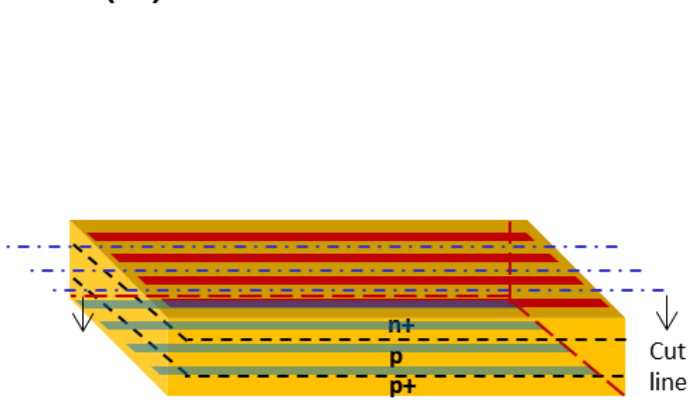

(b)

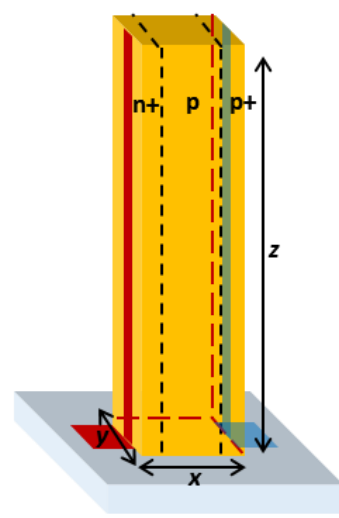

(c)

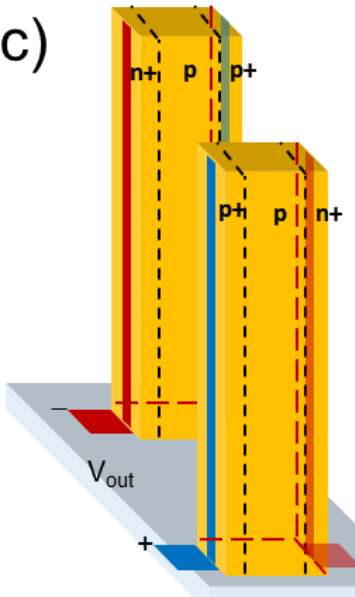

Figure 6.3 : (a) conventional Si wafer solar cell with patterned electrodes on both surfaces and cut along the blue dotted lines to form PV-Towers, (b) Individual vertically aligned PV-Tower solar cell and (c) Multiple PV-Tower solar cells connected in series on a rigid insulating substrate (not to scale). 
C-Si based PV-Tower with a rectangular cross-section, and a front-back $n+-p-p+$ (or $n+-n-p+)$ junction structure can be fabricated using processes compatible with existing Si PV technology, as described in Fig. 6.3(a). The proposed process begins with the fabrication of conventional $n+-p-p+$ junction solar cell on a large area double-side polished Si wafer using conventional diffusion process. An antireflective coating (ARC) and parallel metal grids can be deposited on both sides of the wafer. The wafer can be pasted on a flexible dicing sheet and coated with a temporary protective layer on top. It will be cut along a direction parallel to and in between the metal grids using either diamond cutter, laser ablation or selective wet chemical etching to form separate PV-Tower cells, which are held together after the cutting by the dicing sheet. The sidewall surfaces of the PV-Towers can be passivated by dry or wet chemical processes to minimize surface recombination. Apart from ARC, we can study fabricating Si nanostructures on the sidewalls to minimize reflection. Finally, the dicing sheet and the top protective layer can be removed to obtain individual PV-Towers. For the electrodes on the two sides, besides metal grids, the use of transparent conductors such as ITO, FTO can be considered. Based on the above proposed process, Si Towers with cross-sectional dimensions of $x \sim 100-200 \mu \mathrm{m}, y$ ranging from several hundred micrometers to millimeters, and $z$ reaching up to several centimeters can be fabricated. Individual PV-Tower cell can be mounted vertically and positioned on an insulating substrate using prefabricated grooves or epoxy glue for PV testing, as shown in Fig. 6.3(b). 
The majority of the processes involved in the fabrication of PV-Towers are compatible with those used in the industry fabrication of Si wafer solar cells. There are challenges faced that include achieving well-passivated high quality sidewalls and ensuring the mechanical robustness of the high aspect ratio PV-Tower and its secured vertical positioning on an insulating substrate. Apart from the Si PV-Tower with a rectangular cross-section and front-back junction structure, other PV materials (e.g. a-Si) with different cross-section shapes, doping profiles and fabrication methods (e.g. PECVD) can be explored to leverage on the advantages offered by the different absorption characteristics and fabrication processes.

Multiple PV-Tower cells will be connected in series and/or parallel by metal strips running on the substrate, as shown in Fig. 6.3(c), to form compact modular PV Tower power supply that can be configured to achieve specific voltage or power requirements. Two output terminals will be formed to facilitate connection to loTSN. Note that it is not desirable to have too many PV Towers in the power supply due to the concern of shadowing that will lower the overall power output. Just several PV Towers are generally needed to meet the output voltage requirement, while the current requirements can be met by having wider (i.e. larger $y$ ) or taller PV Towers. We will study protecting the entire power supply by transparent plastic encapsulation that also serves to focus light onto the PV Tower solar cells and enhance light absorption and power generation. 


\subsection{PV-Tower cell performance}

We will present PV-Tower cell performance based on numerical device simulation. Firstly, the active surface area per footprint, offered by PV-Tower cell concept will be illustrated. We will then perform numerical device simulation for the Si-based PV-Tower cell to illustrate its response to illumination, in particular at different incident angular directions. The effect of PV-Tower height to resistance losses will also be estimated based on the grid geometry.

\subsubsection{Active surface area per footprint}

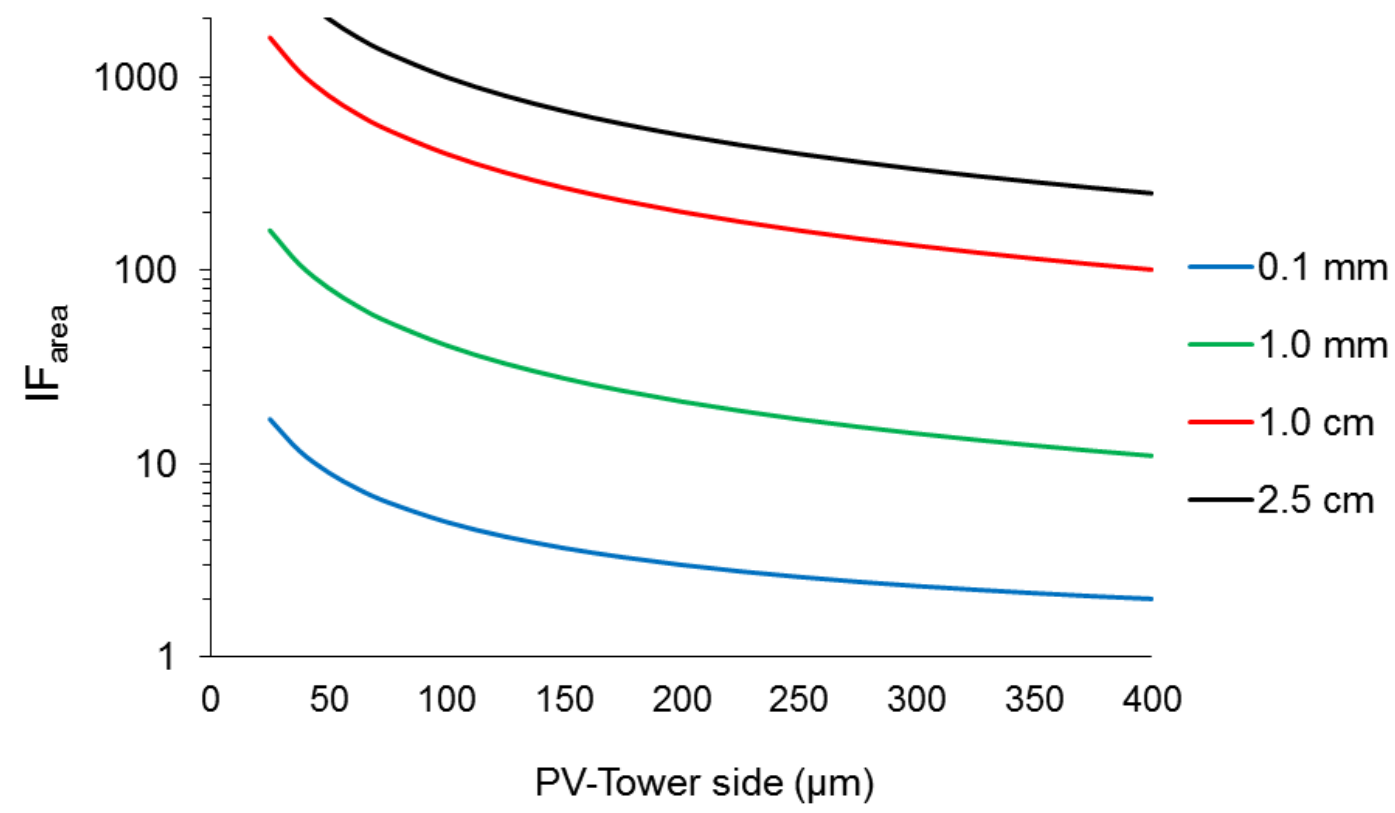

Figure 6.4 : Area improvement factor (IF area) for PV-Tower solar cells with a square cross-section and with various tower heights. 
One important figure of merit for PV-Tower is the area improvement factor (IFarea) which is defined as the ratio of the active surface area compared to its footprint. Referring to Fig. 6.1(b), the active surface comprises the four sides of the tower with an area of $2 \times(x \times z+y \times z)$, plus its top surface with an area of $(x \times y)$, whereas the footprint is just equal to the top surface area. Figure 6.4 shows the $\mathrm{IF}_{\text {area }}$ as a function of a PV-Tower having a square cross section $(x=y)$ and with various tower heights $z$. The square cross section is chosen for simplicity, although rectangular cross section PV-Tower can also be used. We can see in general that PV-Tower with smaller cross section will have better $\mathrm{IF}_{\text {area }}$ as compared to those with the larger cross section since the side wall area will contribute substantially to the total area when the footprint area is small. It is also observed that IF area increases with the tower height $z$, which is expected as the side wall area will be larger for a higher tower. As an illustration, considering PV-Towers with a square cross section of side $x=y=200 \mu \mathrm{m}$, it can yield IF area of $\approx 20$ and 500 for height $z$ of $1 \mathrm{~mm}$ and $2.5 \mathrm{~cm}$ height respectively. An even higher IFarea of 1000 can be achieved for the PV-Tower with $2.5 \mathrm{~cm}$ height by decreasing the side $x$ and $y$ to $100 \mu \mathrm{m}$. Therefore, we see from the results that the PV-Tower solar cell allows the active area for absorption to be significantly increased relative to the footprint by up to two or three orders of magnitude. It should also be noted, however, that the area improvement factor IFarea may not necessarily equate to power improvement factor $\left(\mathrm{IF}_{\text {power }}\right)$, defined as the ratio of the power generated by the PV-Tower relative to that generated by a solar cell with an active area that is equal to its footprint. It is expected that IF power will approach IF area under diffuse lighting condition when the four sides of the tower 
are illuminated. Another important aspect to consider is the series resistance losses from the finger grid, which stretches along the height of the tower as seen in Fig. 6.1(a), and increases with the tower height. This will limit the $\mathrm{IF}_{\text {power }}$ that could be achieved for the taller PV-Tower solar cell.

An important figure of merit for the design of PV-Tower solar cell is the ratio between the $\mathrm{IF}_{\text {power }}$ and $\mathrm{IF}_{\text {area, }}$ and hence it is important to study the effects that may lead to a discrepancy between them. In the following sections, we study the discrepancy which is caused by carrier collection response to light from different directions by using the 2D numerical device simulation software, Silvaco ATLAS ${ }^{\mathrm{TM}}$. Subsequently, we will present the difference in $\mathrm{IF}_{\text {power }}$ and $\mathrm{IF}_{\text {area }}$ due to the increased series resistance losses with height scaling using analytical equation.

\subsubsection{Si-based PV-Tower cell response with light incident from different directions}

In order to study the influence of carrier collection with light incident from various directions, we simulate its response as a function of the incident angle. Since the PV-Tower has a height that is far larger than its cross sectional dimension, and as the side wall constitutes a significant fraction of the total area, we study its response using the cylindrical coordinate with the axis centered at the axis of the PV-Tower, as shown in Fig. 6.5. The PV-Tower is mounted on a rotating axis, which is 
subjected to directional light. This will allow us to investigate the PV response of the PV-Tower solar cell with light incident from different directions onto the sidewall.

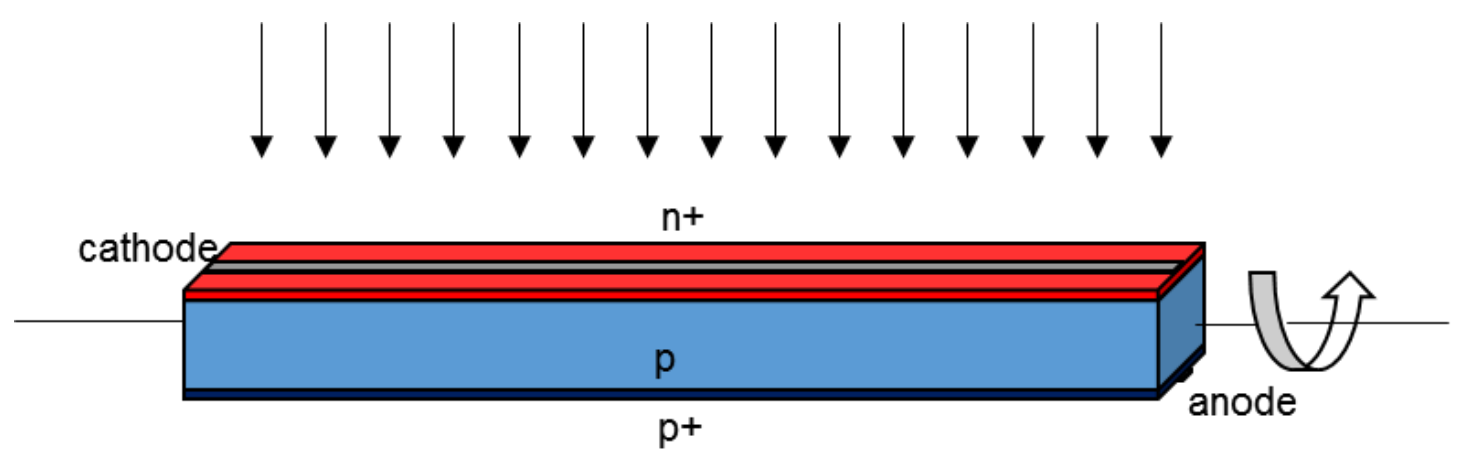

Figure 6.5 : Study of PV-Tower cell response to light with different incident directions.

The top view of the setup is also shown in Fig. 6.6, where the incident angle $\theta$ is defined. The PV-Tower has an $\mathrm{n}^{+}-\mathrm{p}-\mathrm{p}^{+}$structure, and the $\mathrm{p}$-type wafer is designed with a doping concentration $N_{A}=10^{16} / \mathrm{cm}^{3}$ since this is more commonly used in the conventional solar cell industry. The conventional solar cell is simulated in order to design for the practical industrial application. We set a bulk lifetime of $300 \mu$ s which is considered realistic to model typical c-Si wafer used in a commercial setting. The $200 \mu \mathrm{m} \times 200 \mu \mathrm{m}$ cross section is chosen since solar cell can typically be processed with a wafer thickness of $200 \mu \mathrm{m}$ and yet with acceptable mechanical robustness. The $\mathrm{n}^{+} \mathrm{Si}$ emitter is set to the thickness of $500 \mathrm{~nm}$ and doping concentration of $N_{D}$ $=10^{19} / \mathrm{cm}^{3}$, while the $\mathrm{p}^{+}$Si surface field is chosen with the same thickness of 500 $\mathrm{nm}$ and doping concentration $N_{A}=10^{19} / \mathrm{cm}^{3}$. For simplicity, the doping profiles are set to be uniform in all the layers. The $2 \mathrm{D}$ simulation was carried out using Silvaco 
ATLAS $^{\mathrm{TM}}$ software for a PV-Tower with a height of $1 \mu \mathrm{m}$ along the direction of PVTower axis. The response of the tower structure is proportional to its height. Therefore, an arbitrary height of $1 \mu \mathrm{m}$ was used to simulate the structure. For tower cell with different heights, the response can be scaled accordingly.

\section{normal to emitter}

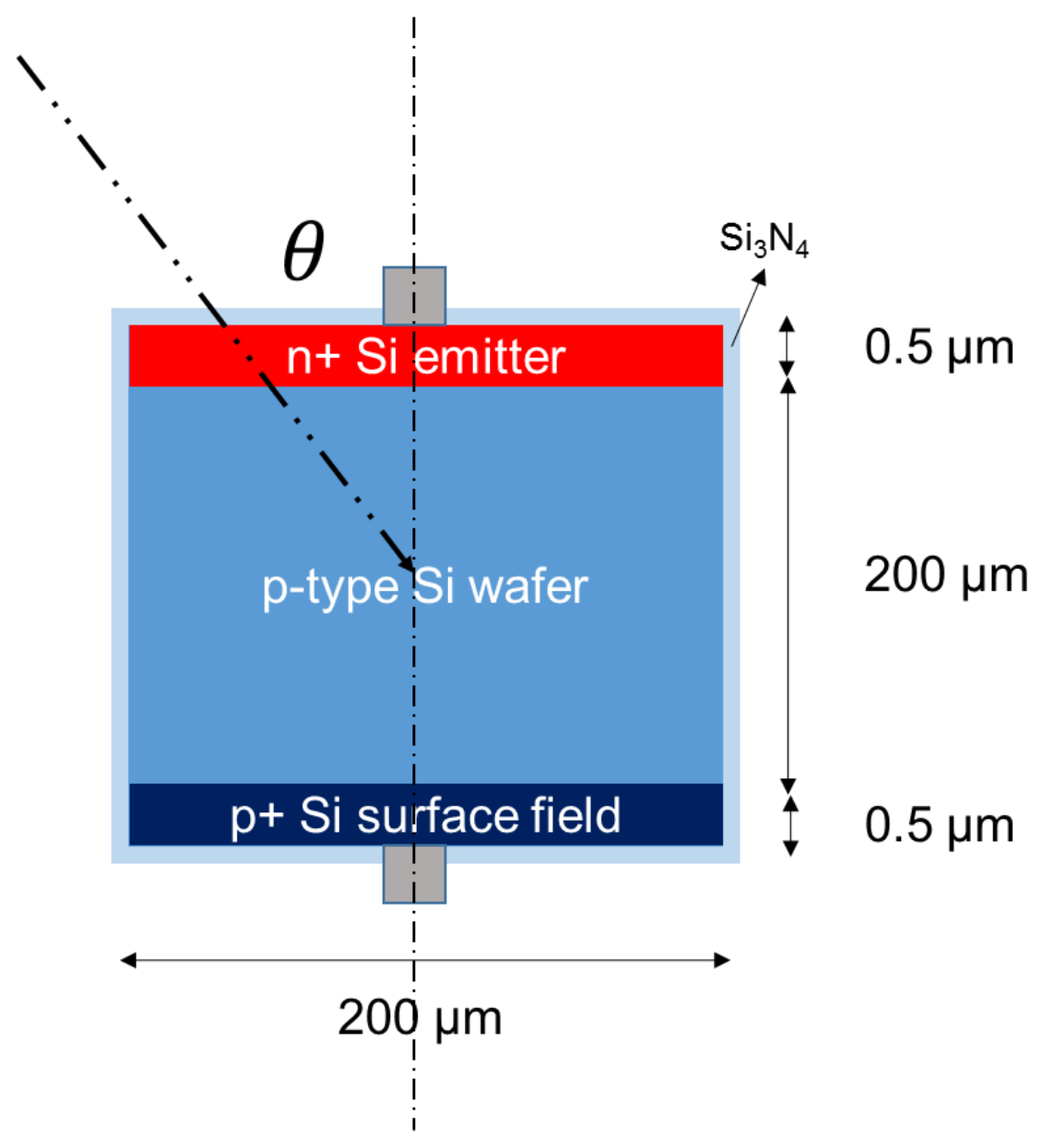

Figure 6.6 : Top view of PV-Tower with $200 \mu \mathrm{m} \times 200 \mu \mathrm{m}$ cross section and definition of the angular direction. 


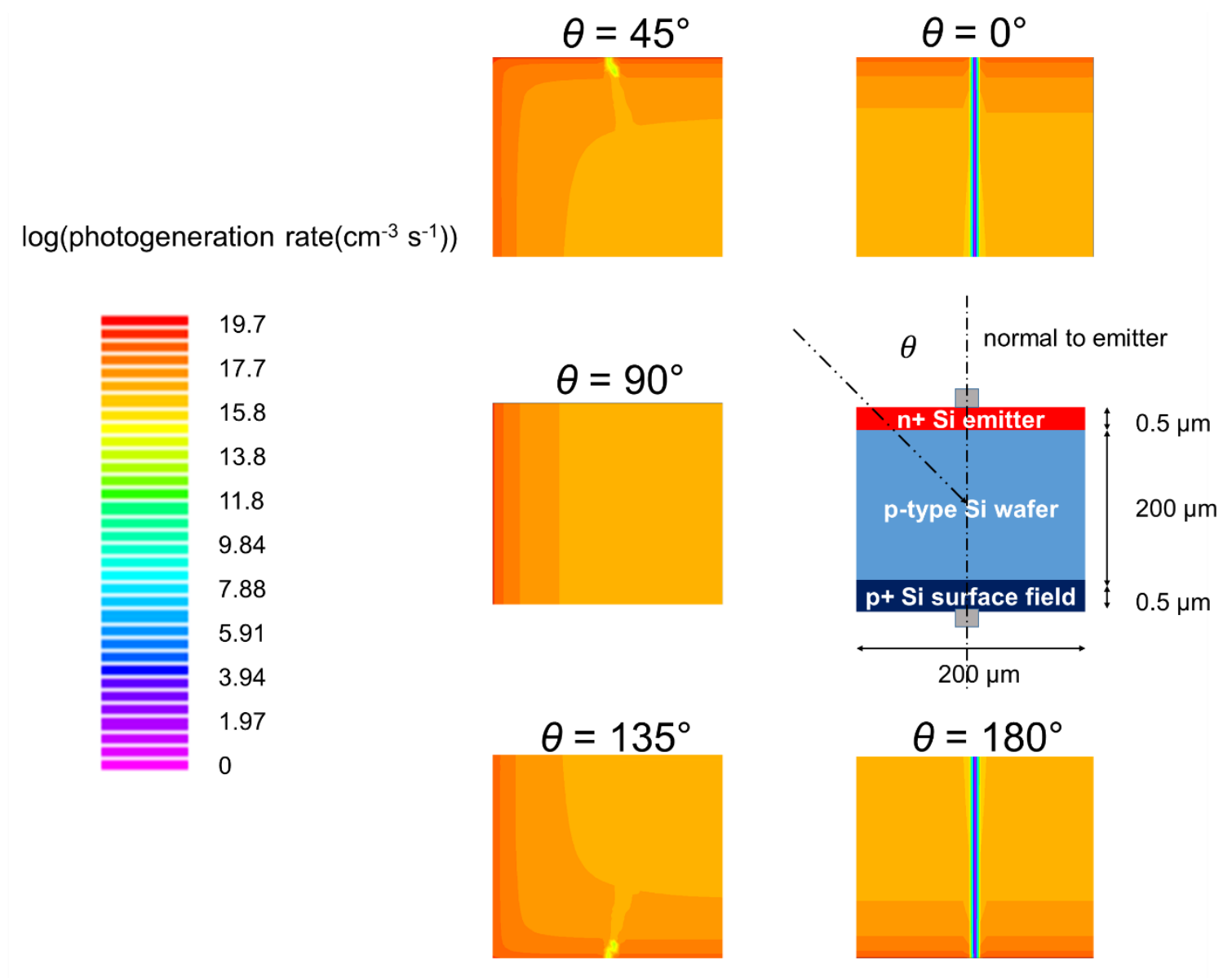

Figure 6.7 : Photogeneration profiles for the light with different incident angles.

Figure 6.7 shows the photogeneration profiles for the light incident from different directions. At $\theta=0$ and $180^{\circ}$, we observe intense photogeneration close to the emitter and surface field layer, respectively. Notice that the distinct lines seen in the results are due to grid shadowing. For the incident light with $\theta=90^{\circ}$, the intense photogeneration region occurs at the sidewall of the PV-Tower, without any grid shadow appearance. For the light incident diagonally with $\theta=45^{\circ}$ and $135^{\circ}$, it is observed that there is high photogeneration occurring at two sidewalls concurrently. Figure 6.8 shows the power generated for a PV-Tower solar cell with 
a height of $2.5 \mathrm{~cm}$ under AM1.5 $100 \mathrm{~mW} / \mathrm{cm}^{2}$ solar radiation. At $\theta=0^{\circ}$, the power output is $0.86 \mathrm{~mW}$, while at $\theta=180^{\circ}$ the power generated is slightly smaller at 0.83 $\mathrm{mW}$. The smaller power for $\theta=180^{\circ}$ is due to an increase in the distance required for carrier collection at the junction. At $\theta=90^{\circ}$, the power is at $0.91 \mathrm{~mW}$, which is higher than those at $\theta=0^{\circ}$ due to reduced grid shadowing. The power output peaks at $45^{\circ}$ and $135^{\circ}$ since the light illumination falls in the diagonal direction of the tower cross section, and absorption takes place strongly across two sidewalls, as also illustrated in the photogeneration profiles shown in Fig. 6.7. The enhanced response is also assisted by increased internal reflection under diagonal incident direction.

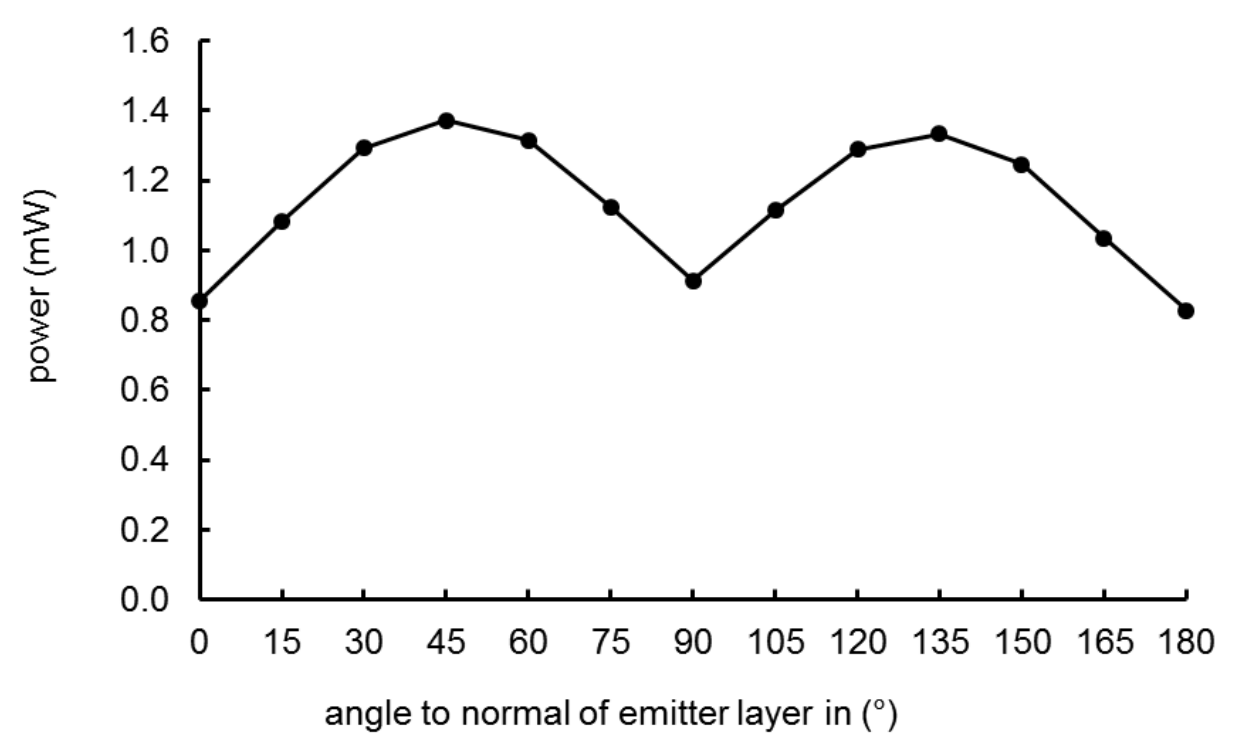

Figure 6.8 : Power output of a PV-Tower with $2.5 \mathrm{~cm}$ height.

To compare the performance of conventional planar Si solar cell and the PV-Tower solar cell, we consider a conventional planar solar cell with a surface area the same as the footprint of the PV-Tower solar cell of $200 \mu \mathrm{m} \times 200 \mu \mathrm{m}$, and with the same 
structure of $\mathrm{n}^{+}\left(500 \mathrm{~nm}, 10^{19} / \mathrm{cm}^{3}\right) / \mathrm{p}\left(200 \mu \mathrm{m}, 10^{16} / \mathrm{cm}^{3}\right) / \mathrm{p}^{+}\left(500 \mathrm{~nm}, 10^{19} / \mathrm{cm}^{3}\right)$. Under AM1.5 $100 \mathrm{~mW} / \mathrm{cm}^{2}$ solar radiation, the strongest power output that is generated by normal incident is found to be $6.85 \times 10^{-3} \mathrm{~mW}$. This translates to IF power of $125.0,133.3$, and 120.7 at $\theta=0^{\circ}, 90^{\circ}$, and $180^{\circ}$, respectively. The results mean that for the same planar area occupied, the PV-Tower solar cell can deliver power output that is more than 100 times larger as compared to the planar solar cell. Simple PV-Tower structure with $200 \times 200 \mu \mathrm{m}^{2}$ footprint and $2.5 \mathrm{~cm}$ high may yield power per footprint area of $2 \mathrm{~W} / \mathrm{cm}^{2}$ compared to $17 \mathrm{~mW} / \mathrm{cm}^{2}$ for conventional planar structure. The PV-Tower solar cell with the simulated geometry has IF area of 501.0 as shown in Fig. 6.4. The $\mathrm{IF}_{\text {power }} / \mathrm{IF}_{\text {area }}$ is calculated to be only $\approx 0.25$ since only one surface has been illuminated under the simulated directional light. Based on one directional light illumination, the back surface $\left(\theta=180^{\circ}\right)$ has the lowest $\mathrm{IF}_{\text {power }}$ of 120.7 compared to the other surfaces. Assuming an ambient with totally diffuse light, the total $\mathrm{IF}_{\text {power }}$ will be at least $4 \times \mathrm{IF}_{\text {power }}\left(\theta=180^{\circ}\right)$ or 482.8 . This will yield a ratio of $\mathrm{IF}_{\text {power }} / \mathrm{IF}_{\text {area }}>0.96$, which is very close to 1 , implying that almost the entire surface area of the PV-Tower solar cell is active in harnessing incident solar energy. 


\subsubsection{Effect of series resistance on the scaling of PV-Tower solar cell}

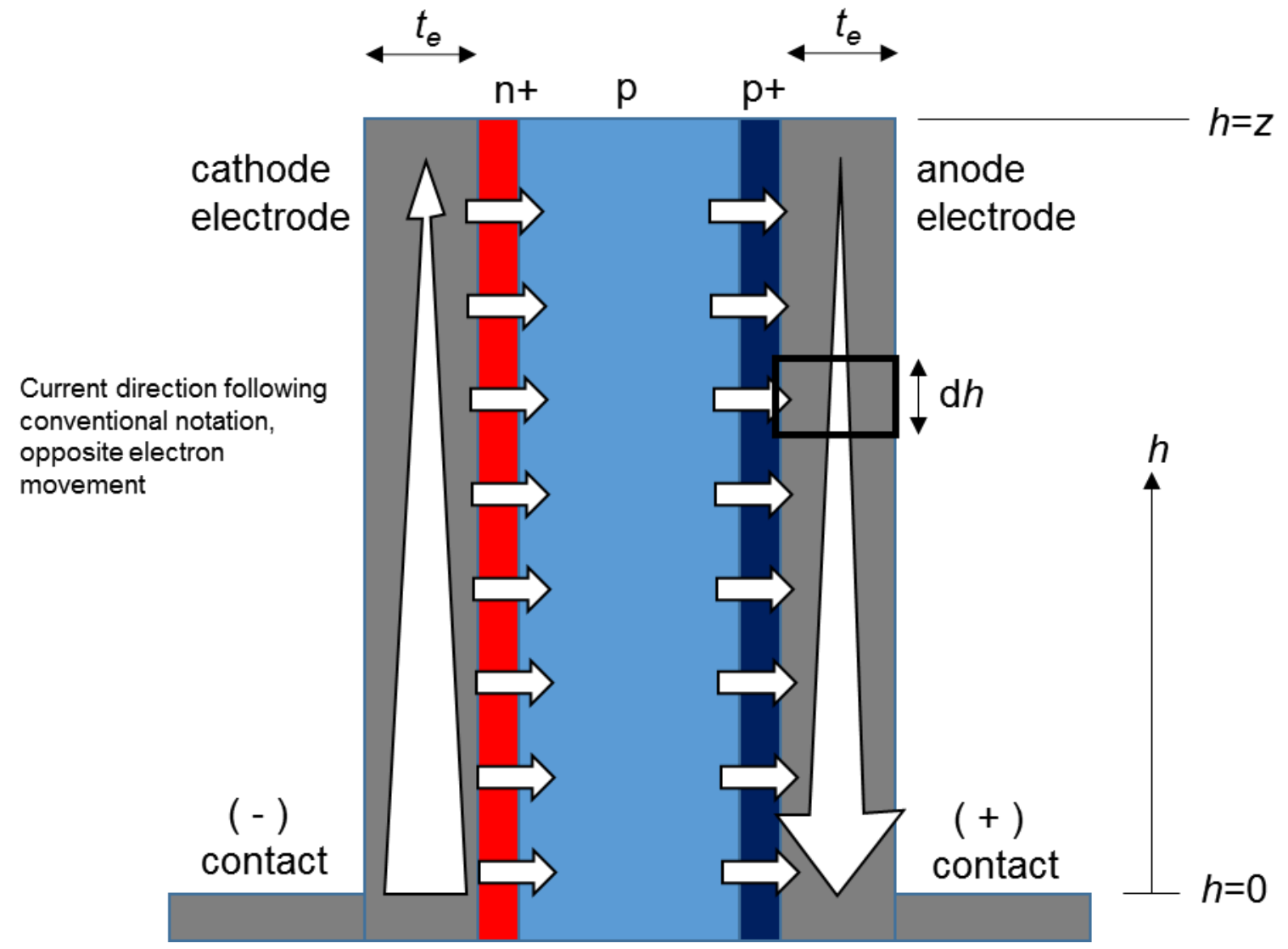

Figure 6.9 : Current flow through the metal grids on the side walls of PV-Tower.

Another important factor to consider, in the height scaling of PV-Tower solar cell to achieve higher power output, is the increase in series resistance losses for taller towers. Figure 6.9 shows the current flow through the metal grids on the side walls of a PV-Tower with a height of $z$. At the top of the anode electrode, referring to Fig. 6.9, $h=z$, the current is small since it is mainly collected from the top section of the tower. The current will increase progressively as $h$ decreases since there will be a large segment of the tower generating photocurrent and contributing to the current flow towards the bottom of the tower. At the base of the anode when $h=0$, the 
current magnitude is at its largest and is the same as the total current output of the entire tower Itower. The same variation of current also happens in the cathode electrode, with the current flowing in the opposite direction.

We assume that the light illumination is uniform on the side walls of the tower such that the current generated is constant for any given small segment of the tower with the same height $d h$. In this way, the current collected in the electrode at a given height, $I(h)$, will vary linearly with the height $h$ and is described by Eq 6.1,

$$
I(h)=I_{\text {tower }}\left(1-\frac{h}{z}\right)
$$

The ohmic losses for transport inside the semiconductors have already been taken into consideration in the 2D device numerical simulation. However, the ohmic losses in the metal grids have not been accounted for since they were assumed to be a perfect conductor in the simulation. The losses arising from the metal grid on the two sides of the PV-Tower solar cell can be significant, especially for taller towers since the grids will have a length in the range of millimeter or centimeter. The resistance $d R$ of an infinitesimal section of the electrode with a length $d h$ is described by Eq. 6.2 .

$$
d R=\frac{\rho_{e} d h}{w_{e} t_{e}}
$$

where $\rho_{e}$ is the electrode resistivity, and $w_{e}$ and $t_{e}$ are the width and thickness of the electrode. The ohmic loss $d P_{\text {loss }}$ associated with the section $d h$ of the electrode is given by $R(h) d R$, and the total power loss $P_{\text {loss }}$ can be calculated by integrating 
the power loss in each section over the entire height of the tower, from which we obtain

$$
P_{\text {loss }}=\frac{I_{\text {tower }}^{2}}{3} \frac{\rho_{e}}{w_{e} t_{e}} z
$$

Since the total current generated by the PV-Tower solar cell $I_{\text {tower }}$ is dependent on $z$, a more useful variable to study the effect of height scaling on the power loss is the current per unit length, defined as $i_{\text {tower }}=I_{\text {tower }} / z$, which is a constant for a given PV-Tower solar cell. Using Eq 6.3 and expressing in terms of $i_{\text {tower, }}$ the total power loss is given by,

$$
P_{\text {loss }}=\frac{i_{\text {tower }}^{2}}{3} \frac{\rho_{e}}{w_{e} t_{e}} z^{3}
$$

We notice that the power loss scales strongly with the tower height at a rate of $z$ cube. This is analogues to power losses associated with finger grid length that also has a cubic length functional dependence [68]. To have a more quantitative appreciation of the design constrains and the optimization of the device, we shall consider a PV-Tower solar cell with a square cross section of $200 \mu \mathrm{m} \times 200 \mu \mathrm{m}$, and with the light incident from all directions. Considering the maximum power point of the PV characteristic, we deduced from our previous device simulation that the current generated per unit height of the solar cell is $i_{\text {tower }}=2.5 \mathrm{~mA} / \mathrm{cm}$ and the voltage at maximum power point $V_{m p}=0.56 \mathrm{~V}$. We assume the use of silver electrode with resistivity $\rho_{e}=3 \times 10^{-8} \Omega \mathrm{m}$ [68], width $w_{e}=10 \mu \mathrm{m}$ and thickness $t_{e}=$ $0.2 \mu \mathrm{m}$. We further assume that the anode and cathode electrodes have the same geometry. We calculated the output power generated by the PV-Tower solar cell (i) 
in the ideal case without grid resistance, (ii) taking into account grid resistance losses. The power loss associated with the grid resistance is calculated, and all the results are displayed in Fig. 6.10 as a function of the tower height $z$.

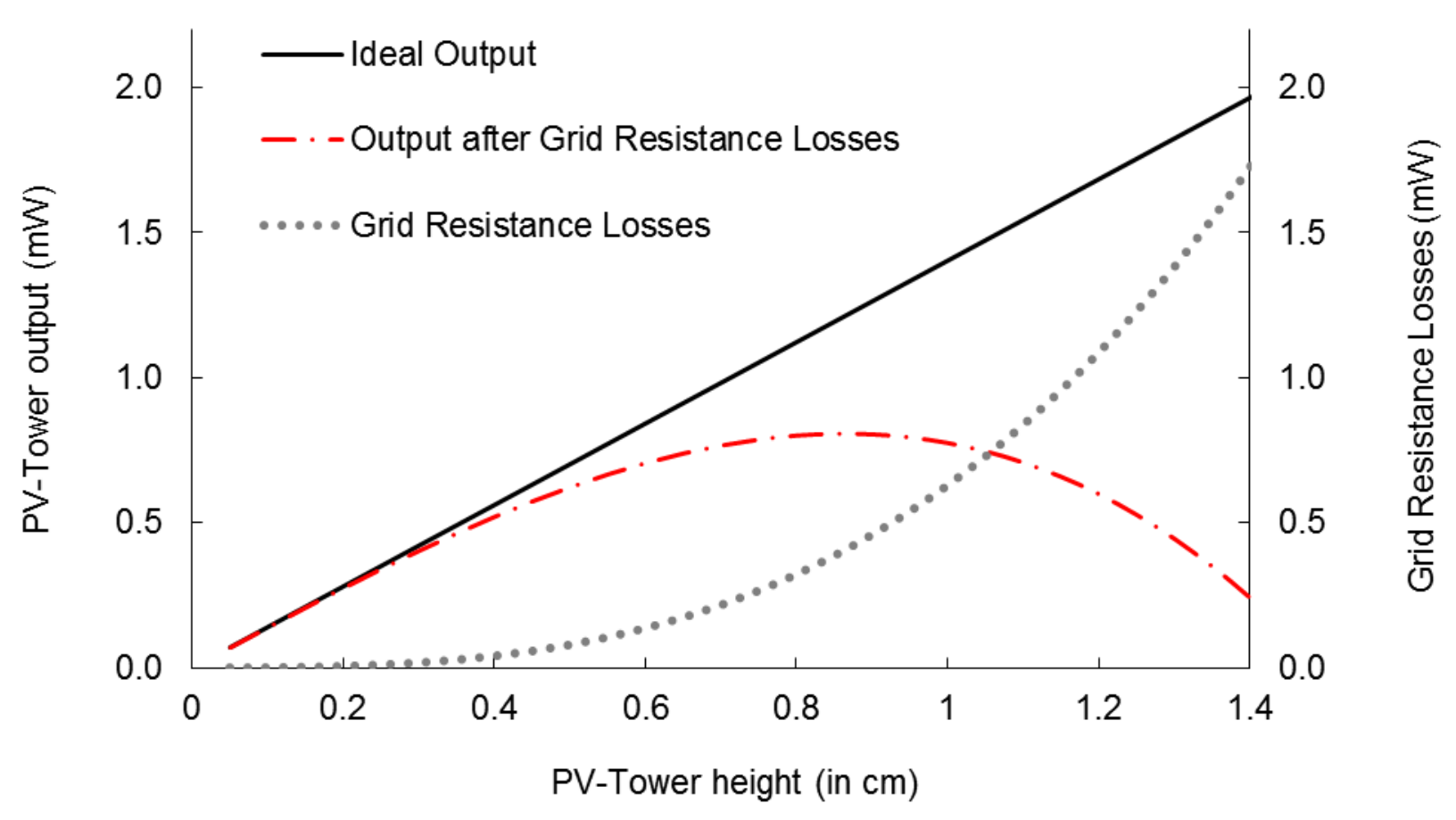

Figure 6.10 : Influence of PV-Tower height on its power output, with a tower cross section of $200 \mu \mathrm{m} \times 200 \mu \mathrm{m}$.

We notice that the power output of the ideal PV-Tower solar cell scales linearly with the height $z$, which is expected as there will be a proportional increase in the active surface area with the tower height. When we further consider the ohmic loss due to the electrode resistance which scales as a functional of $z^{3}$ as seen in Fig. 6.10, the overall power output is further reduced particularly at large $z$ and exhibits an optimum point with an optimum height $z$ of $\sim 1 \mathrm{~cm}$. Therefore, the ohmic loss 
associated with the electrodes, which is significant for the taller tower, put a limit on the maximum power output that can be achieved for a tall PV Tower solar cell.

As explained previously, a figure of merit for the design of the PV-Tower solar cell is the relative values of the area improvement factor $\left(\mathrm{IF}_{\text {area}}\right)$ and the power improvement factor (IF power). Ideally the power improvement factor should scale with the area improvement factor, however, due to ohmic losses, it is expected that $\mathrm{IF}_{\text {power }}$ will not improve proportionally with $\mathrm{IF}_{\text {area. }}$ In Fig 6.11 , we calculate and plot IF area and IF power as a function of the tower height $z$ for a PV-Tower cell with a 200 $\mu \mathrm{m} \times 200 \mu \mathrm{m}$ square cross section. It can be seen that while $\mathrm{IF}_{\text {area }}$ increases linearly with $z, \mathrm{IF}_{\text {power }}$ is limited at higher $z$, and exhibits a maximum of 117.6 at $z \approx 1 \mathrm{~cm}$. At this optimum point, the power generated by the PV-Tower solar cell is $0.8 \mathrm{~mW}$, which is 117.6 times larger than that generated by a planar solar cell with $6.85 \mu \mathrm{W}$ occupying an area of $200 \mu \mathrm{m} \times 200 \mu \mathrm{m}$ that is the same as the footprint of the PVTower cell. As can be seen, despite the limit imposed on IF $_{\text {power, }}$ the improvement in the power output by a factor of more than 100 is still a very remarkable result, and it validates the use of PV-Tower solar cell to deliver high power over a small footprint.

Besides metal electrodes, it is possible to consider the use of transparent conducting oxide for the PV-Tower solar cell that can alleviate shadowing losses and assist in the current collection from Si. We can also consider incorporating low cost plastic lenses into the PV-Tower solar cell to enhance the capturing of light 
onto the cell. For practical solar cell applications that require output voltage in the range of several volts, we can have multiple PV-Tower solar cells connected in series to boost the output voltage. Similarly, they can be connected in parallel to increase the output current generated.

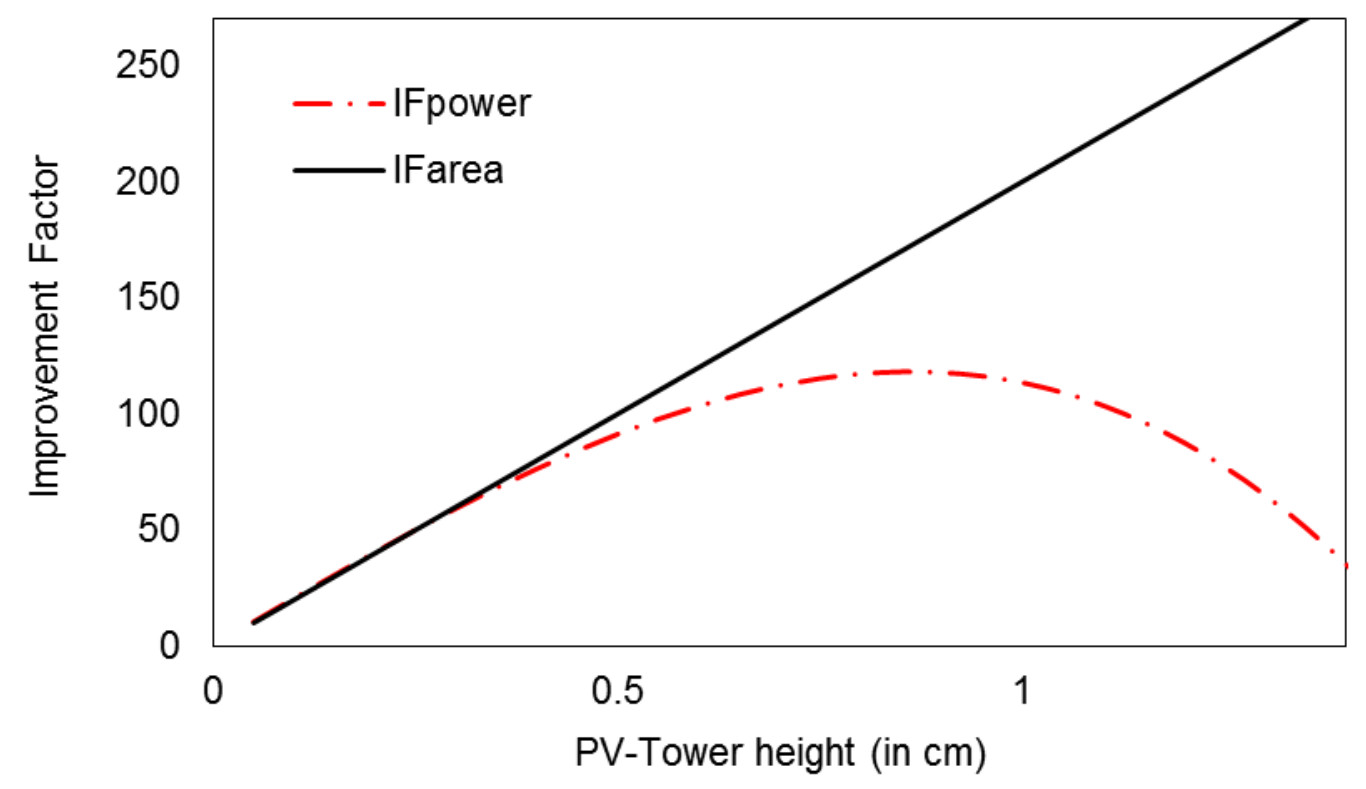

Figure 6.11 : The area and power improvement factors as a function of tower height for a PV-Tower with $200 \mu \mathrm{m} \times 200 \mu \mathrm{m}$ square cross section.

\subsection{PV-Tower with Si Heterojunction Solar Cell}

We can also demonstrate PV-Tower concept by adopting Si heterojunction solar cell structure as shown in Fig. 6.12. The use of n-Si/PEDOT:PSS allow low temperature formation of rectifying junction for the PV-Tower. The front side $(\theta=$ $0^{\circ}$ ) adopt the Front Junction with ARC compensated layer (FJ-ARC) structure which offers higher power conversion efficiency compared to conventional $n$ Si/PEDOT:PSS structure as we have demonstrated in Chapter 4 . Wafer with n- 
type doping is used in the simulation with doping concentration $\left(N_{D}\right)$ of $10^{16} / \mathrm{cm}^{3}$, and $200 \mu \mathrm{m} \times 200 \mu \mathrm{m}$ square cross section.

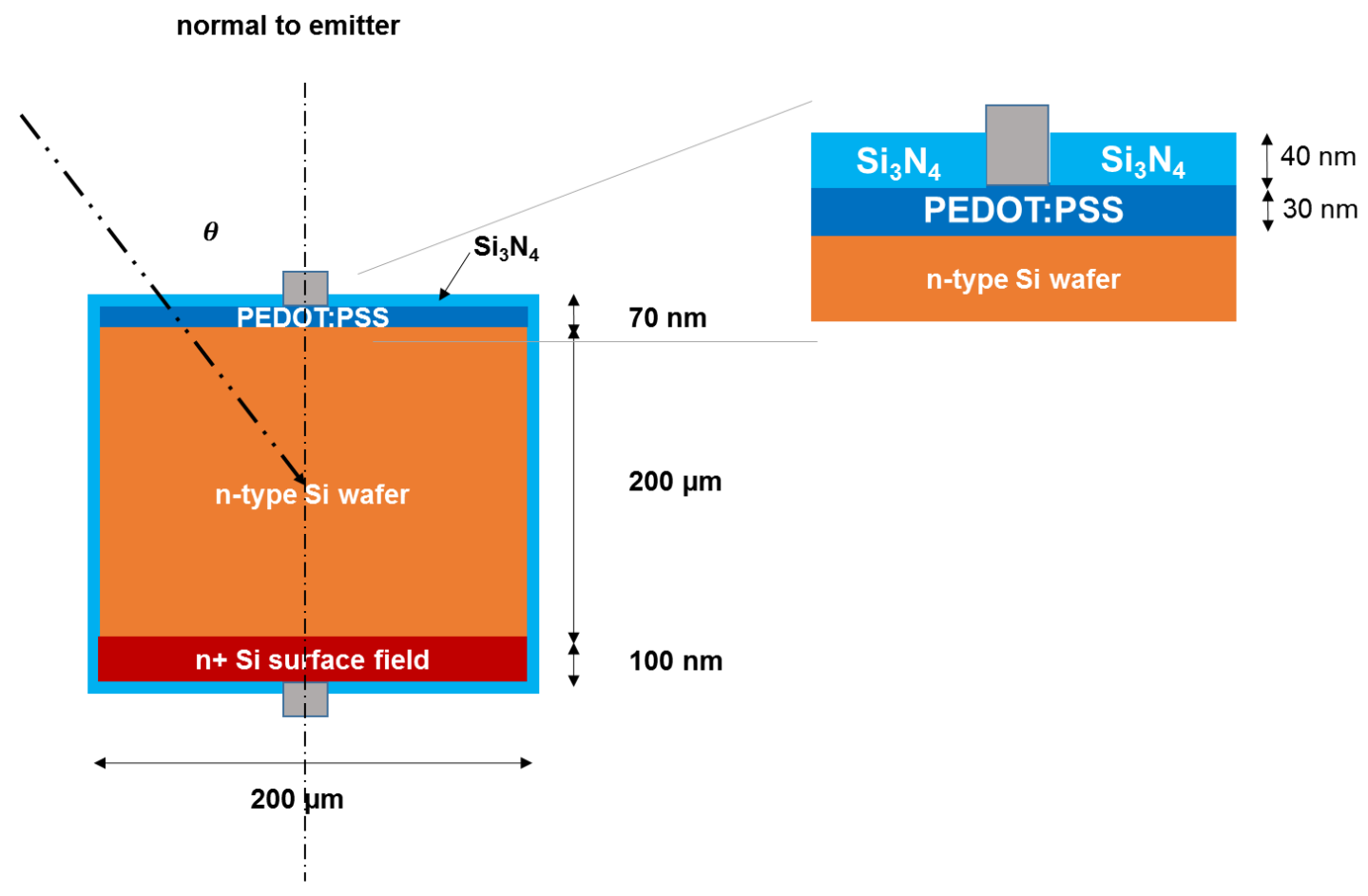

Figure 6.12 : PV-Tower with n-Si/PEDOT:PSS heterojunction solar cell.

The output power of the Si heterojunction solar cell with $2.5 \mathrm{~cm}$ height at different illumination direction is shown in Fig. 6.13. It can be seen that the power output at $\theta=0^{\circ}$, and $180^{\circ}$ is $0.90 \mathrm{~mW}$ and $0.78 \mathrm{~mW}$ respectively. This amount to $\mathrm{IF}_{\text {power }}$ of 125.0 and 109.1 for one sided illumination, which is comparable to 125.0 and 120.7 as previously demonstrated for conventional Si solar cell. Similar to those of conventional $\mathrm{Si}$ solar cell, the $\mathrm{IF}_{\text {power }}$ will be further limited by ohmic losses in the metal grid. Despite that, this result indicates that PV-Tower with Si heterojunction solar cell also shows a promising result as previously shown for PV-Tower with conventional Si homojunction solar cell. 


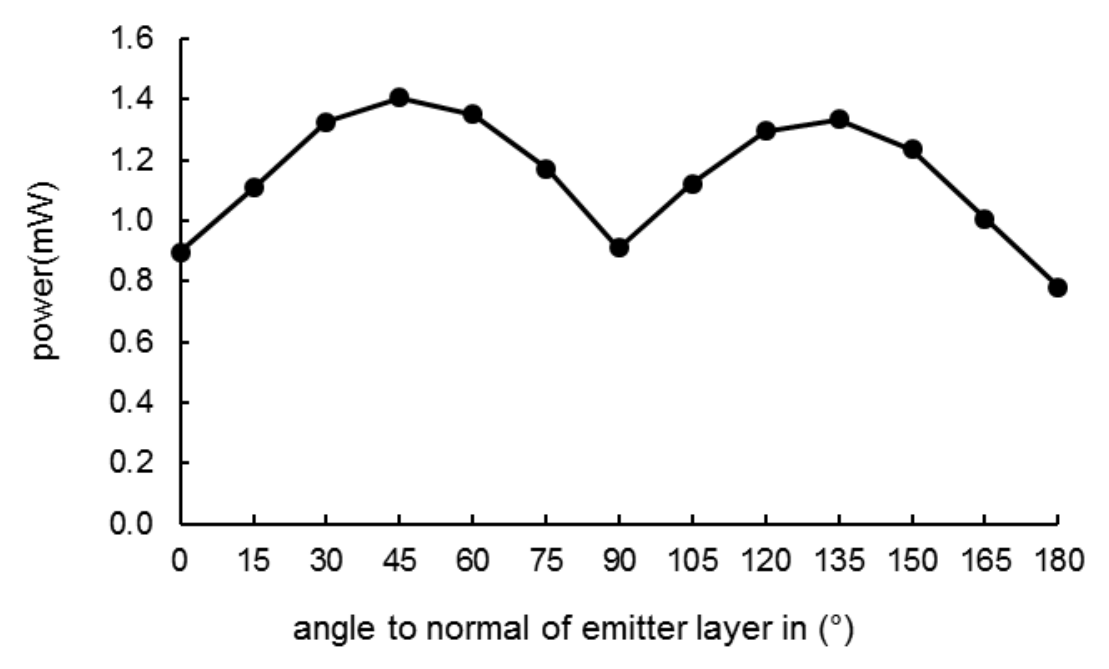

Figure 6.13 : Power output of a PV-Tower with $2.5 \mathrm{~cm}$ height that adopts Si heterojunction solar cell structure

\subsection{Conclusions}

In conclusion, we have proposed the PV-Tower cell concept as a novel device to offer high power output, owing to its large active surface area, over a small footprint. We first described in the chapter the differences between our proposed PV-Tower solar cell as compared to other solar cell concepts that offer high active surface area over a given footprint. The possible methods that can be used to fabricate the PV-Tower solar cell using industrially viable techniques have also been presented. For the quantitative study of the PV-Tower solar cell, we introduced the area improvement factor IF area and power improvement factor IF $\mathrm{F}_{\text {power }}$ as a figure of merits to assess their performance. We calculated that $\mathrm{IF}_{\text {area }}$ of 100 to 1000 is achievable for PV-Tower with a height of centimeter range, and cross section dimension of $\approx 100-200 \mu \mathrm{m}$. Subsequently, we used 2D numerical device simulation software to 
simulate the response of PV-Tower solar cell based on a conventional Si structure of $n^{+}-p-p^{+}$. We also calculated the ohmic loss associated with the electrodes on the sidewall of the PV-Tower solar cell, and it is found to scale as a function of the cubic height of the tower. Despite that, an improvement in the power output of more than 100 times has been achieved as compared to the planar solar cell having the same area. Finally, we also demonstrate with device simulation that PV-Tower cell can also adopt Si heterojunction solar cell structure with promising result. Therefore, the results suggest that the novel PV-Tower solar cell proposed has the potential to deliver high power output over a small footprint, and is ideal for energy harnessing over a limited physical area, such as in loT applications. 


\section{Chapter 7. Conclusion and Future Work}

\subsection{Conclusion}

In this project, different Si heterojunction solar cells have been simulated, fabricated and characterized. Si/PEDOT:PSS hybrid solar cell has been fabricated and characterized to understand its junction behaviour. Various device architectures based on Si/PEDOT:PSS hybrid solar cell has been simulated to optimize its performance. Si/molybdenum oxide heterojunction solar cell has been fabricated with various methods to optimize its performance. A PV-Tower solar cell concept for high power over small footprint application has been proposed and analyzed in this project.

In Chapter three, planar n-Si/PEDOT:PSS hybrid heterojunction solar cells have been fabricated with simple low-temperature solution-based processing technique. Hybrid solar cells with various $\mathrm{n}-\mathrm{Si}$ doping concentrations $\left(N_{\mathrm{D}}\right)$ from $10^{14}-10^{17} \mathrm{~cm}^{-}$

${ }^{3}$ have been investigated in terms of their junction behaviors using the reverse recovery transient (RRT) and DC I-V measurement techniques. From the RRT response, we concluded that the junction behaviour is consistent with the $n-p^{+}$ junction model and in strong contradiction with the Schottky junction model, as commonly assumed for the hybrid junction. The DC-IV measurement results of the solar cells under dark condition are also consistent with the model of diffusion, and trap-assisted recombination controlled dark current. The photovoltaic measurements of the cells under AM1.5G $100 \mathrm{~mW} / \mathrm{cm}^{2}$ illumination revealed that 
the open circuit voltage $\left(V_{o c}\right)$ increases from $0.541 \mathrm{~V}$ to $0.610 \mathrm{~V}$, while the short circuit current $\left(J_{\mathrm{sc}}\right)$ density decreases from 24.6 to $20.6 \mathrm{~mA} / \mathrm{cm}^{2}$ as $N_{D}$ increases from $10^{14}$ to $10^{17} \mathrm{~cm}^{-3}$. A maximum power conversion efficiency (PCE) of $9.8 \%$ has been obtained for the solar cell with Si doping concentration of $N_{D}=10^{16} \mathrm{~cm}^{-3}$.

In Chapter four, four different solar cell device architectures have been investigated, which include the conventional front junction (FJ) cell, back junction (BJ) cell, interdigitated back contact (IBC) cell and FJ structure with ARC compensated layer (FJ-ARC) cell. It has been found that planar BJ cell in which the PEDOT:PSS layer is located on the back surface of the Si layer can yield $J_{s c}$ of $32.1 \mathrm{~mA} / \mathrm{cm}^{2}$ and PCE of $18.8 \%$ under an optimum Si thickness of $40 \mu \mathrm{m}$. These are higher as compared to $\mathrm{Jsc}_{\mathrm{sc}}$ of $27.0 \mathrm{~mA} / \mathrm{cm}^{2}$ and PCE of $15.4 \%$ achieved for bulk conventional FJ cell. The better performance is attributed to the elimination of parasitic absorption in PEDOT:PSS. Our simulation has also demonstrated that the parasitic absorption issue affecting the FJ cell architecture can be resolved without resorting to a more demanding BJ structure. We proposed to adopt the simpler FJ structure but reduce the thickness of the PEDOT:PSS layer to $\sim 30 \mathrm{~nm}$ to minimize parasitic absorption and introduce a layer of silicon nitride anti-reflection-coating (ARC) layer with a thickness of $\sim 40 \mathrm{~nm}$ on top. We obtained a high $J_{\mathrm{sc}}$ of 32.7 $\mathrm{mA} / \mathrm{cm}^{2}$ and PCE of $18.4 \%$ for the proposed FJ-ARC cell structure, which is almost similar to those achieved for the BJ cell. We have also simulated the IBC cell in which both the hole and electron collecting contacts are located on the back side of $\mathrm{Si}$. The device architecture is free from any contact on the front side, which thus 
eliminates shadowing and parasitic absorption, and allows excellent passivation of the solar cell in one step over a large area. The use of the IBC cell structure yields the highest PCE of $20.4 \%$ at the planar device with $40 \mu \mathrm{m}$ Si substrate.

In Chapter five, we have fabricated and optimized Si/transition metal oxide (SiTMO) heterojunction solar cell based on planar $\mathrm{n}$-Si substrate and molybdenum oxide $\left(\mathrm{MoO}_{\mathrm{x}}\right)$. We studied the influence of $\mathrm{Si}$ substrate doping concentration $\left(N_{D}\right)$ on the performance of $\mathrm{n}-\mathrm{Si} / \mathrm{MoO}$ x solar cell and found that over the range $N_{D}=10^{14}$ - $10^{16} / \mathrm{cm}^{3}$, the $V_{\text {oc }}$ increases with $N_{D}$ from $0.518 \mathrm{~V}$ to $0.554 \mathrm{~V}$. At higher doping concentration $N_{D}=10^{17} / \mathrm{cm}^{3}$, the $V_{\text {oc }}$ decreases to $0.503 \mathrm{~V}$ at, which is related to the increased ohmic shunt leakage current component. On the other hand, the $J_{s c}$ decreases with increasing $N_{D}$ due to reduced diffusion length in Si. The FF also decreases with $N_{D}$, which is related to an increased ohmic shunt current leakage that is observed under lower forward bias in the dark $J-V$ characteristic. These mechanisms render the efficiency to be 10.1 to $10.2 \%$ that is almost independent of doping concentration over the range $N_{D}=10^{14}-10^{16} / \mathrm{cm}^{3}$ before it drops to $6.9 \%$ at a higher doping concentration of $N_{D}=10^{17} / \mathrm{cm}^{3}$. It is found that using an evaporated $5 \mathrm{~nm}$ thin ITO layer on top of $\mathrm{MoO}_{x}$ as a water barrier, $\mathrm{n}-\mathrm{Si} / \mathrm{MoO}$ x solar cell with PEDOT:PSS electrode can achieve $V_{\mathrm{oc}}=0.592 \mathrm{~V}, \mathrm{FF}=67.3 \%$ and PCE $=11.3 \%$. Subsequently, we study $\mathrm{n}-\mathrm{Si} / \mathrm{MoO}_{\mathrm{x}}$ solar cell with sputtered ITO electrode with or without thin evaporated ITO as a barrier layer. We found that the highest $\mathrm{PCE}=11.6 \%$ and $\mathrm{J}_{\mathrm{sc}}=34.3 \mathrm{~mA} / \mathrm{cm}^{2}$ is observed for the solar cell without the barrier layer. Although the solar cell with the barrier layer yields $V_{\text {oc }}=0.591 \mathrm{~V}$, which is 
significantly higher than $V_{o c}=0.554 \mathrm{~V}$ for those without the barrier, however, it has lower PCE $=10.7 \%$ due to its lower $\mathrm{Jsc}_{\mathrm{sc}}$ and FF. A further introduction of native oxide at the $\mathrm{n}-\mathrm{Si} / \mathrm{MoO}_{\mathrm{x}}$ interface can improve the FF of the device with the barrier layer, and enhance the efficiency to $11.4 \%$.

In Chapter 6, we have proposed the PV-Tower solar cell concept as a novel solar cell device structure to offer high power output, owing to its large active surface area, over a small footprint. For the quantitative study of the PV-Tower solar cell, we introduced the area improvement factor $\mathrm{IF}_{\text {area }}$ and power improvement factor $\mathrm{IF}_{\text {power }}$ as figures of merits to assess their performance. We calculated that an improvement in the active surface area by 100 to 1000 times is achievable for PVTower solar cell with a height of centimeter range, and cross section dimension of $\approx 100-200 \mu \mathrm{m}$. Subsequently, we used 2D numerical device simulation software to simulate the response of PV-Tower solar cell based on a conventional $\mathrm{Si}$ structure of $\mathrm{N}^{+}-\mathrm{P}-\mathrm{P}^{+}$. We also calculated the ohmic loss associated with the electrodes on the sidewall of the PV-Tower solar cell, and it is found to scale as a function of the cubic height of the tower. This limits the height of PV-Tower to a maximum of about $6 \mathrm{~cm}$. Nevertheless, PV-Tower solar cell at such height still can provide an improvement in the power output by more than 100 times as compared to a planar solar cell having the same footprint area. Finally, we also demonstrate with device simulation that PV-Tower cell can also adopt Si heterojunction solar cell structure with promising result. Therefore, the results suggest that the novel PV-Tower solar cell proposed has the potential to deliver high power output over a 
small footprint, and is ideal for energy harnessing over a limited physical area. This is important towards realizing energy autonomous sensor circuits for loT applications.

Overall, our study of Si/organic hybrid junction fabricated using the solution based process has demonstrated its great potential as a viable low cost solar cell with power conversion efficiency beyond $10 \%$. The performance of Si/organic hybrid solar cell has been limited by the parasitic absorption in the organic layer. Using different device architectures employing thinner organic layer or back junction structure can resolve this problem and realize high performance Si/organic hybrid solar cell. Si/transition metal oxide has also been studied due to its potential to offer a parasitic absorption free device with simple conventional front junction structure. However, understanding of the nature of heterojunction formed between Si and metal oxide is still in its infancy. Evaporation based deposition of metal oxide has been used in this study to investigate the concept, although solution based deposition technique can also have a potential to be used for the fabrication of Si/metal oxide heterojunction solar cell. In conclusion, owing to the excellent electronic properties of $\mathrm{Si}$ and low-temperature processing capability of organic or transition metal oxide materials, the Si/organic and Si/transition metal oxide heterojunction solar cells have great potential to realize low cost and high performing solar cells in the near future. Moreover, we also have proposed and analyzed PV-Tower cell concept, in which high power can be generated over a small footprint. PV-Tower cell can be realized using both conventional and $\mathrm{Si}$ 
heterojunction solar cell structure. The proposed PV-Tower cell concept is believed to be useful for emerging applications, such as for energy harvesting system in miniaturized Internet-of-Things Smart Node device. 


\subsection{Recommendations for future research}

There are several aspects of the present research work that can be further investigated.

\subsubsection{Fabrication of Si/PEDOT:PSS hybrid solar cell with FJ-ARC structure}

We have proposed and simulated Si/PEDOT:PSS hybrid solar cell based on the ARC compensated layer (FJ-ARC) structure. It is attractive due to its simple front junction structure and that its efficiency is comparable to that of the more demanding back junction structure. Future work would be to realize the FJ-ARC solar cell experimentally. A key challenge lies in the fabrication of the thin layer of PEDOT:PSS with low sheet resistivity. Thin and conductive PEDOT:PSS layer down to tens of nanometers can be fabricated by manipulating the spin coating speed, solvent post treatment, and thermal annealing [167]. It is also important to ensure sufficiently low PEDOT:PSS sheet resistivity to allow efficient lateral hole transport. For this purpose, we can consider an introduction of silver nanowire meshes [109] or transparent conducting oxide layer [191] to assist in the electrical conduction. We can also study FJ-ARC solar cell incorporated with antireflection structures, such as Si nanowire [192], nanohole [108] or nanocone [107], for light trapping.

\subsubsection{Fabrication of Si/PEDOT:PSS hybrid solar cell with IBC structure}

The patterning process required to form interdigitated layer of PEDOT:PSS and electrode metal on the back side of Si/PEDOT:PSS hybrid solar cell with 
interdigitated back contact (IBC) structure is the key to realizing high performing IBC cell structure. The pattern can be made by spin coating PEDOT:PSS layer over the entire back surface, followed by silver metal deposition to protect the intended PEDOT:PSS area [193] that serves as a hole collecting contact. The rest of the PEDOT:PSS can then be removed by oxygen plasma etching [193]. Finally, the cathode metal can be deposited on the removed surface to form electron collecting contact.

\subsubsection{Study of $\mathrm{n}$-Si/molybdenum oxide solar cell junction behaviour}

Understanding of the nature of the heterojunction formed between $\mathrm{n}-\mathrm{Si}$ and molybdenum oxide $\left(\mathrm{MoO}_{\mathrm{x}}\right)$ is still in its infancy. Since $\mathrm{MoO}_{\mathrm{x}}$ acts as $\mathrm{n}$-type semiconductor [94] and has a low conductivity of $2 \times 10^{-5} \mathrm{~S} / \mathrm{cm}$ [53], both $\mathrm{p}-\mathrm{n}$ and Schottky junction models are unlikely to be appropriate to explain the rectifying behavior of the $\mathrm{n}-\mathrm{Si} / \mathrm{MoO}_{\mathrm{x}}$ junction. Various characterization studies based on DC, AC and transient behaviours are needed to understand the nature of the heterojunction so as to optimize the solar cell performance. The effect of the $\mathrm{MoO}_{\mathrm{x}}$ deposition condition on its work function needs to be further investigated and correlated with the junction behavior and solar cell performance. Investigation of composition and structure of the deposited $\mathrm{MoO}_{x}$ layer will be conducted to reveal more information about the $\mathrm{MoO}_{x}$ layer. 


\subsubsection{Si/transition metal oxide solar cell with other materials and deposition process}

Other metal oxide materials such as vanadium pentoxide $\left(\mathrm{V}_{2} \mathrm{O}_{\mathrm{x}}\right)$ or nickel oxide

$\left(\mathrm{NiO}_{x}\right)$ are attractive alternatives to $\mathrm{MoO}_{x}$ as a hole accepting material for $\mathrm{n}$-Si since they are more commonly available and have higher conductivity. On the other hand, electron accepting materials such as aluminum-doped zinc oxide (AZO) or fluorinedoped tin oxide (FTO) can also be used concurrently to serve the function of back surface field layer for n-Si. AZO and FTO layer can also be used to replace ITO which is still commonly used for Si/transition metal oxide heterojunction solar cells. It is also interesting to study deposition of metal oxide on top of Si using solutionbased processing, which is important towards realizing lower cost large area solar cells.

\subsubsection{Si/molybdenum oxide solar cell with antireflection nanostructure}

Since molybdenum oxide $\left(\mathrm{MoO}_{\mathrm{x}}\right)$ has small molecule size, we can fabricate a conformal layer of this metal oxide around silicon nanowire to form core-sheath heterojunction structure. Previously, this was difficult to realize with large molecule organic polymer which cannot penetrate into the nanowire array. Such silicon nanowire $/ \mathrm{MoO}_{x}$ core-sheath structure can provide a more gradual change in refractive index which will yield better antireflection property, on top of good light trapping characteristic. The core-sheath structure also allows shorter collection distance for photogenerated carriers inside the nanowire. 


\subsubsection{Fabrication and application of PV-Tower cell}

Further work is needed to experimentally demonstrate PV-Tower solar cell with an industrially compatible process and apply it on small footprint application such as for Internet-of-Things Sensor Node (IoT-SN). The process can be done by fabricating the basic structure on large area Si wafer, and then cut it and process it to individual PV-Tower. The PV-Tower solar cell will be tested under a controlled environment such as illumination simulator and as well at applied to the real loTSN device.

\subsubsection{Investigation of ambient temperature effect on photovoltaic parameters}

Effect of ambient temperature on the photovoltaic parameters of solar cells is important to be studied in the future work. The study of ambient temperature effect may help to understand the effect of practical working condition on solar cell performance. Studying the effect of temperature on I-V curve may also reveal more detail on the transport and trap behaviour on Si/organic [104] and Si/TMO [99] heterojunction solar cell. 


\section{Author's publications}

\section{Journal}

1. Ari Bimo Prakoso, Lin Ke, Jianxiong Wang, Zeyu Li, Changyun Jiang, and Rusli, "Reverse recovery transient characteristic of PEDOT:PSS/n-Si hybrid organic-inorganic heterojunction," accepted 11 December 2016, Organic Electronics, vol. 42, pp. 269-274 (2017)

2. Ari Bimo Prakoso, Chenjin Lu, Zeyu Li, Changyun Jiang, and Rusli, "High efficiency planar front junction n-Si/PEDOT:PSS hybrid solar cell with ARC compensated layer," has been submitted to Energy.

3. Ari Bimo Prakoso, Zeyu Li, Chenjin Lu, Changyun Jiang, and Rusli "Design guideline for interdigitated back contact Si/organic hybrid heterojunction solar cell," has been submitted to Solar Energy.

4. Suresh Kumar Raman Pillai, Jing Wang, Yilei Wang, Md Moniruzzaman Sk, Ari Bimo Prakoso, Rusli, Mary B. Chan-Park, (2016). "Totally embedded hybrid thin films of carbon nanotubes and silver nanowires as flat homogenous flexible transparent conductors." Scientific Reports vol. 6 p.38453 (2016)

5. Hao Wang, Jianxiong Wang, Ari Bimo Prakoso, Lei Hong, Yew Heng Tan, Chuan Seng Tan, and Rusli, "High Efficiency Planar Thin-Film Si/PEDOT:PSS Hybrid Solar Cell," IEEE Journal of Photovoltaics vol. 6, p.217 (2016).

6. Jianxiong Wang, Hao Wang, Air Bimo Prakoso, Svietlana Alienor Togonal, Lei Hong, Changyun Jiang, Rusli, "High Efficiency Silicon Nanowire/organic Hybrid Solar Cell with Two-step Surface Treatment," Nanoscale, vol. 7, p.4559 (2015) 


\section{Conference}

1. Ari Bimo Prakoso, Rusli, Li Zeyu and Changyun Jiang, "Interdigitated backcontact back-junction Silicon/PEDOT:PSS hybrid solar cell," Hybrid and Organic Photovoltaic 2016, Swansea, UK, June 2016.

2. Li Zeyu, Rusli, Ari Bimo Prakoso and Pere Roca i Cabarrocas, "Si/PEDOT:PSS hybrid solar cell based on random Si nanowires in periodic Si nanoholes structure," Hybrid and Organic Photovoltaic 2016, Swansea, UK, June 2016.

3. Li Zeyu, Rusli, Ari Bimo Prakoso, Hong Lei and Pere Roca i Cabarrocas, "12.5\% Silicon Nanohole/PEDOT:PSS hybrid solar cell with simple solution based surface treatment", 32nd European Photovoltaic Solar Energy Conference and Exhibition (EU PVSEC 2016), Germany, June 2016.

4. H. Wang, J. X. Wang, Ari Bimo Prakoso, and Rusli, "Thin film Silicon Nanowire/PEDOT:PSS Hybrid Solar Cells with Surface Treatment," Collaborative Conference on $3 D$ and Materials Research (CC3DMR), Seoul, South Korea, 20 Jun 2016 (invited).

5. J. X. Wang, Yu Mingbin, Ari Bimo Prakoso and Rusli, "High efficiency hybrid solar cell based on the periodic nanopillars," CMOS Emerging Technologies, Montreal, Canada, May 2016. (invited).

6. J. X. Wang, Rusli, H. Wang, A. B. Prakoso, S. A. Togonal, "High Efficiency Hybrid SiNW Solar Cell With Surface Passivation," Collaborative Conference on 3D and Materials Research (CC3DMR), Busan, South Korea, 15 Jun 2015 (invited). 
7. J. X. Wang, H. Wang, A. B. Prakoso, S. A. Togonal, and Rusli, "High Efficiency Silicon Nanowire/Organic Hybrid Solar Cell with Surface Treatment," Energy Materials Nanotechnology East Meeting, Beijing China, 20 Apr 2015 (invited).

8. J. X. Wang, H. Wang, S. A. Togonal, A. B. Prakoso, L. Hong, Rusli, "Flexible Hybrid SiNW/PEDOT:PSS Solar Cell," BIT's 4th Annual World Congress of Nano Science \& Technology, Qingdao, China, 29 Oct 2014 (invited).

\section{Patents filed}

1. Rusli, Ari Bimo Prakoso, Wang Jianxiong, Wang Hong, "PV-Tower as a Compact, Efficient, Diffuse Light Harvester for Energy Autonomous Internet of Things Smart Node“ SG Patent Application no.10201607276X filing date: 1 September 2016 


\section{Bibliography}

[1] IEA, "Key World Energy Statistics," Available:

https://www.iea.org/publications/freepublications/publication/KeyWorld2016.pdf, 2016.

[2] S. Shafiee and E. Topal, "When will fossil fuel reserves be diminished?," Energy Policy, vol. 37, pp. 181-189, 2009.

[3] IPCC, "Climate Change 2014: Synthesis Report. Contribution of Working Groups I, II and III to the Fifth Assessment Report of the Intergovernmental Panel on Climate Change [Core Writing Team, R.K. Pachauri and L.A. Meyer (eds.)]," IPCC, Geneva, Switzerland, p. 151, 2014.

[4] M. I. Hoffert, K. Caldeira, A. K. Jain, E. F. Haites, L. D. D. Harvey, S. D. Potter, M. E. Schlesinger, S. H. Schneider, R. G. Watts, T. M. L. Wigley, and D. J. Wuebbles, "Energy implications of future stabilization of atmospheric CO2 content," Nature, vol. 395, pp. 881-884, 1998.

[5] M. Höök and X. Tang, "Depletion of fossil fuels and anthropogenic climate change-A review," Energy Policy, vol. 52, pp. 797-809, 2013.

[6] H.-M. Füssel, "An updated assessment of the risks from climate change based on research published since the IPCC Fourth Assessment Report," Climatic Change, vol. 97, pp. 469-482, 2009.

[7] IEA, "Technology Roadmap: Solar Photovoltaic Energy, 2014 Edition," www.iea.org, 2014.

[8] V. Fthenakis and H. C. Kim, "Land use and electricity generation: A life-cycle analysis," Renewable and Sustainable Energy Reviews, vol. 13, pp. 1465-1474, 2009.

[9] N. S. Lewis, "Powering the Planet," MRS BULLETIN, vol. 32, pp. 808-820, 2007.

[10] REN21, "Renewables 2016 Global Status Report," (Paris: REN21 Secretariat), 2016.

[11] M. A. Green, K. Emery, Y. Hishikawa, W. Warta, and E. D. Dunlop, "Solar cell efficiency tables (version 43)," Progress in Photovoltaics: Research and Applications, vol. 22, pp. 19, 2014.

[12] J. Zhao, A. Wang, P. P. Altermatt, S. R. Wenham, and M. A. Green, "24\% Efficient PERL Silicon Solar Cell, Recent Improvements in High Efficiency Silicon Cell Research," Solar Energy Materials and Solar Cells, vol. 41-42, pp. 87-89, 1996.

[13] M. A. Green, "The path to $25 \%$ silicon solar cell efficiency: History of silicon cell evolution," Progress in Photovoltaics: Research and Applications, vol. 17, pp. 183-189, 2009.

[14] W. Shockley and H. J. Queisser, "Detailed Balance Limit of Efficiency of p-n Junction Solar Cells," Journal of Applied Physics, vol. 32, p. 510, 1961.

[15] T. Saga, "Advances in crystalline silicon solar cell technology for industrial mass production," NPG Asia Materials, vol. 2, pp. 96-102, 2010.

[16] G. A. Chamberlain, "Organic Solar Cells: A Review," Solar Cells, vol. 8, pp. 47-83, 1983.

[17] H. Hoppe and N. S. Sariciftci, "Organic solar cells: An overview," Journal of Materials Research, vol. 19, pp. 1924-1945, 2011.

[18] J. Nelson, "Organic Photovoltaic Films," Current Opinion in Solid State and Materials Science, vol. 6, pp. 87-95, 2002.

[19] J.-M. Nunzi, "Organic photovoltaic materials and devices," C.R. Physique, vol. 3, pp. 523542, 2002.

[20] P. Peumans, A. Yakimov, and S. R. Forrest, "Small molecular weight organic thin-film photodetectors and solar cells," Journal of Applied Physics, vol. 93, p. 3693, 2003. 
[21] D. Wohrle and D. Meissner, "Organic Solar Cells," Advanced Materials, vol. 3, pp. 129 138, 1991.

[22] E. Bundgaard and F. Krebs, "Low band gap polymers for organic photovoltaics," Solar Energy Materials and Solar Cells, vol. 91, pp. 954-985, 2007.

[23] S. Gunes, H. Neugebauer, and N. S. Sariciftci, "Conjugated Polymer-Based Organic Solar Cells," Chem. Rev. , vol. 107, pp. 1324-1338, 2007.

[24] B. C. Thompson and J. M. Frechet, "Polymer-fullerene composite solar cells," Angew Chem Int Ed Engl, vol. 47, pp. 58-77, 2008.

[25] P. E. Shaw, A. Ruseckas, and I. D. W. Samuel, "Exciton Diffusion Measurements in Poly(3hexylthiophene)," Advanced Materials, vol. 20, pp. 3516-3520, 2008.

[26] L. He, Rusli, C. Jiang, H. Wang, and D. Lai, "Simple Approach of Fabricating High Efficiency Si Nanowire/Conductive Polymer Hybrid Solar Cells," IEEE Electron Device Letters, vol. 32, pp. 1406-1408 2011.

[27] D. Zielke, A. Pazidis, F. Werner, and J. Schmidt, "Organic-silicon heterojunction solar cells on n-type silicon wafers: The BackPEDOT concept," Solar Energy Materials and Solar Cells, vol. 131, pp. 110-116, 2014.

[28] S. Jäckle, M. Mattiza, M. Liebhaber, G. Brönstrup, M. Rommel, K. Lips, and S. Christiansen, "Junction formation and current transport mechanisms in hybrid $\mathrm{n}$ Si/PEDOT:PSS solar cells," Scientific Reports, vol. 5, p. 13008, 2015.

[29] X. Shen, Y. Zhu, T. Song, S. Lee, and B. Sun, "Hole electrical transporting properties in organic-Si Schottky solar cell," Applied Physics Letters, vol. 103, p. 013504, 2013.

[30] Y. Zhu, T. Song, F. Zhang, S. Lee, and B. Sun, "Efficient organic-inorganic hybrid Schottky solar cell: The role of built-in potential," Applied Physics Letters, vol. 102, p. 113504, 2013.

[31] K. A. Nagamatsu, S. Avasthi, J. Jhaveri, and J. C. Sturm, "A 12\% Efficient Silicon/PEDOT:PSS Heterojunction Solar Cell Fabricated at < 100 ॰C," IEEE Journal of Photovoltaics, vol. 4, pp. 260-264, 2014.

[32] J. P. Thomas and K. T. Leung, "Defect-Minimized PEDOT:PSS/Planar-Si Solar Cell with Very High Efficiency," Adv.Funct.Matter, vol. 24, pp. 4978-4985, 2014.

[33] R. Liu, S. T. Lee, and B. Sun, "13.8\% Efficiency hybrid Si/organic heterojunction solar cells with $\mathrm{MoO} 3$ film as antireflection and inversion induced layer," Adv Mater, vol. 26, pp. 6007-12, Sep 102014.

[34] P. Yu, C.-Y. Tsai, J.-K. Chang, C.-C. Lai, P.-H. Chen, Y.-C. Lai, P.-T. Tsai, M.-C. Li, H.-T. Pan, Y.-Y. Huang, C.-I. Wu, Y.-L. Chueh, S.-W. Chen, C.-H. Du, S.-F. Horng, and H.-F. Meng, "13\% Efficiency Hybrid Organic/Silicon-Nanowire Heterojunction Solar Cell via Interface Engineering," ACS Nano, vol. 7, pp. 10780-10787, 2013.

[35] S. Cho, S. H. Park, and K. Lee, "Reflectance Study on the Metal-Insulator Transition Driven by Crystallinity Change in Poly(3,4-Ethylenedioxythiophene)/Poly(Stylenesulfonate) Films," Journal of the Korean Physical Society, vol. 47, pp. 474-478, 2005.

[36] J. Ouyang, "“Secondary doping" methods to significantly enhance the conductivity of PEDOT:PSS for its application as transparent electrode of optoelectronic devices," Displays, vol. 34, pp. 423-436, 2013.

[37] J. Y. Kim, J. H. Jung, D. E. Lee, and J. Joo, "Enhancement of electrical conductivity of poly(3,4-ethylenedioxythiophene)/poly(4-styrenesulfonate) by a change of solvents," Synthetic Metals, vol. 126, pp. 311-316, 2002.

[38] O. Bubnova, Z. U. Khan, H. Wang, S. Braun, D. R. Evans, M. Fabretto, P. Hojati-Talemi, D. Dagnelund, J. B. Arlin, Y. H. Geerts, S. Desbief, D. W. Breiby, J. W. Andreasen, R. 
Lazzaroni, W. M. Chen, I. Zozoulenko, M. Fahlman, P. J. Murphy, M. Berggren, and X. Crispin, "Semi-metallic polymers," Nat Mater, vol. 13, pp. 190-4, Feb 2014.

[39] A. S. Erickson, A. Zohar, and D. Cahen, "n-Si-Organic Inversion Layer Interfaces: A Low Temperature Deposition Method for Forming a p-n Homojunction in n-Si," Advanced Energy Materials, vol. 4, p. 1301724, 2014.

[40] M. J. Price, J. M. Foley, R. A. May, and S. Maldonado, "Comparison of majority carrier charge transfer velocities at Si/polymer and Si/metal photovoltaic heterojunctions," Applied Physics Letters, vol. 97, p. 083503, 2010.

[41] X. Zhang, D. Yang, Z. Yang, X. Guo, B. Liu, X. Ren, and S. F. Liu, "Improved PEDOT:PSS/c-Si hybrid solar cell using inverted structure and effective passivation," Sci Rep, vol. 6, p. 35091, 2016.

[42] H. D. Um, N. Kim, K. Lee, I. Hwang, J. H. Seo, and K. Seo, "Dopant-Free All-Back-Contact Si Nanohole Solar Cells Using MoOx and LiF Films," Nano Lett, vol. 16, pp. 981-987, 2016.

[43] L. Fang, S. J. Baik, and K. Su Limc, "Transition metal oxide window layer in thin film amorphous silicon solar cells," Thin Solid Films, vol. 556, pp. 515-519, 2014.

[44] B. Macco, M. F. J. Vos, N. F. W. Thissen, A. A. Bol, and W. M. M. Kessels, "Lowtemperature atomic layer deposition of MoOx for silicon heterojunction solar cells," Phys. Status Solidi RRL, vol. 9, pp. 393-396, 2015.

[45] K. Zilberberg, H. Gharbi, A. Behrendt, S. Trost, and T. Riedl, "Low-temperature, solutionprocessed $\mathrm{MoO}(\mathrm{x})$ for efficient and stable organic solar cells," ACS Appl Mater Interfaces, vol. 4, pp. 1164-8, Mar 2012.

[46] H. Oh, J. Krantz, I. Litzov, T. Stubhan, L. Pinna, and C. J. Brabec, "Comparison of various sol-gel derived metal oxide layers for inverted organic solar cells," Solar Energy Materials and Solar Cells, vol. 95, pp. 2194-2199, 2011.

[47] C. Girotto, E. Voroshazi, D. Cheyns, P. Heremans, and B. P. Rand, "Solution-processed $\mathrm{MoO}(3)$ thin films as a hole-injection layer for organic solar cells," ACS Appl Mater Interfaces, vol. 3, pp. 3244-7, Sep 2011.

[48] F. A. S. Lima, M. J. Beliatis, B. Roth, T. R. Andersen, A. Bortoti, Y. Reyna, E. Castro, I. F. Vasconcelos, S. A. Gevorgyan, F. C. Krebs, and M. Lira-Cantu, "Flexible ITO-free organic solar cells applying aqueous solution-processed V2O5 hole transport layer: An outdoor stability study," APL Materials, vol. 4, p. 026104, 2016.

[49] Z. a. Tan, L. Li, C. Cui, Y. Ding, Q. Xu, S. Li, D. Qian, and Y. Li, "Solution-Processed Tungsten Oxide as an Effective Anode Buffer Layer for High-Performance Polymer Solar Cells," The Journal of Physical Chemistry C, vol. 116, pp. 18626-18632, 2012.

[50] Y. Sun, J. H. Seo, C. J. Takacs, J. Seifter, and A. J. Heeger, "Inverted polymer solar cells integrated with a low-temperature-annealed sol-gel-derived ZnO Film as an electron transport layer," Adv Mater, vol. 23, pp. 1679-83, Apr 122011.

[51] C. Waldauf, M. Morana, P. Denk, P. Schilinsky, K. Coakley, S. A. Choulis, and C. J. Brabec, "Highly efficient inverted organic photovoltaics using solution based titanium oxide as electron selective contact," Applied Physics Letters, vol. 89, p. 233517, 2006.

[52] C. Battaglia, S. M. de Nicolás, S. De Wolf, X. Yin, M. Zheng, C. Ballif, and A. Javey, "Silicon heterojunction solar cell with passivated hole selective MoOx contact," Applied Physics Letters, vol. 104, p. 113902, 2014.

[53] J. Bullock, A. Cuevas, T. Allen, and C. Battaglia, "Molybdenum oxide MoOx: A versatile hole contact for silicon solar cells," Applied Physics Letters, vol. 105, p. 232109, 2014.

[54] J. Bullock, D. Yan, A. Cuevas, Y. Wan, and C. Samundsett, " $n$ - and p-type silicon solar cells with molybdenum oxide hole contacts," Energy Procedia, vol. 77, pp. 446 - 450, 2015. 
[55] B. A. Warneke, M. D. Scott, B. S. Leibowitz, L. Zhou, C. L. Bellew, J. A. Chediak, J. M. Kahn, B. E. Boser, and K. S. J. Pister, "An Autonomous $16 \mathrm{~mm} 3$ Solar-Powered Node for Distributed Wireless Sensor Networks," Proceedings of IEEE Sensors, vol. 1, pp. 15101515, 2002.

[56] W. Y. Toh, Y. K. Tan, W. S. Koh, and L. Siek, "Autonomous Wearable Sensor Nodes With Flexible Energy Harvesting," IEEE Sensors Journal, vol. 14, pp. 2299-2306, 2014.

[57] C. O. Mathuna, T. O'Donnell, R. V. Martinez-Catala, J. Rohan, and B. O'Flynn, "Energy Scavenging for Long-Term Deployable Wireless Sensor Networks," Talanta, vol. 75, pp. 613-23, 2008.

[58] M. Danesh and J. R. Long, "An Autonomous Wireless Sensor Node Using a Solar Cell Antenna for Solar Energy Harvesting," IEEE Transactions on Microwave Theory and Techniques, vol. 59, pp. 3546-3555, 2011.

[59] A. Teran, M. Dejarld, J. Hwang, W. Lim, J. Wong, D. Blaauw, Y. Lee, J. Millunchick, and J. Phillips, "Indoor Photovoltaic Energy Harvesting for mm-scale Systems," presented at the 72nd Device Research Conference, Santa Barbara, 2014.

[60] A. S. Teran, W. Lim, Y. Lee, and J. D. Phillips, "AlGaAs Photovoltaics for Indoor Energy Harvesting in mm-Scale Wireless Sensor Nodes," IEEE Transactions on Electron Devices, vol. 62, pp. 2170-2175, 2015.

[61] C. Botteron, D. Briand, B. Mishra, G. Tasselli, P. Janphuang, F. J. Haug, A. Skrivervik, R. Lockhart, C. Robert, N. F. de Rooij, and P. A. Farine, "A Low-Cost UWB Sensor Node Powered by a Piezoelectric Harvester or Solar Cells," Sensors and Actuators A: Physical, vol. 239, pp. 127-136, 2016.

[62] H. O. Jian Lu, Toshihiro Itoh, Takeshi Harada, and Ryutaro Maeda, "Toward the World Smallest Wireless Sensor Nodes With Ultralow Power Consumption," IEEE Sensors Journal, vol. 14, pp. 2035-2041, 2014.

[63] C. Alippi and C. Galperti, "An Adaptive System for Optimal Solar Energy Harvesting in Wireless Sensor Network Nodes," IEEE Transactions on Circuits and Systems I: Regular Papers, vol. 55, pp. 1742-1750, 2008.

[64] M. K. Stojcev, M. R. Kosanovic, and L. R. Golubovic, "Power management and energy harvesting techniques for wireless sensor nodes," presented at the 9th International Conference on Telecommunication in Modern Satellite, Cable, and Broadcasting Services, 2009.

[65] A. Sinha and A. Chandrakasan, "Dynamic power management in wireless sensor networks," IEEE Design \& Test of Computers, vol. 18, pp. 62-74, 2001.

[66] S. Tarannum, "Energy Conservation Challenges in Wireless Sensor Networks: A Comprehensive Study," Wireless Sensor Network, vol. 02, pp. 483-491, 2010.

[67] F. D. Rossi, T. M. Brown, and T. Pontecorvo, "Flexible photovoltaics for light harvesting under LED lighting," presented at the IEEE 15th International Conference on Environment and Electrical Engineering (EEEIC), 2015.

[68] S. Bowden and C. Honsberg. (2012). PVCDROM. Available: http://pveducation.org/pvcdrom

[69] M. A. Green, "Third Generation Photovoltaics: Ultra-high Conversion Efficiency at Low Cost," Progress in Photovoltaics: Research and Applications, vol. 9, pp. 123-135, 2001.

[70] UNSW, "ARC Photovoltaics Centre of Excellence Annual Report 2014," 2014.

[71] G. Conibeer, "Third-generation photovoltaics," Materials Today, vol. 10, pp. 42-50, 2007.

[72] M. A. Green, "Third Generation Photovoltaics: solar cells for 2020 and beyond," Physica E, vol. 14, pp. 65-70, 2002. 
[73] NREL, "Best Research-Cell Efficiencies," Available:

http://www.nrel.gov/pv/assets/images/efficiency chart.jpg, 2017.

[74] K. Corporation, "World's Highest Conversion Efficiency of $26.33 \%$ Achieved in a Crystalline Silicon Solar Cell - A World First in a Practical Cell Size -," Press release, NEDO and Kaneka corporation, 2016.

[75] Y. Zhou and K. Zhu, "Perovskite Solar Cells Shine in the "Valley of the Sun"," ACS Energy Letters, vol. 1, pp. 64-67, 2016.

[76] J. You, L. Meng, T. B. Song, T. F. Guo, Y. M. Yang, W. H. Chang, Z. Hong, H. Chen, H. Zhou, Q. Chen, Y. Liu, N. De Marco, and Y. Yang, "Improved air stability of perovskite solar cells via solution-processed metal oxide transport layers," Nat Nanotechnol, vol. 11, pp. 7581, Jan 2016.

[77] T. Leijtens, G. E. Eperon, S. Pathak, A. Abate, M. M. Lee, and H. J. Snaith, "Overcoming ultraviolet light instability of sensitized $\mathrm{TiO}(2)$ with meso-superstructured organometal tri-halide perovskite solar cells," Nat Commun, vol. 4, p. 2885, 2013.

[78] R. F. Pierret, Semiconductor Device Fundamentals, 1996.

[79] M. A. Green, "Crystalline Silicon Photovoltaic Cell," Advanced Materials, vol. 13, pp. 1019-1022, 2001.

[80] M. A. Green, "Crystalline and thin-film silicon solar cells: state of the art and future potential," Solar Energy, vol. 74, pp. 181-192, 2003.

[81] A. Shah, J. Meier, A. Buechel, U. Kroll, J. Steinhauser, F. Meillaud, H. Schade, and D. Dominé, "Towards very low-cost mass production of thin-film silicon photovoltaic (PV) solar modules on glass," Thin Solid Films, vol. 502, pp. 292-299, 2006.

[82] D. E. Carlson and C. R. Wronski, "Amorphous silicon solar cell," Applied Physics Letters, vol. 28, pp. 671-673, 1976.

[83] C. H. Lee, D. R. Kim, I. S. Cho, N. William, Q. Wang, and X. Zheng, "Peel-and-stick: fabricating thin film solar cell on universal substrates," Sci Rep, vol. 2, p. 1000, 2012.

[84] H. Sai, T. Matsui, T. Koida, K. Matsubara, M. Kondo, and S. Sugiyama, "Triple-junction thin-film silicon solar cell fabricated on periodically textured substrate with a stabilized efficiency of 13.6\%," Applied Physics Letters, vol. 106, p. 213902, 2015.

[85] Z. Shen, T. Masuda, H. Takagishi, K. Ohdaira, and T. Shimoda, "Fabrication of high-quality amorphous silicon film from cyclopentasilane by vapor deposition between two parallel substrates," Chem Commun (Camb), vol. 51, pp. 4417-20, Mar 142015.

[86] G. Garcia-Belmonte, P. P. Boix, J. Bisquert, M. Sessolo, and H. J. Bolink, "Simultaneous determination of carrier lifetime and electron density-of-states in P3HT:PCBM organic solar cells under illumination by impedance spectroscopy," Solar Energy Materials and Solar Cells, vol. 94, pp. 366-375, 2010.

[87] S. Dimitrov, B. Schroeder, C. Nielsen, H. Bronstein, Z. Fei, I. McCulloch, M. Heeney, and J. Durrant, "Singlet Exciton Lifetimes in Conjugated Polymer Films for Organic Solar Cells," Polymers, vol. 8, p. 14, 2016.

[88] O. V. Mikhnenko, P. W. M. Blom, and T.-Q. Nguyen, "Exciton diffusion in organic semiconductors," Energy Environ. Sci., vol. 8, pp. 1867-1888, 2015.

[89] C. C. Chen, W. H. Chang, K. Yoshimura, K. Ohya, J. You, J. Gao, Z. Hong, and Y. Yang, "An efficient triple-junction polymer solar cell having a power conversion efficiency exceeding 11\%," Adv Mater, vol. 26, pp. 5670-7, Aug 272014.

[90] M. Jørgensen, K. Norrman, and F. C. Krebs, "Stability/degradation of polymer solar cells," Solar Energy Materials and Solar Cells, vol. 92, pp. 686-714, 2008.

[91] S. Ruhle, A. Y. Anderson, H. N. Barad, B. Kupfer, Y. Bouhadana, E. Rosh-Hodesh, and A. Zaban, "All-Oxide Photovoltaics," J Phys Chem Lett, vol. 3, pp. 3755-64, Dec 202012. 
[92] H. Peng, A. Bikowski, A. Zakutayev, and S. Lany, "Pathway to oxide photovoltaics via band-structure engineering of SnO," APL Materials, vol. 4, p. 106103, 2016.

[93] M. Pavan, S. Rühle, A. Ginsburg, D. A. Keller, H.-N. Barad, P. M. Sberna, D. Nunes, R. Martins, A. Y. Anderson, A. Zaban, and E. Fortunato, "TiO2/Cu2O all-oxide heterojunction solar cells produced by spray pyrolysis," Solar Energy Materials and Solar Cells, vol. 132, pp. 549-556, 2015.

[94] J. Meyer, S. Hamwi, M. Kroger, W. Kowalsky, T. Riedl, and A. Kahn, "Transition metal oxides for organic electronics: energetics, device physics and applications," Adv Mater, vol. 24, pp. 5408-27, Oct 232012.

[95] S. Avasthi, K. A. Nagamatsu, J. Jhaveri, W. E. McClain, G. Man, A. Kahn, J. Schwartz, S. Wagner, and J. C. Sturm, "Double-Heterojunction Crystalline Silicon Solar Cell Fabricated at $250 \mathrm{C}$ with $12.9 \%$ Efficiency," presented at the IEEE 40th Photovoltaic Specialist Conference, PVSC 2014, Denver; US, 2014.

[96] H. Kobayashi, H. Mori, T. Ishida, and Y. Nakato, "Zinc oxide/n-Si junction solar cells produced by spray-pyrolysis method," Journal of Applied Physics, vol. 77, p. 1301, 1995.

[97] C. Battaglia, X. Yin, M. Zheng, I. D. Sharp, T. Chen, S. McDonnell, A. Azcatl, C. Carraro, B. Ma, R. Maboudian, R. M. Wallace, and A. Javey, "Hole Selective MoOx Contact for Silicon Solar Cells," Nano Lett, vol. 14, pp. 967-971, Jan 272014.

[98] M. Bivour, J. Temmler, H. Steinkemper, and M. Hermle, "Molybdenum and tungsten oxide: High work function wide band gap contact materials for hole selective contacts of silicon solar cells," Solar Energy Materials \& Solar Cells, vol. 142, pp. 34-41, 2015.

[99] L. G. Gerling, S. Mahato, A. Morales-Vilches, G. Masmitja, P. Ortega, C. Voz, R. Alcubilla, and J. Puigdollers, "Transition metal oxides as hole-selective contacts in silicon heterojunctions solar cells," Solar Energy Materials and Solar Cells, vol. 145, pp. 109-115, 2016.

[100] M. Taguchi, A. Yano, S. Tohoda, K. Matsuyama, Y. Nakamura, T. Nishiwaki, K. Fujita, and E. Maruyama, "24.7\% Record Efficiency HIT Solar Cell on Thin Silicon Wafer," IEEE Journal of Photovoltaic, vol. 4, pp. 96 - 99, JANUARY 20142015.

[101] K. Masuko, M. Shigematsu, T. Hashiguchi, D. Fujishima, M. Kai, N. Yoshimura, T. Yamaguchi, Y. Ichihashi, T. Mishima, N. Matsubara, T. Yamanishi, T. Takahama, M. Taguchi, E. Maruyama, and S. Okamoto, "Achievement of more than $25 \%$ conversion efficiency with crystalline silicon heterojunction solar cell," IEEE Journal of Photovoltaics, vol. 4, pp. 1433-1435, 2014.

[102] L. He, C. Jiang, H. Wang, D. Lai, and Rusli, "High Efficiency Planar Si/Organic Heterojunction Hybrid Solar Cells," Applied Physics Letters, vol. 100, p. 073503, 2012.

[103] L. He, C. Jiang, H. Wang, H. Lei, D. Lai, and Rusli, "11.3\% efficient planar Si-PEDOT:PSS hybrid solar cell with a thin interfacial oxide," presented at the IEEE Photovoltaic Specialists Conference, 2012.

[104] V. V. Brus, M. Zellmeier, X. Zhang, S. M. Greil, M. Gluba, A. J. Töfflinger, J. Rappich, and N. $\mathrm{H}$. Nickel, "Electrical and photoelectrical properties of P3HT/n-Si hybrid organicinorganic heterojunction solar cells," Organic Electronics, vol. 14, pp. 3109-3116, 2013.

[105] G. Kalita, S. Adhikari, H. R. Aryal, R. Afre, T. Soga, M. Sharon, W. Koichi, and M. Umeno, "Silicon nanowire array/polymer hybrid solar cell incorporating carbon nanotubes," Journal of Physics D: Applied Physics, vol. 42, p. 115104, 2009.

[106] L. He, C. Jiang, H. Wang, D. Lai, and Rusli, "Si Nanowires Organic Semiconductor Hybrid Heterojunction Solar Cells Toward 10\% Efficiency," ACS Appl Mater Interfaces, vol. 4, pp. 1704-8, 2012. 
[107] S. Jeong, E. C. Garnett, S. Wang, Z. Yu, S. Fan, M. L. Brongersma, M. D. McGehee, and Y. Cui, "Hybrid silicon nanocone-polymer solar cells," Nano Lett, vol. 12, pp. 2971-6, 2012.

[108] L. Hong, X. Wang, H. Zheng, L. He, H. Wang, H. Yu, and Rusli, "High Efficiency Silicon Nanohole/Organic Heterojunction Hybrid Solar Cell," Applied Physics Letters, vol. 104, p. 053104, 2014.

[109] T. G. Chen, B. Y. Huang, H. W. Liu, Y. Y. Huang, H. T. Pan, H. F. Meng, and P. Yu, "Flexible silver nanowire meshes for high-efficiency microtextured organic-silicon hybrid photovoltaics," ACS Appl Mater Interfaces, vol. 4, pp. 6857-64, Dec 2012.

[110] K. T. Park, H. J. Kim, M. J. Park, J. H. Jeong, J. Lee, D. G. Choi, J. H. Lee, and J. H. Choi, "13.2\% efficiency Si nanowire/PEDOT:PSS hybrid solar cell using a transfer-imprinted Au mesh electrode," Sci Rep, vol. 5, p. 12093, 2015.

[111] M. Sharma, P. R. Pudasaini, F. Ruiz-Zepeda, D. Elam, and A. A. Ayon, "Ultrathin, flexible organic-inorganic hybrid solar cells based on silicon nanowires and PEDOT:PSS," ACS Appl Mater Interfaces, vol. 6, pp. 4356-63, Mar 262014.

[112] M. L. Tsai, W. R. Wei, L. Tang, H. C. Chang, S. H. Tai, P. K. Yang, S. P. Lau, L. J. Chen, and J. H. He, "Si Hybrid Solar Cells with 13\% Efficiency via Concurrent Improvement in Optical and Electrical Properties by Employing Graphene Quantum Dots," ACS Nano, vol. 10, pp. 815-21, Jan 262016.

[113] Z. C. Holman, A. Descoeudres, L. Barraud, F. Z. Fernandez, J. P. Seif, S. DeWolf, and C. Ballif, "Current Losses at the Front of Silicon Heterojunction Solar Cells," IEEE Journal of Photovoltaic, vol. 2, pp. 7 - 15, January, 20122012.

[114] E. S. Biagioni, "The Application of Remote Sensor Technology to Assist the Recovery of Rare and Endangered Species," The International Journal of High Performance Computing Applications, vol. 16, pp. 315-324, 2002.

[115] C. Alippi, R. Camplani, C. Galperti, and M. Roveri, "A Robust, Adaptive, Solar-Powered WSN Framework for Aquatic Environmental Monitoring," IEEE Sensors Journal, vol. 11, pp. 45-55, 2011.

[116] R. Morais, S. G. Matos, M. A. Fernandes, A. L. G. Valente, S. F. S. P. Soares, P. J. S. G. Ferreira, and M. J. C. S. Reis, "Sun, Wind and Water Flow as Energy Supply for Small Stationary Data Acquisition Platforms," Computers and Electronics in Agriculture, vol. 64, pp. 120-132, 2008.

[117] D. Evans, "The Internet of Things: How the Next Evolution of the Internet Is Changing Everything," CISCO White Paper, 2011.

[118] H. O. Al-Sakran, "Intelligent Traffic Information System Based on Integration of Internet of Things and Agent Technology," International Journal of Advanced Computer Science and Applications, vol. 6, pp. 37-43, 2015.

[119] F. Zafari, I. Papapanagiotou, and K. Christidis, "Microlocation for Internet-of-ThingsEquipped Smart Buildings," IEEE Internet of Things Journal, vol. 3, pp. 96-112, 2016.

[120] C. Knight, J. Davidson, and S. Behrens, "Energy Options for Wireless Sensor Nodes," Sensors, vol. 8, pp. 8037-8066, 2008.

[121] A. Hande, T. Polk, W. Walker, and D. Bhatia, "Indoor Solar Energy Harvesting for Sensor Network Router Nodes," Microprocessors and Microsystems, vol. 31, pp. 420-432, 2007.

[122] V. Raghunathan, A. Kansal, J. Hsu, J. Friedman, and M. Srivastava, "Design Considerations for Solar Energy Harvesting Wireless Embedded Systems," presented at the Fourth International Symposium on Information Processing in Sensor Networks 2005, 2005.

[123] P. J. Verlinden, A. W. Blakers, K. J. Weber, J. Babaei, V. Everett, M. J. Kerr, M. F. Stuckings, D. Gordeev, and M. J. Stocks, "Sliver ${ }^{\circledast}$ Solar Cells: A New Thin-Crystalline Silicon 
Photovoltaic Technology," Solar Energy Materials and Solar Cells, vol. 90, pp. 3422-3430, 2006.

[124] W. J. Ready, "Nano-Manhattan: 3-D Solar Cell that Uses "Towers" to Boost Efficiency Wins International Patents," Georgia Tech Research News \& Publications, 2009.

[125] W. J. Ready, "Three dimensional multi-junction photovoltaic device," U.S. Patent \# 8,350,146, 2013.

[126] C. G. Rampley, F. T. Laver, and T. E. Wood, "Three-Dimensional Solar Cell Having Increased Efficiency," U.S. Patent \#8, 790,947, 2014.

[127] M. N. Dagli and C. Son, "Wide Angle Three-Dimensional Solar Cells," U.S. Patent Application Publication \#2015/0027518, 2015.

[128] M. Bernardi, N. Ferralis, J. H. Wan, R. Villalon, and J. C. Grossman, "Solar Energy Generation in Three Dimensions," Energy \& Environmental Science, vol. 5, pp. 68806884, 2012.

[129] Z. Liang, P. Zeng, P. Liu, C. Zhao, W. Xie, and W. Mai, "Interface Engineering To Boost Photoresponse Performance of Self-Powered, Broad-Bandwidth PEDOT:PSS/Si Heterojunction Photodetector," ACS Appl Mater Interfaces, vol. 8, pp. 19158-67, Jul 27 2016.

[130] J. Jhaveri, S. Avasthi, K. A. Nagamatsu, and J. C. Sturm, "Wide Bandgap HBT on Crystalline Silicon using Electron-Blocking PEDOT:PSS Emitter," presented at the Device Research Conference (DRC), 2013 71st Annual, Notre Dame, IN, 2013.

[131] A. Wang, J. Zhao, and M. A. Green, "24\% efficient silicon solar cells," Applied Physics Letters, vol. 57, p. 602, 1990.

[132] M. G. Walter, X. Liu, L. E. O'Leary, B. S. Brunschwig, and N. S. Lewis, "Electrical Junction Behavior of Poly(3,4-ethylenedioxythiophene) (PEDOT) Contacts to H-Terminated and CH3-Terminated p-, n-, and n+-Si(111) Surfaces," The Journal of Physical Chemistry C, vol. 117, pp. 14485-14492, 2013.

[133] F. Zhang, D. Liu, Y. Zhang, H. Wei, T. Song, and B. Sun, "Methyl/allyl monolayer on silicon: efficient surface passivation for silicon-conjugated polymer hybrid solar cell," ACS Appl Mater Interfaces, vol. 5, pp. 4678-84, 2013.

[134] W. W. He, K. J. Wu, K. Wang, T. F. Shi, L. Wu, S. X. Li, D. Y. Teng, and C. H. Ye, "Towards stable silicon nanoarray hybrid solar cells," Sci Rep, vol. 4, p. 3715, 2014.

[135] J. Li, J. Liu, C. Gao, J. Zhang, and H. Sun, "Influence of MWCNTs Doping on the Structure and Properties of PEDOT:PSS Films," International Journal of Photoenergy, vol. 2009, pp. 1-5, 2009.

[136] A. Elschner, S. Kirchmeyer, W. Lovenich, U. Merker, and K. Reuter, PEDOT: Principles and Applications of an Intrinsically Conductive Polymer, Chapter 9 p.147: CRC Press, 2011.

[137] Y.-J. Lin, B.-C. Huang, Y.-C. Lien, C.-T. Lee, C.-L. Tsai, and H.-C. Chang, "Capacitancevoltage and current-voltage characteristics of Au Schottky contact on n-type Si with a conducting polymer," Journal of Physics D: Applied Physics, vol. 42, p. 165104, 2009.

[138] Heraeus, "Clevios PH1000," 2016.

[139] J. Maeng, M. Jo, S. Kang, M. Kwon, G. Jo, T. Kim, J. Seo, H. Hwang, D. Kim, S. Park, and T. Lee, "Transient reverse current phenomenon in a p-n heterojunction comprised of poly(3,4-ethylene-dioxythiophene):poly(styrene-sulfonate) and ZnO nanowall," Applied Physics Letters, vol. 93, p. 123109, 2008.

[140] D. L. Meier, J. Hwang, and R. B. Campbell, "The Effect of Doping Density and Injection Level on Minority-Carrier Lifetime as Applied to Bifacial Dendritic Web Silicon Solar Cells," IEEE Transactions on Electron Devices, vol. ED-35, pp. 70-79, 1988. 
[141] E. Fabre, M. Mautref, and A. Mircea, "Trap saturation in silicon solar cells," Applied Physics Letters, vol. 27, pp. 239-240, 1975.

[142] P. C. Mathur, J. D. Arora, R. P. Sharma, and P. Saxena, "Dependence of minority carrier diffusion length on illumination level and temperature in single crystal and polycrystalline Si solar cells," Journal of Applied Physics, vol. 52, pp. 6949-6953, 1981.

[143] C. T. Ho, R. O. Bell, and F. V. Wald, "Enhancement of diffusion length in EFG ribbon solar cells under illumination," Applied Physics Letters, vol. 31, pp. 463-465, 1977.

[144] N. D. Arora, J. R. Hauser, and D. J. Roulston, "Electron and Hole Mobilities in Silicon as a Function of Concentration and Temperature," IEEE Transactions on Electron Devices, vol. 29, pp. 292-295, 1982.

[145] S. Suckow, T. M. Pletzer, and H. Kurz, "Fast and reliable calculation of the two-diode model without simplifications," Prog. Photovolt: Res. Appl., 22: 494-501, vol. 22, pp. 494501, 2014.

[146] M. Wolf, G. T. Noel, and R. J. Stirn, "Investigation of the double exponential in the current-voltage characteristics of silicon solar cells," IEEE Transactions on Electron Devices, vol. 24, pp. 419-428, 1977.

[147] Y. Chang, K. Lee, R. Kiebooms, A. Aleshin, and A. J. Heeger, "Reflectance of conducting poly(3,4-ethylenedioxythiophene)," Synthetic Metals, vol. 105, pp. 203-206, 15 September 19991999.

[148] T. Stöcker, A. Köhler, and R. Moos, "Why does the electrical conductivity in PEDOT:PSS decrease with PSS content? A study combining thermoelectric measurements with impedance spectroscopy," Journal of Polymer Science Part B: Polymer Physics, vol. 50, pp. 976-983, 2012.

[149] M. J. Sailor, F. L. Klavetter, R. H. Grubbs, and N. S. Lewis, "Electronic properties of junctions between silicon and organic conducting polymers," Nature, vol. 346, pp. 155157, 07/12/print 1990.

[150] H. M. Zeyada and M. M. Makhlouf, "Electrical conduction mechanisms and dielectric constants of nanostructured methyl violet 2B thin film," Appl. Phys. A, vol. 119, pp. 1109-1118, 2015.

[151] H. M. Zeyada, A. A. Habashi, M. M. Makhlouf, A. S. Behairy, and M. A. Nasher, "Fabrication, electrical transport mechanisms and photovoltaic properties of methyl violet 2B/n-Si hybrid organic/inorganic solar cell," Microelectronic Engineering, vol. 163, pp. 134-139, 2016.

[152] Silvaco. (2013). ATLAS User's Manual.

[153] F. Zhang, T. Song, and B. Sun, "Conjugated polymer-silicon nanowire array hybrid Schottky diode for solar cell application," Nanotechnology, vol. 23, p. 194006, 2012.

[154] T.-G. Chen, B.-Y. Huang, E.-C. Chen, P. Yu, and H.-F. Meng, "Micro-textured conductive polymer/silicon heterojunction photovoltaic devices with high efficiency," Applied Physics Letters, vol. 101, p. 033301, 2012.

[155] Q. Pei, G. Zuccarello, M. Ahlskog, and O. Inganäs, "Electrochromic and highly stable poly(3,4-ethylenedioxythiophene) switches between opaque blue-black and transparent sky blue," Polymer, vol. 35, pp. 1347-1351, 1994.

[156] M. Dietrich, J. Heinze, G. Heywang, and F. Jonas, "Electrochemical and spectroscopic characterization of polyalkylenedioxythiophenes," Journal of Electroanalytical Chemistry, vol. 369, pp. 87-92, 1994.

[157] S. Fonash, Solar Cell Device Physics, 2010.

[158] W. Brütting, Introduction to the Physics of Organic Semiconductors. Institute of Physics, University of Augsburg, Germany: John Wiley and Sons, 2006. 
[159] J. G. Fossum and D. S. Lee, "A physical model for the dependence of carrier lifetime on doping density in nondegenerate silicon," Solid-State Electronics, vol. 25, pp. 741-747, 1982.

[160] M. E. Law, E. Solley, M. Liang, and D. E. Burk, "Self-consistent model of minority-carrier lifetime, diffusion length, and mobility," IEEE Electron Device Letters, vol. 12, pp. 401403, 1991.

[161] D. J. Roulston, N. D. Arora, and S. G. Chamberlain, "Modeling and Measurement of Minority-Carrier Lifetime versus Doping in Diffused Layers of $\mathrm{n} \pm \mathrm{p}$ Silicon Diodes," IEEE Trans. Electron Devices, vol. 29, pp. 284-291, 1982.

[162] J. Dziewior and W. Schmid, "Auger coefficients for highly doped and highly excited silicon," Applied Physics Letters, vol. 31, pp. 346-348, 1977.

[163] D. M. Caughey and R. E. Thomas, "Carrier Mobilities in Silicon Empirically Related to Doping and Field," Proc. IEEE 55, pp. 2192-2193, 1967.

[164] D. Diouf, J. P. Kleider, T. Desrues, and P. J. Ribeyron, "Study of interdigitated back contact silicon heterojunctions solar cells by two-dimensional numerical simulations," Materials Science and Engineering: B, vol. 159-160, pp. 291-294, 2009.

[165] X. Loozen, J. B. Larsen, F. Dross, M. Aleman, T. Bearda, B. J. O'Sullivan, I. Gordon, and J. Poortmans, "Passivation of a Metal Contact with a Tunneling Layer," Energy Procedia, vol. 21, pp. 75-83, 2012.

[166] M. J. Kerr and A. Cuevas, "Recombination at the interface between silicon and stoichiometric plasma silicon nitride," Semicond. Sci. Technol., vol. 17, pp. 166-172, 2002.

[167] Y. H. Kim, C. Sachse, M. L. Machala, C. May, L. Müller-Meskamp, and K. Leo, "Highly conductive PEDOT:PSS electrode with optimized solvent and thermal post-treatment for ITO-free organic solar cells," Advanced Functional Materials, vol. 21, pp. 1076-1081, 2011.

[168] B. Charlot, G. Sassine, A. Garraud, B. Sorli, A. Giani, and P. Combette, "Micropatterning PEDOT:PSS layers," Microsystem Technologies, vol. 19, pp. 895-903, 2013.

[169] J. Szlufcik, S. Sivoththaman, J. F. Nlis, R. P. Mertens, and R. Van Overstraeten, "Low-cost industrial technologies of crystalline silicon solar cells," in Proceedings of the IEEE, 1997, pp. 711-730.

[170] T. Dullweber, S. Gatz, H. Hannebauer, T. Falcon, R. Hesse, J. Schmidt, and R. Brendel, "Towards 20\% efficient large-area screen-printed rear-passivated silicon solar cells," Progress in Photovoltaics: Research and Applications, vol. 20, pp. 630-638, 2012.

[171] M. A. Green, Solar Cells - Operating Principles, Technology and System Application. Kensington, NSW 2033: The University of New South Wales, 1998.

[172] T. Saga, "Advances in crystalline silicon solar cell technology for industrial mass production," NPG Asia Materials, vol. 2, pp. 96-102, Jul 2010.

[173] Y. Zhang, F. Zu, S.-T. Lee, L. Liao, N. Zhao, and B. Sun, "Heterojunction with Organic Thin Layers on Silicon for Record Efficiency Hybrid Solar Cells," Advanced Energy Materials, vol. 4, pp. n/a-n/a, 2014.

[174] I. Irfan, A. James Turinske, Z. Bao, and Y. Gao, "Work function recovery of air exposed molybdenum oxide thin films," Applied Physics Letters, vol. 101, p. 093305, 2012.

[175] I. Irfan, H. Ding, Y. Gao, C. Small, D. Y. Kim, J. Subbiah, and F. So, "Energy level evolution of air and oxygen exposed molybdenum trioxide films," Applied Physics Letters, vol. 96, p. 243307, 2010.

[176] T. M. Brown, J. S. Kim, R. H. Friend, F. Cacialli, R. Daik, and W. J. Feast, "Built-in field electroabsorption spectroscopy of polymer light-emitting diodes incorporating a doped 
poly(3,4-ethylene dioxythiophene) hole injection layer," Applied Physics Letters, vol. 75, pp. 1679-1681, 1999.

[177] S. K. So, W. K. Choi, C. H. Cheng, L. M. Leung, and C. F. Kwong, "Surface preparation and characterization of indium tin oxide substrates for organic electroluminescent devices," Appl. Phys. A, vol. 68, pp. 447-450, 1999.

[178] S. Y. Kim, J.-L. Lee, K.-B. Kim, and Y.-H. Tak, "Effect of ultraviolet-ozone treatment of indium-tin-oxide on electrical properties of organic light emitting diodes," Journal of Applied Physics, vol. 95, pp. 2560-2563, 2004.

[179] C. Wang, I. Irfan, and Y. Gao, "Protection of $\mathrm{MoO} 3$ high work function by organic thin film," Applied Physics Letters, vol. 105, p. 181602, 2014.

[180] J. Sheng, K. Fan, D. Wang, C. Han, J. Fang, P. Gao, and J. Ye, "Improvement of the SiO Passivation Layer for High-Efficiency Si/PEDOT:PSS Heterojunction Solar Cells," ACS Appl Mater Interfaces, 2014.

[181] F. I. Simjee and P. H. Chou, "Efficient Charging of Supercapacitors for Extended Lifetime of Wireless Sensor Nodes," IEEE Transactions on Power Electronics, vol. 23, pp. 15261536, 2008.

[182] F. Ongaro, S. Saggini, and P. Mattavelli, "Li-lon Battery-Supercapacitor Hybrid Storage System for a Long Lifetime, Photovoltaic-Based Wireless Sensor Network," IEEE Transactions on Power Electronics, vol. 27, pp. 3944-3952, 2012.

[183] D. Brunelli, L. Benini, C. Moser, and L. Thiele, "An Efficient Solar Energy Harvester for Wireless Sensor Nodes," presented at the 2008 Design, Automation and Test in Europe, Munich 2008.

[184] S. Escolar, S. Chessa, and J. Carretero, "Energy management in solar cells powered wireless sensor networks for quality of service optimization," Personal and Ubiquitous Computing, vol. 18, pp. 449-464, 2013.

[185] G. Chen, H. Ghaed, R.-u. Haque, M. Wieckowski, Y. Kim, G. Kim, D. Fick, D. Kim, M. Seok, K. Wise, D. Blaauw, and D. Sylvester, "A Cubic-Millimeter Energy-Autonomous Wireless Intraocular Pressure Monitor," presented at the 2011 IEEE International Solid-State Circuits Conference, 2011.

[186] A. Bozzola, P. Kowalczewski, and L. C. Andreani, "Towards high efficiency thin-film crystalline silicon solar cells: The roles of light trapping and non-radiative recombinations," Journal of Applied Physics, vol. 115, p. 094501, 2014.

[187] K. E. Petersen, "Silicon as a Mechanical Material," Proceedings of the IEEE, vol. 70, pp. 420-457, 1982.

[188] M. A. Hopcroft, W. D. Nix, and T. W. Kenny, "What is the Young's Modulus of Silicon?," Journal of Microelectromechanical Systems, vol. 19, pp. 229-238, 2010.

[189] E. J. Boyd and D. Uttamchandani, "Measurement of the Anisotropy of Young's Modulus in Single-Crystal Silicon," Journal of Microelectromechanical Systems, vol. 21, pp. 243249, 2012.

[190] S. J. Cox and C. M. McCarthy, "The Shape of the Tallest Column," Society for Industrial and Applied Mathematics, vol. 29, pp. 547-554, 1998.

[191] K. H. Kim, K. C. Park, and D. Y. Ma, "Structural, electrical and optical properties of aluminum doped zinc oxide films prepared by radio frequency magnetron sputtering," Journal of Applied Physics, vol. 81, p. 7764, 1997.

[192] J. Wang, H. Wang, A. B. Prakoso, A. S. Togonal, L. Hong, C. Jiang, and Rusli, "High Efficiency Silicon Nanowire/Organic Hybrid Solar Cells with Two-Step Surface Treatment," Nanoscale, vol. 7, pp. 4559-65, 2015. 
[193] S. Ouyang, Y. Xie, D. Zhu, X. Xu, D. Wang, T. Tan, and H. H. Fong, "Photolithographic patterning of PEDOT:PSS with a silver interlayer and its application in organic light emitting diodes," Organic Electronics, vol. 15, pp. 1822-1827, 2014. 